\title{
Challenging the serotonin system : a mechanistic approach to the method of acute tryptophan depletion in rodents
}

Citation for published version (APA):

van Donkelaar, E. L. (2009). Challenging the serotonin system : a mechanistic approach to the method of acute tryptophan depletion in rodents. [Doctoral Thesis, Maastricht University]. Datawyse / Universitaire Pers Maastricht. https://doi.org/10.26481/dis.20090917ed

Document status and date:

Published: 01/01/2009

DOI:

10.26481/dis.20090917ed

Document Version:

Publisher's PDF, also known as Version of record

Please check the document version of this publication:

- A submitted manuscript is the version of the article upon submission and before peer-review. There can be important differences between the submitted version and the official published version of record.

People interested in the research are advised to contact the author for the final version of the publication, or visit the DOI to the publisher's website.

- The final author version and the galley proof are versions of the publication after peer review.

- The final published version features the final layout of the paper including the volume, issue and page numbers.

Link to publication

\footnotetext{
General rights rights.

- You may freely distribute the URL identifying the publication in the public portal. please follow below link for the End User Agreement:

www.umlib.nl/taverne-license

Take down policy

If you believe that this document breaches copyright please contact us at:

repository@maastrichtuniversity.nl

providing details and we will investigate your claim.
}

Copyright and moral rights for the publications made accessible in the public portal are retained by the authors and/or other copyright owners and it is a condition of accessing publications that users recognise and abide by the legal requirements associated with these

- Users may download and print one copy of any publication from the public portal for the purpose of private study or research.

- You may not further distribute the material or use it for any profit-making activity or commercial gain

If the publication is distributed under the terms of Article $25 \mathrm{fa}$ of the Dutch Copyright Act, indicated by the "Taverne" license above, 


\section{Challenging the serotonin system}

A mechanistic approach to the method of acute tryptophan depletion in rodents

Eva L. van Donkelaar 
Copyright (C) Eva L. van Donkelaar, Maastricht 2009 ISBN: 978-90-9024542-3

Challenging the serotonin system: A mechanistic approach to the method of acute tryptophan depletion in rodents

Thesis with summary in English, Dutch and Spanish

All rights reserved. No part may be reproduced or transmitted in any form or by any means, electronic or mechanical including photocopying, recording or by any information storage and retrieval system, without permission, in writing, from the copyright holder.

Coverdesign: Jaime W. de Meij \& Dani Caballero

Typesetting and layout: Eva L. van Donkelaar \& Borja Roses Cañete

Production: Datawyse, University Press Maastricht 


\section{Challenging the serotonin system}

A mechanistic approach to the method of acute tryptophan depletion in rodents

\section{Proefschrift}

ter verkrijging van de graad van doctor aan de Universiteit Maastricht, op gezag van de Rector Magnificus,

Prof. mr. G.P.M.F. Mols, volgens het besluit van het college van Decanen, in het openbaar te verdedigen op donderdag 17 september 2009 om 14.00 uur door

\section{Eva Luisa van Donkelaar}

Geboren op 5 juli 1978 te Jerez de la Frontera 


\section{Promotor}

Prof. dr. H.W.M. Steinbusch

\section{Copromotores}

Dr. J. Prickaerts

Dr. P.A.T. Kelly (University of Edinburgh, United Kingdom)

Dr. A. Blokland

\section{Review Committee}

Prof. dr. E.J.L. Griez (chairman)

Dr. J. Bakker (Université de Liège, Belgium)

Prof. dr. B.E. Leonard

Dr. J.L. Pawluski

Prof. dr. W.J. Riedel

All studies described in this thesis were funded by the European Commission Research Directorates, Research \& Technological Development Project, 6 ${ }^{\text {th }}$ Framework Programme (NEWMOOD). Contract grant number: LSHM-CT-2003-503474.

The publication of this thesis was financially supported by Maastricht University, School for Mental Health and Neuroscience (MHeNS) and a EUROGENDIS award to the University of Edinburgh. 


\section{Por mi Roses}

Universidad de la Vida 



\section{Contents}

Chapter 1

Chapter 2

Acute tryptophan depletion in healthy C57BL/6 mice does not induce reduction of central serotonin levels or affective behavioural changes

\section{Chapter 3}

Acute tryptophan depletion in rats alters the relationship between cerebral blood flow and glucose metabolism independent of central serotonin

Chapter 4 105

Altered cerebrovascular control following acute tryptophan depletion in MDMA-pretreated rats

Chapter 5

Stress-mediated decreases in brain-derived neurotrophic factor as potential confounding factor for acute tryptophan depletion-induced neurochemical effects

Chapter 6 155

Phosphodiesterase 2 and 5 inhibition attenuates the object memory deficit induced by acute tryptophan depletion

\section{Chapter 7}

General Discussion

$\begin{array}{rr}\text { Summary } & 197 \\ \text { Samenvatting } & 201 \\ \text { Resumen } & 207 \\ \text { Acknowledgements } & 213 \\ \text { Curriculum Vitae } & 217 \\ \text { Publications } & 219\end{array}$





\section{1}

\section{General Introduction}

Eva L. van Donkelaar 

The monoamine hypothesis of depression was born in the 1950 s with the accidental discovery of the antidepressant effects of compounds initially used to treat other disease states. Both iproniazid, a monoamine oxidase inhibitor prescribed for the treatment of tuberculosis, and imipramine, a tricyclic compound initially used as antipsychotic, appeared to effectively relieve depressive symptoms by boosting the levels of the neurotransmitters serotonin (5-hydroxytryptamine; 5-HT), noradrenaline (NE) and to a lesser extent dopamine (DA; Crane, 1957; Kuhn, 1958). Moreover, the antihypertensive reserpine was shown to considerably deplete monoamine stores which was thought to be responsible for its apparent depressive-like effects (Pletscher et al., 1955; Shore et al., 1955). Based on these early pharmacological observations of chemical imbalances, depressive illness was thought to be caused by a deficiency in these monoamine levels (France et al., 2007; Schatzberg et al., 2002). Consequently, experimental monoamine depletion methods have been extensively applied for a better understanding of the implication of these neurotransmitter systems in the aetiology and treatment of mood disorders.

Acute tryptophan depletion (ATD) currently represents the most established human challenge test to investigate the involvement of the 5-HT system in the pathogenesis and pathophysiology of depression. Since the method does not imply the use of neurotoxins it can be used to repeatedly manipulate the central 5-HT system in vivo and assess the behavioural effects of reduced 5-HT metabolism in the brain. However, the exact mechanism by which ATD exerts its neurophysiological effects and to what extent changes in 5-HT neuronal activity contribute to ATD-induced neurochemical and behavioural alterations is not clear. This impedes an adequate interpretation of the results arising from application of the method in both clinical and preclinical studies. Yet, manipulation of tryptophan (TRP) concentrations in the diet is not only a straightforward tool for effectively decreasing brain TRP content, it also provides a clue to understanding the mechanism behind the regulation of the 5 -HT system in general.

\section{Tryptophan, Serotonin and Depression}

\section{From essential amino acid to modulatory neurotransmitter}

Amino acids are molecules that play a central role in biochemistry as they form the basic structural building units of proteins and serve as precursors for biosynthesis of other molecules. Due to their pivotal role in metabolism they are critical to life and, thus, highly important in nutrition. Of the 20 standard amino acids that are used for protein synthesis (Table 1), 12 non-essential amino acids can be produced by adults in sufficient quantities out of other chemicals (Young, 1994). Tryptophan (TRP), together with phenylalanine (PHE), valine (VAL), threonine, (THR) isoleucine (ILE), methionine (MET), 
leucine (LEU) and lysine (LYS) cannot be synthesized de novo by the body itself. Therefore, in order to obtain TRP, its inclusion in the diet is essential (Rose et al., 1954) and this is why it is referred to as an essential amino acid. This implies that the amount of "dietary 5-HT precursor" eventually available to the organism can easily be manipulated, a major point on which the method of ATD is based.

Table 1 Amino acids necessary for protein synthesis, classified into essential and non-essential according to the ability of de novo biosynthesis by the adult body. (*) large neutral amino acids competing at the blood-brain barrier for transport into the brain

\begin{tabular}{ll}
\hline Essential & Non-essential \\
\hline Isoleucine* & Alanine \\
Leucine* & Arginine \\
Lysine & Asparagine \\
Methionine & Aspartic Acid \\
Phenylalanine* & Citrulline \\
Threonine & Cysteine \\
Tryptophan* & Glutamic Acid \\
Valine* & Glycine \\
& Histidine \\
& Proline \\
& Serine \\
\hline
\end{tabular}

By decreasing the availability of TRP in plasma, ATD ultimately aims to diminish the amount of 5-HT neurotransmitter in the brain. Yet, the underlying assumptions for applying the method as a serotonergic challenge tool eventually arise from the specific characteristics of the biosynthesis from TRP to 5-HT. Not only is the rate of 5-HT synthesis controlled by the amount of TRP available in 5-HT producing neurons, TRP initially needs to compete with other amino acids for transport across the blood-brain barrier and is the only amino acid that binds to blood albumin in the periphery.

\section{From tryptophan to serotonin}

TRP taken up into 5-HT producing neurons is synthesized into 5-HT by a two step reaction (Figure 1) involving the hydroxylation of TRP into the naturally occurring amino acid 5-hydroxytryptophan (5-HTP) followed by the decarboxylation of 5-HTP into 5-HT (Boadle-Biber, 1993). The hydroxylation of TRP into 5-HTP is catalyzed by the enzyme tryptophan hydroxylase (TPH), however, the Michaelis constant $\left(\mathrm{K}_{\mathrm{m}}\right)$ of TPH for TRP is much higher than the amount of TRP normally present in the brain. This implies that, under normal 
physiological conditions (in vivo), the enzyme is not fully saturated and the activity of TPH is so-called substrate-driven. In other words, the rate at which TRP is hydroxylated by TPH into 5-HTP is limited by substrate concentration and therefore considered as the rate-limiting step within the 5-HT biosynthetic pathway.

After hydroxylation into 5-HTP, decarboxylation by the enzyme aromatic L-amino acid decarboxylase (AAAD) finalizes the synthesis of 5-HTP into 5-HT. The principal metabolite of 5-HT, 5-hydroxyindoleacetic acid (5-HIAA), is inactive and formed by oxidative deamination of 5-HT to an aldehyde intermediate catalyzed by monoamine oxidase (MAO) with subsequent oxidation by aldehyde dehydrogenase (ADH) into 5-HIAA (Boadle-Biber, 1993). Thus, a decrease in brain TRP levels affects the degree of TPH saturation and thereby the amount of TRP converted into 5-HTP and subsequent 5-HT formation. This assumption has initially served for using ATD as a method to systematically decrease 5-HT synthesis, which is likely to result in decreased levels of the inactive metabolite 5-HIAA. However, the amount of TRP inside 5-HT producing neurons initially depends upon the degree of TRP in plasma that gets into the brain (Fernstrom and Wurtman, 1971; Fernstrom, 1983). Therefore, 5-HT synthesis is eventually controlled by the amount of its dietary precursor TRP available for transport through the blood-brain barrier (BBB). Consequently, dietary manipulation of the essential amino acid TRP by the method of ATD can interfere with normal brain 5-HT synthesis and metabolism.
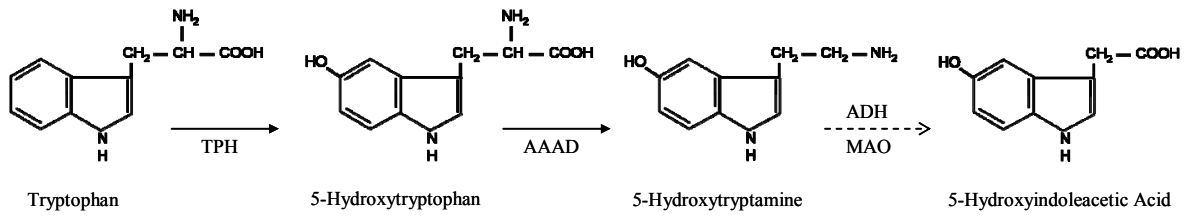

Figure 1 Central serotonin synthesis and metabolism

In the brain, tryptophan (TRP) is first hydroxylated into 5-hydroxytryptophan (5-HTP) by the enzyme tryptophan hydroxylase (TPH). Aromatic L-amino acid decarboxylase (AAAD) subsequently catalyzes the decarboxylation of 5-HTP into 5-hydroxytryptamine (5-HT). The enzymes monoamine oxidase (MAO) and aldehyde dehydrogenase (ADH) eventually break serotonin (5-hydroxytryptamine, 5-HT) down into the inactive metabolite 5-hydroxyindoleacetic acid (5-HIAA).

\section{From plasma to brain tryptophan}

The only way amino acids can be transported from the blood through the capillary endothelial cells of the $\mathrm{BBB}$ into the brain is by carrier-mediated transporter systems in the capillary cell plasma membranes (Oldendorf, 1971). Given that the surface area of the $\mathrm{BBB}$ is much smaller compared to the surface area of brain cell membranes, it is this initial transport through the BBB 
that limits the uptake of plasma TRP into the brain (Pardridge, 1998). The brand-chain amino acids (BCAAs; LEU, ILE, VAL) together with the aromatic amino acids PHE, tyrosine (TYR) and TRP, are sub-classified as large neutral amino acids (LNAAs) according to the specificity of their "large, neutral" side chains and subsequent physiological function. Of the nine different amino acid transport systems identified at the BBB, the so-called Transport System L is only half saturated under normal physiological conditions and mediates high affinity, sodium-independent uptake of all LNAAs (Pardridge, 1998; Smith, 2000). Consequently, for binding to the L-amino acid transport carrier and subsequent transport into the brain, TRP has to compete heavily with the other LNAAs (Fernstrom and Wurtman, 1971, 1972b; Gessa et al., 1974). This implies that the amount of TRP available to 5-HT producing neurons additionally depends upon the amount of other LNAAs in plasma. The availability of TRP in the brain thus depends upon the ratio of TRP to the sum of the other LNAAs (TRP/ $\Sigma$ LNAA) and a decrease in this ratio in plasma is normally used as the best predictor of reduced availability of TRP in the brain and subsequent synthesis into 5-HT (Fernstrom, 1979b, 1983).

\section{From bound to free plasma tryptophan; the brain influx parameter}

About $90 \%$ of all TRP molecules circulating in blood are bound to serum albumin. However, both endogenous ligands, such as non-esterified fatty acids (NEFAs; Curzon et al., 1973; Curzon and Knott, 1973), and exogenous ligands, such as acetylsalicylic acid (Smith and Lakatos, 1971), can displace TRP from its protein binding. Although positive correlations between serum free-TRP and whole brain TRP levels have been reported in rats (Biggio et al., 1974; Oldendorf and Szabo, 1976), dissociation of TRP from albumin by endogenous and exogenous ligands has been shown to increase the entry of TRP into the brain, thereby enhancing central 5-HT synthesis (Gessa and Tagliamonte, 1974; Tagliamonte et al., 1973). The ratio between TRP-free and TRP-bound levels can therefore vary depending on the presence of other circulating substances that displace TRP from albumin. This observation, together with the fact that TRP is the only amino acid that is bound to serum proteins suggests that only free TRP is available for transport into the brain (Biggio et al., 1974). Moreover, the changes in TRP-free levels can take place independently of changes in total TRP levels (Bender, 1983), which would make a distinction between free and bound TRP necessary for estimating its availability in the brain.

The free TRP fraction appears to be a very sensitive parameter, not only easily influenced by pharmacological modulation, but also by physiological aspects such as food intake or deprivation, hormones, exercise and mild stressors (Badawy, 2009). Moreover, methodological differences in separating and determining free and albumin-bound TRP fractions in blood samples interferes considerably with proper interpretation of behavioural and biochemical results (Fernstrom, 1979a). Although the fraction of free TRP 
seems a valuable tool to estimate the amount of TRP in the circulation available for uptake into various tissues and organs (Badawy, 2009), accumulating evidence supports the fact that total peripheral TRP concentrations (free plus bound) better reflect the rate of influx of TRP into the brain. It has been shown that TRP is only loosely bound to albumin and although albumin itself cannot cross the $\mathrm{BBB}$, it appears to be a highly flexible protein undergoing reversible conformational changes (Reed and Burrington, 1989). These conformational changes, which occur during transport of TRP from the circulating albumin-bound pool, enhance the dissociation of TRP from the albumin binding sites within the cerebral microvasculature and appear to be highly dependent upon cerebral haemodynamics (Pardridge and Fierer, 1990). Low cerebral blood flow is likely to increase the interaction between the albumin-bound TRP complex and the glycocalyx of the BBB, thereby causing more TRP to dissociate from albumin (Pardridge and Fierer, 1990; Smith et al., 1987). This implies that temporally dynamic or spatial differences in local cerebral blood flow may influence the rate of central TRP uptake in general and even within specific brain areas only (Ruddick et al., 2006). Thus, although only free TRP can eventually cross the BBB, the amount of albumin-bound TRP in plasma must also be taken into account to calculate the availability of TRP in the brain, as TRP can easily dissociate from albumin near the BBB thereby increasing the TRP-free pool and subsequent uptake into the brain (see also Figure 2).

\section{From tryptophan to kynurenine}

Under normal physiological conditions, only 1-2\% of the amount of ingested TRP is used by the body for the synthesis of 5-HT (Twarog and Page, 1953). The majority of total ingested TRP is catabolyzed into kynurenine (KYN) by induction of tryptophan pyrrolase in the liver (Stone and Darlington, 2002). Tryptophan pyrrolase is the first rate-limiting enzyme of the kynurenine pathway and KYN is the major degradation product of TRP (Moroni, 1999).

Induction of pyrrolase by the enzymes indolamine 2,3-dioxygenase (IDO) and tryptophan 2,3-dioxygenase (TDO) in the liver reduces TRP availability (Botting, 1995) and therefore 5-HT synthesis is also influenced by IDO and TDO activity (Moroni, 1999; Smith and Pogson, 1980). Stimulation of these enzymes by proinflammatory cytokines, in particular interferon- $\gamma$ ( $\mathrm{Hu}$ et al., 1995), enhances the catabolism of TRP (Botting, 1995), thereby decreasing the amount of TRP eventually available for 5-HT synthesis. Moreover, TDO activity can also be induced by corticoids (Turner et al., 2006). 


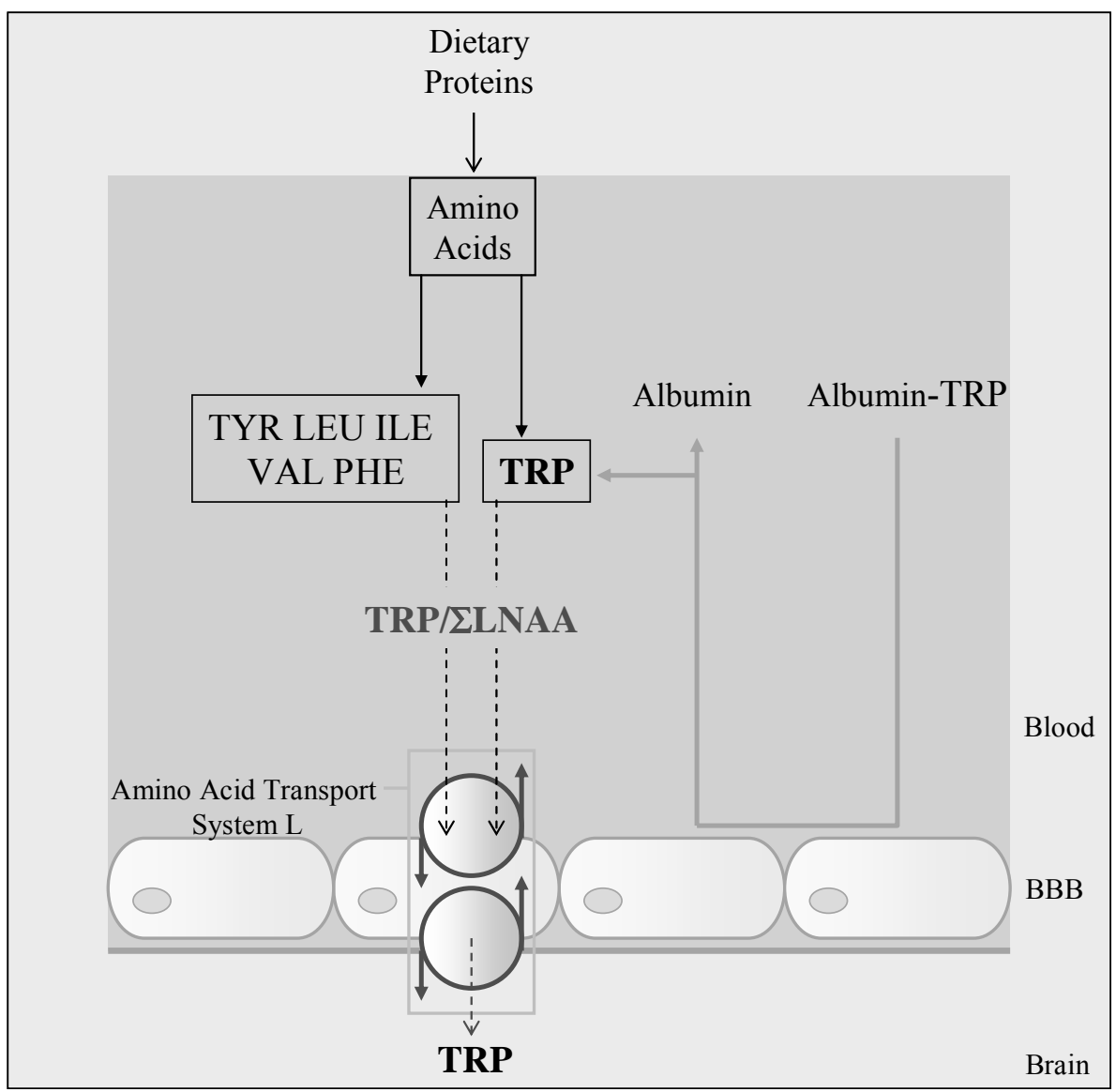

Figure 2 Overview of tryptophan metabolism from food intake to brain uptake

In order to obtain the amino acid tryptophan (TRP) its inclusion in the diet is essential. The majority of TRP is bound to plasma albumin and only free TRP will eventually cross the bloodbrain barrier (BBB). Albumin-bound TRP easily dissociates from albumin near the cerebrovasculature under the influence of haemodynamic changes, thereby increasing the TRPfree fraction available for uptake into the brain. The amount of TRP in plasma eventually crossing the BBB also depends upon the presence of other large neutral amino acids (LNAAs) that all compete for the same amino acid transport system L at the BBB. Due to this competition of TRP with leucine (LEU), isoleucine (ILE), valine (VAL), phenylalaline (PHE) and tyrosine (TYR), the ratio of TRP to the sum of these LNAAs (TRP/ $L$ LNAA) in plasma better reflects the amount of central TRP available for synthesis into 5-HT.

\section{The modulatory serotonin neurotransmitter}

Although "enteramine" was first discovered by Erspamer in the early 1930s as a substance with smooth muscle contracting properties in the enterochromaffin cells of the gut (Erspamer and Vialli, 1937; Whitaker-Azmitia, 1999), it was not until 1948 that Rapport et al. (1948a) first isolated a factor with "tonic" or vasocontrictive actions in serum released from platelets following blood 
clotting. They first referred to their "serum tonic factor" as serotonin (Page et al., 1948; Rapport et al., 1948b) and subsequently identified it as 5-hydroxytryptamine (Page, 1954) which later appeared to be the same substance as Erspamer's "enteramine". In 1953, the same group first detected "serotonin" in brain tissue (Page, 1976) which, together with the gut and blood serum, completed the key localisations of what later appeared to be a multifunctional neurotransmitter (see also: Sjoerdsma and Palfreyman, 1990).

Although $90 \%$ of all 5 -HT is localized in the gastrointestinal tract and the amount of 5-HT neurons in the brain is relatively small compared to the total numbers of neurons, many neurophysiological processes are known to be regulated by the 5 -HT system, including mood and cognition, which are most prominently impaired in clinical depression (Leonard, 1997; Maes and Meltzer, 1995; Meltzer, 1990). By means of immunohistochemical methods with a highly specific polyclonal antiserum directed against 5-HT it has been possible to precisely map the 5-HT system in detail (Steinbusch et al., 1978; Steinbusch, 1981). 5-HT cell bodies are clustered in the brainstem raphe nuclei sending out projections throughout the entire central nervous system with ascending pathways innervating anatomically and functionally diverse regions of the cerebral cortex, including the limbic system, the basal ganglia and structures within the diencephalon (Steinbusch, 1981). Due to this anatomy, the neurotransmitter influences all regions of the neuraxis, thereby modulating an extensive range of physiological and behavioural functions. Besides mood and cognition, appetite, emesis, endocrine function, gastrointestinal function, motor function, neurotrophism, perception, sensory function, pain sensitivity, sex, sleep and even vascular function are all under the control of the 5-HT system. Consequently, disrupted 5-HT synthesis and subsequent abnormal 5-HT function can lead to a diverse range of behavioural disturbances implicated in clinical depression (Table 2).

Table 2 Clinically relevant behaviours modulated by 5-HT and implicated in major depressive disorder when disturbed.

\begin{tabular}{ll}
\hline Mood regulation & Sexual function \\
Fear and anxiety & Motivation an reward \\
Learning and memory & Neuroendocrine regulation \\
Cognitive control & Circadian rhythm regulation \\
Appetite and eating regulation & Stress response \\
Sleep & Impulse control \\
\hline
\end{tabular}

\section{5-HT and depression}

Clinical depression is the most prevalent mental illness, symptom clustered under mood disorders and specifically classified as major depressive disorder (MDD) in the Diagnostic and Statistical Manual of Mental Disorders Text 
Revision (DSM-IV-TR, 2000) of the American Psychiatric Association. It is generally characterized by sadness, loss of interest or pleasure, feelings of guilt or low self-esteem, disturbed sleep or appetite, low energy and poor concentration. Sustained sadness along with psychological, behavioural and physical symptoms can become chronic or recurrent, eventually impairing the ability to cope with daily life. Worldwide, depression is one of the leading causes of illness-induced disability and at its most severe can lead to suicide, which is among the three leading causes of death among those aged 15-44 years (Murray and Lopez, 1997). Yet, the underlying genetic and neurobiological mechanisms of depressive illness and the controversial responses to different treatments are still not fully understood (Nestler et al., 2002).

Major depression is a heterogeneous disorder displaying a high prevalence of co-morbidity with other neuropathological syndromes such as generalized anxiety. Nowadays, besides dysfunctional monoamine systems, disturbances in endocrine and immune function and adaptive changes in pre- and postsynaptic receptors have taken a prominent position in theories concerning the aetiology and pathogenesis of depressive symptoms (Hindmarch, 2002; Leonard, 2007). Nevertheless, the pathophysiology of most types of depression, as well as genetic vulnerability factors that increase the risk for suffering depressive episodes, involve abnormal functioning of the 5-HT system (Meltzer, 1990). Therefore, 5-HT dysfunction is widely accepted as one of the main causes underlying the development of depressive symptomatology (Asberg et al., 1976a; Maes and Meltzer, 1995; Meltzer, 1990). Direct evidence supporting the central role of 5-HT in depression comes from human studies correlating depressive symptoms to decreased physiological parameters, indicative of alterations in the 5-HT system (Risch and Nemeroff, 1992). Consistent findings specifically include decreased peripheral TRP levels (Cowen et al., 1989) and lower levels of the inactive 5-HT metabolite 5-HIAA in cerebral spinal fluid (CSF), which all reflect diminished 5-HT metabolism (Asberg et al., 1976b; van Praag and de Haan, 1979). The effectiveness of serotonergic drugs used in the treatment of depression also confirms the important role of disrupted function of specific pre- and postsynaptic receptors underlying impaired 5-HT neurotransmission and linked to specific depressive symptoms (Cryan and Leonard, 2000; Naughton et al., 2000).

\section{Acute tryptophan depletion and depression research}

\section{The method of acute tryptophan depletion}

Whereas ATD is based upon decreasing 5-HT synthesis by lowering the metabolism of its precursor, other interventions can be used to more directly interrupt central 5-HT systems. However, animal studies showed that direct manipulations by means of central injections with 5,7-dihydroxytryptamine 
(5,7-DHT) or peripheral application of TPH inhibitors (parachlorophenylalanine: PCPA; p-ethynylphenylalanine: $\mathrm{p}-\mathrm{EDH}$ ), but also acute and repeated treatment with amphetamine derivates such as 3,4-methylenedioxymethamphetamine (MDMA; 'ecstasy') or 5-HT receptor ligands, cause serious side-effects and tend to be neurotoxic, thereby causing irreversible damage with long-term behavioural consequences. Moreover, these lesions and pharmacological manipulations are not highly specific and normally induce almost complete depletion of central 5-HT. This, together with the induced neurotoxicity, seems less appropriate for studying behavioural changes linked to a dysfunctional 5-HT system, which is characterized by reduced, i.e. not completely absent, 5-HT synthesis (Reilly et al., 1997). ATD, on the other hand, is non-toxic and nonintrusive, thereby providing the option to repeatedly manipulate the central 5-HT system in vivo and assess the behavioural effects of reduced 5-HT metabolism in the brain (Fadda et al., 2000). The reduction of brain 5-HT in a reversible manner reflects the main methodological advantage of the tool, permitting application of the same basic method in both human subjects and rodents. This is considered valuable for comparing neurophysiological changes linked to behavioural effects across species (Blokland et al., 2004).

A rather slow reduction in peripheral and central TRP levels and a concomitant decrease of brain 5-HT concentrations was observed after longterm administration of diets low in TRP or completely lacking the essential amino acid. However, severe malnutrition, especially in the latter case, interferes with appropriate interpretation of the specific behavioural and neurological alterations associated with interruption of the 5-HT system after chronic depletion of dietary TRP. Therefore, Biggio et al. (1974) provided male Wistar rats with a diet devoid of TRP which resulted in a significant decrease of total and free TRP levels in serum $2 \mathrm{~h}$ after presentation of the diet. While total serum TRP levels decreased by 75\% compared to baseline levels, free TRP in serum decreased by about $90 \%$ and additionally correlated highly with the $85 \%$ decrease of TRP levels in the brain. Highly significant reductions were also found in 5-HT $(-58 \%)$ and 5-HIAA $(-76 \%)$ concentrations. The same group (Gessa et al., 1974) was also the first to demonstrate that peripheral TRP and brain TRP, 5-HT and 5-HIAA concentrations were significantly lower in rats orally administered (by gavage) a mixture of essential amino acids lacking TRP, compared to control rats that were administered an equivalent amount of distilled water. Training the rats to eat the same amount of food at a specific time point and within a limited time-frame (Biggio et al., 1974) could therefore be omitted and the straightforward method of acute tryptophan depletion as a serotonergic challenge tool was born. Not long thereafter, changes in the mental status of human subjects after the ingestion of an amino acid mixture devoid of TRP were evaluated for the first time. 


\section{Acute tryptophan depletion in humans}

The methodology of ATD as a serotonergic challenge tool, including theories regarding its underlying mechanism and its implication for psychiatry in general, have been widely explored and extensively reviewed over the past 20 years (Bell et al., 2005; Hood et al., 2005; Neumeister, 2003; Reilly et al., 1997; Young, 1993). Most researchers specifically address its potential as a tool to reveal depressive vulnerability and thus to predict the probability of a depressive episode (Booij et al., 2002; Booij et al., 2003; Booij et al., 2005b; Moreno et al., 1999; Moreno et al., 2000a; Neumeister et al., 1999) or a successful treatment response (Delgado et al., 1990; Delgado et al., 1991; Delgado et al., 1999). On the contrary, others question its validity and criticize its robustness as a tool to investigate the pathogenesis of depression (Norra, 2007; Ruhe et al., 2007).

\section{Acute tryptophan depletion; revealing vulnerability to depression}

Human subjects with genetic pre-existing 5-HT dysfunction may lack endogenous compensatory capacity to deal with an acute decrease in 5-HT metabolism, thereby exhibiting higher behavioural sensitivity to ATD (Jans et al., 2007b; Van der Does, 2001). This implies that a predisposition of so-called serotonergic vulnerability only results in direct overt psychiatric symptoms when these are triggered, as with ATD, by challenging the already vulnerable 5-HT system (Jans et al., 2007b) up to a certain threshold (Van der Does, 2001). In line with this hypothesis, ATD-induced transient mild mood-lowering effects, as reflected by lower mood ratings, have been reported in carriers of the "short" allelic polymorphism in the promoter of the 5-HT transporter gene 5-HTTLPR (SERT; Neumeister et al., 2002; Neumeister et al., 2006) and in healthy subjects with a family history of depression (Klaassen et al., 1999b). Likewise, a higher behavioural response to ATD has been observed in women (Ellenbogen et al., 1996; Smith et al., 1997), who are presumably predisposed to a lower 5-HT synthesis rate compared to men (Nishizawa et al., 1997). Moreover, ATD provokes a relapse of depressive symptoms in healthy subjects with a history of depression (Moreno et al., 1999; Neumeister et al., 2004). However, this effect is only in those previously treated successfully with selective serotonin re-uptake inhibitors (SSRIs) or MAO inhibiters (MAO-Is). Remitted, medication-free depressed patients with a positive treatment response history to antidepressants that primarily interact with systems other then 5-HT (e.g. tricyclic antidepressants or selective norepinephrine reuptake inhibitors) appear not to be affected by ATD (Delgado et al., 1990; Delgado et al., 1991; Delgado et al., 1999). The fact that ATD reverses the antidepressant response depending upon the specificity of the antidepressant treatment adds value to the challenge tool as a predictor of successful drug treatment response once serotonergic vulnerability has been revealed. 


\section{Acute tryptophan depletion in healthy subjects; inducing cognitive deficits}

It is generally believed and accepted that ATD does not induce considerable mood-lowering effects in healthy human subjects (Delgado et al., 1989; Moreno et al., 1999; Young et al., 1985). Nevertheless, acute decreased peripheral TRP levels and diminished 5-HIAA concentrations in CSF are consistently reported after ATD and appear to be similar in all subpopulations, i.e. in both healthy and so-called serotonergic vulnerable subjects (Carpenter et al., 1998; Evers et al., 2006; Klaassen et al., 1999b; Moore et al., 2000; Moreno et al., 2000b; Williams et al., 1999). This seems to be in line with the notion that both healthy and vulnerable subjects display cognitive dysfunctional behaviour after ATD as reported consistently between studies (Booij et al., 2005a; Evers et al., 2005; Klaassen et al., 1999a; Mendelsohn et al., 2009; Riedel et al., 1999; Riedel, 2004; Sambeth et al., 2007; Sambeth et al., 2009). It might therefore be suggested that an acute decrease in peripheral TRP levels directly interferes with mechanisms implicated in cognitive processing that depend less upon 5-HT functioning.

Altered cognitive processing has been reported with impairments in long term memory formation, decision making, reversal-learning and working memory. It is possible that an interruption in memory consolidation could underlie ATD-induced cognitive dysfunctions, as ATD has been shown to selectively impair memory consolidation in human subjects (Riedel et al., 1999). Memory consolidation is known to require brain-derived neurotrophic factor (BDNF; Lee et al., 2004), which is closely linked to the 5-HT system (Mamounas et al., 2000) and is most abundant in the hippocampus (Ernfors et al., 1990). Consequently, it has been suggested that ATD-induced impairment in memory consolidation is due to decreased 5-HT levels in hippocampal areas and linked to alterations in BDNF levels (see Riedel, 2004). Yet, such suggestions are likely to remain speculative as it is impossible to directly investigate the relationship between cognitive dysfunctional behaviour and acute neurochemical changes in the human brain in vivo.

\section{Acute tryptophan depletion in rats}

Animal models enable direct investigation of the relationships between brain and behaviour with the aim of gaining insight into human behaviour and its underlying neuronal and neuroendocrinological processes (van der Staay, 2006). Therefore, the direct consequences of ATD upon brain parameters like TRP and 5-HT in the rat can be used for appropriate interpretation of the alterations in behavioural output in accordance with the underlying neurochemical mechanism of the method. A large body of preclinical literature provides evidence that ATD in rats significantly depletes the levels of TRP in plasma, thereby reducing 5-HT metabolism, as is suggested by the lower TRP and 5-HT levels in rat brain tissue (Blokland et al., 2002; Cahir et al., 2007; Jans et al., 2007a; Lieben et al., 2004a; Olivier et al., 2008). ATD in rats consistently induces impairment in object memory performance (Jans et al., 2008a; Jans et 
al., 2007a; Jans and Blokland, 2008; Lieben et al., 2004b; Rutten et al., 2007; van Donkelaar et al., 2008) which, in contrast to human subjects, seems even more pronounced in rats with pre-existing abnormal 5-HT function (Olivier et al., 2008). Yet, in line with the ATD effects in healthy human subjects, ATDinduced alterations in affective behavioural parameters in the rat appear inconsistent (Blokland et al., 2002; Brown et al., 1998; Lieben et al., 2004b). Table 3 provides an overview of peripheral and central neurochemical effects, as well as cognitive and other behavioural alterations after ATD is induced in rats through administration of nutritional mixtures completely devoid of TRP.

\section{Solugel}

Reducing central 5-HT concentrations by lowering the levels of its dietary precursor TRP in plasma can be achieved by administration of specific TRPfree nutritional mixtures. Besides the TRP-free or TRP-low balanced diets and pure amino acid mixtures without TRP, a more advanced technique nowadays is the oral administration of a gelatin-based protein-carbohydrate mixture (Blokland et al., 2004). By adding a specific amount of TRP to the control mixture, effects of peripheral TRP suppletion, as often observed with traditional AA mixtures in humans (Fusar-Poli et al., 2006; Van der Does, 2001) are avoided and thus do not cause misinterpretation of the ATD effects (Blokland et al., 2004; Evers et al., 2005; Sambeth et al., 2009). Gelatin is derived from the selective hydrolysis of collagen protein, which is easily digestible and naturally lacks the essential amino acid TRP (Djagny et al., 2001). The gelatin hydrolysate used for the nutritional mixture is gelatin in an enzymatic hydrolyzed form, commercially available as Solugel ${ }^{\circledR}$ (PB Gelatins, Tessenderlo, Belgium). Solugel no longer consists of a combination of a few selective amino acids, but comprises a broad range of amino acids in the form of peptides, which makes it comparable to standard diets. Moreover, it is waterdispensable and unique for its gel-forming ability (Lieben et al., 2004a). In addition, a specific amount of carbohydrates are mixed with the Solugel, which adds an essential caloric value, thereby making the nutritional mixture even more similar to regular food intake. Moreover, mixing proteins with carbohydrates avoids any unwanted effects upon amino acid availability in the blood (see below and Figure 3) as is normally found with unbalanced diets containing high amounts of carbohydrates or proteins only (Benton, 2002; Markus et al., 1998; Markus, 2007). 


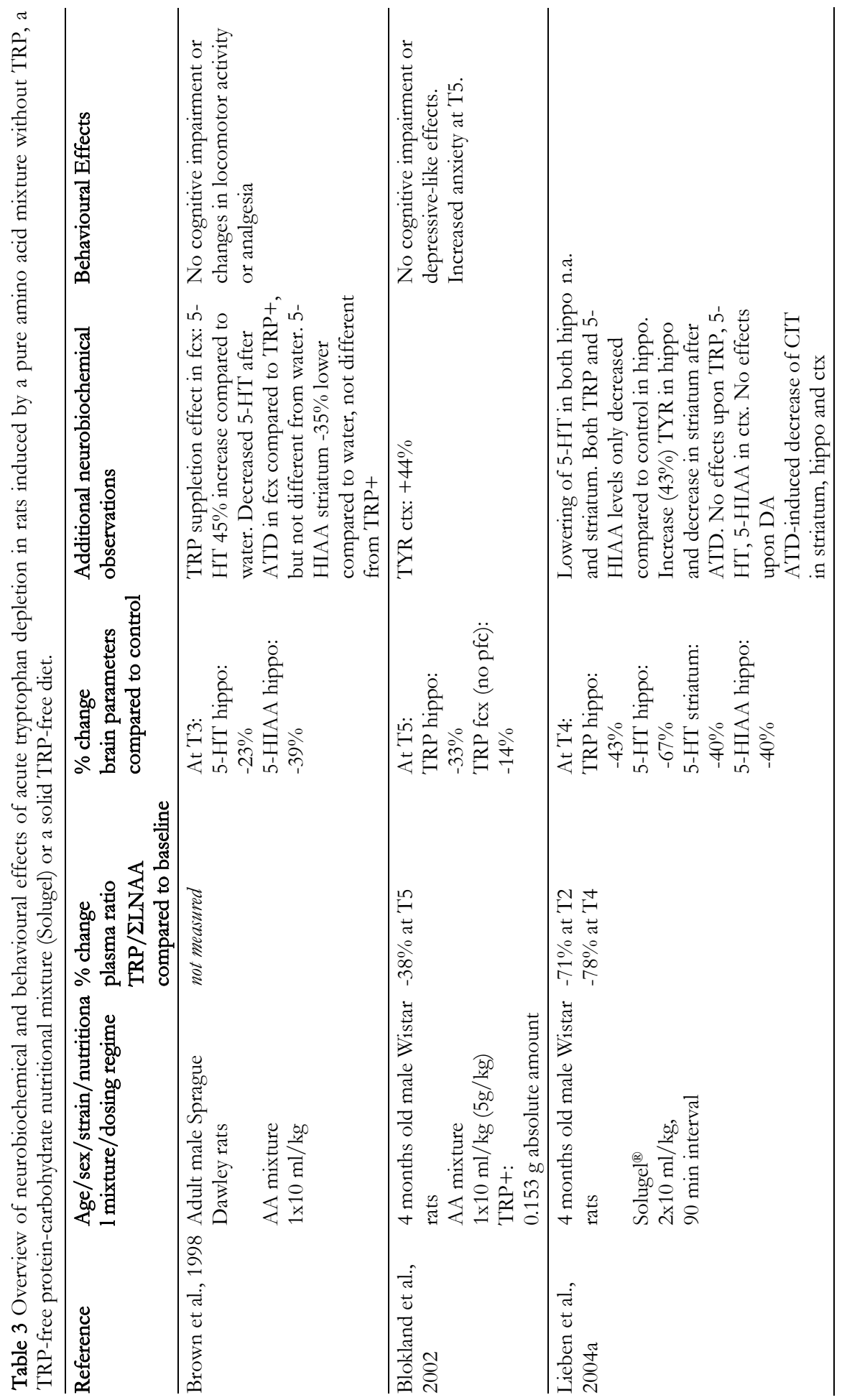




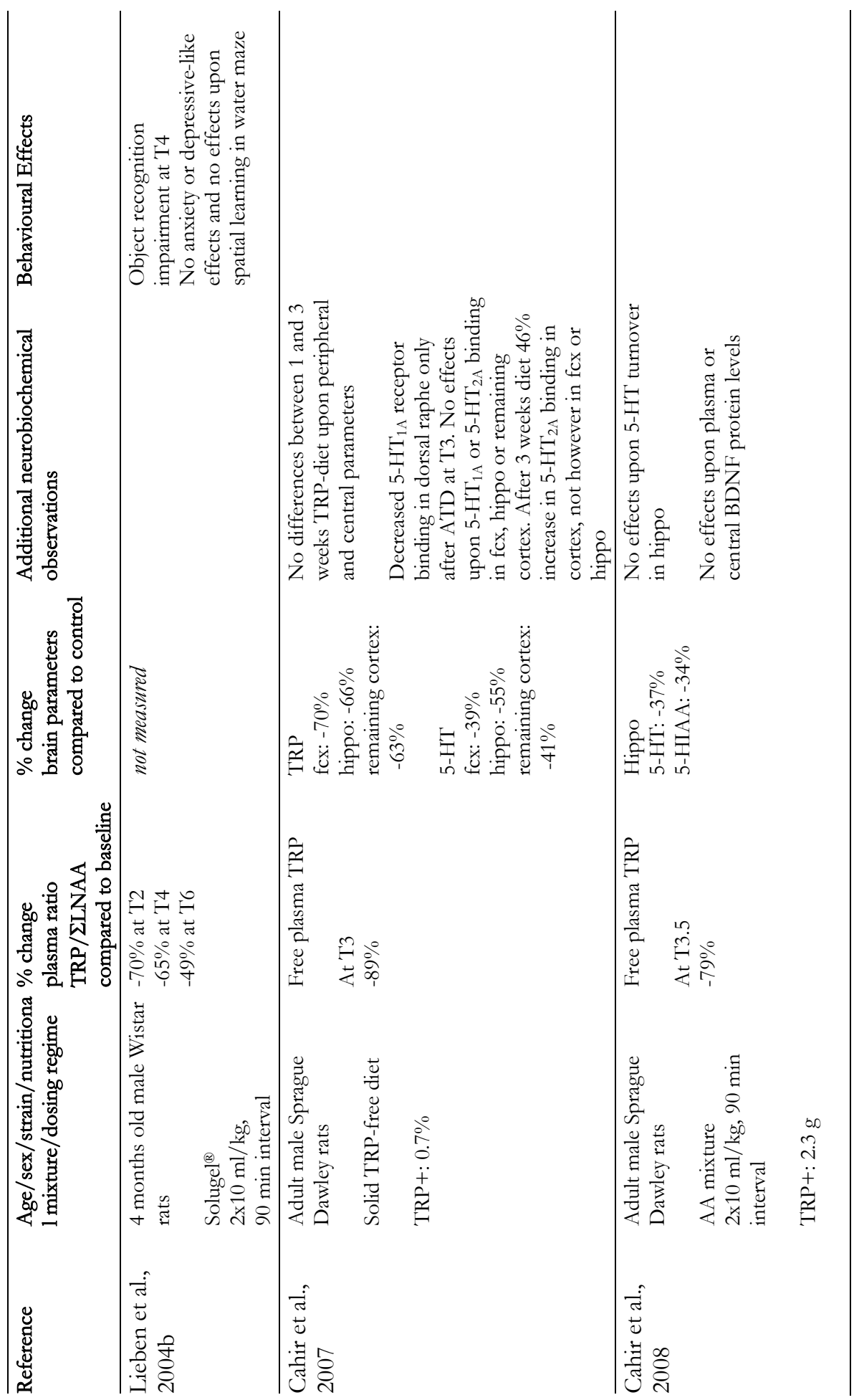




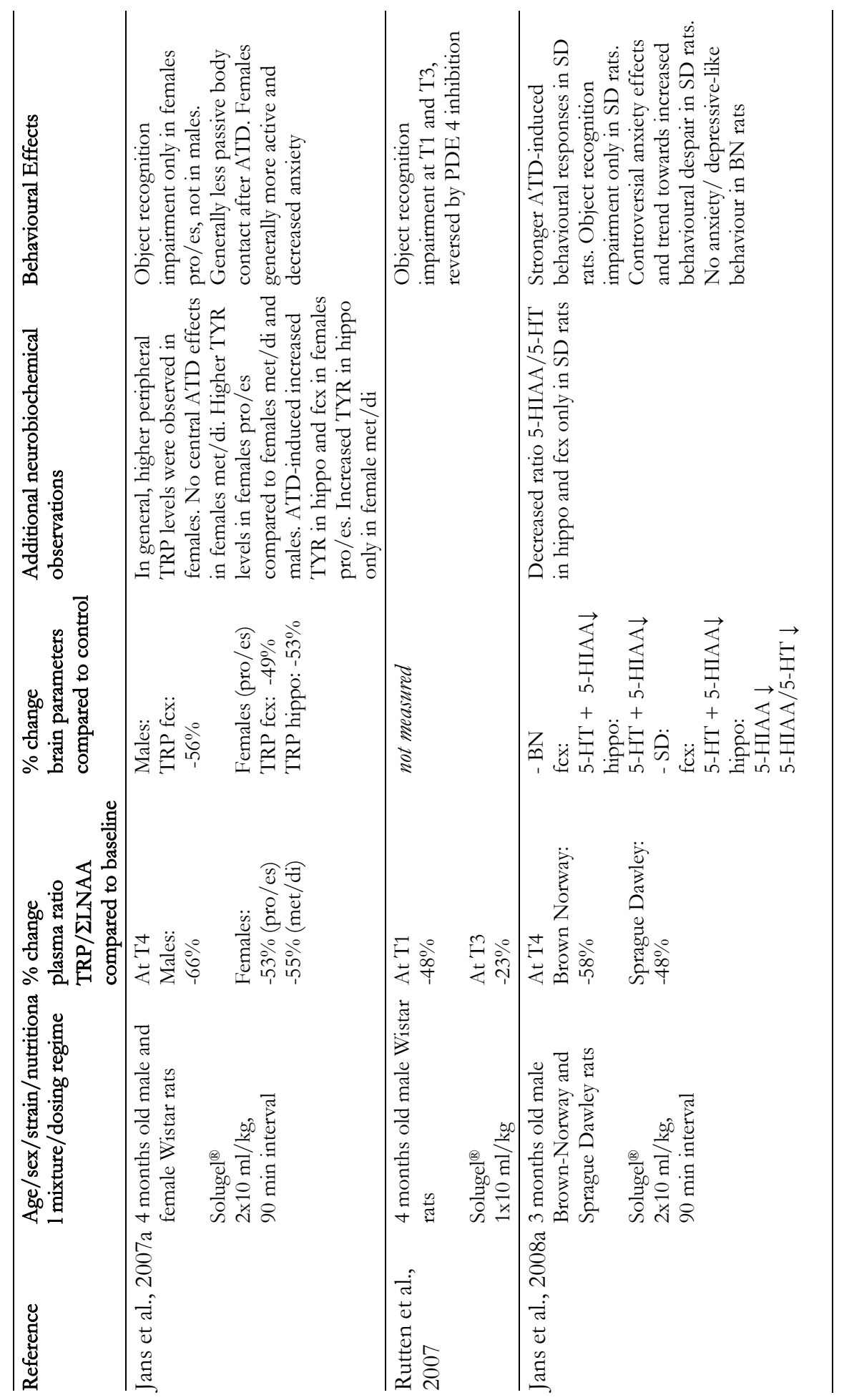




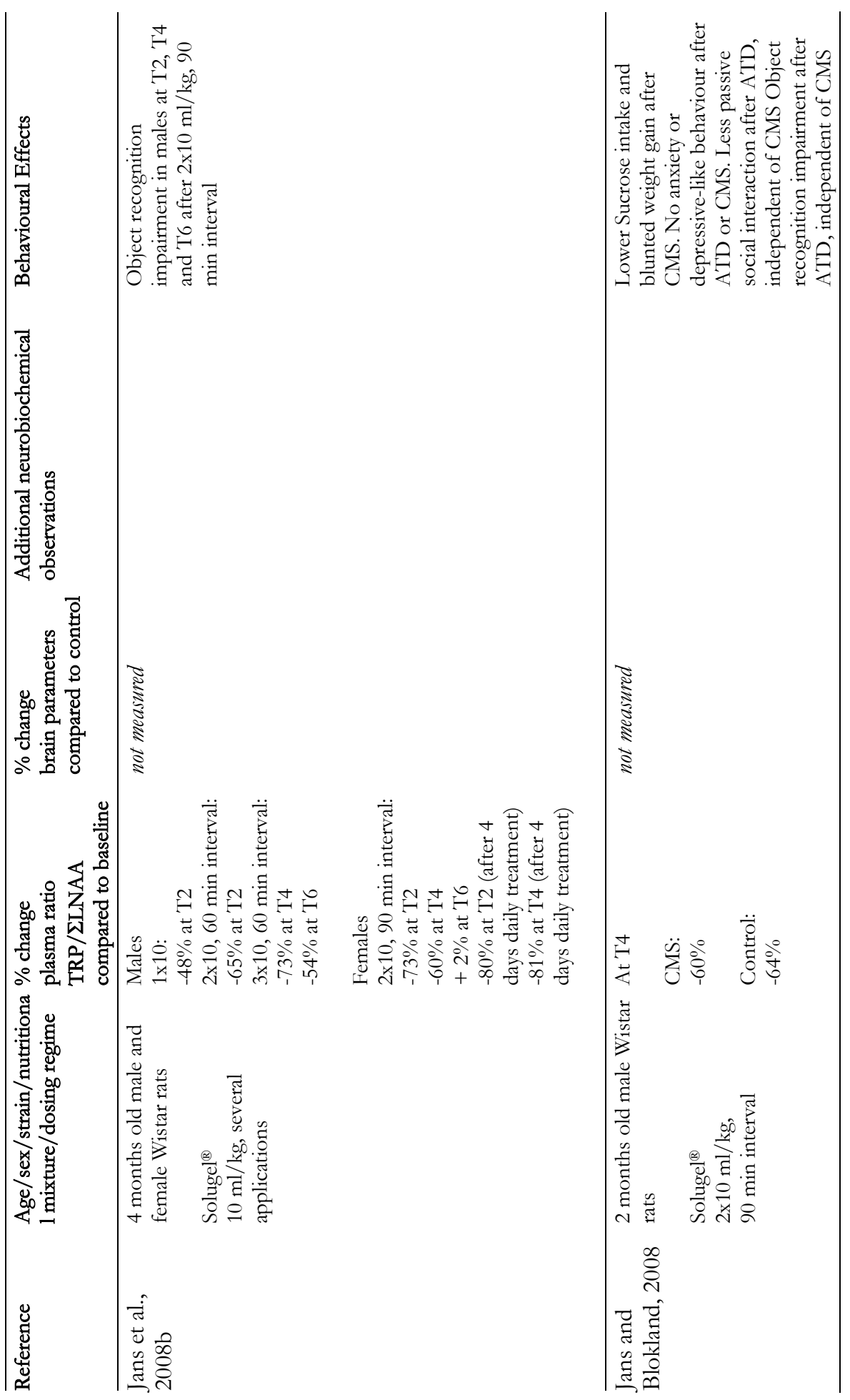




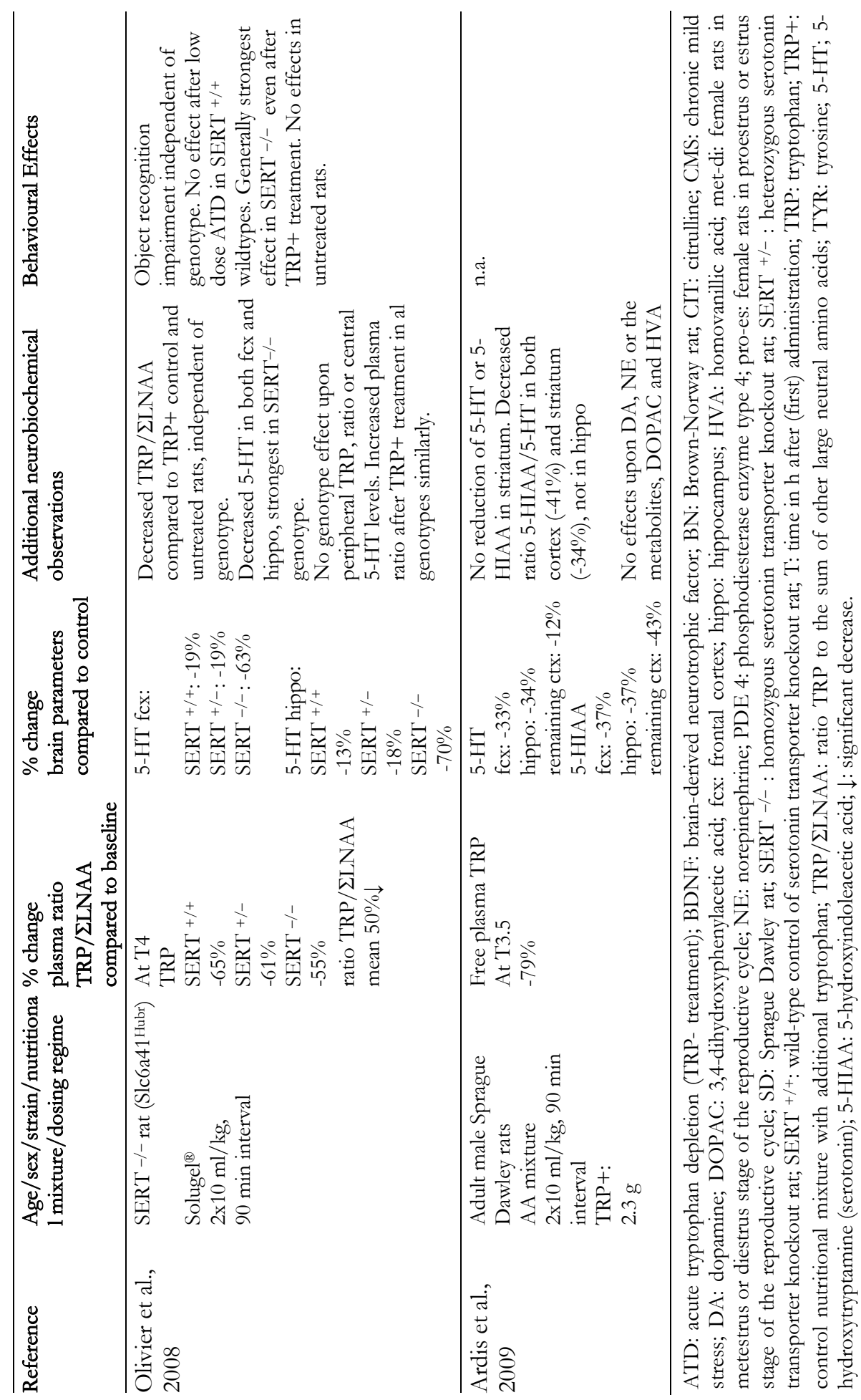




\section{Carbohydrates and dietary protein}

Carbohydrates raise blood glucose levels, thereby stimulating the pancreas to release insulin, an anabolic hormone. The secretion of insulin stimulates glucose to be taken up by the cells for subsequent normalization of glucose levels in the blood. Concomitantly with the insulin-induced drop in blood glucose levels, plasma TRP levels increase while the concentration of most other amino acids in plasma decreases (Fernstrom and Wurtman, 1972a). When a carbohydrate-rich meal contains no additional protein, activation of the insulin response increases protein synthesis, thereby stimulating the uptake of almost all amino acids (mainly the BCAAs) into muscle tissue. Most of the TRP in plasma is bound to serum albumin and thus not available for uptake into peripheral tissues, hence the effect of insulin upon TRP is much less compared to the other LNAAs. Moreover, insulin increases the affinity of serum albumin for TRP, thereby decreasing free circulating TRP and thus further increasing the ratio of bound to free plasma TRP. The increase in albumin affinity for TRP is a result of the rise in insulin which causes more unbound albumin to become available due to the stimulation of the uptake by fat cells of free fatty acids (NEFAs), which are normally bound to albumin. As TRP is the only amino acid bound to albumin, it is the only one highly prevented from being taken out of the bloodstream and up into peripheral tissue, specifically when the binding to albumin increases due to insulin secretion. Taken together, after the ingestion of carbohydrates the plasma ratio of LNAAs changes in favour of total TRP, which eventually leads to an increased availability of TRP in the brain to be synthesized into 5-HT (Fernstrom and Wurtman, 1972a; Fernstrom et al., 1987). Yet, as little as $2.5 \%$ of additional protein (by weight) is enough for the substantial increase of plasma LNAAs to counteract the effect of carbohydrates and the subsequent insulin-induced fall in LNAAs (Benton, 2002; Teff et al., 1989), as is the case with the TRP-free protein-carbohydrate nutritional mixture (see also Figure 3).

\section{Protein synthesis}

The administration of a TRP-free diet depletes plasma TRP acutely by inducing hepatic protein synthesis (Harper et al., 1970). In general, administration of a mixture lacking one essential amino acid results in the fall of the plasma level of that amino acid, which is rapidly removed from circulation by tissues to allow protein synthesis to proceed. Hence, a TRP-free diet induces a decrease of TRP levels in plasma due to a rapid removal of extracellular endogenous TRP from the circulation. 


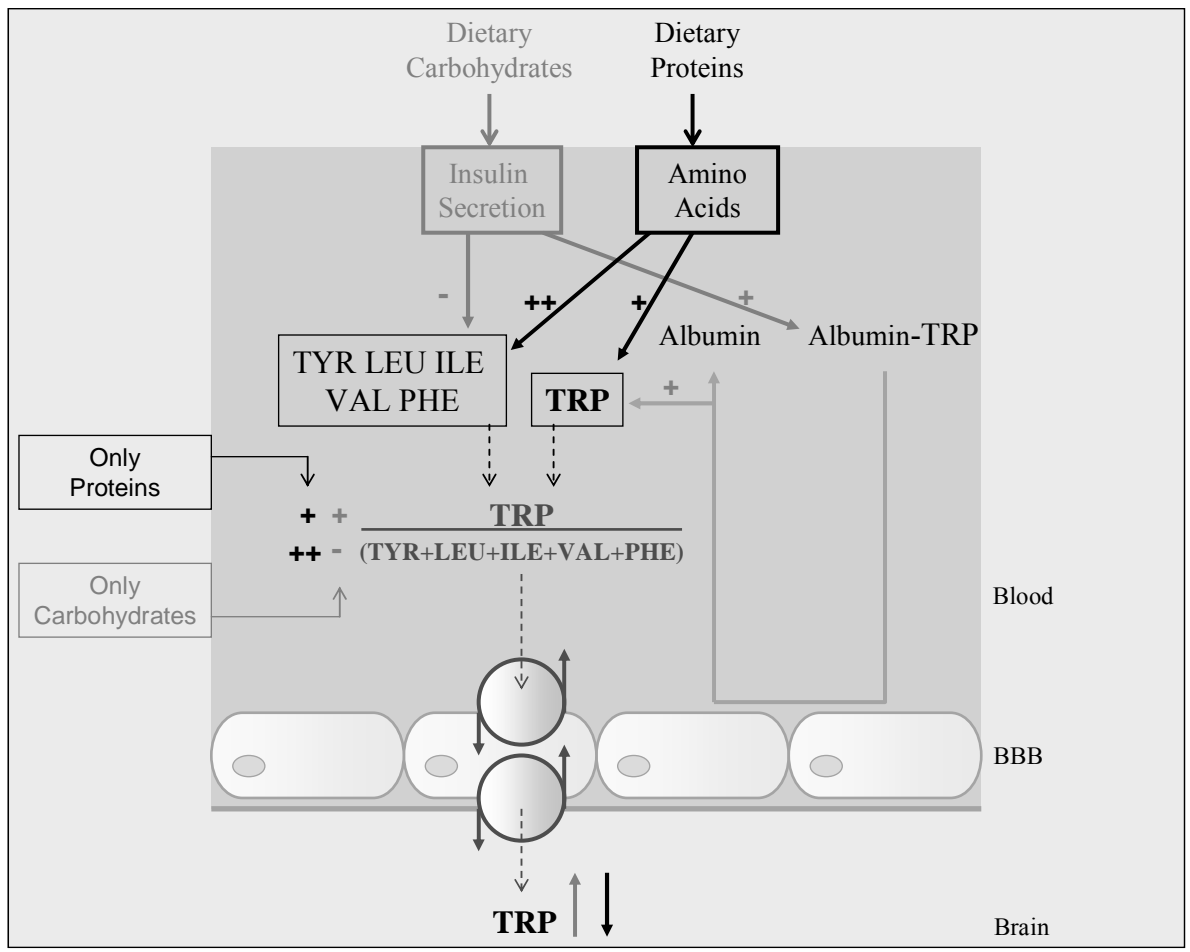

Figure 3 Differential effects of carbohydrates and protein upon the availability of TRP in plasma for uptake into the brain

Dietary carbohydrates increase the uptake of the large neutral amino acids (LNAAs; tyrosine [TYR], leucine [LEU], isoleucine [ILE], valine [VAL], phenylalaline [PHE]) into peripheral tissue, thereby decreasing their concentration in plasma. Together with an increase in total tryptophan (TRP) levels the ratio TRP to the sum of the LNAAs (TRP/ $\Sigma$ LNAA) changes in favour of TRP and increases its availability for transport across the blood-brain barrier (BBB). As little as $2.5 \%$ of additional proteins counteracts the effects of carbohydrates, as the protein ingestion-induced increase in the levels of all amino acids is much higher that the decrease by carbohydrates. When a TRP-free diet is administrated (acute tryptophan depletion), all amino acids are elevated except for TRP, thereby decreasing the ratio TRP/ $L$ LNAA and thus TRP uptake into the brain.

This extracellular TRP removal is due to an increased incorporation of TRP into proteins in liver and other tissue (Biggio et al., 1974; Gessa et al., 1974). The ATD-induced depletion of plasma TRP can be dose-dependently blocked by administration of the protein synthesis inhibitor cycloheximide together with a TRP-free diet (Gessa et al., 1975; Moja et al., 1991). Thus, protein synthesis, and not the inhibition of TRP transportation into the brain, seems to be the important initial mechanism underlying ATD-induced decreased 5-HT in the brain. 


\section{Challenging the serotonin system}

Since intact 5-HT neurotransmission is necessary for a wide range of physiological and functional processes, a disruption in this system can easily provoke diverse pathophysiological abnormalities, most of which are reflected in dysfunctional behavioural output. As ATD reduces 5-HT synthesis, ATDinduced behavioural changes in human subjects and laboratory animals are normally attributed to decreased 5-HT release, reflecting altered neuronal activity. For this reason, ATD has gained its popularity as non-toxic challenge to assess the implication of the 5-HT system in the pathogenesis and pathophysiology of psychiatric disorders. However, the exact mechanism by which ATD exerts its neurophysiological effects, and to what extent changes in 5-HT neuronal activity contribute to the ATD-induced neurophysiological and behavioural alterations, is not fully understood.

As previously outlined, brain concentrations of TRP are rate-limiting for 5-HT synthesis due to the fact that TPH is not saturated by the normal intracellular concentrations of TRP. Evidence of enhanced 5-HT synthesis after synthetic increases in brain TRP availability corroborate this notion (Fernstrom and Wurtman, 1971). Likewise, a variety of antidepressant drugs are thought to exert their therapeutic effect by increasing the levels of 5-HT concentration in the somatodendritic area, thereby increasing 5-HT neuronal release, i.e. activity. The increased 5-HT release promotes interaction with post-synaptic receptors, ultimately influencing behaviour. Along similar lines, it is generally believed that the mood-lowering and cognitive dysfunctional effects of ATD are mediated by decreases in 5-HT neuronal activity since a decrease in brain TRP availability reduces 5 -HT synthesis.

\section{The ratio TRP/ ILNAA in plasma and 5-HIAA levels in cerebrospinal fluid}

Effects of ATD treatment upon 5-HT levels in the brain cannot be directly investigated in humans. The ratio TRP/ $\Sigma$ LNAA in plasma indicates the amount of peripheral TRP potentially available for crossing the BBB, with a decrease in the ratio suggesting that less TRP will be available to the brain for synthesis into 5 -HT. This has been confirmed by the ATD-induced decrease in the uptake of central alpha-methyl-L-tryptophan ( $\alpha$-M-TRP) as measured by positron emission tomography (Nishizawa et al., 1997). Although this finding supports the concept that ATD exerts central effects, $\alpha$-MTrp uptake does not reveal anything specific about 5-HT release (Moore et al., 2000; Neumeister, 2003), despite the fact that it is considered a reliable indicator of 5-HT synthesis. Similarly, a decrease in the concentration of the inactive 5-HT metabolite 5-HIAA in CSF of humans subjected to ATD suggests that less 5-HT has been catabolyzed, which normally takes place after release, i.e. after neuronal firing. Therefore, a decrease in the amount of metabolite is thought to 
reflect decreased 5-HT metabolism, since lower intracellular 5-HT availability is presumed to result in less 5-HT release. However, numerous other peripheral factors seem to influence both the amount of 5-HIAA produced and its transport into and out of the CSF (see Cryan and Leonard, 2000). Despite the fact that both the ratio TRP/ $/ \mathrm{LNAA}$ and 5-HIAA concentrations are applied in human studies as indicators of altered 5-HT synthesis and metabolism, respectively, they do not allow any judgement concerning changes in 5-HT release or concomitant adaptations in neuronal activity.

\section{Central 5-HLA A levels and 5-HT turnover}

Animal studies not only provide the possibility to directly measure 5-HIAA levels in the specific brain areas, they also allow the calculation of the ratio of 5-HIAA to 5-HT concentrations. Alterations in the ratio 5-HIAA/5-HT are normally used to estimate changes in endogenous 5-HT turnover, which is thought to reflect 5-HT neuronal activity (Shannon et al., 1986). Unfortunately, ATD-induced changes in the ratio 5-HIAA/5-HT are controversially reported between studies (Ardis et al., 2009; Brown et al., 1998; Cahir et al., 2008; Jans et al., 2008a) and a decrease in the 5-HIAA/5-HT ratio after ATD is often difficult to directly attribute to a reduction in precursor (i.e. TRP) availability. An ATD-induced decrease of the ratio would only occur if significantly less 5-HIAA is produced compared to the already decreased amount of 5-HT synthesised. However, even then, a valid speculation upon changes in 5-HT turnover after ATD in rodents at one specific time-point is only possible if baseline measurements of central 5-HIAA and 5-HT concentrations are taken into account. Although both brain 5-HIAA concentrations and the ratio 5-HIAA/5-HT have been reported as valid indices of reduced 5-HT metabolism (Shannon et al., 1986), 5-HT neuronal activity can still only be estimated.

\section{5-HT neuronal release}

Direct evidence for alterations in neuronal activity comes from in vivo microdialysis measurements of changes in extracellular 5-HT concentrations, indicative of changes in neuronal release. Actual decreases in extracellular 5-HT have only been reported after ATD in combination with blockade of 5-HT reuptake (Bel and Artigas, 1996; Fadda et al., 2000; Stancampiano et al., 1997). A blockade after 5-HT reuptake seems necessary to raise 5-HT to optimal levels for detection (see Moore et al., 2000). Yet, without the initial systemic increase in 5-HT concentrations, basal 5-HT release in the prefrontal cortex of rats appeared not to be affected by ATD (van der Plasse et al., 2007). In addition decreased levels in whole brain 5-HT and 5-HIAA levels $2 \mathrm{~h}$ after the consumption of a TRP-free diet in cats did not parallel changes in the 
functional activity of 5-HT containing dorsal raphe cells throughout the $4 \mathrm{~h}$ after ingestion (Trulson, 1985).

Taken together, there is no direct evidence that brain 5-HT release, reflecting neuronal activity, is altered after ATD, whereas behavioural alterations can only be attributed to physiological changes in plasma TRP levels if the changes in brain 5-HT concentration actually reflect alterations in the functional activity of 5-HT neurons. Nevertheless, ATD consistently induces cognitive impairment in both animals and humans and triggers lowering of mood especially in healthy subjects predisposed with a vulnerable 5-HT system. ATD does not directly or indirectly affect other monoamine systems in rats (Ardis et al., 2009; Brown et al., 1998; Jans et al., 2008a; Lieben et al., 2004a) and no behavioural changes have been observed after depletion of other plasma amino acids (Klaassen et al., 1999a). Moreover, administration of a TRP-free AA mixture in primates decreased TRP and 5-HIAA concentrations in CBF without affecting catecholamine metabolites, suggesting that the catecholamine system is not influenced by ATD (Young et al., 1989). ATD-induced functional changes thus seem specific for the peripheral depletion of the essential amino acid TRP, but, are more likely 5-HT-mediated than 5-HT induced. In general, the moodlowering effects in human subjects with pre-existing abnormal 5-HT functioning, together with the controversial and diverse behavioural and biochemical preclinical results, suggest that ATD-induced alterations go beyond a straightforward dysfunction of the 5-HT system. Moreover, the effects of changes in substrate availability upon 5-HT synthesis rate are generally not conditioned under normal physiological circumstances, i.e. in the absence of any other regulatory changes. Therefore, changes other than substrate availability that interfere with normal 5-HT regulation or disruptions in substrate availability itself might act as potential confounding factors for the ATD-induced neurochemical effects as expected under normal circumstances (Lenard and Dunn, 2005). How then should one interpret the behavioural changes after ATD and what are the consequences for speculation upon the implication of the 5-HT system in depressive disorders? 


\section{This thesis}

\section{A mechanistic approach to the method of acute tryptophan depletion in rodents}

The method of ATD is non-toxic and reversible and can thus be used safely and repeatedly in both human subjects and rodents, thereby offering the ability to compare between clinical and preclinical experimental designs and outcomes. However, the neurophysiological mechanisms underlying behaviour cannot be as directly investigated in humans as they can in laboratory animals. Associating acute neurophysiological alterations to behavioural output may provide more insight into the extent to which ATD-induced behavioural dysfunction can be directly attributed to presumed changes in 5-HT neuronal activity. Not only is the 5-HT system involved in the modulation of many important behavioural and physiological functions, depression is also a heterogeneous disorder with a complex and as yet unresolved underlying pathophysiology. In this sense, challenging the serotonergic system by means of ATD and linking neurophysiological changes to behavioural output in light of clinical depressive symptomatology might seem somewhat challenging itself. Yet, a mechanistic approach to the method of ATD in rodents through exploration of its underlying neurobiochemical mechanisms will not only improve the interpretation of clinical ATD-induced symptoms, but will also provide new insights into the implication of the 5-HT system in depressive and other neurobiological brain disorders. This thesis covers a comprehensive evaluation of the diverse and sometimes controversial neurochemical and behavioural effects of ATD in rodents and aims to explore potential mechanisms by which ATD exerts its neurophysiological and behavioural effects.

\section{Outline}

Pre-existing dysfunctional 5-HT systems are nowadays widely modeled in mice by manipulating genes implicated in the regulation of the 5-HT system. The introduction of these new mouse models of depression appear to be extremely important to improve our insight in the underlying neurophysiological mechanisms of depressive disorders and the development of new pharmacological therapies. Consequently, the use of mice instead of rats for studying behaviour and the implication of neurotransmitter systems in psychiatric disorders in general has significantly increased. It was, therefore, initially aimed to establish the ATD method in the mouse in order to be able to apply the method in transgenic mouse models that are predisposed to serotonergic dysfunction. Chapter 2 provides an overview of the multiple studies in which the pharmacokinetic and behavioural effects of different dosing regimes of the TRP-free protein-carbohydrate mixture were explored, particularly in the C57BL/ 6 mouse. 
In rats, ATD has been extensively used and the TRP-free protein-carbohydrate mixture has been proved to successfully lower peripheral TRP levels, thereby interfering with 5-HT metabolism and presumably 5-HT neuronal activity in the rat brain. Under normal physiological conditions, neuronal activity is related to the cerebral metabolic rate of glucose (CMRG), which is in turn closely coupled to cerebral blood flow (CBF). As the 5-HT neurotransmitter additionally possesses important vasoactive properties, an acute decrease in central 5-HT concentration could alter CMRG and $\mathrm{CBF}$ and the normal relationship between them. Such cerebrovascular alterations may contribute to the underlying mechanisms of ATD-induced affective behavioural changes and cognitive impairments. Chapter 3 describes the effects of ATD upon local CBF and $\mathrm{CMRG}$ as measured in 43 distinct brain regions of adult male Wistar rats by means of $\left[{ }^{14} \mathrm{C}\right]$-iodoantipyrine and $\left[{ }^{14} \mathrm{C}\right]$-2-deoxyglucose quantitative autoradiographic imaging, respectively.

An acute decrease in brain 5-HT is likely to result in disturbed affective behaviour only if there is pre-existing vulnerability in the 5-HT system. The neurotoxicity of MDMA is known to cause significant 5-HT terminal depletion, thereby decreasing 5-HT concentrations in the brain. In Chapter 4, adult male Wistar rats are repeatedly pretreated with MDMA in order to apply ATD in an animal model of acquired 5-HT dysfunction. By using quantitative autoradiographic imaging, the effects of repeated MDMA pretreatment upon local CBF and CMRG are measured under an ATD challenged condition, thereby investigating the relationship between cerebrovascular function and 5-HT changes in general and MDMA-induced 5-HT dysfunction as a vulnerability factor for depression in general, and cognitive dysfunction in particular.

BDNF is strongly linked to the 5-HT system and, besides being extremely stress-responsive, plays an essential role in mood and memory processes. To explore the potential role of BDNF in the underlying mechanisms of ATDinduced behavioural changes, Chapter 5 examines the effects of ATD upon BDNF in serum, hippocampus and prefrontal cortex of adult male Wistar rats, while controlling for possible confounding stress effects of the specific experimental procedures.

Whereas affective behavioural changes after ATD remain controversial, ATD-induced memory deficits are consistently reported in humans, but also in rats as measured by means of the rat object recognition task. Inhibition of phosphodiesterase (PDE) enzymes appears to be a reliable method to improve memory processes by increasing the levels of the second messenger molecules cyclic adenosine monophosphate (cAMP) and cyclic guanosine monophosphate (cGMP) or both. Chapter 6 examines the ability of the PDE inhibitors vardenafil and BAY 60-7550, that increase cAMP and both cAMP and cGMP, respectively, to reverse an ATD-induced impairment in object recognition performance of adult male Wistar rats. Exploring the pro-cognitive effects of PDE inhibitors might provide more insight into a potential interaction between 
second messenger molecules and the 5-HT system as an underlying mechanism for ATD-induced cognitive dysfunction.

\section{References}

Ardis TC, Cahir M, Elliott JJ, Bell R, Reynolds GP, Cooper SJ (2009). Effect of acute tryptophan depletion on noradrenaline and dopamine in the rat brain. J Psychopharmacol 23(1): 51-55.

Asberg M, Thoren P, Traskman L, Bertilsson L, Ringberger V (1976a). "Serotonin depression"- a biochemical subgroup within the affective disorders? Science 191(4226): 478-480.

Asberg M, Traskman L, Thoren P (1976b). 5-HIAA in the cerebrospinal fluid. A biochemical suicide predictor? Arch Gen Psychiatry 33(10): 1193-1197.

Badawy AB (2009). Plasma free tryptophan revisited: what you need to know and do before measuring it. J Psychopharmacol.

Bel N, Artigas F (1996). Reduction of serotonergic function in rat brain by tryptophan depletion: effects in control and fluvoxamine-treated rats. J Neurochem 67(2): 669-676.

Bell CJ, Hood SD, Nutt DJ (2005). Acute tryptophan depletion. Part II: clinical effects and implications. Aust N Z J Psychiatry 39(7): 565-574.

Bender DA (1983). Biochemistry of tryptophan in health and disease. Mol Aspects Med 6(2): 101-197.

Benton D (2002). Carbohydrate ingestion, blood glucose and mood. Neurosci Biobehav Rev 26(3): 293-308.

Biggio G, Fadda F, Fanni P, Tagliamonte A, Gessa GL (1974). Rapid depletion of serum tryptophan, brain tryptophan, serotonin and 5-hydroxyindoleacetic acid by a tryptophan-free diet. Life Sci 14(7): 1321-1329.

Blokland A, Lieben C, Deutz NE (2002). Anxiogenic and depressive-like effects, but no cognitive deficits, after repeated moderate tryptophan depletion in the rat. J Psychopharmacol 16(1): 39-49.

Blokland A, Lieben C, Deutz NEP, Schmitt J (2004). Acute Tryptophan depletion: comparing the effects of an amino acid mixture with a gelatin-based protein in man and rats. Current Topics in Nutraceutical Research 2(3): 1-8.

Boadle-Biber MC (1993). Regulation of serotonin synthesis. Prog Biophys Mol Biol 60(1): 1-15.

Booij L, Van der Does W, Benkelfat C, Bremner JD, Cowen PJ, Fava M, Gillin C, Leyton M, Moore P, Smith KA, Van der Kloot WA (2002). Predictors of mood response to acute tryptophan depletion. A reanalysis. Neuropsychopharmacology 27(5): 852-861.

Booij L, Van der Does AJ, Riedel WJ (2003). Monoamine depletion in psychiatric and healthy populations: review. Mol Psychiatry 8(12): 951-973.

Booij L, Van der Does AJ, Haffmans PM, Riedel WJ, Fekkes D, Blom MJ (2005a). The effects of high-dose and low-dose tryptophan depletion on mood and cognitive functions of remitted depressed patients. J Psychopharmacol 19(3): 267-275.

Booij L, van der Does AJ, Haffmans PM, Spinhoven P, McNally RJ (2005b). Acute tryptophan depletion as a model of depressive relapse: behavioural specificity and ethical considerations. Br J Psychiatry 187: 148-154.

Botting NP (1995). Chemistry and neurochemistry of the kynurenine pathway of tryptophan metabolism. Chem Soc Rev 24: 401-412.

Brown CM, Fletcher PJ, Coscina DV (1998). Acute amino acid loads that deplete brain serotonin fail to alter behavior. Pharmacology, biochemistry, and behavior 59(1): 115-121.

Cahir M, Ardis T, Reynolds GP, Cooper SJ (2007). Acute and chronic tryptophan depletion differentially regulate central 5-HT1A and 5-HT $2 \mathrm{~A}$ receptor binding in the rat. Psychopharmacology 190(4): 497-506.

Cahir M, Ardis TC, Elliott JJ, Kelly CB, Reynolds GP, Cooper SJ (2008). Acute tryptophan depletion does not alter central or plasma brain-derived neurotrophic factor in the rat. Eur Neuropsychopharmacol 18(5): 317-322. 
Carpenter LL, Anderson GM, Pelton GH, Gudin JA, Kirwin PD, Price LH, Heninger GR, McDougle CJ (1998). Tryptophan depletion during continuous CSF sampling in healthy human subjects. Neuropsychopharmacology 19(1): 26-35.

Cowen PJ, Parry-Billings M, Newsholme EA (1989). Decreased plasma tryptophan levels in major depression. J Affect Disord 16(1): 27-31.

Crane GE (1957). Iproniazid (marsilid) phosphate, a therapeutic agent for mental disorders and debilitating diseases. Psychiatr Res Rep Am Psychiatr Assoc 8: 142-152.

Cryan JF, Leonard BE (2000). 5-HT1A and beyond: the role of serotonin and its receptors in depression and the antidepressant response. Hum Psychopharmacol 15(2): 113-135.

Curzon G, Friedel J, Knott PJ (1973). The effect of fatty acids on the binding of tryptophan to plasma protein. Nature 242(5394): 198-200.

Curzon G, Knott PJ (1973). Drugs influencing plasma and brain tryptophan. Br J Pharmacol 48(2): 352P-353P.

Delgado PL, Charney DS, Price LH, Landis H, Heninger GR (1989). Neuroendocrine and behavioral effects of dietary tryptophan restriction in healthy subjects. Life Sci 45(24): 23232332.

Delgado PL, Charney DS, Price LH, Aghajanian GK, Landis H, Heninger GR (1990). Serotonin function and the mechanism of antidepressant action. Reversal of antidepressant-induced remission by rapid depletion of plasma tryptophan. Arch Gen Psychiatry 47(5): 411-418.

Delgado PL, Price LH, Miller HL, Salomon RM, Licinio J, Krystal JH, Heninger GR, Charney DS (1991). Rapid serotonin depletion as a provocative challenge test for patients with major depression: relevance to antidepressant action and the neurobiology of depression. Psychopharmacol Bull 27(3): 321-330.

Delgado PL, Miller HL, Salomon RM, Licinio J, Krystal JH, Moreno FA, Heninger GR, Charney DS (1999). Tryptophan-depletion challenge in depressed patients treated with desipramine or fluoxetine: implications for the role of serotonin in the mechanism of antidepressant action. Biol Psychiatry 46(2): 212-220.

Djagny VB, Wang Z, Xu S (2001). Gelatin: a valuable protein for food and pharmaceutical industries: review. Crit Rev Food Sci Nutr 41(6): 481-492.

DSM-IV-TR. 2000. Diagnostic and Statistical Manual of Mental Disorders Text Revision, 4th edn. American Psychiatric Association, Washington, DC.

Ellenbogen MA, Young SN, Dean P, Palmour RM, Benkelfat C (1996). Mood response to acute tryptophan depletion in healthy volunteers: sex differences and temporal stability. Neuropsychopharmacology 15(5): 465-474.

Ernfors P, Wetmore C, Olson L, Persson H (1990). Identification of cells in rat brain and peripheral tissues expressing mRNA for members of the nerve growth factor family. Neuron 5(4): 511-526.

Erspamer V, Vialli M (1937). Ricerche sul secreto delle cellule enterocromaffini. Boll Soc Medchir Pavia 51: 357-363.

Evers EA, Tillie DE, van der Veen FM, Lieben CK, Jolles J, Deutz NE, Schmitt JA (2005). Effects of a novel method of acute tryptophan depletion on plasma tryptophan and cognitive performance in healthy volunteers. Psychopharmacology 178(1): 92-99.

Evers EA, van der Veen FM, Jolles J, Deutz NE, Schmitt JA (2006). Acute tryptophan depletion improves performance and modulates the BOLD response during a Stroop task in healthy females. Neuroimage 32(1): 248-255.

Fadda F, Cocco S, Stancampiano R (2000). A physiological method to selectively decrease brain serotonin release. Brain Res Brain Res Protoc 5(3): 219-222.

Fernstrom JD, Wurtman RJ (1971). Brain serotonin content: physiological dependence on plasma tryptophan levels. Science 173(992): 149-152.

Fernstrom JD, Wurtman RJ (1972a). Elevation of plasma tryptophan by insulin in rat. Metabolism 21(4): 337-342.

Fernstrom JD, Wurtman RJ (1972b). Brain serotonin content: physiological regulation by plasma neutral amino acids. Science 178(59): 414-416. 
Fernstrom JD (1979a). Comment on the methodology for separating and determining free and albumin-bound tryptophan levels in blood samples. J Neural Transm Suppl(15): 217-219.

Fernstrom JD (1979b). Diet-induced changes in plasma amino acid pattern: effects on the brain uptake of large neutral amino acids, and on brain serotonin synthesis. J Neural Transm Suppl(15): 55-67.

Fernstrom JD (1983). Role of precursor availability in control of monoamine biosynthesis in brain. Physiol Rev 63(2): 484-546.

Fernstrom JD, Fernstrom MH, Grubb PE (1987). Twenty-four-hour variations in rat blood and brain levels of the aromatic and branched-chain amino acids: chronic effects of dietary protein content. Metabolism 36(7): 643-650.

France CM, Lysaker PH, Robinson RP (2007). The "chemical imbalance" explanation for depression: origions, lay endorsement, and clinical implications. Prof Psychol Res Pr 38(4): 411-420.

Fusar-Poli P, Allen P, McGuire P, Placentino A, Cortesi M, Perez J (2006). Neuroimaging and electrophysiological studies of the effects of acute tryptophan depletion: a systematic review of the literature. Psychopharmacology (Berl) 188(2): 131-143.

Gessa GL, Biggio G, Fadda F, Corsini GU, Tagliamonte A (1974). Effect of the oral administration of tryptophan-free amino acid mixtures on serum tryptophan, brain tryptophan and serotonin metabolism. J Neurochem 22(5): 869-870.

Gessa GL, Tagliamonte A (1974). Possible role of free serum tryptophan in the control of brain tryptophan level and serotonin synthesis. Adv Biochem Psychopharmacol 11(0): 119-131.

Gessa GL, Biggio G, Fadda F, Corsini GU, Tagliamonte A (1975). Tryptophan-free diet: a new means for rapidly decreasing brain tryptophan content and serotonin synthesis. Acta Vitaminol Enzymol 29(1-6): 72-78.

Harper AE, Benevenga NJ, Wohlhueter RM (1970). Effects of ingestion of disproportionate amounts of amino acids. Physiol Rev 50(3): 428-558.

Hindmarch I (2002). Beyond the monoamine hypothesis: mechanisms, molecules and methods. Eur Psychiatry 17 Suppl 3: 294-299.

Hood SD, Bell CJ, Nutt DJ (2005). Acute tryptophan depletion. Part I: rationale and methodology. The Australian and New Zealand journal of psychiatry 39(7): 558-564.

$\mathrm{Hu}$ B, Hissong BD, Carlin JM (1995). Interleukin-1 enhances indoleamine 2,3-dioxygenase activity by increasing specific mRNA expression in human mononuclear phagocytes. J Interferon Cytokine Res 15(7): 617-624.

Jans L, Korte-Bouws G, Korte S, Blokland A (2008a). The effects of acute tryptophan depletion on affective behaviour and cognition in Brown Norway and Sprague Dawley rats. J Psychopharmacol.

Jans LA, Lieben CK, Blokland A (2007a). Influence of sex and estrous cycle on the effects of acute tryptophan depletion induced by a gelatin-based mixture in adult Wistar rats. Neuroscience 147(2): 304-317.

Jans LA, Riedel WJ, Markus CR, Blokland A (2007b). Serotonergic vulnerability and depression: assumptions, experimental evidence and implications. Mol Psychiatry 12(6): 522-543.

Jans LA, Blokland A (2008). Influence of chronic mild stress on the behavioural effects of acute tryptophan depletion induced by a gelatin-based mixture. Behav Pharmacol 19(7): 706-715.

Jans LA, Lieben CK, Smits LT, Blokland A (2008b). Pharmacokinetics of acute tryptophan depletion using a gelatin-based protein in male and female Wistar rats. Amino Acids.

Klaassen T, Riedel WJ, Deutz NE, van Someren A, van Praag HM (1999a). Specificity of the tryptophan depletion method. Psychopharmacology 141(3): 279-286.

Klaassen T, Riedel WJ, van Someren A, Deutz NE, Honig A, van Praag HM (1999b). Mood effects of 24-hour tryptophan depletion in healthy first-degree relatives of patients with affective disorders. Biol Psychiatry 46(4): 489-497.

Kuhn R (1958). The treatment of depressive states with G 22355 (imipramine hydrochloride). Am J Psychiatry 115(5): 459-464.

Lee JL, Everitt BJ, Thomas KL (2004). Independent cellular processes for hippocampal memory consolidation and reconsolidation. Science 304(5672): 839-843. 
Lenard NR, Dunn AJ (2005). Mechanisms and significance of the increased brain uptake of tryptophan. Neurochemical research 30(12): 1543-1548.

Leonard BE. 1997. Fundamentals of Psychopharmacology. New York: Wiley and Sons.

Leonard BE (2007). Psychopathology of depression. Drugs of today (Barcelona, Spain: 1998) 43(10): 705-716.

Lieben CK, Blokland A, Westerink B, Deutz NE (2004a). Acute tryptophan and serotonin depletion using an optimized tryptophan-free protein-carbohydrate mixture in the adult rat. Neurochem Int 44(1): 9-16.

Lieben CK, van Oorsouw K, Deutz NE, Blokland A (2004b). Acute tryptophan depletion induced by a gelatin-based mixture impairs object memory but not affective behavior and spatial learning in the rat. Behav Brain Res 151(1-2): 53-64.

Maes M, Meltzer HY. 1995. The serotonin hypothesis of major depression. E. BF, D.J. K, editors. New York: Raven Press, Ltd. 933-944 p.

Mamounas LA, Altar CA, Blue ME, Kaplan DR, Tessarollo L, Lyons WE (2000). BDNF promotes the regenerative sprouting, but not survival, of injured serotonergic axons in the adult rat brain. J Neurosci 20(2): 771-782.

Markus CR, Panhuysen G, Tuiten A, Koppeschaar H, Fekkes D, Peters ML (1998). Does carbohydrate-rich, protein-poor food prevent a deterioration of mood and cognitive performance of stress-prone subjects when subjected to a stressful task? Appetite 31(1): 4965.

Markus CR (2007). Effects of carbohydrates on brain tryptophan availability and stress performance. Biol Psychol 76(1-2): 83-90.

Meltzer HY (1990). Role of serotonin in depression. Ann N Y Acad Sci 600: 486-499; discussion 499-500.

Mendelsohn D, Riedel WJ, Sambeth A (2009). Effects of acute tryptophan depletion on memory, attention and executive functions: a systematic review. Neurosci Biobehav Rev 33(6): 926952.

Moja EA, Restani P, Corsini E, Stacchezzini MC, Assereto R, Galli CL (1991). Cycloheximide blocks the fall of plasma and tissue tryptophan levels after tryptophan-free amino acid mixtures. Life Sci 49(15): 1121-1128.

Moore P, Landolt HP, Seifritz E, Clark C, Bhatti T, Kelsoe J, Rapaport M, Gillin JC (2000). Clinical and physiological consequences of rapid tryptophan depletion. Neuropsychopharmacology 23(6): 601-622.

Moreno FA, Gelenberg AJ, Heninger GR, Potter RL, McKnight KM, Allen J, Phillips AP, Delgado PL (1999). Tryptophan depletion and depressive vulnerability. Biol Psychiatry 46(4): 498-505.

Moreno FA, Heninger GR, McGahuey CA, Delgado PL (2000a). Tryptophan depletion and risk of depression relapse: a prospective study of tryptophan depletion as a potential predictor of depressive episodes. Biol Psychiatry 48(4): 327-329.

Moreno FA, McGavin C, Malan TP, Gelenberg AJ, Heninger GR, Mathe AA, Delgado PL (2000b). Tryptophan depletion selectively reduces CSF 5-HT metabolites in healthy young men: results from single lumbar puncture sampling technique. Int J Neuropsychopharmacol 3(4): 277-283.

Moroni F (1999). Tryptophan metabolism and brain function: focus on kynurenine and other indole metabolites. Eur J Pharmacol 375(1-3): 87-100.

Murray CJ, Lopez AD (1997). Alternative projections of mortality and disability by cause 19902020: Global Burden of Disease Study. Lancet 349(9064): 1498-1504.

Naughton M, Mulrooney JB, Leonard BE (2000). A review of the role of serotonin receptors in psychiatric disorders. Hum Psychopharmacol 15(6): 397-415.

Nestler EJ, Gould E, Manji H, Buncan M, Duman RS, Greshenfeld HK, Hen R, Koester S, Lederhendler I, Meaney M, Robbins T, Winsky L, Zalcman S (2002). Preclinical models: status of basic research in depression. Biol Psychiatry 52(6): 503-528. 
Neumeister A, Habeler A, Praschak-Rieder N, Willeit M, Kasper S (1999). Tryptophan depletion: a predictor of future depressive episodes in seasonal affective disorder? Int Clin Psychopharmacol 14(5): 313-315.

Neumeister A, Konstantinidis A, Stastny J, Schwarz MJ, Vitouch O, Willeit M, Praschak-Rieder N, Zach J, de Zwaan M, Bondy B, Ackenheil M, Kasper S (2002). Association between serotonin transporter gene promoter polymorphism (5HTTLPR) and behavioral responses to tryptophan depletion in healthy women with and without family history of depression. Arch Gen Psychiatry 59(7): 613-620.

Neumeister A (2003). Tryptophan depletion, serotonin, and depression: where do we stand? Psychopharmacol Bull 37(4): 99-115.

Neumeister A, Nugent AC, Waldeck T, Geraci M, Schwarz M, Bonne O, Bain EE, Luckenbaugh DA, Herscovitch P, Charney DS, Drevets WC (2004). Neural and behavioral responses to tryptophan depletion in unmedicated patients with remitted major depressive disorder and controls. Arch Gen Psychiatry 61(8): 765-773.

Neumeister A, Hu XZ, Luckenbaugh DA, Schwarz M, Nugent AC, Bonne O, Herscovitch P, Goldman D, Drevets WC, Charney DS (2006). Differential effects of 5-HTTLPR genotypes on the behavioral and neural responses to tryptophan depletion in patients with major depression and controls. Arch Gen Psychiatry 63(9): 978-986.

Nishizawa S, Benkelfat C, Young SN, Leyton M, Mzengeza S, de Montigny C, Blier P, Diksic M (1997). Differences between males and females in rates of serotonin synthesis in human brain. Proc Natl Acad Sci U S A 94(10): 5308-5313.

Norra C (2007). Challenge tests of monoaminergic systems: neurophysiological aspects. Clin EEG Neurosci 38(2): 66-73.

Oldendorf WH (1971). Brain uptake of radiolabeled amino acids, amines, and hexoses after arterial injection. Am J Physiol 221(6): 1629-1639.

Oldendorf WH, Szabo J (1976). Amino acid assignment to one of three blood-brain barrier amino acid carriers. Am J Physiol 230(1): 94-98.

Olivier JD, Jans LA, Korte-Bouws GA, Korte SM, Deen PM, Cools AR, Ellenbroek BA, Blokland A (2008). Acute tryptophan depletion dose dependently impairs object memory in serotonin transporter knockout rats. Psychopharmacology (Berl) 200(2): 243-254.

Page IH, Rapport MM, Green AA (1948). The crystallization of serotonin. J Lab Clin Med 33(12): 1606.

Page IH (1954). Serotonin (5-hydroxytryptamine). Physiol Rev 34(3): 563-588.

Page IH (1976). The discovery of serotonin. Perspect Biol Med 20(1): 1-8.

Pardridge WM, Fierer G (1990). Transport of tryptophan into brain from the circulating, albumin-bound pool in rats and in rabbits. J Neurochem 54(3): 971-976.

Pardridge WM (1998). Blood-brain barrier carrier-mediated transport and brain metabolism of amino acids. Neurochemical research 23(5): 635-644.

Pletscher A, Shore PA, Brodie BB (1955). Serotonin release as a possible mechanism of reserpine action. Science 122(3165): 374-375.

Rapport MM, Green AA, Page IH (1948a). Serum vasoconstrictor, serotonin; isolation and characterization. J Biol Chem 176(3): 1243-1251.

Rapport MM, Green AA, Page IH (1948b). Crystalline Serotonin. Science 108(2804): 329-330.

Reed RG, Burrington CM (1989). The albumin receptor effect may be due to a surface-induced conformational change in albumin. J Biol Chem 264(17): 9867-9872.

Reilly JG, McTavish SF, Young AH (1997). Rapid depletion of plasma tryptophan: a review of studies and experimental methodology. J Psychopharmacol 11(4): 381-392.

Riedel WJ, Klaassen T, Deutz NE, van Someren A, van Praag HM (1999). Tryptophan depletion in normal volunteers produces selective impairment in memory consolidation. Psychopharmacology (Berl) 141(4): 362-369.

Riedel WJ (2004). Cognitive changes after acute tryptophan depletion: what can they tell us? Psychol Med 34(1): 3-8.

Risch SC, Nemeroff CB (1992). Neurochemical alterations of serotonergic neuronal systems in depression. J Clin Psychiatry 53 Suppl: 3-7. 
Rose WC, Lambert GF, Coon MJ (1954). The amino acid requirements of man. VII. General procedures; the tryptophan requirement. J Biol Chem 211(2): 815-827.

Ruddick JP, Evans AK, Nutt DJ, Lightman SL, Rook GA, Lowry CA (2006). Tryptophan metabolism in the central nervous system: medical implications. Expert Rev Mol Med 8(20): $1-27$.

Ruhe HG, Mason NS, Schene AH (2007). Mood is indirectly related to serotonin, norepinephrine and dopamine levels in humans: a meta-analysis of monoamine depletion studies. Mol Psychiatry 12(4): 331-359.

Rutten K, Lieben C, Smits L, Blokland A (2007). The PDE4 inhibitor rolipram reverses object memory impairment induced by acute tryptophan depletion in the rat. Psychopharmacology (Berl) 192(2): 275-282.

Sambeth A, Blokland A, Harmer CJ, Kilkens TO, Nathan PJ, Porter RJ, Schmitt JA, Scholtissen B, Sobczak S, Young AH, Riedel WJ (2007). Sex differences in the effect of acute tryptophan depletion on declarative episodic memory: a pooled analysis of nine studies. Neurosci Biobehav Rev 31(4): 516-529.

Sambeth A, Riedel W, Tillie D, Blokland A, Postma A, Schmitt J (2009). Memory impairments in humans after acute tryptophan depletion using a novel gelatin-based protein drink. J Psychopharmacol 23(1): 56-64.

Schatzberg AF, Garlow SJ, Nemeroff CB. 2002. Molecular and cellular mechanisms in depression in: Neuropsychopharmacology: the fifth generation of progress. Davis KL, editor. Philadelphia: Lippincott Williams \& Wilkins.

Shannon NJ, Gunnet JW, Moore KE (1986). A comparison of biochemical indices of 5hydroxytryptaminergic neuronal activity following electrical stimulation of the dorsal raphe nucleus. J Neurochem 47(3): 958-965.

Shore PA, Silver SL, Brodie BB (1955). Interaction of reserpine, serotonin, and lysergic acid diethylamide in brain. Science 122(3163): 284-285.

Sjoerdsma A, Palfreyman MG (1990). History of serotonin and serotinin disorders. Ann N Y Acad Sci 600: 1-7; discussion 7-8.

Smith HG, Lakatos C (1971). Effects of acetylsalicylic acid on serum protein binding and metabolism of tryptophan in man. J Pharm Pharmacol 23(3): 180-189.

Smith KA, Fairburn CG, Cowen PJ (1997). Relapse of depression after rapid depletion of tryptophan. Lancet 349(9056): 915-919.

Smith QR, Momma S, Aoyagi M, Rapoport SI (1987). Kinetics of neutral amino acid transport across the blood-brain barrier. J Neurochem 49(5): 1651-1658.

Smith QR (2000). Transport of glutamate and other amino acids at the blood-brain barrier. J Nutr 130(4S Suppl): 1016S-1122S.

Smith SA, Pogson CI (1980). The metabolism of L-tryptophan by isolated rat liver cells. Effect of albumin binding and amino acid competition on oxidatin of tryptophan by tryptophan 2,3dioxygenase. Biochem J 186(3): 977-896.

Stancampiano R, Melis F, Sarais L, Cocco S, Cugusi C, Fadda F (1997). Acute administration of a tryptophan-free amino acid mixture decreases 5 -HT release in rat hippocampus in vivo. Am J Physiol 272(3 Pt 2): R991-994.

Steinbusch HW, Verhofstad AA, Joosten HW (1978). Localization of serotonin in the central nervous system by immunohistochemistry: description of a specific and sensitive technique and some applications. Neuroscience 3(9): 811-819.

Steinbusch HW (1981). Distribution of serotonin-immunoreactivity in the central nervous system of the rat-cell bodies and terminals. Neuroscience 6(4): 557-618.

Stone TW, Darlington LG (2002). Endogenous kynurenines as targets for drug discovery and development. Nat Rev Drug Discov 1(8): 609-620.

Tagliamonte A, Biggio G, Vargiu L, Gessa GL (1973). Free tryptophan in serum controls brain tryptophan level and serotonin synthesis. Life Sci II 12(6): 277-287.

Teff KL, Young SN, Blundell JE (1989). The effect of protein or carbohydrate breakfasts on subsequent plasma amino acid levels, satiety and nutrient selection in normal males. Pharmacology, biochemistry, and behavior 34(4): 829-837. 
Trulson ME (1985). Dietary tryptophan does not alter the function of brain serotonin neurons. Life Sci 37(11): 1067-1072.

Turner EH, Loftis JM, Blackwell AD (2006). Serotonin a la carte: supplementation with the serotonin precursor 5-hydroxytryptophan. Pharmacol Ther 109(3): 325-338.

Twarog BM, Page IH (1953). Serotonin content of some mammalian tissues and urine and a method for its determination. Am J Physiol 175(1): 157-161.

Van der Does AJ (2001). The mood-lowering effect of tryptophan depletion: possible explanation for discrepant findings. Arch Gen Psychiatry 58(2): 200-202.

van der Plasse G, Meerkerk DT, Lieben CK, Blokland A, Feenstra MG (2007). Lack of evidence for reduced prefrontal cortical serotonin and dopamine efflux after acute tryptophan depletion. Psychopharmacology (Berl) 195(3): 377-385.

van der Staay FJ (2006). Animal models of behavioral dysfunctions: basic concepts and classifications, and an evaluation strategy. Brain Res Rev 52(1): 131-159.

van Donkelaar EL, Rutten K, Blokland A, Akkerman S, Steinbusch HW, Prickaerts J (2008). Phosphodiesterase 2 and 5 inhibition attenuates the object memory deficit induced by acute tryptophan depletion. Eur J Pharmacol 600(1-3): 98-104.

van Praag HM, de Haan S (1979). Central serotonin metabolism and frequency of depression. Psychiatry Res 1(3): 219-224.

Whitaker-Azmitia PM (1999). The discovery of serotonin and its role in neuroscience. Neuropsychopharmacology 21(2 Suppl): 2S-8S.

Williams WA, Shoaf SE, Hommer D, Rawlings R, Linnoila M (1999). Effects of acute tryptophan depletion on plasma and cerebrospinal fluid tryptophan and 5-hydroxyindoleacetic acid in normal volunteers. J Neurochem 72(4): 1641-1647.

Young SN, Smith SE, Pihl RO, Ervin FR (1985). Tryptophan depletion causes a rapid lowering of mood in normal males. Psychopharmacology (Berl) 87(2): 173-177.

Young SN, Ervin FR, Pihl RO, Finn P (1989). Biochemical aspects of tryptophan depletion in primates. Psychopharmacology (Berl) 98(4): 508-511.

Young SN (1993). The use of diet and dietary components in the study of factors controlling affect in humans: a review. J Psychiatry Neurosci 18(5): 235-244.

Young VR (1994). Adult amino acid requirements: the case for a major revision in current recommendations. J Nutr 124(8 Suppl): 1517S-1523S. 



\section{Chapter}

\section{Acute tryptophan depletion in healthy C57BL/ 6 mice does not induce reduction of central serotonin levels or affective behavioural changes}

Eva L. van Donkelaar ${ }^{1}$, Arjan Blokland ${ }^{2}$, Cindy K.J. Lieben ${ }^{1}$, Gunter Kenis ${ }^{1}$, Linda Ferrington ${ }^{3}$, Paul A.T. Kelly ${ }^{4}$, Harry W.M. Steinbusch ${ }^{1}$, Jos Prickaerts ${ }^{1}$

${ }^{1}$ Department of Neuroscience, Faculty of Health, Medicine and Life Sciences, School for Mental Health and Neuroscience, Maastricht University, The Netherlands

2 Department of Neuropsychology and Psychopharmacology, Faculty of Psychology and Neuroscience, Maastricht University, The Netherlands

${ }^{3}$ Centre for Cognitive and Neural Systems, University of Edinburgh, United Kingdom

${ }^{4}$ Cerebrovascular Research Laboratory, Centre for Cognitive and Neural Systems, University of Edinburgh, United Kingdom

Neurochemistry International, submitted 



\begin{abstract}
Acute tryptophan depletion (ATD) is extensively used to investigate the implication of serotonin (5-hydroxytryptamine; 5-HT) in the onset of depressive disorders. In rats, it lowers peripheral tryptophan (TRP) and decreases central 5-HT concentrations. We aimed to establish the rat model of ATD in the mouse for potential application as 5-HT challenge tool in genetic mouse models of depression. Pharmacokinetic and behavioural effects of a TRP-free diet were examined in Swiss and C57BL/6 mice. Peripheral amino acids were measured and central TRP and 5-HT concentrations were compared with anxiety and depression-like behaviour in the elevated zero-maze (EZM), forced swimming test (FST) or tail suspension test (TST). While ATD resulted in a $74 \%$ reduction of the plasma ratio TRP to the sum of other large neutral amino acids in Swiss mice $1 \mathrm{~h}$ after administration $(2 \times 10 \mathrm{ml} / \mathrm{kg}, 30 \mathrm{~min}$ interval), there was only a $40 \%$ reduction in C57BL/6 mice. The latter did not show anxiety in the EZM or increased immobility in the FST or TST. A higher dose $(2 \times 20 \mathrm{ml} / \mathrm{kg})$ with a longer interval $(60 \mathrm{~min})$ reduced the ratio with $68 \%$ in $\mathrm{C} 57 \mathrm{BL} / 6$ mice, lowered hippocampal 5-HT turnover and had no functional effect when tested in the EZM and FST. These findings have important implications for the use of ATD in general and in particular for its application in mice. Although in healthy mice no clear central 5-HT or functional effects were observed, further research is indicated using mice with pre-existing 5-HT dysfunction, as they might be more vulnerable to ATD.
\end{abstract}




\section{Introduction}

Although depression is a heterogeneous disorder with complex molecular pathophysiological mechanisms, a dysfunctional serotonergic neurotransmitter system is widely accepted to be one of the main factors underlying the development of depressive symptomatology (Asberg et al., 1976a; Leonard, 2007; Meltzer, 1990). Direct evidence comes from human studies associating depressive symptoms with abnormal physiological parameters related to the serotonergic system (Risch and Nemeroff, 1992). Depressed patients often display disturbances in 5-HT metabolism (van Praag and de Haan, 1979) as indicated by decreased peripheral levels of tryptophan (TRP; Cowen et al., 1989), the dietary precursor of serotonin (5-hydroxytryptamine, 5-HT), and diminished levels of 5-hydroxyindoleacetic acid (5-HIAA; Asberg et al., 1976b), an inactive 5-HT degradation product. Drugs successfully used in the treatment of depression provide direct evidence for the important role of disrupted function of specific pre- and postsynaptic receptors underlying the impaired 5-HT neurotransmission linked to depressive symptomatology (Naughton et al., 2000). Moreover, variations in genes that regulate the 5-HT system appear to play an important role in the probability of onset and recurrence of depressive episodes and other affective disorders (Caspi et al., 2003; Levinson, 2006). Therefore, the aetiology of depression is thought to be strongly related to specific factors that predispose subjects to a dysfunctional 5-HT system (Booij and Van der Does, 2007; Jans et al., 2007b).

The implication of the 5-HT system and serotonergic vulnerability in the onset of depressive symptomatology is being extensively investigated with tools that challenge serotonergic neurotransmission. Most widely applied, clinically and pre-clinically, is the method of acute tryptophan depletion (ATD; (Bell et al., 2005; Hood et al., 2005; Neumeister, 2003; Young, 1993)). By administering a TRP-free nutritional mixture, the dietary intake of TRP is depleted, thereby reducing the TRP for uptake into the brain and subsequent synthesis into 5-HT (Fadda et al., 2000; Fernstrom, 1983). Brain TRP levels correlate highly with brain 5-HT, due to the rate-limiting enzyme tryptophan hydroxylase (TPH), which catalyzes the conversion of TRP into 5-hydroxytryptophan (5-HTP) and is only half saturated under normal physiological conditions (Boadle-Biber, 1993). Manipulation of dietary TRP intake is not only a useful tool for effectively decreasing brain TRP content and 5-HT synthesis, it also provides a clue to understanding the mechanism behind the regulation of the 5-HT system in general. Since the method does not require the use of neurotoxins, it can be safely and repeatedly used in both human and rodent research and offers the ability to compare preclinical and clinical designs and outcomes (Blokland et al., 2004; Fadda et al., 2000). Likewise, the tool makes it possible to measure both physiological and behavioural effects of decreased 5-HT availability in the brain. 
A large body of preclinical literature provides evidence that ATD in rats significantly depletes the levels of TRP in plasma, thereby reducing 5-HT metabolism, as is reflected upon by the lower TRP and 5-HT levels in rat brain tissue (Biggio et al., 1974; Blokland et al., 2002; Cahir et al., 2007; Jans et al., 2007a; Lieben et al., 2004a; Olivier et al., 2008). In addition, ATD in rats induces profound cognitive deficits (Jans et al., 2008a; Jans et al., 2007a; Jans and Blokland, 2008; Lieben et al., 2004b; Olivier et al., 2008; Rutten et al., 2007; van Donkelaar et al., 2008) and a wide range of other behavioural changes (Blokland et al., 2002; Jans et al., 2008a; Jans et al., 2007a). However, whether the ATD-induced functional changes are directly related to a decrease in 5-HT neuronal activity remains unclear (Moore et al., 2000; van der Plasse et al., 2007). Nevertheless, in human subjects, ATD has been proposed as a potential tool for identifying subjects at risk of a future depressive episode, as moodlowering effects have only been reported in healthy subjects with pre-existing abnormal 5-HT functioning (Booij et al., 2002; Booij et al., 2005; Moreno et al., 2000; Van der Does, 2001). Pre-existing dysfunctional 5-HT systems are currently widely modeled in mice by manipulating genes implicated in the regulation of the 5-HT system, such as mice with different expression of the

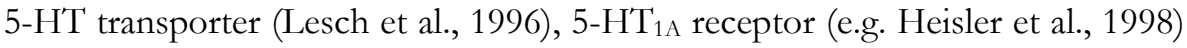
or the tryptophan hydroxylase enzyme (e.g. Zhang et al., 2006).

The introduction of new mouse models of depression is extremely important in order to improve insight into the underlying neurophysiological mechanisms of depressive disorders and for the development of new pharmacological therapies (Cryan and Mombereau, 2004). Therefore, we aimed to establish the rat model of ATD in the mouse for potential future application of the method in transgenic mouse models of serotonergic dysfunction. Triggering pre-existing serotonergic dysfunction by challenging the serotonergic system might reveal specific phenotypical responses that will provide insights into the role of the serotonergic system in depressive symptomatology, in particular, and neuropsychiatric disorders in general.

\section{Experimental procedures}

\section{Animals}

Three-months-old Swiss and C57BL/6 male mice (Charles River, the Netherlands) were used for a total of 5 studies in which the effects of ATD upon peripheral and central TRP levels and brain 5-HT and 5-HIAA concentrations were explored. Some of the studies also included behavioural testing. Swiss mice were used in the first study to obtain initial baseline measures of the pharmacokinetics of the ATD procedure. Subsequent studies were performed on the $\mathrm{C} 57 \mathrm{BL} / 6$ mouse strain as this is the primary background strain of genetically manipulated mice of specific interest in 
depression-related research. All of the studies were carried out at Maastricht University except study four which was conducted at the University of Edinburgh (United Kingdom), using local inbred C57BL/6 mice.

Experimental protocols were reviewed and approved by the local ethical Committee on Animal Experimentation of the Maastricht University and by the University of Edinburgh Ethical Review Process and met Dutch and British governmental guidelines, respectively. Throughout all experiments the principles of laboratory animal care were carefully followed in accordance with the official codes of practice guidelines (Experiments on Animals Act, The Netherlands, revised, 1996; Scientific Procedures Act, United Kingdom, 1986). All efforts were made to minimize the number of mice used and their suffering.

On arrival mice were individually housed on sawdust bedding in standard macrolon cages and maintained on a 12:12-hr light-dark schedule (lights on from 18:00 $\mathrm{h}-06: 00 \mathrm{~h})$, in a temperature-controlled $\left(21 \pm 1{ }^{\circ} \mathrm{C}\right)$ and airconditioned housing room (relative humidity: $70 \pm 10 \%$ ). To facilitate environmental adaptation, mice were not treated or handled until one week after arrival. At all times, background noise in the housing room was provided by a radio. Food (Hopefarms, The Netherlands) and water were available ad libitum throughout the experiments, unless otherwise stated. Animal cages were supplied with paper rolls and tissues to enrich environmental conditions. Clean cages were provided on a weekly basis.

For all studies oral administrations by gavage and blood sampling procedures remained constant following an initial period of habituation to the reversed day-night cycle (1 week) and the general and specific handling (1 week), unless otherwise stated. Habituation also included shaving of the hind paws and the attendant restraint. In addition to familiarization to the oral administrations in general, mice received one administration of the control mixture to avoid gastrointestinal effects interfering at time of the actual experiment. In most studies, animals were repeatedly used for blood sampling and experimental treatment with washout periods of at least 2 days.

To avoid TRP uptake from the available standard chow, food deprivation always preceded experimental treatment. When deprived overnight, food was taken away in the afternoon (6 PM) of the day prior to the experimental day (start of experiment $9 \mathrm{AM}$ ). The exact time and duration of deprivation varied among the different studies. At the time of food removal, animals were transferred to clean cages to eliminate TRP uptake resulting from coprophagy behaviour (Kenagy and Hoyt, 1980). After all animals completed the experimental treatments or behavioural testing, ad libitum food availability was continued. On the day of the experiment, prior to treatment, mice were weighed to calculate the exact injection volume to be administered.

Differences among the studies were mainly based on changes within the dosing regime, including administration volume, amount of additional TRP in the control mixture, number of dosing administrations and the interval between 
them. The time of decapitation, an essential time factor for the measurement of the ATD effects upon physiological parameters in the brain, was explored in more detail in one of the studies. Further, we investigated the role of different stressors that were implicated in the treatment procedures (such as blood sampling, oral administrations by gavage and food deprivation) as potential interfering factors within the 5-HT challenge paradigm.

\section{Acute tryptophan depletion}

Acute reduction of plasma tryptophan levels was accomplished by using a TRPfree protein-carbohydrate nutritional mixture (Gelatine hydrolysate, Solugel ${ }^{\circledR}$; PB Gelatins, Tessenderlo, Belgium). This gelatine-based protein mixture comprises the entire range of amino acids (see Table 1) in the form of peptides and is, therefore, comparable to normal chow (Lieben et al., 2004a). The addition of carbohydrates (Glucodry 210; Amylumgroup, Koog aan de Zaan, The Netherlands) activates the insulin response, thereby enhancing protein synthesis which results in an optimized reduction of plasma TRP levels (Fernstrom and Wurtman, 1972; Fernstrom, 1986; Lieben et al., 2004a; Markus et al., 1998). Since TRP competes at the blood-brain barrier with other large neutral amino acids (LNAAs; leucine [LEU], isoleucine [ILE], phenylalaline [PHE], tyrosine [TYR], valine [VAL]) for the same amino acid L-carrier transport system (Fernstrom and Wurtman, 1972; Fernstrom, 1983), a decrease of the ratio of TRP to the sum of the other LNAAs (TRP/LLNAA) in plasma is normally used as the best predictor of reduced central availability of TRP (Fernstrom, 1979, 1983).

Potassium chloride $(\mathrm{KCl})$ and calcium chloride dihydrate $\left(\mathrm{CaCl}_{2} \cdot 2 \mathrm{H}_{2} \mathrm{O}\right)$ were obtained from Merck (Darmstadt, Germany). To the control mixture, L-Tryptophan (Sigma, Zwijndrecht, The Netherlands) was added, as either $0.28 \%$ or $0.56 \%$ of the total protein amount, depending on the experimental protocol (see below). Mixtures were dissolved in distilled water and freshly prepared in the morning, prior to the start of the experiments.

Animals were orally administered by gavage the TRP-free nutritional mixture (TRP-group) and compared to a group that received the same mixture with additional TRP (TRP+ group). On most occasions, an extra saline group was included to control for specific effects of the nutritional mixture in general

\section{Behavioural testing}

Behavioural testing was conducted in a separate test room, except for the elevated-zero maze test, which took place in the same room as were the animals were housed. At all times, testing took place in the dark phase of the day-night cycle between 10:00 and 16:00 h. Testing was started at specific time-points following ATD treatment initiation. 
Table 1 Composition of the protein-carbohydrate nutritional mixture and determination of the amino acid content of the gelatine-based protein $(\mathrm{g})$.

\begin{tabular}{lll}
\hline Substance & Amount (g) & \\
\hline Protein (Solugel ${ }^{\mathbb{R}}$ ) in $100 \mathrm{ml}$ water & 100 & \\
Alanine & & 8.4 \\
Arginine & & 7.7 \\
Aspartic Acid & & 4.5 \\
Cysteine & & 0.0 \\
Glutamic Acid & & 10.0 \\
Glycine & & 23.3 \\
Histidine & & 0.9 \\
Hydroxylysine & & 1.5 \\
Hydroxyproline & & 12.3 \\
Isoleucine & & 1.2 \\
Leucine & & 2.6 \\
Lysine & & 3.3 \\
Methionine & & 0.9 \\
Phenylalanine & & 1.6 \\
Proline & & 13.7 \\
Serine & & 3.4 \\
Threonine & & 1.9 \\
Tryptophan & & 0.0 \\
Tyrosine & & 0.6 \\
Valine & & 2.2 \\
Carbohydrate (Glucodry 210) in 50 ml water & 50 & \\
KCl in 10 ml water & 0.095 & \\
CaCl 2 2 $\mathrm{H}_{2} \mathrm{O}$ in 10 ml water & 2.318 & \\
L-Tryptophan (TRP+ group) & & \\
L-Tryptophan (TRP- group) & $0.28 / 0.56$ & \\
\hline
\end{tabular}

\section{Elevated zero-maze test}

The elevated zero-maze test (EZM) is a modification of the elevated plus-maze and models anxiety-like behaviour in rodents (Shepherd et al., 1994). The maze consisted of a circular platform (50 cm in diameter), elevated $20 \mathrm{~cm}$ above floor level, with two opposite enclosed parts (50 cm high side walls) and two open parts equally divided along the circular runway $(5 \mathrm{~cm})$. This modified design allows the animal uninterrupted exploration and avoids undefined time spent in the central square as is the case in the traditional elevated plus-maze design (Shepherd et al., 1994). Falls from the open parts were prevented by a $5 \mathrm{~mm}$ high edge. Both the side walls and the maze itself were made of black plastic, transparent for infrared light, and connected via an infrared video camera to a video tracking system (Ethovision Pro, Noldus Wageningen, The Netherlands). For testing, mice were placed in the middle of one of the open parts, facing a closed part, and allowed to explore the maze for a total time of 5 min (study 2) or 6 min (study 3). Total time spent in closed parts and total distance travelled was video tracked under dim light conditions. In between trials, the maze was thoroughly cleaned with water and $70 \%$ alcohol. 


\section{Tail suspension test}

The tail suspension test (Steru et al., 1985), is a measure of behavioural despair after induction of an inescapable haemodynamic stress situation. The tail suspension apparatus consisted of four separate compartments, allowing four animals to be tested simultaneously. The apparatus was positioned in front of a light box to create specific illumination conditions for optimal video tracking. For the test sessions, mice were suspended by their tail for a total time of 6 min. Tail suspension was achieved by wrapping adhesive tape around the midend of the mouse tail and attaching it to a hook inserted in the middle of the roof of the compartment, thereby suspending the mouse head down. The sessions were videotaped from the front with a camera connected to a video tracking system (Ethovision Pro, Noldus, Wageningen, The Netherlands), which allowed automated recording of total immobility time. Settings within Ethovision were adjusted based on manually recorded sessions of four randomly chosen animals (immobility/mobility threshold: $5.7 \%$ ). After 6 min testing, mice were immediately transferred back to their home cage and the apparatus was thoroughly cleaned with water and $70 \%$ alcohol for the following session.

\section{Forced swimming test}

A modified forced swimming test (FST) consisted of exposing the animals to an inescapable water stress, using the total time spent in an immobile position a measure of their level of behavioural despair (Porsolt et al., 1977a; Porsolt et al., 1977b). For the swim sessions, transparent Plexiglas cylinders $(50 \mathrm{~cm}$ in height x $19 \mathrm{~cm}$ in diameter) were filled with warm water $\left(32 \pm 2^{\circ} \mathrm{C}\right)$ up to $20 \mathrm{~cm}$. Water height was specifically chosen to prevent the mice from being able to touch the cylinder bottom with their hind paws or tail (Detke and Lucki, 1996; Lucki, 1997). Four mice were tested in parallel and videotaped from above. Mice were not able to see one another due to grey separation panels placed between the four cylinders. Swim sessions were initiated by transferring the mice from their home cages to one of the cylinders (study 3). In study 2, a 15 min pre-test session preceded the 6 min test-session $24 \mathrm{hrs}$ before. After the swim sessions, mice were immediately removed from the water and transferred to a cage filled with paper towels for an initial 2 min recovery. Subsequently, mice were dried with paper towels and returned to their home cages. In between trials, cylinders were cleaned with $70 \%$ alcohol and refilled with clean water.

The cylinders were positioned on top of a light box to create optimal illumination conditions for video tracking. A camera was placed $75 \mathrm{~cm}$ above the light box and connected to a video tracking system (Ethovision Pro, Noldus, Wageningen, The Netherlands), which allowed automated recording of individual immobility times and total distance moved. Settings within Ethovision were adjusted based on manually recorded sessions of four 
randomly chosen animals (immobility/mobility threshold: $10 \%$ and $13 \%$ for study 2 and 3, respectively).

\section{Blood samples and brain tissue}

Blood samples $( \pm 50 \mu$ per sample) were taken by means of saphenous vein puncture, as this method has been proven to be appropriate for repeated sampling in the same animal in a quick and painless manner and without the requirement of anesthesia (Hem et al., 1998). Mice had both hind paws shaved over the lateral saphenous vein, using a small and almost soundless hair cutting system (Kyone, model GT 100, The Netherlands). For shaving and blood sampling, the animals were placed in a thick cotton cloth, in such a way that the hind paws were freely accessible for the particular procedures. Habituation to the specific restraint procedures and hind paw shaving was included in the initial and overall habituation period as mentioned above.

Blood samples were taken prior to treatment for baseline amino acid (AA) determination in plasma. Subsequent blood samplings were carried out at specific time points after treatment, depending on the particular experimental protocol. At time of decapitation, trunk blood was captured which served for final plasma measurement. Brain amino acids and 5-HT and 5-HIAA concentrations were measured in bilaterally dissected prefrontal cortex and hippocampus. Again, exact time-points depended on the specific protocol used and always coincided with final plasma samples for comparison purposes.

\section{Experimental protocols}

\section{Study 1 Pharmacokinetic effects of different ATD dosing regimes in the Swiss mouse}

Swiss mice ( $n=16)$ were used repeatedly to explore the effects of 4 different dosing regimes upon peripheral TRP levels. Animals were food deprived $4 \mathrm{~h}$ prior to experimental treatment. Administrations of the nutritional mixture took place in a volume of 10 or $20 \mathrm{ml} / \mathrm{kg}$, as either one single dose or in a double dose with a 30 min interval (see overview Table 2).

From the total protein amount, the control mixtures contained $0.28 \%$ of additional TRP. Blood samples were taken approximately $30 \mathrm{~min}$ (t-30; baseline) prior to the start of the treatment ( $\mathrm{t} 0)$ and at $1 \mathrm{~h}(\mathrm{t} 60)$ and $2 \mathrm{~h}(\mathrm{t} 120)$ after the first administration. 
Table 2 Experimental group overview study 1

\begin{tabular}{lllllllll}
\hline $\begin{array}{l}\text { Dosing regime } \\
(\mathrm{ml} / \mathrm{kg})\end{array}$ & $\begin{array}{l}\text { saline } \\
(\mathrm{n})\end{array}$ & $\begin{array}{l}\text { TRP+ } \\
(\mathrm{n})\end{array}$ & $\begin{array}{l}\text { TRP- } \\
(\mathrm{n})\end{array}$ & $\begin{array}{l}\mathrm{t}-30 \\
\mathrm{BS} 1\end{array}$ & $\begin{array}{l}\mathrm{t} 0 \\
\mathrm{adm} 1\end{array}$ & $\begin{array}{l}\mathrm{t} 30 \\
\operatorname{adm} 2\end{array}$ & $\begin{array}{l}\mathrm{t} 60 \\
\mathrm{BS} 2\end{array}$ & $\begin{array}{l}\mathrm{t} 120 \\
\mathrm{BS3}\end{array}$ \\
\hline $1 \mathrm{x} 10$ & 5 & 5 & 6 & $\mathrm{x}$ & $\mathrm{x}$ & - & $\mathrm{x}$ & $\mathrm{x}$ \\
$2 \mathrm{x} 10$ & 5 & 5 & 6 & $\mathrm{x}$ & $\mathrm{x}$ & $\mathrm{x}$ & $\mathrm{x}$ & $\mathrm{x}$ \\
$1 \mathrm{x} 20$ & - & - & 16 & $\mathrm{x}$ & $\mathrm{x}$ & - & $\mathrm{x}$ & $\mathrm{x}$ \\
$2 \mathrm{x} 20$ & - & 8 & 8 & $\mathrm{x}$ & $\mathrm{x}$ & $\mathrm{x}$ & $\mathrm{x}$ & $\mathrm{x}$ \\
\hline
\end{tabular}

TRP+: control nutritional mixture with tryptophan; TRP-: experimental nutritional mixture without tryptophan; BS: blood sampling; adm: oral administration by gavage; t: time in min

Study 2 Pharmacokinetic and behavioural effects of a $2 \times 10 \mathrm{ml}$ ATD dosing regime in the C57BL/ 6 mouse

C57BL/ 6 mice $(n=36)$ were used to examine plasma AA concentration and behavioural effects of the $2 \times 10 \mathrm{ml} / \mathrm{kg}$ dosing regime with a 30 min interval between administrations and $0.28 \%$ additional TRP in the control mixture (same as study 1). After the initial habituation period, experimental treatment and blood sampling was carried out over three consecutive days for which sampling time of second blood sample differed on each day. Mice were deprived of food $3 \mathrm{~h}$ prior to experimental treatment and randomly assigned to blood sampling time group ( $\mathrm{n}=12$ per group/day) and treatment condition (saline $n=4$; TRP $+n=4$; TRP- $n=4$ ). Blood samples for plasma AA determination were taken at baseline (t-30) and either at $1 \mathrm{~h}(\mathrm{t} 60$; day 1$), 2 \mathrm{~h}$ (t120; day 2) or $3 \mathrm{~h} \mathrm{(t180;} \mathrm{day} \mathrm{3)} \mathrm{after} \mathrm{the} \mathrm{first} \mathrm{administration.} \mathrm{Additionally,} \mathrm{this}$ ATD dosing regime was tested for its effects upon depression-like (FST and TST) and anxiety-related (EZM) behaviour (see overview Table 3).

Mice were first exposed to the EZM test ( $5 \mathrm{~min} /$ trial), randomly assigned to one of 3 testing days for which each treatment group was equally represented. Subsequently, with a one week interval to minimize stress effects of previous testing, all mice were exposed to the TST ( $6 \mathrm{~min} /$ trial) within the same day. After another week interval, mice underwent a $15 \mathrm{~min}$ pretest session of the FST, followed $24 \mathrm{hrs}$ later by a 6 min test session. At all times, behavioural testing was started $2 \mathrm{~h}(\mathrm{t} 120)$ after the first oral administration.

Study 3 Pharmacokinetic and behavioural effects of $2 \times 15 \mathrm{ml}$ and $2 \times 20 \mathrm{ml}$ ATD dosing regimes in the C57BL/ 6 mouse

C57BL/ 6 mice $(n=39)$ were used to measure the differential effects of $2 \times 15$ $\mathrm{ml} / \mathrm{kg}$ and $2 \times 20 \mathrm{ml} / \mathrm{kg}$ upon peripheral (AAs), central (AAs, 5-HT, 5-hydroxyindoleacetic acid [5-HIAA]) and behavioural parameters. Mice were randomly assigned to one of two dosing groups ( $\mathrm{n}=16$ per group), receiving either TRP+ $(\mathrm{n}=8)$ or TRP- $(\mathrm{n}=8)$, in a volume of 15 or $20 \mathrm{ml} / \mathrm{kg}$. In addition, 7 mice were treated with saline $( \pm 15 \mathrm{ml} / \mathrm{kg})$. Adaptations to the experimental 
protocol were made based on the results of the previous studies in order to intend reaching maximum plasma TRP depletion effects.

Table 3 Experimental group overview study 2

\begin{tabular}{|c|c|c|c|c|c|c|c|c|c|}
\hline Week & Day & Treatment & $\mathrm{n}$ & $\mathrm{t}-30$ & t0 & $\mathrm{t} 30$ & t60 & $\mathrm{t} 120$ & $\mathrm{t} 180$ \\
\hline \multirow[t]{3}{*}{4} & 1 & $\begin{array}{l}\text { saline, } \\
\text { TRP+, TRP- }\end{array}$ & 12 & BS1 & $\mathrm{adm} 1$ & $\mathrm{adm} 2$ & BS2 & & \\
\hline & 2 & $\begin{array}{l}\text { saline, } \\
\text { TRP+, TRP- }\end{array}$ & 12 & BS1 & $\operatorname{adm} 1$ & $\operatorname{adm} 2$ & - & BS2 & \\
\hline & 3 & $\begin{array}{l}\text { saline, } \\
\text { TRP+, TRP- }\end{array}$ & 12 & BS1 & $\operatorname{adm} 1$ & $\operatorname{adm} 2$ & - & - & BS2 \\
\hline \multirow[t]{3}{*}{6} & 1 & $\begin{array}{l}\text { saline, } \\
\text { TRP+, TRP- }\end{array}$ & 12 & - & $\operatorname{adm} 1$ & $\operatorname{adm} 2$ & - & EZM & \\
\hline & 2 & $\begin{array}{l}\text { saline, } \\
\text { TRP+, TRP- }\end{array}$ & 12 & - & $\operatorname{adm} 1$ & $\operatorname{adm} 2$ & - & EZM & \\
\hline & 3 & $\begin{array}{l}\text { saline, } \\
\text { TRP+, TRP- }\end{array}$ & 12 & - & $\operatorname{adm} 1$ & $\operatorname{adm} .2$ & - & EZM & \\
\hline 7 & 1 & $\begin{array}{l}\text { saline, } \\
\text { TRP+, TRP- }\end{array}$ & 36 & - & $\mathrm{adm} 1$ & $\operatorname{adm} 2$ & - & TST & \\
\hline \multirow[t]{2}{*}{8} & 1 & $\begin{array}{l}\text { saline, } \\
\text { TRP+, TRP- }\end{array}$ & 36 & - & $\operatorname{adm} 1$ & $\operatorname{adm} 2$ & - & $\begin{array}{l}\text { FST } \\
\text { pretest }\end{array}$ & \\
\hline & 2 & $\begin{array}{l}\text { saline, } \\
\text { TRP+, TRP- }\end{array}$ & 36 & - & $\operatorname{adm} 1$ & $\operatorname{adm} 2$ & - & $\begin{array}{l}\text { FST } \\
\text { test }\end{array}$ & \\
\hline \multirow[t]{2}{*}{10} & 1 & $\begin{array}{l}\text { saline, } \\
\text { TRP+, TRP- }\end{array}$ & 18 & BS1 & $\operatorname{adm} 1$ & $\operatorname{adm} 2$ & - & BS2 & \\
\hline & 2 & $\begin{array}{l}\text { saline, } \\
\text { TRP+, TRP- }\end{array}$ & 18 & BS1 & $\operatorname{adm} 1$ & $\operatorname{adm} 2$ & - & BS2 & \\
\hline
\end{tabular}

TRP+: control nutritional mixture with tryptophan; TRP-: experimental nutritional mixture without tryptophan; BS: blood sampling; adm: oral administration by gavage; t: time in min; EZM: elevated zero maze; TST: tail suspension test; FST: forced swimming test

Administrations were given twice, however, this time with a 60 min interval compared to a 30 min interval in the previous studies. Moreover, additional TRP in the control mixture was doubled from $0.28 \%$ to $0.56 \%$ of total protein amount. Mice were food deprived overnight, the day preceding experimental treatment. Behavioural effects of the two dosing regimes were tested $2 \mathrm{~h}$ (t120) after the first administration, as in the previous studies. Mice were first exposed to the EZM ( $6 \mathrm{~min} /$ trial), followed two days later by the FST test ( $6 \mathrm{~min} / \mathrm{trial})$, with 2 days separation to minimize stress effects of previous testing and return to baseline from any treatment effects. Behavioural testing (both EZM and FST) and treatment for brain sampling took place over two consecutive days for which the mice were randomized according to dosing regime. Blood samples were not taken until after termination of the behavioural testing period. Treatment with the $2 \times 15$ and $2 \times 20 \mathrm{ml} / \mathrm{kg}$ dosing regimes was then repeated for 
blood sampling (at t-30, t60 and t120) and subsequent decapitation (t120) for brain tissue measurements (see overview Table 4).

Table 4 Experimental group overview study 3

\begin{tabular}{|c|c|c|c|c|c|c|c|}
\hline & $\begin{array}{l}\text { Dosing regime } \\
(\mathrm{ml} / \mathrm{kg})\end{array}$ & Treatment & $\mathrm{n}$ & $\mathrm{t}-30$ & to & t60 & $\mathrm{t} 120$ \\
\hline \multirow{5}{*}{$\begin{array}{l}\text { week } 4 \\
\text { day } 1+2\end{array}$} & $2 \times 15$ & saline & 7 & & & & \\
\hline & & TRP+ & 8 & & & & \\
\hline & & TRP- & 8 & - & $\operatorname{adm} 1$ & $\operatorname{adm} 2$ & EZM \\
\hline & $2 \times 20$ & TRP+ & 8 & & & & \\
\hline & & TRP- & 8 & & & & \\
\hline \multirow{5}{*}{$\begin{array}{l}\text { week } 4 \\
\text { day } 4+5\end{array}$} & $2 \times 15$ & saline & 7 & & & & \\
\hline & & TRP+ & 8 & & & & \\
\hline & & TRP- & 8 & - & $\operatorname{adm} 1$ & $\operatorname{adm} 2$ & FST \\
\hline & $2 \times 20$ & TRP+ & 8 & & & & \\
\hline & & TRP- & 8 & & & & \\
\hline \multirow[t]{5}{*}{ week 5} & $2 \times 15$ & saline & 7 & & & & \\
\hline & & TRP+ & 8 & & & & \\
\hline & & TRP- & 8 & BS1 & $\operatorname{adm} 1$ & $\mathrm{BS} 2+\operatorname{adm} 2$ & $\mathrm{BS} 3+$ decap \\
\hline & $2 \times 20$ & TRP+ & 8 & & & & \\
\hline & & TRP- & 8 & & & & \\
\hline
\end{tabular}

TRP+: control nutritional mixture with tryptophan; TRP-: experimental nutritional mixture without tryptophan; BS: blood sampling; adm: oral administration by gavage; $t$ : time in min; EZM: elevated zero maze; FST: forced swimming test; decap: decapitation

\section{Study 4 Time curve of pharmacokinetic effects of $A T D$}

C57BL/6J mice ( $\mathrm{n}=31$ ) were used to study the effects of the $1 \times 15 \mathrm{ml} / \mathrm{kg}$ dosing regime upon plasma AAs and brain 5-HT and 5-HIAA at different time points after treatment. Treatment and blood sampling was carried out over three consecutive days without a preceding habituation period to experimental procedures. Mice were food deprived overnight and treated with either TRP- or the control mixture (TRP+) which contained $0.56 \%$ of additional TRP. Decapitations were set at $20 \mathrm{~min}(\mathrm{t} 20), 40 \mathrm{~min}(\mathrm{t} 40), 60 \mathrm{~min}(\mathrm{t} 60)$ or $240 \mathrm{~min}$ (t240). All animals were blood sampled for plasma AA measurements prior to treatment (BS1; baseline) and at time of decapitation (BS2/BS3). The group decapitated at $\mathrm{t} 240$ received a double administration dose $(2 \times 15 \mathrm{ml} / \mathrm{kg})$ with a 60 min interval and an extra blood sample (BS2) taken prior to the second administration (see overview Table 5).

\section{Study 5 Interfering effects of application-related stressors upon ATD pharmacokinetics}

C57BL/6 mice $(n=50)$ were used to measure the effects of stressors related to the experimental procedures and possibly affecting the ATD-induced peripheral and central physiological changes. Food deprivation, oral 
administration by gavage and blood sampling were identified as factors that could affect responses to the ATD treatment. Mice received no oral administrations or either saline, TRP+ or TRP- treatment in a single dose of 15 $\mathrm{ml} / \mathrm{kg}$. The group that received no oral treatment was further subdivided in a food deprived group and a group that continued normal food intake with or without a baseline blood sampling prior to a virtual t0. The other treatment conditions that received oral administrations were all food deprived overnight and had a blood sample taken at time of decapitation. Only half of these mice were also blood sampled at baseline ( $\mathrm{t}-30$; see overview Table 6). All decapitations were carried out at $20 \mathrm{~min}$ from t0. No period of habituation to experimental procedures preceded the actual experiment.

Table 5 Experimental group overview study 4

\begin{tabular}{|c|c|c|c|c|c|c|c|}
\hline Treatment & $\mathrm{n}$ & $t-30$ & $\mathrm{t} 0$ & $\mathrm{t} 20$ & $\mathrm{t} 40$ & t60 & $\mathrm{t} 240$ \\
\hline saline & 4 & & & & & & \\
\hline TRP+ & 4 & BS1 & adm1 & $\mathrm{BS} 2+$ & & & \\
\hline TRP- & 4 & & & decap & & & \\
\hline saline & 4 & & & & & & \\
\hline TRP+ & 3 & BS1 & $\operatorname{adm} 1$ & - & $\mathrm{BS} 2+$ & & \\
\hline TRP- & 4 & & & & decap & & \\
\hline saline & 4 & & & & & & \\
\hline TRP+ & 4 & BS1 & $\mathrm{adm} 1$ & - & - & $\mathrm{BS} 2+$ & \\
\hline TRP- & 4 & & & & & decap & \\
\hline saline & 4 & & & & & & \\
\hline TRP+ & 4 & BS1 & $\mathrm{adm} 1$ & - & - & $\mathrm{BS} 2+$ & $\mathrm{BS} 3+$ \\
\hline TRP- & 4 & & & & & $\operatorname{adm} 2$ & decap \\
\hline
\end{tabular}

TRP+: control nutritional mixture with tryptophan; TRP-: experimental nutritional mixture without tryptophan; BS: blood sampling; adm: oral administration by gavage; $t$ : time in min; decap: decapitation

Table 6 Experimental group overview study 5

\begin{tabular}{lllll}
\hline Food condition & $\mathrm{n}$ & Baseline blood sample (t-30) & Treatment $(\mathrm{t} 0)$ & $\mathrm{t} 20$ \\
\hline Food deprived & 5 & - & saline & decap \\
Food deprived & 5 & - & TRP+ & decap \\
Food deprived & 5 & - & TRP- & decap \\
Food deprived & 5 & - & - & decap \\
Food & 5 & - & - & decap \\
Food & 5 & BS1 & - & decap \\
Food deprived & 5 & BS1 & - & decap \\
Food deprived & 5 & BS1 1 & saline & decap \\
Food deprived & 5 & BS1 & TRP+ & decap \\
Food deprived & 5 & BS1 & TRP- & decap \\
\hline
\end{tabular}

TRP+: control nutritional mixture with tryptophan; TRP-: experimental nutritional mixture without tryptophan; BS: blood sampling; t: time in min; decap: decapitation 


\section{Biochemistry}

Sample preparation and analysis were performed as partly described elsewhere (Double et al., 2003; Vogel et al., 2003). In detail, blood samples for AA determination were collected in heparin coated tubes (Microvette ${ }^{\circledR}$ CB 300, Sarstedt, Germany) and kept on ice until centrifuged (for $10 \mathrm{~min}$ at 14,000 x g in a Hettish EBA 12 centrifuge at $4{ }^{\circ} \mathrm{C}$ ) in order to separate approximately 25 $\mu \mathrm{l}$ of plasma. Plasma samples were subsequently stored at $-80^{\circ} \mathrm{C}$ until further analysis.

At time of decapitation, prefrontal cortex and hippocampus were dissected out bilaterally and immediately put on dry ice before storage at $-80^{\circ} \mathrm{C}$. For central amino acid and monoamine determination, the frozen brain structures were transferred into pre-weighed $2.0 \mu \mathrm{l}$ screw-lit cups and exact tissue weight was determined with an analytic balance. Approximately $0.3 \mathrm{~g}$ of glass beads $(1.0 \mathrm{~mm}$ diameter) were added to the cup together with ice-cold $150 \mathrm{mM}$ $\mathrm{H}_{3} \mathrm{PO}_{4}$ and $500 \mu \mathrm{M}$ diethylenetriamine pantaacetic acid (volume $=19 \mathrm{x}$ weight in $\mu \mathrm{l})$. Samples were aerated with Argon to minimize monoamine degradation and homogenized in a mini-bead beater set at high speed, twice for $30 \mathrm{sec}$. After a subsequent $3 \mathrm{~min}$ spin $\left(3000 \times \mathrm{g}\right.$ at $\left.4^{\circ} \mathrm{C}\right)$, the homogenate was transferred back into an Eppendorf vial and centrifuged for 20 min $(35,000 \mathrm{x} \mathrm{g}$ at $4^{\circ} \mathrm{C}$ ). The supernatant was filtered through Millipore (Bedford, MA) Ultrafree-MC filter cups at $9000 \times \mathrm{g}$ for $1-2 \mathrm{~h}$ at $4^{\circ} \mathrm{C}$ after which they were stored at $-80^{\circ} \mathrm{C}$ until further analysis.

Plasma and brain AA concentrations were quantified using a fully automated high-performance liquid chromatography (HPLC) system with fluorescence detection. The concentrations of total plasma and central amino acids were expressed as $\mu \mathrm{mol} / \mathrm{l}$, brain concentrations as $\mathrm{ng} / \mathrm{mg}$. The concentrations of 5-HT and its metabolite 5-HIAA were quantified by HPLC with electrochemical detection.

\section{Statistical analyses}

Mean concentrations of plasma amino acids were determined for each treatment condition and time-point separately. The mean concentrations of all absolute plasma LNAAs were determined and TRP/ LLNAA ratios were calculated accordingly. Changes in the ratio TRP/ $L$ LNAA over time were analyzed by means of two-way analysis of variance (ANOVA) with Treatment as between-subject factor and Time as within-subject variable. Separate oneway ANOVAs or appropriate t-tests were performed to analyze differences between treatment conditions per time-point and the effects upon behaviour. A post hoc LSD test was used to further characterize the treatment effects. Mean central amino acid concentrations, 5-HT, 5-HIAA and the ratio 5-HIAA/5-HT were analyzed for hippocampus and prefrontal cortex separately. 
Extreme values in the biochemical data (absolute concentrations) were calculated by means of Dixon's Extremity test (Dixon, 1959) and excluded from statistical analysis. In all cases the acceptable level of significance was set at $P<0.05$. All data are represented as mean + standard error of the mean (S.E.M.). Analyses were performed using the Statistical Package for Social Science (SPSS) for Windows (Rel.12.0.1.2003, Chicago: SPSS Inc.). GraphPad Prism, version 4.02 for Windows (GraphPad Software, San Diego California USA) was used for preparing the graphs.

\section{Results}

Study 1 Pharmacokinetic effects of different ATD dosing regimes in the Swiss mouse

\section{Plasma ratio $1 \times 10 \mathrm{ml} / \mathrm{kg}$}

When administering the nutritional mixture once in a volume of $10 \mathrm{ml} / \mathrm{kg}$, ATD decreased the ratio TRP $/ \Sigma \mathrm{LNAA}$ over time $[\mathrm{F}(2,24)=11.74 ; P<0.001]$ and an overall treatment effect $[\mathrm{F}(2,12)=6.69 ; P<0.05]$ was found. Post hoc analysis showed that the TRP- condition was significantly different from TRP+ (LSD; $P<0.01)$. Moreover, the decrease over time was different for the treatment groups $[\mathrm{F}(4,24)=7.38 ; P<0.01]$. Separate one-way ANOVAs for each treatment per time-point showed that there were no differences between treatment conditions at baseline $[\mathrm{F}(2,15)=1.21$; n.s. $]$ or 2 hrs after administration $[\mathrm{F}(2,15)=3.26$; n.s. $]$. However, the ratio TRP/ $/ \mathrm{LNAA}$ was significantly different between treatment conditions $1 \mathrm{~h}$ (t60) after the oral administration $[\mathrm{F}(2,15)=10.64 ; P<0.01]$. Post hoc analyses revealed that at this time-point ATD decreased the ratio TRP/ $\Sigma$ LNAA when compared to saline and TRP+. In the TRP+ condition the ratio was significantly higher when compared to saline. Two hours after administration (t120), the ratio TRP/ $/$ LNAA in the TRP- condition was still significantly reduced when compared to baseline (LSD, $P<0.01$ ). In the TRP+ condition the ratio was not different from baseline values, but was significantly decreased when compared to the $1 \mathrm{~h}$ time-point $(P<0.05$; Fig. $1 \mathrm{~A})$.

\section{Plasma ratio $2 \times 10 \mathrm{ml} / \mathrm{kg}$}

ATD with the $2 \times 10 \mathrm{ml} / \mathrm{kg}$ dosing regime decreased the ratio TRP/ $\mathrm{LLNAA}$ over time $[\mathrm{F}(2,24)=20.86 ; P<0.001]$ and an overall treatment effect $[\mathrm{F}(2,12)$ $=32.25 ; P<0.001]$ was found. Post hoc analyses revealed that the TRPcondition was significantly different from both the saline (LSD; $P<0.001)$ and the TRP+ condition (LSD; $P<0.001)$ and the decrease over time was different for the three treatment groups $[\mathrm{F}(4,24)=39.27 ; P<0.001]$. 
Separate one-way ANOVAs for each treatment group per time-point revealed significant differences between the treatment conditions at $1 \mathrm{~h}[\mathrm{~F}(2,15)=$ 77.75; $P<0.001]$ and $2 \mathrm{~h}[\mathrm{~F}(2,15)=34.13 ; P<0.001]$ after the oral administrations. Post hoc analyses showed that at both time points the ratio TRP/ $/$ LNAA was significantly lower after ATD compared to both the saline and the TRP+ condition. No differences between treatment conditions were found at baseline $[\mathrm{F}(2,14)=0.92$; n.s. $]$ and at all times the saline condition did not differ from the TRP+ treatment (Fig. 1B).
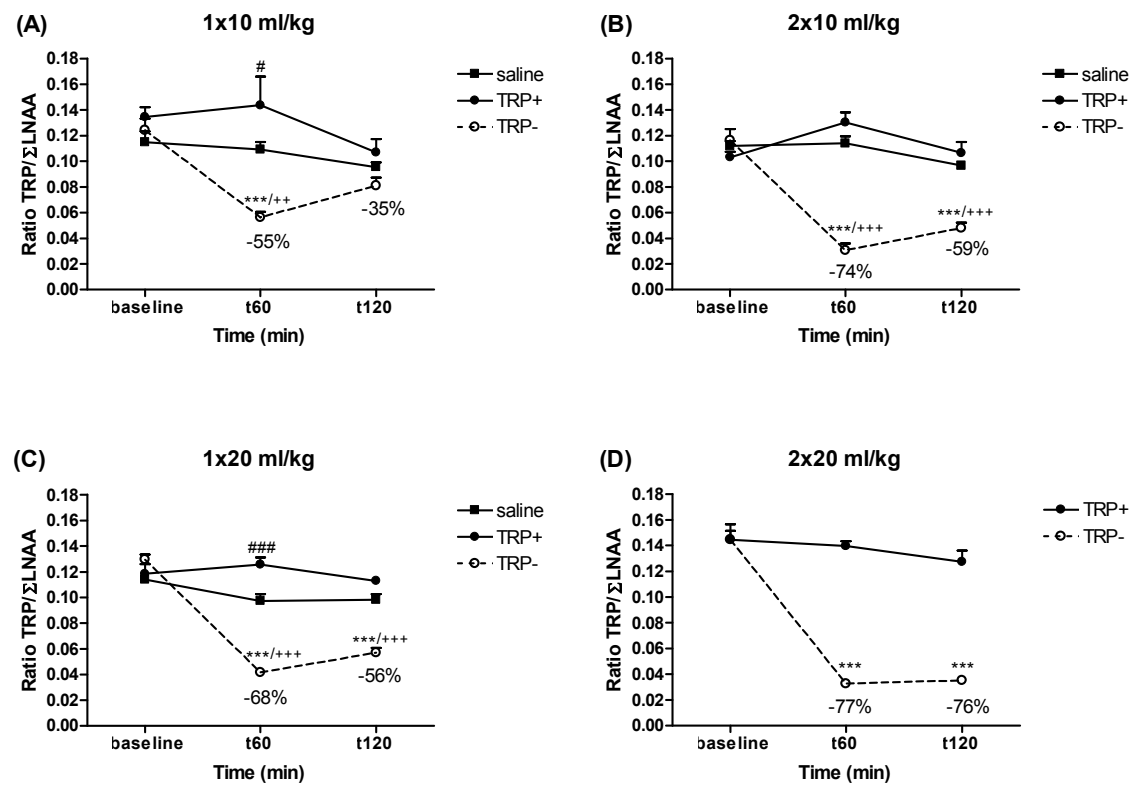

Figure 1 ATD effects of $1 \times 10 \mathrm{ml} / \mathrm{kg}$ (A), $2 \times 10 \mathrm{ml} / \mathrm{kg}$ (B), $1 \times 20 \mathrm{ml} / \mathrm{kg}$ (C) and 2x20 ml/ $\mathrm{kg}$ (D) treatment upon the ratio TRP $/ \Sigma$ LNAA in plasma at different time-points (mean + S.E.M.). $\%$ indicates percentage change from corresponding baseline values. Significant differences between the tryptophan-free experimental mixture (TRP-) and the control mixture with tryptophan $(\mathrm{TRP}+)$ are denoted with $\left(^{*}\right)$, differences between the TRP- and the saline treatment are denoted with $(+)$, and differences between the TRP + and saline treatment are denoted with $(\#) . * /+$ / \# $P<0.05$; **/++/ \#\# $P<0.01$; ***/+++/ \#\#\# $P<0.001$.

\section{Plasma ratio $1 \times 20 \mathrm{ml} / \mathrm{kg}$}

ATD with the $1 \times 20 \mathrm{ml} / \mathrm{kg}$ dosing regime also decreased the ratio TRP $/ \Sigma$ LNAA over time $[\mathrm{F}(2,30)=43.73 ; P<0.001]$ and resulted in an overall treatment effect $[\mathrm{F}(2,15)=56.51 ; P<0.001]$ with a different decrease over time depending on treatment condition $[\mathrm{F}(4,30)=32.31 ; P<0.001]$. Post hoc analyses revealed that the TRP-condition was significantly different from both the saline (LSD; $P<0.001)$ and the TRP+ condition (LSD; $P<0.001)$ and TRP+ 
was different from saline treatment (LSD; $P<0.05)$. Separate one-way ANOVAs for each treatment group per time-point revealed no differences between the treatment conditions at baseline $[\mathrm{F}(2,21)=1.96$; n.s.]. Significant treatment effects were found $1 \mathrm{~h}[\mathrm{~F}(2,21)=170.99 ; P<0.001]$ and $2 \mathrm{~h}[\mathrm{~F}(2,19)$ $=44.61 ; P<0.001]$ after the oral administrations. Post hoc analyses revealed that at the $1 \mathrm{~h}$ time-point the ratio TRP/ $\mathrm{LNAA}$ was significantly lower after ATD when compared to both the saline and the TRP+ treatment. Moreover, after TRP+ treatment, the ratio TRP/ $L \mathrm{LNAA}$ was significantly increased when compared to the saline condition. At the $2 \mathrm{~h}$ time-point, the ratio TRP/ LLNAA was still significantly lower after ATD when compared to saline and TRP+ treatment, however, the difference between TRP+ and saline treatment disappeared at this time-point (Fig. 1C).

\section{Plasma ratio $2 \times 20 \mathrm{ml} / \mathrm{kg}$}

ATD with the $2 \times 20 \mathrm{ml} / \mathrm{kg}$ dosing regime decreased the ratio TRP/ $\mathrm{LLNAA}$ over time $[\mathrm{F}(1,12)=59.01 ; P<0.001]$ and resulted in an overall treatment effect $[\mathrm{F}(1,12)=170.70 ; P<0.001]$ with a different change over time depending on treatment condition $[\mathrm{F}(1,12)=28.08 ; P<0.001]$. Separate independent samples t-tests for the TRP- and TRP+ treatment groups per time-point revealed no differences between the treatment conditions at baseline $[\mathrm{t}(14)=0.00$; n.s.]. A highly significant decrease of the ratio TRP/ $\mathrm{LNNA}$ was found after ATD $1 \mathrm{~h}$ $[\mathrm{t}(13)=25.39, P<0.001]$ and $2 \mathrm{~h}[\mathrm{t}(13)=11.03, P<0.001]$ after administration of the nutritional mixture (Fig. 1D).

\section{Study 2 Pharmacokinetic and behavioural effects of ATD in the C57BL/6 mouse}

\section{Plasma ratio}

ATD in the C57BL/ 6 mouse changed the ratio TRP/ $\Sigma$ LNAA over time $[\mathrm{F}(3$, $21)=10.98, P<0.001]$, however, this pattern did not differ between the treatment conditions $[\mathrm{F}(6,21)=1.24$; n.s.]. An overall treatment effect was found $[\mathrm{F}(2,7)=13.30, P<0.01]$. Post hoc analysis revealed significant differences between the saline and TRP+ and the TRP- and TRP+ treatment condition. Separate one-way ANOVAs per time-point revealed that there were no differences between treatment conditions at baseline $[\mathrm{F}(2,34)=3.157$; n.s. $]$ and $3 \mathrm{~h}$ after the first administration of the $2 \times 10 \mathrm{ml} / \mathrm{kg}$ treatment $[\mathrm{F}(2,10)=$ 0.55 ; n.s.]. Significant treatment effects were found $1 \mathrm{~h}[\mathrm{~F}(2,10)=11.12$, $P<0.01]$ and $2 \mathrm{~h}$ after the first administration $[\mathrm{F}(2,11)=6.76, P<0.05]$. At the $1 \mathrm{~h}$ time-point the ratio TRP/ $\mathrm{LLNAA}$ was significantly lower after ATD as compared to the TRP+ and saline treatment (LSD; $P<0.01)$. The same differences between TRP- and TRP+ and TRP- and saline (LSD; P<0.05) were found $2 \mathrm{~h}$ after the first administration (Fig. 2). 


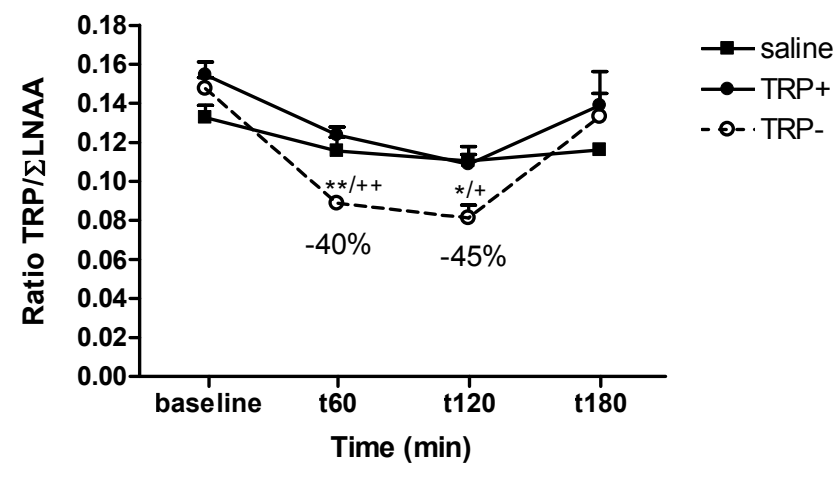

Figure 2 ATD effects of $2 \times 10 \mathrm{ml} / \mathrm{kg}$ treatment upon the ratio TRP $/ \Sigma$ LNAA in plasma at different time-points in the C57BL/ 6 mouse (mean + S.E.M.). \% indicates percentage change from corresponding baseline values. Significant differences between the tryptophan-free experimental mixture (TRP-) and the control mixture with tryptophan (TRP+) are denoted with $\left({ }^{*}\right)$, differences between the TRP- and the saline treatment are denoted with $\left(^{+}\right) .{ }^{* /+} P<0.05$; $* * /++P<0.01$.

\section{Behaviour}

ATD did not produce any anxiety or depression-like behaviour in the C57BL/6 mouse. In the EZM, no differences were found between treatment conditions upon the total time spent in the closed arms $[\mathrm{F}(2,28)=0.56$; n.s.] (Fig. $3 \mathrm{~A})$ or the total distance moved $[\mathrm{F}(2,28)=0.02$; n.s. $]$ (Fig. 3B).

No ATD effects were found in the TST (Fig. 3C) after 6 min upon total immobility time $[F(2,27)=0.085$; n.s.], the amount of time spent hanging immobile during the first $3 \mathrm{~min}[\mathrm{~F}(2,27)=0.16$; n.s. $]$ or during the last $3 \mathrm{~min}$ $[\mathrm{F}(2,27)=1.35$; n.s. $]$.

In the 15 min pre-test, no differences were found between the treatment conditions for the total amount of time spent immobile in the water $[\mathrm{F}(2,35)=$ 0.87 ; n.s.], the mean immobility time during the first 6 min $[\mathrm{F}(2,35)=1.74$; n.s.] or during the last $5 \mathrm{~min}$ of the pre-test $[\mathrm{F}(2,35)=0.35$; n.s.] (Fig. 3D). In the 6 min test 24 hours later (Fig. 3E), ATD did not have an effect either upon the time spent immobile in total $[\mathrm{F}(2,35)=0.55$; n.s.] or during the last $3 \mathrm{~min}$ of the test $[\mathrm{F}(2,35)=1.24$; n.s.]. 
(A)

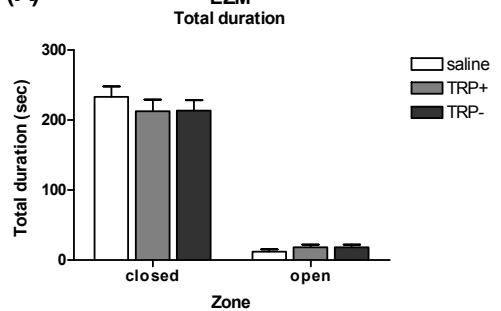

(C)

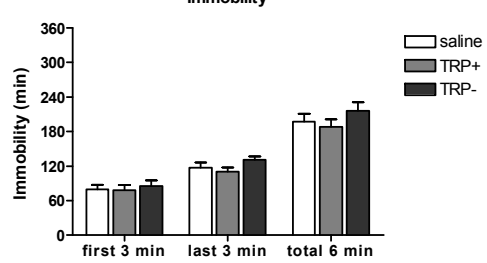

(D)

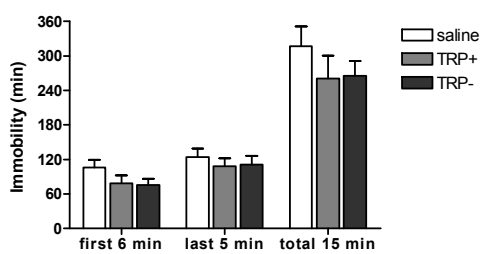

(B)

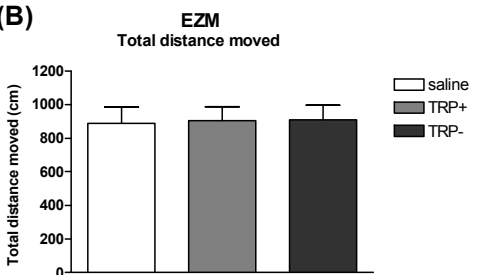

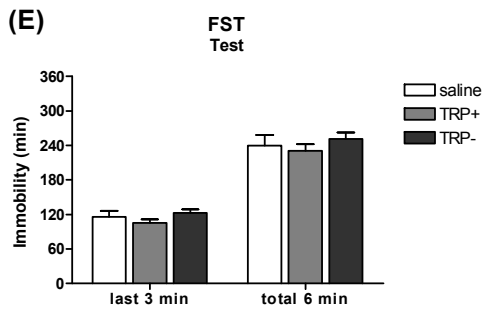

Figure 3 The effects (mean + S.E.M.) of ATD in the C57BL/6 mouse $2 \mathrm{~h}$ after the first administration upon total time spent in the open and closed arms (A) and the total distance moved (B) in the elevated zero maze (EZM), the time spent hanging immobile in the tail suspension test (TST; C) and the immobility time in the 15 min pre-test swim session (D) and the 5 min test session of the forced swimming test (FST; E). No differences were found on any of the behavioural parameters between the tryptophan-free experimental mixture (TRP-) and the control mixture with tryptophan (TRP+).

Study 3 Pharmacokinetic and behavioural effects of two ATD dosing regimes in the C57BL/ 6 mouse

\section{Plasma ratio}

The ratio TRP $/ \Sigma \mathrm{LNAA}$ ratio was decreased over time $[\mathrm{F}(2,60)=229.77$, $P<0.001]$ and an overall treatment effect was found $[\mathrm{F}(4,30)=40.47$, $P<0.001]$. The decrease over time differed for each treatment condition $[\mathrm{F}(8$, $60)=17.05, P<0.001]$. Post hoc analysis showed that the saline treatment was 
different from both the 15 and $20 \mathrm{ml} / \mathrm{kg}$ TRP- treatment (LSD; P<0.001). For both dosing regimes, the TRP- treatment was significantly different from the TRP+ treatment $(P<0.001)$. Separate one-way ANOVAs to reveal treatment effects per time-point showed that the ratio TRP/ $\mathrm{LLNAA}$ was the same for all treatment conditions at baseline $[\mathrm{F}(4,36)=0.63$; n.s.].

Significant differences between treatment conditions were found at $1 \mathrm{~h}[\mathrm{~F}(4$, $37)=64.37, P<0.001]$ and $2 \mathrm{~h}$ after the first treatment administration $[\mathrm{F}(4,37)$ $=45.82, P<0.001]$. From post hoc analyses it can be concluded that at the $1 \mathrm{~h}$ time-point, all the treatment conditions significantly decreased the ratio TRP/ $\Sigma$ LNAA when compared to saline and in both dosing regimes the ratio TRP/ LLNAA was significantly lower after TRP- treatment compared to TRP+ treatment. At the $2 \mathrm{~h}$ time-point the ratio TRP/ $\mathrm{LNAA}$ was slightly higher after TRP+ treatment compared to saline with the $20 \mathrm{ml} / \mathrm{kg}$ administration (Fig. 4A), whereas no differences were found between these two treatment conditions with the $15 \mathrm{ml} / \mathrm{kg}$ dosing regime (Fig. 4B). For both dosing regimes, the ratio TRP/ $L \mathrm{LNAA}$ was still significantly decreased after the TRPtreatment when compared to both the saline and TRP+ condition.
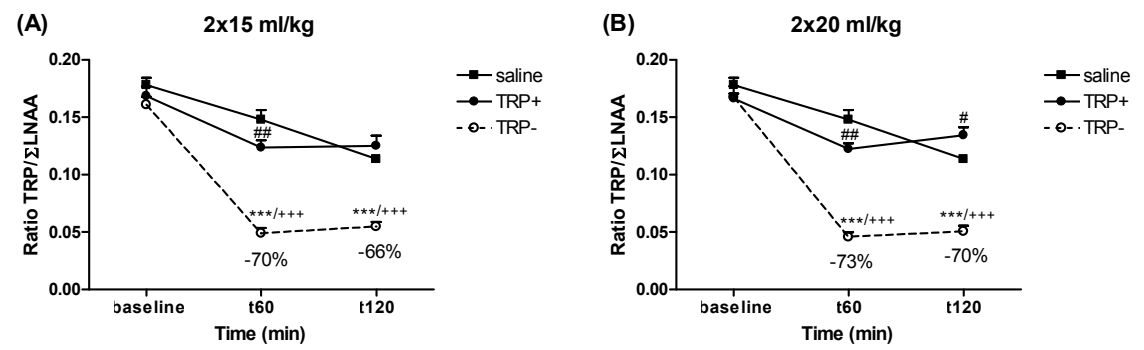

Figure 4 ATD effects of $2 \times 15$ (A) and $2 \times 20 \mathrm{ml} / \mathrm{kg}$ (B) treatment upon the ratio TRP/ $2 \mathrm{LNAA}$ in plasma, $1 \mathrm{~h}$ and $2 \mathrm{~h}$ after the first administration (mean + S.E.M.). Percentages indicate significant changes from corresponding baseline values. Significant differences between the tryptophan-free experimental mixture (TRP-) and the control mixture with tryptophan (TRP+) are denoted with $\left(^{*}\right)$, differences between the TRP- and the saline treatment are denoted with $\left(^{+}\right)$, and differences between the TRP + and saline treatment are denoted with $\left(^{\#}\right) . * /+/ \# P<0.05$; **/++/ \#\# $P<0.01$; ***/+++/ \#\#\# $P<0.001$.

Brain TRP, 5-HT, 5-HLAA and the ratio 5-HLAA/5-HT

In the hippocampus, TRP- treatment did not reduce TRP $[\mathrm{F}(4,37)=1.80$; n.s. $]$ (Fig. 5A), 5-HT $[\mathrm{F}(4,37)=0.98$; n.s. (Fig. 5B) or 5-HIAA concentrations $[\mathrm{F}(4$, $38)=1.83$; n.s.] (Fig. 5C). However, an overall effect was found upon 5-HT turnover (5-HIAA/5-HT) $[\mathrm{F}(4,35)=3.16, P<0.05]$ in this brain area (Fig. 5D). In the prefrontal cortex, an overall treatment effect was also found upon 5-HIAA concentrations $[\mathrm{F}(4,30)=0.61, P<0.05]$ (Fig. 5C), however, no effects of ATD were found upon TRP $[\mathrm{F}(4,36)=0.13$; n.s.] (Fig. 5A) or 5-HT 
concentrations $[\mathrm{F}(4,32)=0.59$; n.s. $($ Fig. 5B) or the 5-HT turnover $[\mathrm{F}(4,30)=$ 0.61; n.s.] (Fig. 5D). Post hoc analyses revealed that in the hippocampus the 5 -HT turnover ratio was significantly reduced after $20 \mathrm{ml} / \mathrm{kg}$ TRP-treatment compared to its TRP+ control treatment. Additionally, the 5-HT turnover after $15 \mathrm{ml} / \mathrm{kg}$ TRP- treatment was significantly lower compared to saline (Fig. 5D). In the frontal cortex, the 5-HIAA levels were significantly reduced after 15 $\mathrm{ml} / \mathrm{kg}$ TRP+ treatment compared to saline (Fig. 5C). Moreover, significantly lower 5-HIAA levels were found after TRP+ treatment with the $15 \mathrm{ml} / \mathrm{kg}$ dosing regime when compared to the $20 \mathrm{ml} / \mathrm{kg}$ dosing regime (Fig. 5C).

(A)

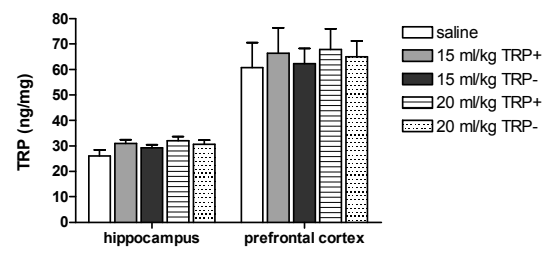

(C)

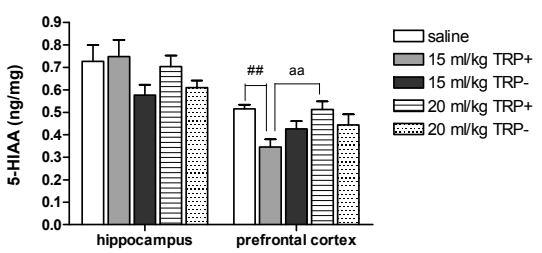

(B)

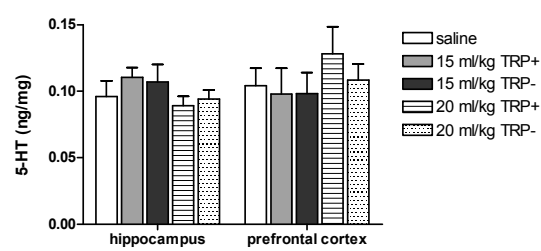

(D)

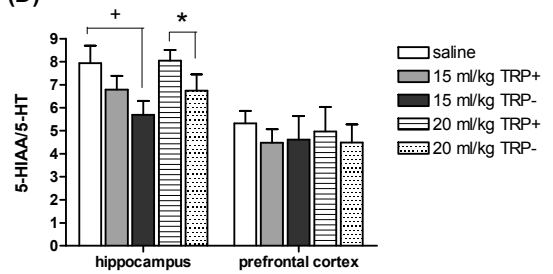

Figure 5 ATD effects of $2 \times 15$ and $2 \times 20 \mathrm{ml} / \mathrm{kg}$ treatment upon central TRP (A), 5-HT (B) and 5-HIAA (C) concentrations and the ratio 5-HIAA/5-HT (D) in hippocampus and frontal cortex, $2 \mathrm{~h}$ after the first administration (mean + S.E.M.). Significant differences between the tryptophan-free experimental mixture (TRP-) and the control mixture with tryptophan (TRP+) are denoted with $(*)$, differences between the TRP- and the saline treatment are denoted with $\left(^{+}\right)$, and differences between the two dosing regimes after TRP+ treatment are denoted with (a). ${ }^{* /+/ \text { a }}$ $P<0.05$; **/++/ aa $P<0.01$.

\section{Behaviour}

In the EZM, no overall treatment effect was found upon the total time spent in the closed arms $[\mathrm{F}(4,37)=0.31$; n.s.] (Fig. 6A). However, the total distance moved after $6 \mathrm{~min}$ (Fig. 6B) was significantly different between the treatment conditions $[\mathrm{F}(4,38)=3.36, P<0.05]$. Post hoc analyses showed that ATD in a volume of $20 \mathrm{ml} / \mathrm{kg}$ reduced the total distance moved of the mice compared to those treated with saline and the ATD mice of the $15 \mathrm{ml} / \mathrm{kg}$ dosing regime. No differences were found, however, between ATD and TRP+ control treatment. 
ATD did not affect the immobility time in the FST (Fig. 6C). No treatment effects were found upon total time spent immobile over the $6 \mathrm{~min}$ swim session $[\mathrm{F}(4,38)=0.86$; n.s. $]$. Analyzing only the first $3 \mathrm{~min}[\mathrm{~F}(4,38)=1.38$; n.s. $]$ or the last 3 min of the swim test $[\mathrm{F}(4,38)=0.42$; n.s.] did not reveal any ATD effects either.

\section{(A)}

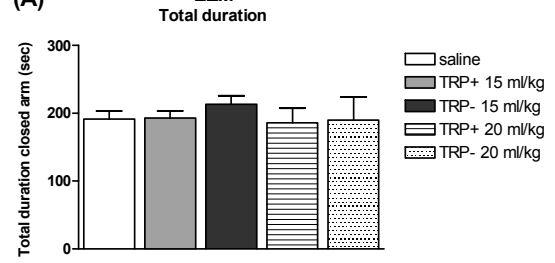

(C)
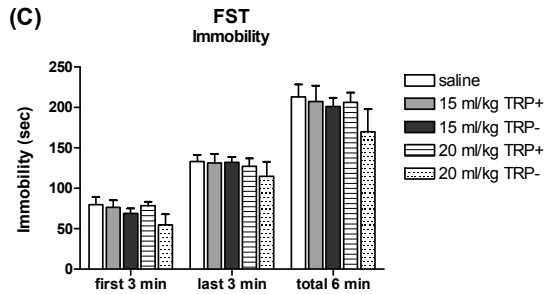

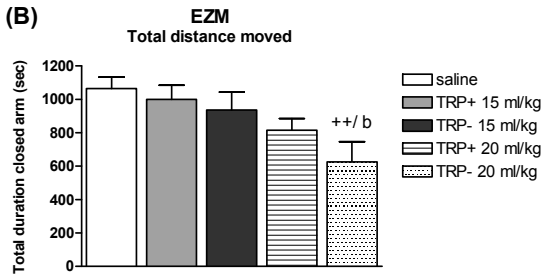

Figure 6 The effects (mean + S.E.M.) of $2 \times 15$ and $2 \times 20 \mathrm{ml} / \mathrm{kg}$ ATD $2 \mathrm{~h}$ after the first administration upon total time spent in the closed arms (A) and the total distance moved (B) in the EZM and the immobility time in a 6 min FST session (C). Significant differences between the TRP- and the saline treatment are denoted with $(+)$ and differences between the two dosing regimes after TRP- treatment are denoted with (b). ${ }^{+/ \mathrm{b}} P<0.05$; $^{++/}$bb $P<0.01$.

Study 4 Time curve of pharmacokinetic effects of $A T D$

\section{Plasma ratio}

ATD changed the plasma ratio TRP $/ \Sigma$ LNAA over time $[\mathrm{F}(4,20)=13.03$, $P<0.001]$ and an overall treatment effect was found $[\mathrm{F}(1,5)=9.44, P<0.05]$. The changes in the ratio TRP/ $/$ LNAA over time did not depend on treatment condition $[\mathrm{F}(4,20)=2.14$; n.s.]. Independent-samples t-tests per time-point revealed that baseline values for ratio TRP/ $\mathrm{LNNA}$ did not differ between the TRP + and TRP- treatment groups $[t(37)=0.144$; n.s.]. Significant decreases in the ratio $\mathrm{TRP} / \Sigma \mathrm{LNAA}$ after $\mathrm{ATD}$ were found after $20 \mathrm{~min}[\mathrm{t}(6)=2.823$; $P<0.05]$ and after $60 \mathrm{~min}[\mathrm{t}(6)=2.924 ; P<0.05]$, not however after $40 \mathrm{~min}[\mathrm{t}(5)$ $=2.277$; n.s.] or $240 \mathrm{~min}[\mathrm{t}(6)=1.042$; n.s.] (Fig. 7). 
Brain 5-HT, 5-HLA A and the ratio 5-HLAA/5-HT

ATD did not change the 5-HT concentration in the hippocampus over time $[F(3,12)=1.13$; n.s. $]$. An overall ATD treatment effect was found $[F(1,4)=$ 5.93, $P<0.05]$, however, independent of time $[F(3,12)=0.08$; n.s.]. Separate $t$-tests per time point did not reveal significant ATD effects at any time point (Fig. 8A). No time effect $[F(3,9)=0.79$; n.s. $]$ or treatment effect $[F(1,3)=$ 1.17; n.s.] was found upon 5-HT concentrations in the prefrontal cortex (Fig. $8 \mathrm{~B})$.

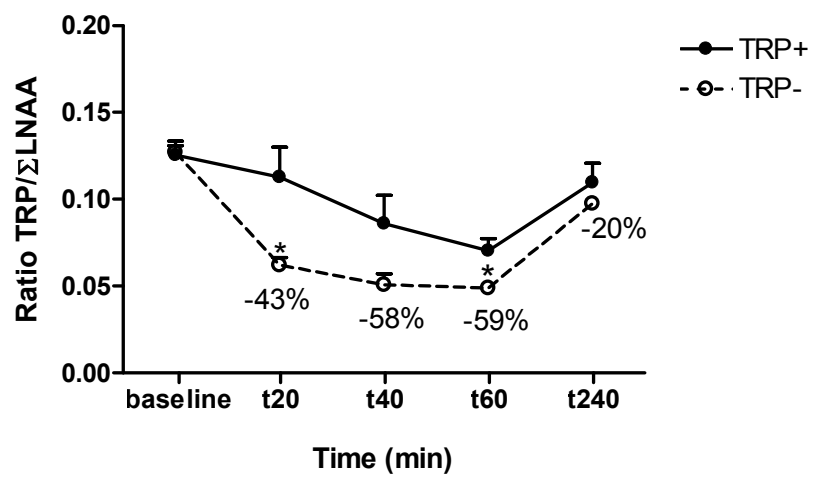

Figure 7 ATD effects of $1 \times 15$ and $2 \times 15 \mathrm{ml} / \mathrm{kg}$ (t240) treatment upon the ratio TRP/ $L$ LNAA in plasma at different time-points (mean + S.E.M.). \% indicates percentage change from corresponding baseline values. $\left(^{*}\right)$ Significant difference between the tryptophan-free experimental mixture (TRP-) and the control mixture with tryptophan (TRP+; $P<0.05)$.

No time effect $[F(3,15)=2.91$; n.s. $]$ or treatment effect $[F(1,5)=0.00$; n.s. $]$ was found upon the 5-HT metabolite, 5-HIAA, in the hippocampus (Fig. 8C). In the prefrontal cortex, ATD changed the concentration 5-HIAA over time $[F(3,12)=3.54, p<0.05$, however, independent of treatment $[F(3,12)=0.45$; n.s.] and without an overall effect of ATD upon 5-HIAA levels. $[F(1,4)=1.97$; n.s.] (Fig. 8D).

The turnover rate of 5-HT (5-HIAA/5-HT) did not change over time in the hippocampus $[F(3,3)=5.98$; n.s.] and no overall treatment effect was found $[F(1,1)=0.28$; n.s.] (Fig. $8 \mathrm{E}$ ). The same was true for the prefrontal cortex, where no time effect $[F(3,9)=0.88$; n.s. $]$ and no overall treatment effect $[F(1$, $3)=0.21$; n.s.] upon the 5-HT turnover rate was found (Fig. 8F). 
Hippocampus

(A)

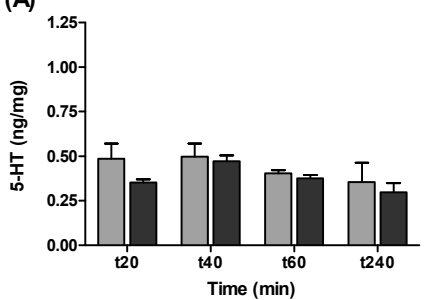

(C)

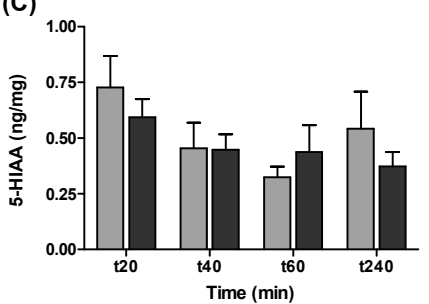

(E)

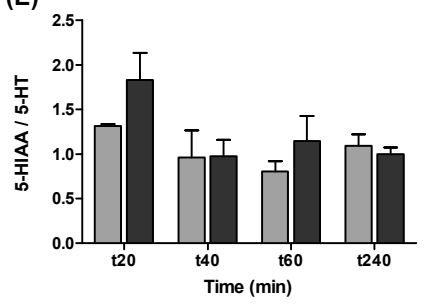

\section{Prefrontal cortex}

(B)

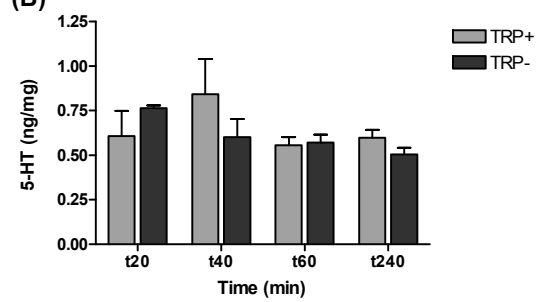

(D)

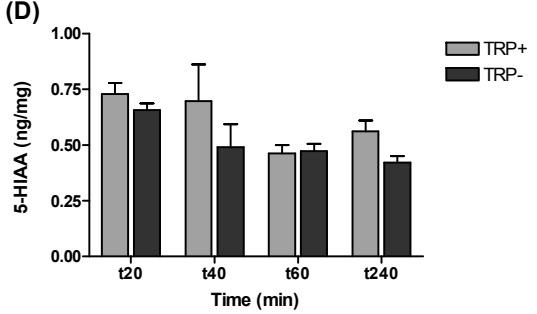

(F)

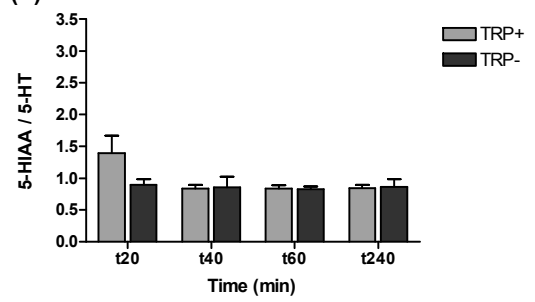

Figure 8 Brain concentrations (mean + S.E.M.) of 5-HT (hippocampus: A; prefrontal cortex: B), 5-HIAA (hippocampus: C; prefrontal cortex: D) and the ratio 5-HIAA/5-HT (hippocampus: E; prefrontal cortex: $\mathrm{F}$ ) at different time points $(\mathrm{t})$ after treatment with $1 \mathrm{x} 15 \mathrm{ml} / \mathrm{kg}(\mathrm{t} 20, \mathrm{t} 40, \mathrm{t} 60)$ or $2 \times 15 \mathrm{ml} / \mathrm{kg}(\mathrm{t} 240)$. No differences were found at any of the time points between the tryptophanfree experimental mixture (TRP-) and the control mixture with tryptophan (TRP+).

\section{Study 5 Interfering effects of experimental stressors upon ATD pharmacokinetics}

One-way ANOVA revealed a significant overall effect $(F(14,72)=3.58$, $P<0.001)$ upon the ratio TRP $/ \Sigma$ LNAA in plasma. LSD post hoc testing allowed for multiple comparisons between the different experimental conditions, effects of which are shown in Fig. 9.

\section{Application effects}

The ratio TRP/ $\Sigma$ LNAA did not significantly decrease at t20 compared to baseline $(P=0.23)$ in the group that was not food deprived overnight or orally treated by gavage. Moreover, no effect was observed of the blood sampling procedure, as no difference was found in the ratio TRP/ $L$ LNAA between the 
group that underwent blood sampling for baseline measurements and the group that did not $(P=0.32)$. At baseline, no differences were found between the food deprived group and the group that had access to food at all times $(P=0.93)$, indicating that food deprivation alone does not directly affect the ratio TRP/ILNAA. In contrast to the group that was not food deprived, a significant decrease of the ratio was found at t20 compared to baseline $(P<0.01)$ in the food deprived group without oral treatment, indicating that food deprivation and blood sampling together, significantly decreased the ratio TRP/ $/$ LNAA. A significant higher TRP/ $/$ LNAA ratio was found at t20 after blood sampling in the saline treated animals compared to the non-treated group $(P<0.05)$, indicating that blood sampling and oral treatment by gavage together significantly increased the ratio TRP/ $\Sigma$ LNAA.

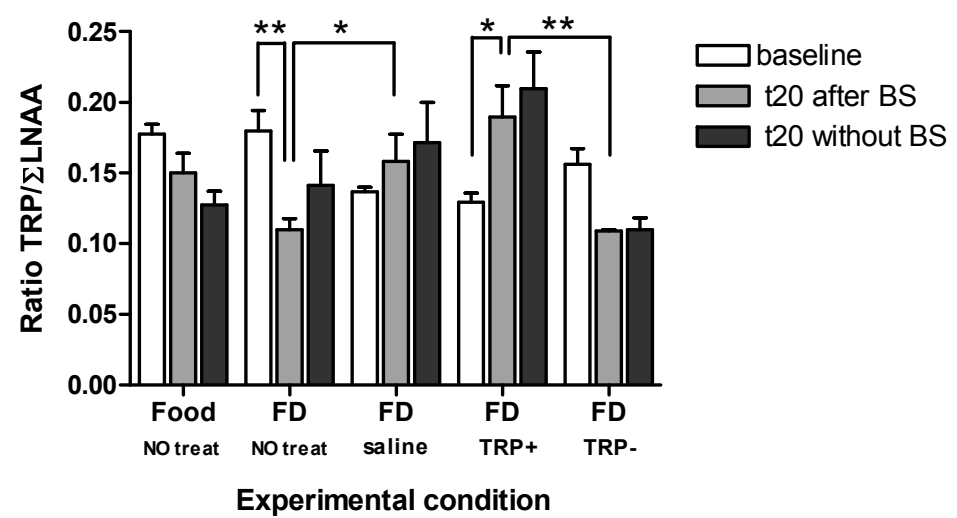

Figure 9 The effects of oral administration by gavage, blood sampling, food deprivation and ATD upon the ratio TRP/ $/$ LNAA in plasma at baseline and $20 \mathrm{~min}$ after administration (mean + S.E.M.). $(*)$ Significant differences in the ratio TRP/ $\Sigma$ LNAA. $* P<0.05, * * P<0.01$. NO treat:: no oral application by gavage; FD: food deprivation; BS: blood sampling; t: time in min. TRP+: control mixture with tryptophan; TRP-: the tryptophan-free experimental mixture (TRP-).

\section{ATD effects}

No differences were found in the ratio TRP/ $\mathrm{LNAA}$ between baseline measurements prior to oral treatment with either saline, TRP+ or TRP-. Saline treatment did not affect the ratio TRP/ LLNAA 20 min after administration compared to baseline $(P=0.38)$ and no differences were found at t 20 between the saline and TRP+ treated animals $(P=0.17)$. A significant increase of the ratio TRP/ $/$ LNAA was found $20 \mathrm{~min}$ after administration of the TRP+ control mixture compared to baseline levels $(P<0.05)$. The ratio TRP $/ \Sigma L N A A$ was significantly lower $20 \mathrm{~min}$ after ATD compared to TRP+ treatment $(P<0.01)$ in the group that received a baseline blood sample. There was only a 
tendency for a decrease in the ratio TRP/ $\Sigma$ LNAA $(P=0.06) 20$ min after ATD compared to TRP+ treatment in the group that did not undergo blood sampling for baseline measurements.

\section{Discussion}

Applying ATD in genetic mouse models of anxiety and depression would allow vulnerable 5 -HT systems to be challenged, paralleling human conditions. The present study, therefore, aimed to introduce the established rat model of ATD to the mouse, specifically the C57BL/ 6 mouse since it is the background mouse strain used for all behavioural experiments in transgenic and knockout research (Gardier and Bourin, 2001). To the best of our knowledge, this is the first study to explore the acute pharmacokinetic and behavioural effects of various dosing regimes of a TRP-free diet in healthy $\mathrm{C} 57 \mathrm{BL} / 6$ mice.

Reducing central 5-HT concentrations by lowering the levels of its dietary precursor TRP in plasma can be achieved by administration of specific TRPfree nutritional mixtures. In comparison to the TRP-free or TRP-low balanced diets and pure amino acid mixtures without TRP, a more advanced technique nowadays is the oral administration of a gelatin-based protein-carbohydrate mixture (Blokland et al., 2004). By adding a specific amount of TRP to the control mixture, suppletion of peripheral TRP levels as often observed with traditional AA mixtures in humans (Fusar-Poli et al., 2006; Van der Does, 2001) is avoided, as is the potential misinterpretation of the ATD effects (Blokland et al., 2004). A double administration of $10 \mathrm{ml} / \mathrm{kg}$ (60 min interval) of this TRPfree mixture in male adult Wistar rats resulted in a considerable depletion of the ratio TRP/ $/ \mathrm{LNAA}$ in plasma after the first administration compared to baseline levels $(71 \%$ and $78 \%$ at 2 and 4 h, respectively; Lieben et al., 2004a). Moreover, with $0.28 \%$ (of the total protein amount) of additional TRP in the control mixture (TRP+), no differences were found between saline treatment and TRP+ treatment (Lieben et al., 2004a).

In the present study, the $2 \times 10 \mathrm{ml} / \mathrm{kg}$ dosing regime as used in rats was initially adapted for application in mice by simply reducing the interval between administrations, thereby speculating upon the generally faster metabolism in mice compared to rats. Thus, optimal peripheral TRP reductions were expected to be within $2 \mathrm{~h}$ after the first administration of the TRP-free mixture. In Swiss mice, the $74 \%$ reduction of the ratio TRP/ $/ \mathrm{LNAA}$ found after $1 \mathrm{~h}$ (study 1 ) was similar to the amount of depletion observed in Wistar rats after $2 \mathrm{~h}$; in contrast, $2 \mathrm{~h}$ after the first administration, the ratio TRP/ $\mathrm{LNNA}$ started to recover, corroborating our speculation of a faster metabolism in mice. However, only a 40\% decrease was found in the C57BL/6 mice with the same dosing regime (study 2), although the C57BL/ 6 mouse recovery after $2 \mathrm{~h}$ was slower which might be indicative of a relatively slower metabolism in these 
mice compared to the Swiss. In addition, slightly higher baseline levels of peripheral TRP can be observed in the C57BL/ 6 mice, which, together with the apparently slower metabolism, could provide an explanation for the lack of considerable TRP depletion and the subsequent unaffected behaviour in these mice.

Although administration with the TRP-free mixture in the C57BL/6 mouse resulted in a significantly lower TRP/ $\Sigma \mathrm{LNAA}$ ratio compared to the other treatments, a reduction of this ratio $2 \mathrm{~h}$ after the first administration was also observed after treatment with saline $(-17 \%)$ and the TRP+ control mixture $(-30 \%)$. This suggests that the control mixture contained too many LNAA compared to the amount of additional TRP $(0.28 \%)$ and thus, was not appropriately balanced to result in an optimal ATD effect in the C57BL/6 mouse strain, in contrast to the Swiss mouse. These discrepancies could be explained by underlying differences in serotonergic neurochemistry between mouse strains which is supported by strain-dependent responses to serotonergic antidepressants (Cervo et al., 2005; Crowley et al., 2005; David et al., 2003; Lucki et al., 2001). In line with this, higher whole-brain 5-HT concentrations have been reported in the C57BL/6 mouse compared to the Swiss (David et al., 2003), which might be attributed to strain differences in the activity of tryptophan hydroxylase (TPH; Cervo et al., 2005), the enzyme that catalyzes the synthesis of TRP into 5 -HT.

A considerable reduction of the ratio TRP/ $\Sigma \mathrm{LNAA}$ was found in the C57BL/6 mice following an increase of the dosing volume to 15 or $20 \mathrm{ml} / \mathrm{kg}$ (study 3). However, doubling the amount of TRP added to the control mixture (from $0.28 \%$ to $0.56 \%$ of total protein amount) did not completely prevent a slight decrease of the ratio after TRP + treatment. Yet, by increasing the interval between administrations (from 30 to $60 \mathrm{~min}$ ) a minor recovery of the ratio was observed after $2 \mathrm{~h}$. This indicates that a single administration of 15 or $20 \mathrm{ml} / \mathrm{kg}$ results in a maximum depletion of 70 and $73 \%$ compared to baseline, respectively. However, by administrating twice over a certain period of time, the duration of the depletion will be prolonged, which has also been reported in rat studies of similar experimental design (Jans et al., 2008b). A similar pattern was also observed in the Swiss mice (study 1) where the maximum depletion $(-77 \%)$ was measured after a double administration of $20 \mathrm{ml} / \mathrm{kg}$.

Despite the considerable reduction of the ratio TRP/ $\mathrm{LNNA}$ in plasma, no changes in central TRP levels or 5-HT concentrations were observed $2 \mathrm{~h}$ after the first administration with either dosing volume (study 3). The conversion of TRP to 5-hydroxytrpytophan by isoforms of the TPH enzyme is the first and rate-limiting step in the biosynthesis of 5-HT. In the brain, this enzyme is only $50 \%$ saturated, thus, the rate at which 5 - HT is synthesised is limited only by substrate, i.e., TRP, availability (Boadle-Biber, 1993). Although most rat studies have reported ATD-induced decreases in central TRP and/or 5-HT (Jans et al., 2008a; Jans et al., 2007a; Lieben et al., 2004a; Olivier et al., 2008), the extent of reduction seems controversial between studies and generally do not parallel the 
acute changes in peripheral TRP levels. Yet, 5-HT seems to be synthesized from TRP by a different isoform of TPH in the periphery (TPH1) compared to the brain (TPH2; Walther and Bader, 2003). This suggests that peripheral 5-HT is not coupled to central 5-HT function and that ATD-induced changes in peripheral TRP and 5-HT might simply not predict central TRP and 5-HT alterations. The results of a study upon the relationship between serotonergic measures in periphery and brain of 7 week-old mice corroborates this concept, as no significant associations were found between blood and brain 5-HT levels or blood TRP levels and brain 5-HT concentration, whereas TRP and 5-HT in the brain were strongly correlated (Pietraszek et al., 1992). However, at this age, the rodent brain is still in development and may therefore regulate amino acid transportation into the brain in a different way (Seta et al., 1972). In addition, cerebral uptake of amino acids is assumed to take longer time in adult mice (Battistin et al., 1971) compared to adult rats (Oldendorf, 1971). Yet, in the present study, no changes in central TRP and 5-HT parameters were found at any of the earlier or later time-points explored, whereas a moderate, though significant, ATD-induced reduction of the ratio TRP/ $/$ LNAA in plasma could already be observed after 20 min (study 4).

Previous studies have revealed that stress might be a critical factor in mediating alterations in the ratio TRP/ $\mathrm{LNAA}$ in plasma and subsequent changes in behavioural performance (Markus et al., 2000a; Markus, 2007; Markus et al., 2000b). Together with other monoamine systems, brain 5-HT is critically involved in the mediation of the central response to stressors and subsequent behavioural adaptation (de Kloet et al., 2005). Acute stressors stimulate the hypothalamic-pituitary-adrenal (HPA) activity, thereby increasing central 5-HT necessary for stress coping (van Praag, 2004). In rats, both acute and repeated exposure to stressful stimuli have been shown to increase glucocorticoid levels and alter 5-HT turnover and release in both the hippocampus and frontal cortex (Chaouloff, 1993). Activation of the sympathetic nervous system increases TRP availability, most probably due to an acute increase in TPH levels as demonstrated after a single exposure of immobilization stress in male Sprague Dawley rats (Chamas et al., 2004). Overnight food deprivation, oral gavage administrations, blood sampling and the immobilization necessary for applying the former can all be considered highly stressful, yet are experimental procedures inevitably implicated in the application of the ATD method. As such, they may interfere with normal brain TRP metabolism and subsequent 5-HT synthesis, thereby acting as confounding factors for the pharmacokinetic and behavioural effects of ATD. While in the present study no isolated effects of the aforementioned experimental stressors were observed, multiple stressors together significantly altered the ratio TRP/ $\mathrm{LLNAA}$ in plasma within $20 \mathrm{~min}$ (study 5). Whereas a significant decrease was found after food deprivation together with blood sampling, oral treatment by gavage after blood sampling and food deprivation increased the ratio TRP/ LLNAA $20 \mathrm{~min}$ after treatment. The latter 
combination represents the standard experimental situation and this suggests that a profound depletion of peripheral TRP is more difficult to establish because of an initial increase in the ratio due to the acute stress of the experimental procedures. Similarly, at a central level the combination of immobilization stress and food deprivation has been reported to increase brain TRP levels in rats considerably more than either of the stressors alone (Curzon et al., 1972). As more 5-HT is needed to be able to cope with the acute stressful situation, brain TRP levels rise to maintain adequate 5-HT concentration.

Although the reduction in hippocampal 5-HIAA levels $2 \mathrm{~h}$ after $2 \times 20 \mathrm{ml} / \mathrm{kg}$ TRP- treatment did not reach statistical significance, the ratio 5-HIAA/5-HT in this brain area was significantly reduced (study 3). Similar patterns of reduced 5-HT turnover in the hippocampus without affecting an analogous effect in prefrontal cortex have also been observed after ATD in rats (Jans et al., 2008a). Changes in central 5-HIAA concentration and the ratio of 5-HIAA to 5-HT have been reported as valid indices of altered 5-HT metabolism (Shannon et al., 1986) and are generally used in animal studies to estimate the rate of 5-HT turnover, which is though to reflect neuronal release. When dietary precursor manipulation is applied, a reduction in 5-HT metabolism is generally attributed to a decrease in precursor availability. The present results, however, do not support this, as no reduction in central TRP or 5-HT levels was observed, at least not in comparison with TRP+ or saline treatment. As in the present study, brain measurements are generally taken at one specific time point which impedes a comparison of the central parameters to baseline values. Therefore, any statement upon ATD-induced absolute changes in central 5-HT or 5-HIAA concentrations and potential underlying mechanisms remain merely speculative.

The ATD-induced decrease in the ratio 5-HIAA/5-HT in the hippocampus can also not explain the reduction in total distance moved in this group as measured during the 6 min exploration in the EZM (study 3). Total distance quantifies overall locomotion (Crawley and Paylor, 1997) and could be used as preliminary indicator of anxiety. A significant decrease in overall locomotion was observed in the mice treated with the TRP-free mixture in the highest volume $(20 \mathrm{ml} / \mathrm{kg})$ compared to both saline and the TRP-treated mice in the lower volume $(15 \mathrm{ml} / \mathrm{kg})$. However, no difference was observed between the TRP+ and TRP- treated animals and no higher anxiety pattern could be revealed from the differences between the groups on the total time spent in the closed parts of the maze. It may therefore be speculated that the reduction in total distance moved is the result of the high administration volume interfering with behavioural performance in which the saline administration would appear to be less invasive than the high caloric mixture of proteins and carbohydrates. As $20 \mathrm{ml} / \mathrm{kg}$ is the maximum volume that may be orally administered to mice (Hull, 1995), twice this volume, albeit with a certain interval, is most likely to compromise their performance on behavioural tasks. 
Although the lack of affective behavioural changes in the present study may reflect the unaltered 5-HT concentrations in the hippocampus and prefrontal cortex, ATD-induced increased anxiety and depressive-like behaviour have not been straightforwardly reported in rats after significant central 5-HT reduction (Brown et al., 1998; Jans et al., 2008a; Lieben et al., 2004b). Only one study reported an increase in mean immobility time in rats after a moderate reduction $(-34 \%)$ of peripheral TRP levels which correlated with a significant decrease of TRP in both the frontal cortex (-31\%) and the hippocampus (39\%) (Blokland et al., 2002). Yet, these reductions in TRP levels were induced by a pure TRPfree amino acid mixture and FST assessment took place $5 \mathrm{~h}$ after ingestion of the mixture with mean immobility times calculated from assessments over 3 consecutive days. Methodological differences of this kind impede outcome comparisons between studies of the same species and rule out further extrapolation to other species. An important observation is that mood-lowering effects after ATD are only reported by healthy subjects with pre-existing abnormal functioning of the 5-HT system (Booij et al., 2002; Booij et al., 2005; Moreno et al., 2000; Van der Does, 2001). ATD-induced anxiety and depressive-like behaviour may therefore only become apparent if the challenge is applied in an already vulnerable system rather than in "healthy" mice or rats.

In conclusion, ATD appears to be a useful tool to lower peripheral TRP concentrations in mice in a manner comparable to that seen in rats. However, to translate the method from one species to the other, fundamental changes are required which appear to go beyond simply reducing administration volumes. In mice, oral administration by gavage with the TRP-free protein-carbohydrate mixture succeeded in reducing peripheral TRP levels significantly to levels comparable to those normally observed in rats after ATD with the same nutritional mixture. Yet, the extent of TRP depletion seems to depend greatly upon the specificity of the dosing volumes and the time frame of the repeated administrations. Moreover, extreme differences were found in the acute pharmacokinetic effects of the nutritional mixture between the Swiss and C57BL/6 mouse strain, due to an apparently slower metabolism in general and higher baseline peripheral TRP levels in particular in the latter. Surprisingly, in contrast to the central ATD effects in rats, the significant reduction of peripheral TRP in the C57BL/6 mouse did not parallel changes in brain TRP concentrations and did not seem to affect 5-HT synthesis or metabolism. This might explain the lack of ATD-induced anxiety and depression-like behaviour, although both 5-HT synthesis reduction and affective behavioural changes have also been reported controversially after ATD in rats. The present results suggest that the various experimental procedures related to ATD application possibly interfere as confounding stressors with the expected decrease in central TRP and 5-HT metabolism after ATD under normal physiological conditions in the mouse. The results of the present studies have important implications for the use of the ATD method in general and in particular for its 
application in mice. More research is needed in this respect, using mice with pre-existing 5-HT dysfunction, as they might be more vulnerable to ATD.

\section{Acknowledgements}

The authors would like to thank Ayhan Şık for his excellent technical assistance and Prof. David A. Hopkins for proofreading the manuscript.

\section{References}

Asberg M, Thoren P, Traskman L, Bertilsson L, Ringberger V (1976a). "Serotonin depression"- a biochemical subgroup within the affective disorders? Science 191(4226): 478-480.

Asberg M, Traskman L, Thoren P (1976b). 5-HIAA in the cerebrospinal fluid. A biochemical suicide predictor? Arch Gen Psychiatry 33(10): 1193-1197.

Battistin L, Grynbaum A, Lajtha A (1971). The uptake of various amino acids by the mouse brain in vivo. Brain Res 29(1): 85-99.

Bell CJ, Hood SD, Nutt DJ (2005). Acute tryptophan depletion. Part II: clinical effects and implications. Aust N Z J Psychiatry 39(7): 565-574.

Biggio G, Fadda F, Fanni P, Tagliamonte A, Gessa GL (1974). Rapid depletion of serum tryptophan, brain tryptophan, serotonin and 5-hydroxyindoleacetic acid by a tryptophan-free diet. Life Sci 14(7): 1321-1329.

Blokland A, Lieben C, Deutz NE (2002). Anxiogenic and depressive-like effects, but no cognitive deficits, after repeated moderate tryptophan depletion in the rat. J Psychopharmacol 16(1): 39-49.

Blokland A, Lieben C, Deutz NEP, Schmitt J (2004). Acute Tryptophan depletion: comparing the effects of an amino acid mixture with a gelatin-based protein in man and rats. Current Topics in Nutraceutical Research 2(3): 1-8.

Boadle-Biber MC (1993). Regulation of serotonin synthesis. Prog Biophys Mol Biol 60(1): 1-15.

Booij L, Van der Does W, Benkelfat C, Bremner JD, Cowen PJ, Fava M, Gillin C, Leyton M, Moore P, Smith KA, Van der Kloot WA (2002). Predictors of mood response to acute tryptophan depletion. A reanalysis. Neuropsychopharmacology 27(5): 852-861.

Booij L, van der Does AJ, Haffmans PM, Spinhoven P, McNally RJ (2005). Acute tryptophan depletion as a model of depressive relapse: behavioural specificity and ethical considerations. Br J Psychiatry 187: 148-154.

Booij L, Van der Does AJ (2007). Cognitive and serotonergic vulnerability to depression: convergent findings. J Abnorm Psychol 116(1): 86-94.

Brown CM, Fletcher PJ, Coscina DV (1998). Acute amino acid loads that deplete brain serotonin fail to alter behavior. Pharmacology, biochemistry, and behavior 59(1): 115-121.

Cahir M, Ardis T, Reynolds GP, Cooper SJ (2007). Acute and chronic tryptophan depletion differentially regulate central $5-\mathrm{HT} 1 \mathrm{~A}$ and $5-\mathrm{HT} 2 \mathrm{~A}$ receptor binding in the rat. Psychopharmacology 190(4): 497-506.

Caspi A, Sugden K, Moffitt TE, Taylor A, Craig IW, Harrington H, McClay J, Mill J, Martin J, Braithwaite A, Poulton R (2003). Influence of life stress on depression: moderation by a polymorphism in the 5-HT'T gene. Science 301(5631): 386-389.

Cervo L, Canetta A, Calcagno E, Burbassi S, Sacchetti G, Caccia S, Fracasso C, Albani D, Forloni G, Invernizzi RW (2005). Genotype-dependent activity of tryptophan hydroxylase-2 determines the response to citalopram in a mouse model of depression. J Neurosci 25(36): 8165-8172. 
Chamas FM, Underwood MD, Arango V, Serova L, Kassir SA, Mann JJ, Sabban EL (2004). Immobilization stress elevates tryptophan hydroxylase mRNA and protein in the rat raphe nuclei. Biol Psychiatry 55(3): 278-283.

Chaouloff F (1993). Physiopharmacological interactions between stress hormones and central serotonergic systems. Brain Res Brain Res Rev 18(1): 1-32.

Cowen PJ, Parry-Billings M, Newsholme EA (1989). Decreased plasma tryptophan levels in major depression. J Affect Disord 16(1): 27-31.

Crawley JN, Paylor R (1997). A proposed test battery and constellations of specific behavioral paradigms to investigate the behavioral phenotypes of transgenic and knockout mice. Horm Behav 31(3): 197-211.

Crowley JJ, Blendy JA, Lucki I (2005). Strain-dependent antidepressant-like effects of citalopram in the mouse tail suspension test. Psychopharmacology (Berl) 183(2): 257-264.

Cryan JF, Mombereau C (2004). In search of a depressed mouse: utility of models for studying depression-related behavior in genetically modified mice. Mol Psychiatry 9(4): 326-357.

Curzon G, Joseph MH, Knott PJ (1972). Effects of immobilization and food deprivation on rat brain tryptophan metabolism. J Neurochem 19(8): 1967-1974.

David DJ, Renard CE, Jolliet P, Hascoet M, Bourin M (2003). Antidepressant-like effects in various mice strains in the forced swimming test. Psychopharmacology (Berl) 166(4): 373382.

de Kloet ER, Joels M, Holsboer F (2005). Stress and the brain: from adaptation to disease. Nat Rev Neurosci 6(6): 463-475.

Detke MJ, Lucki I (1996). Detection of serotonergic and noradrenergic antidepressants in the rat forced swimming test: the effects of water depth. Behav Brain Res 73(1-2): 43-46.

Dixon WJ (1959). Analysis of extreme values. Ann Math Stat 21: 488-506.

Double KL, Halliday GM, Henderson J, Griffiths FM, Heinemann T, Riederer P, Gerlach M (2003). The dopamine receptor agonist lisuride attenuates iron-mediated dopaminergic neurodegeneration. Exp Neurol 184(1): 530-535.

Fadda F, Cocco S, Stancampiano R (2000). A physiological method to selectively decrease brain serotonin release. Brain Res Brain Res Protoc 5(3): 219-222.

Fernstrom JD, Wurtman RJ (1972). Brain serotonin content: physiological regulation by plasma neutral amino acids. Science 178(59): 414-416.

Fernstrom JD (1979). Diet-induced changes in plasma amino acid pattern: effects on the brain uptake of large neutral amino acids, and on brain serotonin synthesis. J Neural Transm Suppl(15): 55-67.

Fernstrom JD (1983). Role of precursor availability in control of monoamine biosynthesis in brain. Physiol Rev 63(2): 484-546.

Fernstrom JD (1986). Acute and chronic effects of protein and carbohydrate ingestion on brain tryptophan levels and serotonin synthesis. Nutr Rev 44 Suppl: 25-36.

Fusar-Poli P, Allen P, McGuire P, Placentino A, Cortesi M, Perez J (2006). Neuroimaging and electrophysiological studies of the effects of acute tryptophan depletion: a systematic review of the literature. Psychopharmacology (Berl) 188(2): 131-143.

Gardier AM, Bourin M (2001). Appropriate use of "knockout" mice as models of depression or models of testing the efficacy of antidepressants. Psychopharmacology (Berl) 153(3): 393394.

Heisler LK, Chu HM, Brennan TJ, Danao JA, Bajwa P, Parsons LH, Tecott LH (1998). Elevated anxiety and antidepressant-like responses in serotonin 5-HT1A receptor mutant mice. Proc Natl Acad Sci U S A 95(25): 15049-15054.

Hem A, Smith AJ, Solberg P (1998). Saphenous vein puncture for blood sampling of the mouse, rat, hamster, gerbil, guinea pig, ferret and mink. Lab Anim 32(4): 364-368.

Hood SD, Bell CJ, Nutt DJ (2005). Acute tryptophan depletion. Part I: rationale and methodology. The Australian and New Zealand journal of psychiatry 39(7): 558-564.

Hull RM (1995). Guideline limit volumes for dosing animals in the preclinical stage of safety evaluation. Toxicology Subcommittee of the Association of the British Pharmaceutical Industry. Hum Exp Toxicol 14(3): 305-307. 
Jans L, Korte-Bouws G, Korte S, Blokland A (2008a). The effects of acute tryptophan depletion on affective behaviour and cognition in Brown Norway and Sprague Dawley rats. J Psychopharmacol.

Jans LA, Lieben CK, Blokland A (2007a). Influence of sex and estrous cycle on the effects of acute tryptophan depletion induced by a gelatin-based mixture in adult Wistar rats. Neuroscience 147(2): 304-317.

Jans LA, Riedel WJ, Markus CR, Blokland A (2007b). Serotonergic vulnerability and depression: assumptions, experimental evidence and implications. Mol Psychiatry 12(6): 522-543.

Jans LA, Blokland A (2008). Influence of chronic mild stress on the behavioural effects of acute tryptophan depletion induced by a gelatin-based mixture. Behav Pharmacol 19(7): 706-715.

Jans LA, Lieben CK, Smits LT, Blokland A (2008b). Pharmacokinetics of acute tryptophan depletion using a gelatin-based protein in male and female Wistar rats. Amino Acids.

Kenagy GJ, Hoyt DF (1980). Reingestion of feces in rodents and its daily rhythmicity. Oecologia 44: 403-409.

Leonard BE (2007). Psychopathology of depression. Drugs of today (Barcelona, Spain: 1998) 43(10): 705-716.

Lesch KP, Bengel D, Heils A, Sabol SZ, Greenberg BD, Petri S, Benjamin J, Muller CR, Hamer DH, Murphy DL (1996). Association of anxiety-related traits with a polymorphism in the serotonin transporter gene regulatory region. Science 274(5292): 1527-1531.

Levinson DF (2006). The genetics of depression: a review. Biol Psychiatry 60(2): 84-92.

Lieben CK, Blokland A, Westerink B, Deutz NE (2004a). Acute tryptophan and serotonin depletion using an optimized tryptophan-free protein-carbohydrate mixture in the adult rat. Neurochem Int 44(1): 9-16.

Lieben CK, van Oorsouw K, Deutz NE, Blokland A (2004b). Acute tryptophan depletion induced by a gelatin-based mixture impairs object memory but not affective behavior and spatial learning in the rat. Behav Brain Res 151(1-2): 53-64.

Lucki I (1997). The forced swimming test as a model for core and component behavioral effects of antidepressant drugs. Behav Pharmacol 8(6-7): 523-532.

Lucki I, Dalvi A, Mayorga AJ (2001). Sensitivity to the effects of pharmacologically selective antidepressants in different strains of mice. Psychopharmacology (Berl) 155(3): 315-322.

Markus CR, Panhuysen G, Tuiten A, Koppeschaar H, Fekkes D, Peters ML (1998). Does carbohydrate-rich, protein-poor food prevent a deterioration of mood and cognitive performance of stress-prone subjects when subjected to a stressful task? Appetite 31(1): 4965.

Markus CR, Olivier B, Panhuysen GE, Van Der Gugten J, Alles MS, Tuiten A, Westenberg HG, Fekkes D, Koppeschaar HF, de Haan EE (2000a). The bovine protein alpha-lactalbumin increases the plasma ratio of tryptophan to the other large neutral amino acids, and in vulnerable subjects raises brain serotonin activity, reduces cortisol concentration, and improves mood under stress. Am J Clin Nutr 71(6): 1536-1544.

Markus CR (2007). Effects of carbohydrates on brain tryptophan availability and stress performance. Biol Psychol 76(1-2): 83-90.

Markus R, Panhuysen G, Tuiten A, Koppeschaar H (2000b). Effects of food on cortisol and mood in vulnerable subjects under controllable and uncontrollable stress. Physiol Behav 70(3-4): 333-342.

Meltzer HY (1990). Role of serotonin in depression. Ann N Y Acad Sci 600: 486-499; discussion 499-500.

Moore P, Landolt HP, Seifritz E, Clark C, Bhatti T, Kelsoe J, Rapaport M, Gillin JC (2000). Clinical and physiological consequences of rapid tryptophan depletion. Neuropsychopharmacology 23(6): 601-622.

Moreno FA, Heninger GR, McGahuey CA, Delgado PL (2000). Tryptophan depletion and risk of depression relapse: a prospective study of tryptophan depletion as a potential predictor of depressive episodes. Biol Psychiatry 48(4): 327-329.

Naughton M, Mulrooney JB, Leonard BE (2000). A review of the role of serotonin receptors in psychiatric disorders. Hum Psychopharmacol 15(6): 397-415. 
Neumeister A (2003). Tryptophan depletion, serotonin, and depression: where do we stand? Psychopharmacol Bull 37(4): 99-115.

Oldendorf WH (1971). Brain uptake of radiolabeled amino acids, amines, and hexoses after arterial injection. Am J Physiol 221(6): 1629-1639.

Olivier JD, Jans LA, Korte-Bouws GA, Korte SM, Deen PM, Cools AR, Ellenbroek BA, Blokland A (2008). Acute tryptophan depletion dose dependently impairs object memory in serotonin transporter knockout rats. Psychopharmacology (Berl) 200(2): 243-254.

Pietraszek MH, Takada Y, Yan D, Urano T, Serizawa K, Takada A (1992). Relationship between serotonergic measures in periphery and the brain of mouse. Life Sci 51(1): 75-82.

Porsolt RD, Bertin A, Jalfre M (1977a). Behavioral despair in mice: a primary screening test for antidepressants. Arch Int Pharmacodyn Ther 229(2): 327-336.

Porsolt RD, Le Pichon M, Jalfre M (1977b). Depression: a new animal model sensitive to antidepressant treatments. Nature 266(5604): 730-732.

Risch SC, Nemeroff CB (1992). Neurochemical alterations of serotonergic neuronal systems in depression. J Clin Psychiatry 53 Suppl: 3-7.

Rutten K, Lieben C, Smits L, Blokland A (2007). The PDE4 inhibitor rolipram reverses object memory impairment induced by acute tryptophan depletion in the rat. Psychopharmacology (Berl) 192(2): 275-282.

Seta K, Sershen H, Lajtha A (1972). Cerebral amino acid uptake in vivo in newborn mice. Brain Res 47(2): 415-425.

Shannon NJ, Gunnet JW, Moore KE (1986). A comparison of biochemical indices of 5hydroxytryptaminergic neuronal activity following electrical stimulation of the dorsal raphe nucleus. J Neurochem 47(3): 958-965.

Shepherd JK, Grewal SS, Fletcher A, Bill DJ, Dourish CT (1994). Behavioural and pharmacological characterisation of the elevated "zero-maze" as an animal model of anxiety. Psychopharmacology (Berl) 116(1): 56-64.

Steru L, Chermat R, Thierry B, Simon P (1985). The tail suspension test: a new method for screening antidepressants in mice. Psychopharmacology (Berl) 85(3): 367-370.

Van der Does AJ (2001). The mood-lowering effect of tryptophan depletion: possible explanation for discrepant findings. Arch Gen Psychiatry 58(2): 200-202.

van der Plasse G, Meerkerk DT, Lieben CK, Blokland A, Feenstra MG (2007). Lack of evidence for reduced prefrontal cortical serotonin and dopamine efflux after acute tryptophan depletion. Psychopharmacology (Berl) 195(3): 377-385.

van Donkelaar EL, Rutten K, Blokland A, Akkerman S, Steinbusch HW, Prickaerts J (2008). Phosphodiesterase 2 and 5 inhibition attenuates the object memory deficit induced by acute tryptophan depletion. Eur J Pharmacol 600(1-3): 98-104.

van Praag HM, de Haan S (1979). Central serotonin metabolism and frequency of depression. Psychiatry Res 1(3): 219-224.

van Praag HM (2004). Can stress cause depression? Prog Neuropsychopharmacol Biol Psychiatry 28(5): 891-907.

Vogel C, Mossner R, Gerlach M, Heinemann T, Murphy DL, Riederer P, Lesch KP, Sommer C (2003). Absence of thermal hyperalgesia in serotonin transporter-deficient mice. Journal of neuroscience 23(2): 708-715.

Walther DJ, Bader M (2003). A unique central tryptophan hydroxylase isoform. Biochem Pharmacol 66(9): 1673-1680.

Young SN (1993). The use of diet and dietary components in the study of factors controlling affect in humans: a review. J Psychiatry Neurosci 18(5): 235-244.

Zhang X, Beaulieu JM, Gainetdinov RR, Caron MG (2006). Functional polymorphisms of the brain serotonin synthesizing enzyme tryptophan hydroxylase-2. Cell Mol Life Sci 63(1): 6-11. 



\section{Chapter}

\section{Acute tryptophan depletion in rats alters the relationship between cerebral blood flow and glucose metabolism independent of central}

serotonin

Eva L. van Donkelaar ${ }^{1}$, Linda Ferrington ${ }^{2}$, Arjan Blokland ${ }^{3}$, Harry W.M. Steinbusch ${ }^{1}$, Jos Prickaerts ${ }^{1}$, Paul A.T. Kelly ${ }^{4}$

${ }^{1}$ Department of Neuroscience, Faculty of Health, Medicine and Life Sciences, School for Mental Health and Neuroscience, Maastricht University, The Netherlands

${ }^{2}$ Centre for Cognitive and Neural Systems, University of Edinburgh, United Kingdom

${ }^{3}$ Department of Neuropsychology and Psychopharmacology, Faculty of Psychology and Neuroscience, Maastricht University, The Netherlands

${ }^{4}$ Cerebrovascular Research Laboratory, Centre for Cognitive and Neural Systems, University of Edinburgh, United Kingdom 



\begin{abstract}
Acute tryptophan depletion (ATD) decreases the serotonin (5-hydroxytryptophan; 5-HT) precursor tryptophan (TRP) in blood and is used both clinically and pre-clinically to investigate the involvement of 5-HT in the development of depressive symptomatology. Depression is associated with both central 5-HT dysfunction and abnormalities in the normal relationship between regional cerebral blood flow $(\mathrm{CBF})$ and glucose metabolism (CMRG). In this study, ATD was applied in Wistar rats to investigate the cerebrovascular effects of acute changes in peripheral TRP. CBF or CMRG were measured $4 \mathrm{~h}$ after ATD initiation by quantitative autoradiographic imaging in 43 brain regions of interest (ROI). In plasma, ATD resulted in a $40 \%$ reduction in the ratio of TRP to the sum of all other large neutral amino acids, but had no measurable effect upon TRP or 5-HT levels in hippocampus or prefrontal cortex. Nevertheless, ATD significantly reduced local CBF in 11 of the 43 brain ROIs, while local CMRG remained unchanged. Global analysis of all 43 ROIs revealed a close correlation between CBF and CMRG within both treatment groups. However, the overall ratio (=slope) after ATD $(\mathrm{m}=1.07)$ was significantly decreased compared to the control group $(\mathrm{m}=1.27)$, indicating a state of relative cerebral oligaemia. Since ATD induced a significant lowering of peripheral TRP, without affecting central TRP or 5-HT concentrations, the decrease in $\mathrm{CBF}$ and global change in flow-metabolism coupling cannot be directly attributed to decreases in brain TRP availability. Nevertheless, these data suggest that cerebrovascular disturbances should be considered as a potential contributory factor in studies of serotonergic dysfunction, including depression, with important implications for imaging studies that use CBF alone as a measure of neuronal function.
\end{abstract}




\section{Introduction}

Many physiological and functional processes are regulated by the serotonergic system, including mood and cognition, and these are most prominently impaired in clinical depression (Leonard, 1997; Maes and Meltzer, 1995). Acute tryptophan depletion (ATD) is a non-toxic challenge that can be used for repeated manipulation of the central serotonergic system in vivo and is currently used, in both preclinical and clinical research, to investigate the involvement of the serotonergic system in the pathogenesis and pathophysiology of mood disorders (Bell et al., 2005; for review see: Fadda et al., 2000b; Hood et al., 2005; Neumeister, 2003). To date, however, the exact mechanism by which ATD exerts its neurophysiological effects, and to what extent changes in 5-HT neuronal activity contribute to the ATD-induced functional and behavioural alterations, is not fully understood.

The procedure generally consists of reducing the concentration of the monoamine serotonin (5-hydroxytryptamine; 5-HT) by temporarily lowering the levels of its dietary precursor tryptophan (TRP) in plasma (Biggio et al., 1974; Gessa et al., 1975). A large body of preclinical literature provides evidence that ATD significantly decreases the levels of TRP in plasma, resulting in substantial reductions of TRP and 5-HT metabolites in the brain (e.g. Biggio et al., 1974; e.g. Blokland et al., 2002; Cahir et al., 2007; Gessa et al., 1974; Jans et al., 2007a; Lieben et al., 2004a; Olivier et al., 2008). However, actual decreases in extracellular 5-HT have only been reported after ATD in combination with blockade of 5-HT reuptake (Bel and Artigas, 1996; Fadda et al., 2000a; Stancampiano et al., 1997). Nevertheless, ATD clearly induces cognitive deficits in rats (Jans et al., 2007a; Lieben et al., 2004b; Rutten et al., 2007; van Donkelaar et al., 2008), whereas, alterations in affective behavioural parameters are somewhat controversial (Blokland et al., 2002; Brown et al., 1998; Lieben et al., 2004b).

For clinical research, ATD challenge has been proposed as a potential tool for predicting subjects at risk of a future depressive episode (Booij et al., 2002; Booij et al., 2005b; Jans et al., 2007b; Moreno et al., 2000a), since moodlowering effects have only been reported in healthy subjects predisposed to serotonergic vulnerability (e.g. Booij et al., 2002; Booij et al., 2003; Klaassen et al., 1999; Neumeister et al., 2002; Neumeister et al., 2006; Smith et al., 1997). Nevertheless, 5-HT metabolism seems substantially lowered after ATD (Carpenter et al., 1998; Moreno et al., 2000b; Williams et al., 1999) and in all sub-populations evenly. This might explain why ATD-induced cognitive deficits have been observed in both healthy and vulnerable subjects (Booij et al., 2005a; Evers et al., 2005; Klaassen et al., 2002; Riedel et al., 1999; Riedel, 2004; Sambeth et al., 2007; Sambeth et al., 2009). Taken together, the implications of preclinical and clinical ATD-induced behavioural effects for depression research do not appear to be straightforward. 
In line with the serotonergic hypothesis of depression (Maes and Meltzer, 1995), ATD-induced behavioural changes are thought to reflect decreased 5-HT neuronal activity in brain regions responsible for the specific behavioural output. Under normal physiological circumstances, neuronal activity is closely related to energy generation which is in turn closely coupled to cerebral blood flow (Kuschinsky, 1991; Sokoloff, 1981a). 5-HT has important vasoactive properties and may thus be involved in both the modulation of metabolic demand and the regulation of substrate delivery (Cohen et al., 1996; Kelly et al., 1988; McBean et al., 1991; Nobler et al., 1999). Interestingly, reduced local cerebral blood flow $(\mathrm{CBF})$ in the anterior cingulate and subgenual prefrontal cortex has been observed in healthy subjects after ATD (Talbot and Cooper, 2006).

Neuroimaging studies of depressed subjects have revealed abnormal CBF and local glucose metabolism (CMRG) in those brain regions implicated in emotional processing and behaviour (Drevets, 2000, 2001; Soares and Mann, 1997). Positron emission tomography (PET) studies of medicated remitted subjects found that ATD-induced cognitive impairment and depressive relapse were associated with decreased metabolism in the orbital frontal, cingulate and parietal cortices and caudate nucleus (Bremner et al., 1997; Smith et al., 1999). In contrast, in unmedicated remitted subjects, increased metabolism was found in similar regions (Neumeister et al., 2004). Additionally, there is some evidence that changes in the relationship between CMRG and CBF are found in depression (Drevets, 2000) and a close correlation has been suggested to exist between the degree of uncoupling and the severity of the depressive symptoms (Dunn et al., 2005). Whether this might also be induced by ATD, is not known

The purpose of the present study was, to measure CMRG and CBF in parallel groups of rats following ATD in order to examine both the neural response and cerebrovascular effects of the treatment. It was hypothesised that by lowering peripheral levels of the 5-HT precursor TRP, the flow-metabolism relationship might be affected by a reduction in serotonergic vasoconstrictor tone. Such an effect might call into question the validity of using CBF as an index of neuronal activity in human imaging studies of ATD effects in particular, but also perhaps clinical depression in general.

\section{Experimental procedures}

\section{Animals}

A total of 23 male Wistar rats (Charles River, UK) was used in this study, with an average weight of 350 grams at the start of the experiments. Animals were group-housed under standard laboratory conditions in a temperature controlled colony room $\left(21 \pm 2^{\circ} \mathrm{C}\right)$ and maintained on a 12:12-hr light-dark schedule 
(lights on from 07:00 h - 19:00 h). Food and water were available ad libitum until the night before the experimental day.

All efforts were made to minimize the number of animals used and their suffering. Animal procedures were performed with local ethical approval from the University of Edinburgh Ethical Review Process, and in accordance with the United Kingdom Animals (Scientific Procedures) Act 1986 and associated national and international guidelines.

\section{ATD treatment}

Acute reduction of plasma tryptophan levels was achieved by oral application with a TRP-free protein-carbohydrate nutritional mixture. This gelatine-based protein nutritional mixture (Gelatine hydrolysate, Solugel ${ }^{\circledR}$; PB Gelatins, Tessenderlo, Belgium) comprises the entire range of amino acids (see Table 1 for amino acid composition) in the form of peptides, as found in normal food (Lieben et al., 2004a). The addition of carbohydrates (Glucodry 210; Amylumgroup, Koog aan de Zaan, The Netherlands) activates the insulin response, and the resulting increase in protein synthesis promotes an optimized reduction in plasma TRP levels (Fernstrom, 1986; Lieben et al., 2004a; Markus et al., 1998). Potassium chloride $(\mathrm{KCl})$ and calcium chloride dihydrate $\left(\mathrm{CaCl}_{2} \cdot 2 \mathrm{H}_{2} \mathrm{O}\right)$ were obtained from Merck (Darmstadt, Germany). For the control group, L-tryptophan (Sigma, Zwijndrecht, The Netherlands) was added in an amount equivalent to $0.28 \%$ of total protein content, as adapted from Lieben et al. (2004a). Mixtures were dissolved in distilled water and freshly prepared on the day of the experiment. The availability of TRP for synthesis into 5-HT in the brain is also heavily influenced by the intake of other large neutral amino acids (LNAA; leucine [LEU], isoleucine [LE], tyrosine [TYR], valine [VAL] and phenylalaline [PHE]), since they all compete at the bloodbrain barrier for the same amino acid L-carrier transport system (Fernstrom and Wurtman, 1972; Fernstrom, 1983). Therefore, a decrease in the ratio of TRP to the sum of other LNAAs in plasma (TRP/ $L \mathrm{LNAA}$ ) is normally used as the best predictor of reduced central availability of TRP (Fernstrom, 1979, 1983).

Surgical procedures were carried out as described elsewhere (Ferrington et al., 2006; Kelly et al., 1995; Quate et al., 2004). For the measurement of the effects of ATD upon local CBF and CMRG, animals were randomly assigned to one of the treatment groups. One hour following recovery from surgery, animals were orally dosed by gavage, twice with a 90 min interval (Lieben et al., 2004a). Administration consisted of the nutritional mixture, with (TRP+ group) or without TRP (TRP-group), in a volume of $10 \mathrm{ml} / \mathrm{kg}$. Each administration contained $4 \mathrm{~g} / \mathrm{kg}$ bodyweight of Solugel ${ }^{\circledR}$ and $2 \mathrm{~g} / \mathrm{kg}$ Glucodry. The availability of dietary TRP was reduced by removing food from the cages on the night before the experiments. Water was available at all times during the experiment. 
Table 1 Composition of the protein-carbohydrate nutritional mixture and determination of the amino acid content $\left(\mathrm{g} / 100 \mathrm{ml}\right.$ water) of the gelatine based protein $\left(\right.$ Solugel $\left.{ }^{\mathbb{R}}\right)$

\begin{tabular}{|c|c|}
\hline Substance & Amount (g) \\
\hline $\begin{array}{l}\text { Protein }\left(\text { Solugel }{ }^{\circledR}\right) \text { in } 100 \mathrm{ml} \text { water } \\
\text { Alanine } \\
\text { Arginine } \\
\text { Aspartic Acid } \\
\text { Cysteine } \\
\text { Glutamic Acid } \\
\text { Glycine } \\
\text { Histidine } \\
\text { Hydroxylysine } \\
\text { Hydroxyproline } \\
\text { Isoleucine } \\
\text { Leucine } \\
\text { Lysine } \\
\text { Methionine } \\
\text { Phenylalanine } \\
\text { Proline } \\
\text { Serine } \\
\text { Threonine } \\
\text { Tryptophan } \\
\text { Tyrosine } \\
\text { Valine }\end{array}$ & $\begin{array}{l}8.4 \\
7.7 \\
4.5 \\
0.0 \\
10.0 \\
23.3 \\
0.9 \\
1.5 \\
12.3 \\
1.2 \\
2.6 \\
3.3 \\
0.9 \\
1.6 \\
13.7 \\
3.4 \\
1.9 \\
0.0 \\
0.6 \\
2.2\end{array}$ \\
\hline $\begin{array}{l}\text { Carbohydrate (Glucodry } 210 \text { ) in } 50 \mathrm{ml} \text { water } \\
\mathrm{KCL} \text { in } 10 \mathrm{ml} \text { water } \\
\mathrm{CaCl}_{2} \cdot 2 \mathrm{H}_{2} \mathrm{O} \text { in } 10 \mathrm{ml} \text { water } \\
\mathrm{L} \text {-tryptophan (TRP+ group) } \\
\mathrm{L} \text {-tryptophan (TRP- group) }\end{array}$ & $\begin{array}{l}50 \\
0.095 \\
2.318 \\
0.28 \\
0\end{array}$ \\
\hline
\end{tabular}

The median point of integrated specific 2-deoxyglucose in the brain, from which CMRG is derived, is around 10-20 min after an intravenous bolus injection of the tracer (Sokoloff et al., 1977) and CBF studies were timed to coincide with this, as previously described (Ferrington et al., 2006; Kelly et al., 1995; Quate et al., 2004). Other studies using the same nutritional mixture (Solugel $^{\circledR}$ ) and dosing regime (Cahir et al., 2008; Jans et al., 2007a; Jans et al., 2008; Lieben et al., 2004a; van der Plasse et al., 2007) have reported significant decreases in both peripheral and central TRP concentrations in male Wistar rats, approximately $4 \mathrm{~h}$ after the first oral administration of the TRP-free nutritional mixture. Therefore, $\mathrm{CMRG}$ and $\mathrm{CBF}$ measurements were initiated at 220 and $240 \mathrm{~min}$ after the first oral administration by gavage, respectively (see also Fig. 1 for an overview of the different phases of the experiment). Mean arterial blood pressure and rectal temperature were monitored throughout the entire experiment. 


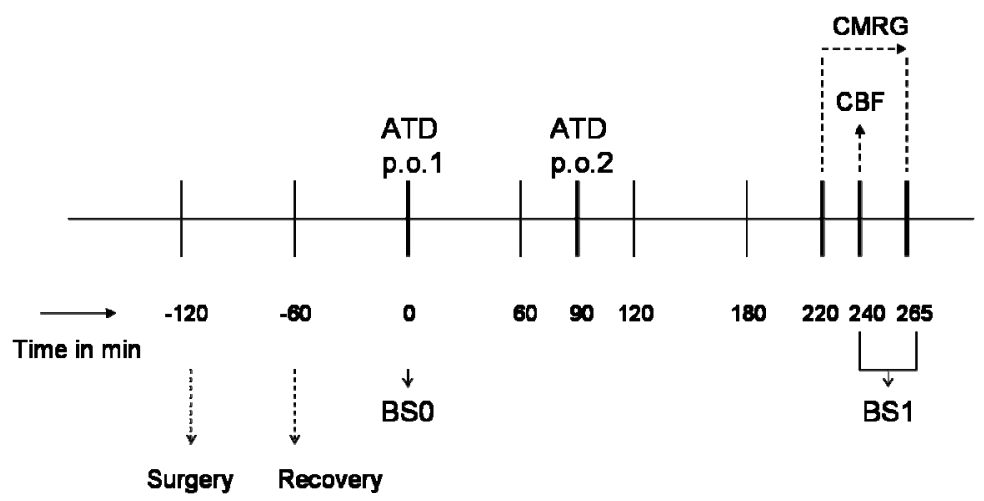

Figure 1 Schematic diagram of the different stages of the experiment. ATD: acute tryptophan depletion; p.o.: per os, oral administration by gavage; BS0: baseline blood sampling, BS1: blood sampling at the end of the experiment (t1), i.e. at either 240 (cerebral blood flow; CBF) or 265 min (cerebral metabolic rate of glucose; CMRG) after p.o.1.

\section{Measurement of local CMRG}

A total of 12 animals (TRP $+n=6$; TRP- $n=6$ ) was used for the measurement of CMRG, using the fully quantitative $\left[{ }^{14} \mathrm{C}\right]$-2-deoxyglucose autoradiographic imaging technique described previously (Sokoloff, 1981b). At $220 \mathrm{~min}$ after the first oral administration of the nutritional mixture, an intravenous injection of $\left[{ }^{14} \mathrm{C}\right]$-2-deoxyglucose $(1.3 \mathrm{MBq}$ per rat in $0.75 \mathrm{ml}$ saline; Sigma-Aldrich, UK) was administered at a constant rate over the first $30 \mathrm{~s}$ of the experiment. Over the subsequent $45 \mathrm{~min}$, a total of 14 timed arterial blood samples was collected at pre-determined intervals and centrifuged in order to separate the plasma. Aliquots $(20 \mu \mathrm{l})$ of each plasma sample were taken for the determination of $\left[{ }^{14} \mathrm{C}\right]$ and glucose by liquid scintillation analysis (Packard, Tricarb 2900TL) and semiautomated glucose oxidase enzyme assay (Beckman). At $45 \mathrm{~min}$, the animals were killed by rapid intravenous injection of sodium pentobarbitone and the brains were immediately dissected out and cut in two. One hemisphere (at random) was rapidly frozen in pre-cooled 2 -methylbutane $\left(-45^{\circ} \mathrm{C}\right)$ and mounted onto specimen holders with embedding medium (Shanon M-1; Thermo Electron Corp., Pittsburgh, PA, USA) and stored at $-80^{\circ} \mathrm{C}$ until sectioned. The other hemisphere was processed for subsequent amino acid and 5-HT determination.

\section{Measurement of local CBF}

Local cerebral blood flow was measured in a parallel group of a total of 11 rats (TRP+ $n=4$; TRP- $n=7$ ), using the quantitative $\left[{ }^{14 C}\right]$-iodoantipyrine autoradiographic imaging technique described previously (Sakurada et al., 1978). The $\left[{ }^{14} \mathrm{C}\right]$-iodoantipyrine $(1.5 \mathrm{MBq}$ per rat in $0.6 \mathrm{ml}$ saline; ARC (UK) 
Ltd, Cardiff, UK) was infused intravenously at a constantly increasing rate over $45 \mathrm{~s}$, at the end of which the animals were killed by decapitation and the brains dissected out and prepared as for the CMRG experiments. During the measurement, a maximum of 18 timed arterial blood samples was collected onto pre-weighted filter paper discs from the free flowing femoral arterial cannula. Prior to preparation for liquid scintillation analyses, the discs were reweighed, and assuming a specific gravity of 1.01, the volume of each sample was calculated.

\section{Preparation and analysis of autoradiograms}

Semi-serial sections $(20 \mu \mathrm{m})$ of the frozen cerebral hemispheres were cut in the coronal plane in a cryostat $\left(-22^{\circ} \mathrm{C}\right)$. A series of three sections were retained from every $200 \mu \mathrm{m}$, thaw mounted onto a glass coverslip and rapidly dried on a hot plate maintained at $75^{\circ} \mathrm{C}$. Autoradiograms were prepared by applying these sections to X-ray film (Kodak, SB-5), together with a series of eight precalibrated standards of known $\left[{ }^{14} \mathrm{C}\right]$ concentration $(40-1069 \mathrm{nCi} / \mathrm{g}$ tissue equivalents: Amersham International, U.K.). Films were stored in a light-tight cassette at $4^{\circ} \mathrm{C}$ for 7 days after which they were developed according to the manufacturers instructions.

Analysis of the autoradiograms was performed using a computer-based image analysis system (MCID/M5+, Imaging Research Inc., St. Catherine's, Ontario, Canada). The optical densities of autoradiographic images from the regions of interest were sampled using a measuring frame of set dimensions that were kept constant between animals. Local tissue isotope concentrations were derived from the optical density of autoradiographic brain images, relative to the $\left[{ }^{14} \mathrm{C}\right]$ standards, and $\mathrm{CMRG}$ and $\mathrm{CBF}$ were calculated from tissue $\left[{ }^{14} \mathrm{C}\right]$ concentrations and arterial tracer profiles using the appropriate operational equations (Sakurada et al., 1978; Sokoloff, 1981b). Measurements were made in a total of 43 anatomically distinct and functionally diverse brain regions identified with reference to a rat stereotaxic atlas (Paxinos and Watson, 1998) and an in-house manual (gift from professor James McCulloch) in which photographs of autoradiographic images generated from serial sections of rat brain are juxtaposed against plates from the atlas, and the exact positioning of the measurement frame for regions of interest is delineated.

\section{Blood collection and preparation for amino acid analysis}

Prior to the first gavage, a blood sample (BS) was taken for determination of baseline amino acid levels in plasma (BSO; 0 ) and a second blood sample (BS1; t1) was taken at the end of the experiment, immediately prior to sacrifice, i.e. at 240 and $265 \mathrm{~min}$ after the first oral administration by gavage for the CBF and CMRG measurements, respectively. For each blood sample, approximately 300 $\mu \mathrm{l}$ of venous blood was taken via the femoral cannulae and collected in sodium 
heparin coated tubes (Microvette ${ }^{\circledR}$ CB 300, Sarstedt, Germany). The sample tubes were kept on ice until centrifuged for $10 \mathrm{~min}(14,000 \mathrm{x} \mathrm{g})$ in order to separate the plasma. Aliquots of plasma were removed and stored at $-80{ }^{\circ} \mathrm{C}$ for subsequent amino acid determination.

Immediately prior to analysis, plasma samples were thawed to $4{ }^{\circ} \mathrm{C}$ and deproteinized in cups containing $(17 \mu \mathrm{l}$ of $5 \mathrm{mg} / 10 \mathrm{ml})$ dry 5-sulfosalicylic acid (SSA; Merck, Darmstadt, Germany). Samples were vigorously vortex-mixed and then centrifuged for $10 \mathrm{~min}(50,000 \mathrm{x}$ g). From the clear supernatant a $20 \mu \mathrm{l}$ aliquot was mixed with $1960 \mu$ distilled water to which $20 \mu$ l norvaline had been added, and stored in a temperature-maintained $\left(\begin{array}{lll}7 & { }^{\circ} \mathrm{C}\end{array}\right)$ sample compartment until high-performance liquid chromatography (HPLC) analysis.

\section{Brain tissue collection and preparation for amino acid and 5-HT analysis}

From the brain hemispheres collected at the end of the CBF and CMRG measurements, prefrontal cortex and hippocampus were dissected out onto dry ice and immediately stored at $-80^{\circ} \mathrm{C}$. For central amino acid determination, the frozen brain structures were transferred into pre-weighed $2 \mathrm{ml}$ screw-lid cups and exact tissue weight was determined with an analytic balance. Approximately $0.3 \mathrm{~g}$ of glass beads ( $1 \mathrm{~mm}$ diameter) were added to the cup together with icecold $150 \mathrm{mM} \mathrm{H} 3$ PO4 and $500 \mu \mathrm{M}$ diethylene triamine pentaacetic acid. Samples were aerated with Argon and homogenized in a mini-bead beater set at high speed for $30 \mathrm{~s}$. After a subsequent $3 \mathrm{~min}$ spin $\left(4000 \mathrm{xg}\right.$ at $\left.4^{\circ} \mathrm{C}\right)$, the homogenate was transferred back into an eppendorf vial and centrifuged for a further $20 \mathrm{~min}\left(35,000 \mathrm{x} \mathrm{g}\right.$ at $\left.4^{\circ} \mathrm{C}\right)$. The resulting supernatant was filtered through Millipore Ultrafree-MC filter cups for $1-2 \mathrm{hr}\left(9000 \mathrm{xg}\right.$ at $\left.4^{\circ} \mathrm{C}\right)$. Finally, $15 \mu$ of $2 \mathrm{mM}$ ascorbic acid was added to further protect against oxidative degradation processes, after which the samples were stored at $-80^{\circ} \mathrm{C}$ until HPLC analyses.

Plasma and brain amino acid concentrations were quantified using a fully automated HPLC system after precolumn derivatization with o-phthaldialdehyde (Domek-Lopacinska and Strosznajder; van Eijk et al., 1993). Fluorescence detection was used to quantify OPA-AA derivates of the amino acids. Central concentrations of 5-HT and the 5-HT metabolite (5-hydroxyindoleacetic acid; 5-HIAA) in hippocampus and prefrontal cortex were quantified at a later stage in the remaining supernatant by automated HPLC with dual electrochemical detection. The concentrations of total plasma and central amino acid concentrations were expressed as $\mu \mathrm{mol} / \mathrm{l}$, brain concentrations as $\mathrm{ng} / \mathrm{mg}$. 


\section{Statistical analysis}

All data are represented as mean \pm standard error of the mean (S.E.M.) and unless otherwise stated, data sets were evaluated statistically using unpaired Student's $t$-test. In all cases, acceptable levels of significance were set at $P<0.05$.

Differences between TRP + and TRP- treatment groups and time effects ( $t 0$ versus $t 1$, of which the latter is the mean of 240 and 265 min measurements) within treatment groups were statistically evaluated for all absolute plasma LNAA concentrations as well as arginine (ARG) and citrulline (CIT). The plasma ratio TRP $/ \Sigma$ LNAA was calculated and expressed as percentage change from its corresponding baseline value. Central amino acid concentrations, 5-HT and 5-HIAA levels including 5-HT turnover rate were analyzed for hippocampus and prefrontal cortex separately. Extreme values in the plasma and brain biochemical data were calculated by means of Dixon's Extremity test (Dixon, 1959) and excluded from statistical analysis. Analyses were performed using the Statistical Package for Social Science (SPSS) for Windows (Rel.12.0.1.2003, Chicago: SPSS Inc.).

Statistical analyses of local $\mathrm{CBF}$ and $\mathrm{CMRG}$ data were performed by Student's $t$-test with acceptable levels of significance set at $P<0.05$. To provide an initial assessment of differences between treatment groups in the relationship of local CBF to local CMRG in each of 43 regions of interest (ROI) included in this study, the ratios of mean local CBF to mean local CMRG derived from each structure were analysed by Mann-Whitney $U$ test using the curve fitting program GraphPad Prism, version 4.02 for Windows (GraphPad Software, San Diego California USA). More rigorous statistical analysis of the relationship was performed by repeated measures analysis of variance on the log transform of the data sets (McCulloch et al., 1982) using the BMDP/PC90 computerized statistical package (program 2V) (Jennrich et al., 1990). In this analysis, "type of variable" was designated the grouping factor, and "brain region" was designated the repeated measures trial factor. While the experimental design was insufficient to allow a full multivariate profile analysis, inter-regional differences in the magnitude of the ratio of local CBF to local CMRG could be examined using an Univariate approach (Ford et al., 1991; Geiser and Greenhouse, 1958).

\section{Results}

\section{Physiological parameters}

There were no significant differences in physiological parameters, either between treatment groups, within treatment groups (before and after treatment), or between animals with similar treatments used for either CBF or CMRG experiments. In particular, there were no significant differences in 
plasma glucose levels between the groups of rats before treatment $(8.7 \pm 0.7$ mmol.L-1 and $8.6 \pm 0.8 \mathrm{mmol}^{-1} \mathrm{~L}^{-1}$ in TRP- and TRP+ groups, respectively) or at the time of the final experiment $\left(8.9 \pm 1.4 \mathrm{mmol}^{-1}\right.$ and $8.9 \pm 1.8 \mathrm{mmol}^{-1} \mathrm{~L}^{-1}$ in TRP- and TRP+ groups, respectively), and these remained steady throughout the measurement periods.

\section{Plasma amino acid concentrations}

Plasma levels of the LNAAs, as well as ARG and CIT were measured both prior to the first oral administration (baseline; $t 0$ ) and at the end of the experiment (t1). Since no difference was found between either the plasma or brain biochemical results at $\mathrm{t} 1=240 \mathrm{~min}(\mathrm{CBF})$ and at $\mathrm{t} 1=265 \mathrm{~min}(\mathrm{CMRG})$ after the first oral administration, data were pooled to increase statistical power.

At baseline, no differences were observed in the LNAA concentrations between the subsequent treatment groups (Table 2). At the end of the experimental period (t1), the concentration of TRP was found to be significantly reduced $(-33 \%)$ from baseline levels in the TRP-group $[t(23)=$ 3.723; $P<0.01]$ and was also significantly lower $(-38 \%)$ compared to the TRP+ group $[t(21)=5.317 ; P<0.01]$.

Table 2 Absolute plasma amino acid (AA) concentrations in the two treatment groups (mean \pm S.E.M. in $\mu \mathrm{mol} / \mathrm{l}$ ) at baseline ( $\mathrm{t} 0)$ and at the end of the experiments ( $\mathrm{t} 1$ ) (pooled for $\mathrm{CBF}$ and CMRG experiments; TRP+; $n=10$, TRP-; $n=9)$.

\begin{tabular}{lcccc}
\hline Amino acid & Baseline $(\mathrm{t} 0)$ & \multicolumn{2}{c}{ End of experiment $(\mathrm{t} 1)$} \\
\cline { 2 - 4 } & TRP+ & TRP- & TRP+ & TRP- \\
TRP & $57 \pm 3$ & $54 \pm 4$ & $58 \pm 3$ & $36 \pm 2^{*, a}$ \\
TYR & $74 \pm 5$ & $71 \pm 5$ & $74 \pm 6$ & $69 \pm 3$ \\
PHE & $65 \pm 2$ & $64 \pm 2$ & $92 \pm 2^{\mathrm{a}}$ & $89 \pm 2^{\mathrm{a}}$ \\
VAL & $186 \pm 9$ & $187 \pm 6$ & $229 \pm 10^{\mathrm{a}}$ & $237 \pm 8^{\mathrm{a}}$ \\
ILE & $100 \pm 5$ & $104 \pm 4$ & $88 \pm 2^{\mathrm{a}}$ & $88 \pm 3^{\mathrm{a}}$ \\
LEU & $133 \pm 6$ & $140 \pm 6$ & $154 \pm 4^{\mathrm{a}}$ & $156 \pm 6$ \\
ARG & $157 \pm 10$ & $177 \pm 8$ & $163 \pm 10$ & $155 \pm 6$ \\
CIT & $70 \pm 2$ & $68 \pm 2$ & $84 \pm 3^{\mathrm{a}}$ & $79 \pm 6^{\mathrm{a}}$ \\
\hline
\end{tabular}

$\left.{ }^{*}\right)$ indicates significant difference in AA concentration between the TRP+ and TRP- group. ${ }^{a}$ ) indicates significant change over time $(*$, a) $P<0.05$. TRP: tryptophan, TYR: tyrosine, PHE: phenylalanine, VAL: valine, ILE: isoleucine, LEU: leucine, ARG: arginine, CIT: citrulline. 
Some of the other AAs included in the analyses (PHE, VAL and CIT) were found to be significantly increased at $\mathrm{t} 1$ when compared to $\mathrm{t} 0 \mathrm{in}$ both treatment groups, while a significant increase in LEU was found only in the TRP+ treatment group. Significant decreases in ILE between t 0 and $\mathrm{t} 1$ were found in both TRP+ and TRP- treatment groups (Table 2), but the reductions $(-12 \%$ and $-15 \%$, respectively) were less marked than the treatment effects on TRP levels.

Analysis of data in the form of ratios between TRP in plasma to the sum of all LNAAs with which TRP competes for transport across the blood-brain barrier (TRP $/ \Sigma$ LNAA) revealed a significant decrease (-40\%; Fig. 2) from baseline in the TRP-group $[t(20)=4.534 ; P<0.01]$. A decrease in the ratio TRP $/ \Sigma L N A A$ from baseline levels was also found following TRP+ treatment, but this was considerably smaller in magnitude $(-8 \%)$ and was not significant $[t(22)=2.033$; n.s.].

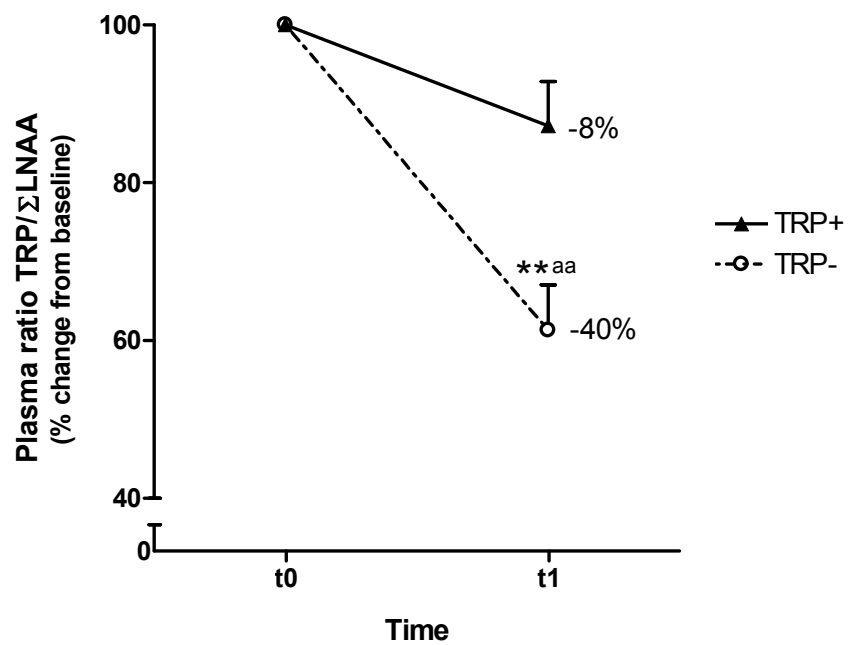

Figure 2 Percentage change of the ratio TRP $/ \Sigma$ LNAA in plasma compared to baseline levels (mean + S.E.M.), 240-265 min after the first oral administration with the protein-carbohydrate nutritional mixture with $(\mathrm{TRP}+; \mathrm{n}=10)$ or without TRP $($ TRP-; $n=9) .\left(^{*}\right)$ significantly different from TRP+ group, (a) significantly different over time (**, aa) $P<0.01$

\section{Brain amino acids, 5-HT and 5-HIAA concentrations}

Despite there being significant reductions in plasma TRP following ATD, no significant differences in TRP levels were found in either hippocampus $[t(8)=$ 0.156 ; n.s.] or prefrontal cortex $[t(11)=0.935$; n.s.] in the TRP- treatment group when compared to TRP+ controls (Table 3). In general, none of the 
AAs appeared to be centrally affected by ATD, except for CIT where in the TRP-group, a significant decrease $(-17 \%)$ was found in hippocampal CIT levels when compared to the TRP+ group $[t(11)=2.198 ; P=0.05]$. In line with the unaltered brain TRP levels, no differences were found in 5-HT concentrations in hippocampus $[t(9)=0.505$; n.s. or prefrontal cortex $[t(8)=0.245$; n.s. $]$ after ATD compared to the TRP+ control group and also no ATD-induced changes were detected in 5-HIAA levels in hippocampus $[t(11)=0.354$; n.s. $]$ or prefrontal cortex $[t(11)=0.377$; n.s. $]$ (Table 3$)$.

Table 3 Absolute central amino acid (AA) concentrations and central 5-HT, 5-HIAA and 5-HT turnover (mean \pm S.E.M. in $\mathrm{ng} / \mathrm{mg}$ ) 240-265 min (t1; pooled for CBF and CMRG experiments) after the first oral administration with the protein-carbohydrate nutritional mixture with (TRP+; $n=10)$ or without TRP (TRP-; $n=9)$ in rat hippocampus and prefrontal cortex.

\begin{tabular}{lcccc}
\hline & \multicolumn{3}{c}{ Hippocampus } & \multicolumn{3}{c}{ Prefrontal cortex } \\
\cline { 2 - 5 } & TRP+ & TRP- & TRP+ & TRP- \\
\hline TRP & $22 \pm 0.5$ & $22 \pm 0.7$ & $22 \pm 1.1$ & $20 \pm 1.4$ \\
TYR & $13 \pm 0.6$ & $13 \pm 1.1$ & $13 \pm 1$ & $13 \pm 0.8$ \\
PHE & $19 \pm 0.6$ & $19 \pm 0.4$ & $20 \pm 0.9$ & $19 \pm 0.7$ \\
VAL & $17 \pm 0.6$ & $15 \pm 1.2$ & $17 \pm 1.8$ & $16 \pm 0.6$ \\
ILE & $11 \pm 0.8$ & $11 \pm 0.5$ & $11 \pm 0.7$ & $11 \pm 0.7$ \\
LEU & $14 \pm 0.8$ & $12 \pm 0.2$ & $15 \pm 0.7$ & $14 \pm 0.7$ \\
ARG & $35 \pm 3.7$ & $29 \pm 3.4$ & $21 \pm 2$ & $28 \pm 4.8$ \\
CIT & $12 \pm 0.8$ & $10 \pm 0.4 *$ & $9 \pm 0.6$ & $7 \pm 1.3$ \\
5-HT & $0.30 \pm 0.04$ & $0.28 \pm 0.01$ & $0.23 \pm 0.03$ & $0.22 \pm 0.02$ \\
5-HIAA & $0.27 \pm 0.04$ & $0.24 \pm 0.05$ & $0.26 \pm 0.03$ & $0.24 \pm 0.05$ \\
5-HT turnover & $0.95 \pm 0.09$ & $0.90 \pm 0.23$ & $1.27 \pm 0.09$ & $1.04 \pm 0.16$ \\
\hline
\end{tabular}

$\left.{ }^{*}\right)$ indicates significant difference in AA concentration between the TRP+ and TRP-group $(P<0.05)$. TRP: tryptophan, TYR: tyrosine, PHE: phenylalanine, VAL: valine, ILE: isoleucine, LEU: leucine, ARG: arginine, CIT: citrulline, 5-HT: 5-hydroxytryptamine, 5-HIAA: 5hydroxyindoleacetic acid and 5-HT turnover: 5-HIAA/5-HT

\section{Local cerebral metabolic rate of glucose}

Although ATD produced a tendency towards decreased CMRG in the majority of the 43 brain ROIs analysed, none of them were statistically significant (Table 4).

\section{Local cerebral blood flow}

ATD induced generalised decrease in CBF when compared to the TRP+ treatment group, and in 11 of the 43 brain areas analysed these decreases were 
statistically significant (Table 4). Decreases in CBF were found throughout the hippocampal formation (molecular layer, $-26 \%$; dentate PO, $-22 \%$; dorsal CA1, $-25 \%$; CA2, $-26 \%$; CA3, $-26 \%$ ), but elsewhere in the brain CBF was also decreased in the globus pallidus, $(-27 \%)$, lateral amygdala $(-17 \%)$, ventrolateral thalamic nucleus $(-22 \%)$, ventral tegmental area $(-26 \%)$, and entorhinal cortex $(-21 \%)$. The most profound decrease in CBF was measured in temperoparietal cortex $(-40 \%)$ (Table 4$)$.

Table 4 Local cerebral metabolic rate of glucose (CMRG, $\mu \mathrm{mol} / 100 \mathrm{~g} / \mathrm{min}$ ) and local cerebral blood flow (CBF, $\mathrm{ml} / 100 \mathrm{~g} / \mathrm{min}$ ) 265 and $240 \mathrm{~min}$, respectively, after the first oral administration with the protein-carbohydrate nutritional mixture with $(T R P+; n=6$ and $n=4$ for $C M R G$ and $\mathrm{CBF}$, respectively) or without TRP (TRP-; $n=6$ and $n=7$ for CMRG and CBF, respectively). Data are presented as mean \pm S.E.M. The flow-metabolism coupling ratio (CBF/CMRG) was calculated from mean values for each brain area.

\begin{tabular}{|c|c|c|c|c|c|c|}
\hline \multirow[t]{2}{*}{ Brain regions } & \multicolumn{2}{|l|}{ CMRG } & \multicolumn{2}{|l|}{$\mathrm{CBF}$} & \multicolumn{2}{|c|}{$\begin{array}{l}\text { Ratio } \\
\text { CBF/CRMG }\end{array}$} \\
\hline & $\begin{array}{l}\text { TRP+ } \\
(n=6)\end{array}$ & $\begin{array}{l}\text { TRP- } \\
(n=6)\end{array}$ & $\begin{array}{l}\text { TRP+ } \\
(n=4)\end{array}$ & $\begin{array}{l}\text { TRP- } \\
(n=7)\end{array}$ & TRP+ & TRP- \\
\hline Frontal cortex & $106 \pm 12$ & $98 \pm 5$ & $109 \pm 16$ & $89 \pm 8$ & 1.03 & 0.91 \\
\hline Orbital frontal cortex & $142 \pm 16$ & $134 \pm 8$ & $168 \pm 18$ & $143 \pm 13$ & 1.18 & 1.06 \\
\hline Dorsal medial prefrontal cortex & $75 \pm 8$ & $76 \pm 3$ & $95 \pm 10$ & $78 \pm 7$ & 1.26 & 1.03 \\
\hline Ventral medial prefrontal cortex & $109 \pm 12$ & $109 \pm 7$ & $125 \pm 16$ & $99 \pm 14$ & 1.15 & 0.91 \\
\hline Nucleus accumbens & $88 \pm 9$ & $84 \pm 3$ & $121 \pm 14$ & $104 \pm 9$ & 1.38 & 1.24 \\
\hline Corpus callosum & $22 \pm 3$ & $19 \pm 7$ & $33 \pm 5$ & $28 \pm 1$ & 1.52 & 1.47 \\
\hline Somatosensory cortex & $119 \pm 10$ & $113 \pm 6$ & $151 \pm 29$ & $114 \pm 7$ & 1.27 & 1.01 \\
\hline Anterior cingulate cortex & $99 \pm 10$ & $98 \pm 5$ & $117 \pm 17$ & $95 \pm 7$ & 1.18 & 0.97 \\
\hline Septal nucleus & $61 \pm 7$ & $55 \pm 4$ & $74 \pm 11$ & $66 \pm 4$ & 1.21 & 1.20 \\
\hline Medial striatum & $94 \pm 9$ & $92 \pm 6$ & $99 \pm 13$ & $87 \pm 4$ & 1.06 & 0.95 \\
\hline Lateral striatum & $106 \pm 11$ & $106 \pm 5$ & $111 \pm 14$ & $95 \pm 5$ & 1.05 & 0.90 \\
\hline Bed nucleus of striae terminalis & $46 \pm 5$ & $45 \pm 4$ & $56 \pm 5$ & $48 \pm 3$ & 1.22 & 1.06 \\
\hline Globus pallidus & $54 \pm 7$ & $50 \pm 3$ & $65 \pm 7$ & $47 \pm 3^{*} \downarrow$ & 1.21 & 0.95 \\
\hline Antero ventral thalamic nucleus & $105 \pm 11$ & $97 \pm 12$ & $133 \pm 9$ & $109 \pm 8$ & 1.27 & 1.12 \\
\hline Antero hypothalamic nucleus & $53 \pm 7$ & $58 \pm 9$ & $74 \pm 9$ & $62 \pm 5$ & 1.38 & 1.08 \\
\hline Tempero parietal cortex & $114 \pm 11$ & $109 \pm 5$ & $202 \pm 32$ & $121 \pm 8^{*} \downarrow$ & 1.77 & 1.11 \\
\hline Ventral lateral hypothalamic nucleus & $62 \pm 7$ & $52 \pm 3$ & $82 \pm 5$ & $70 \pm 5$ & 1.34 & 1.35 \\
\hline Medial thalamic nucleus & $100 \pm 8$ & $95 \pm 3$ & $127 \pm 15$ & $109 \pm 8$ & 1.27 & 1.14 \\
\hline Ventral lateral thalamic nucleus & $88 \pm 9$ & $83 \pm 4$ & $128 \pm 11$ & $100 \pm 6^{*} \downarrow$ & 1.45 & 1.20 \\
\hline Medial amygdala & $84 \pm 10$ & $79 \pm 5$ & $101 \pm 7$ & $80 \pm 6$ & 1.20 & 1.01 \\
\hline Lateral amygdala & $52 \pm 5$ & $49 \pm 2$ & $79 \pm 4$ & $66 \pm 3^{*} \downarrow$ & 1.52 & 1.35 \\
\hline Posterior cingulate cortex & $105 \pm 10$ & $94 \pm 5$ & $126 \pm 15$ & $100 \pm 8$ & 1.20 & 1.07 \\
\hline Lateral habenula & $116 \pm 15$ & $108 \pm 6$ & $155 \pm 21$ & $121 \pm 10$ & 1.33 & 1.12 \\
\hline
\end{tabular}




\begin{tabular}{|c|c|c|c|c|c|c|}
\hline \multirow[t]{2}{*}{ Brain regions } & \multicolumn{2}{|l|}{ CMRG } & \multicolumn{2}{|l|}{$\mathrm{CBF}$} & \multicolumn{2}{|c|}{$\begin{array}{l}\text { Ratio } \\
\text { CBF/CRMG }\end{array}$} \\
\hline & $\begin{array}{l}\text { TRP+ } \\
(n=6)\end{array}$ & $\begin{array}{l}\text { TRP- } \\
(n=6)\end{array}$ & $\begin{array}{l}\text { TRP+ } \\
(n=4)\end{array}$ & $\begin{array}{l}\text { TRP- } \\
(n=7)\end{array}$ & TRP+ & TRP- \\
\hline Subthalamic nucleus & $84 \pm 7$ & $87 \pm 3$ & $149 \pm 19$ & $117 \pm 7$ & 1.78 & 1.35 \\
\hline Mamillary body & $110 \pm 12$ & $96 \pm 9$ & $141 \pm 17$ & $129 \pm 19$ & 1.29 & 1.34 \\
\hline Piriform cortex & $67 \pm 8$ & $60 \pm 3$ & $89 \pm 8$ & $73 \pm 5$ & 1.33 & 1.22 \\
\hline Entorhinal cortex & $69 \pm 9$ & $62 \pm 2$ & $81 \pm 7$ & $64 \pm 4^{*} \downarrow$ & 1.17 & 1.02 \\
\hline Molecular layer & $90 \pm 12$ & $84 \pm 5$ & $114 \pm 9$ & $84 \pm 6^{*} \downarrow$ & 1.27 & 1.01 \\
\hline Dorsal subiculum & $87 \pm 9$ & $86 \pm 5$ & $95 \pm 7$ & $82 \pm 6$ & 1.10 & 0.95 \\
\hline Dentate PO & $56 \pm 7$ & $55 \pm 4$ & $81 \pm 7$ & $63 \pm 3 * \downarrow$ & 1.44 & 1.15 \\
\hline Dorsal CA1 & $67 \pm 9$ & $61 \pm 5$ & $83 \pm 8$ & $62 \pm 4 * \downarrow$ & 1.24 & 1.02 \\
\hline CA2 & $65 \pm 10$ & $66 \pm 5$ & $105 \pm 8$ & $77 \pm 6^{*} \downarrow$ & 1.60 & 1.17 \\
\hline Ventral CA1 & $75 \pm 10$ & $64 \pm 4$ & $112 \pm 4$ & $93 \pm 9$ & 1.50 & 1.47 \\
\hline Ventral subiculum & $69 \pm 7$ & $64 \pm 3$ & $106 \pm 11$ & $92 \pm 7$ & 1.53 & 1.43 \\
\hline CA3 & $71 \pm 9$ & $70 \pm 3$ & $103 \pm 7$ & $76 \pm 3^{*} \downarrow$ & 1.44 & 1.09 \\
\hline Ventral tegmental area & $63 \pm 9$ & $61 \pm 6$ & $78 \pm 7$ & $58 \pm 3^{*} \downarrow$ & 1.24 & 0.95 \\
\hline Substantia nigra reticulata & $59 \pm 6$ & $54 \pm 3$ & $80 \pm 6$ & $70 \pm 4$ & 1.35 & 1.29 \\
\hline Substantia nigra compacta & $76 \pm 10$ & $72 \pm 4$ & $93 \pm 4$ & $80 \pm 4$ & 1.22 & 1.11 \\
\hline Dorsal raphe & $74 \pm 9$ & $80 \pm 6$ & $106 \pm 15$ & $95 \pm 12$ & 1.44 & 1.19 \\
\hline Median raphe & $83 \pm 8$ & $75 \pm 9$ & $119 \pm 28$ & $109 \pm 11$ & 1.44 & 1.45 \\
\hline Paramedian raphe & $71 \pm 11$ & $64 \pm 7$ & $96 \pm 23$ & $106 \pm 11$ & 1.36 & 1.65 \\
\hline Inferior colliculus & $203 \pm 26$ & $178 \pm 5$ & $282 \pm 62$ & $229 \pm 12$ & 1.39 & 1.28 \\
\hline Locus coeruleus & $77 \pm 9$ & $64 \pm 4$ & $112 \pm 29$ & $83 \pm 4$ & 1.44 & 1.30 \\
\hline Mean ratio & & & & & 1.33 & $1.15^{*}$ \\
\hline
\end{tabular}

$\left(^{*}\right)$ indicates significant decreases in $\mathrm{CBF}$ after ATD compared to TRP+ control treatment * $P<0.05$. A significant decrease in the overall ratio was found after ATD compared to the TRP+ control group.

\section{Relationship between $\mathrm{CBF}$ and $\mathrm{CMRG}$}

Decreases in CBF in response to ATD in the absence of any changes of similar magnitude in CMRG (Table 4), had marked effects upon the flow-metabolism coupling relationship normally found in the brain. In TRP+ control rats, there was clearly an association between $\mathrm{CBF}$ and mean CMRG in the brain as a whole $(r=0.93)$ and the overall ratio between blood flow and glucose use, as illustrated by the slope of the best-fitting straight line, was 1.27 (Fig. 3). In those animals treated with ATD, the relationship between CBF and CMRG remained largely intact $(r=0.91)$ (Fig. 3), although the slope of the line defining the overall relationship $(\mathrm{m}=1.07)$ was lower than in the control group (Fig. 3) and indicated a degree of tissue oligaemia. Examining the brain areas 
individually, the flow-metabolism ratio was found to decrease in all but 4 of the 43 brain areas in ATD-treated rats (Table 4), and over the brain as a whole analyses of the ratios (Mann-Whitney U-test) indicated a significant decrease in the ATD group (mean ratio $=1.15$ ) when compared to controls (mean ratio $=$ 1.33).

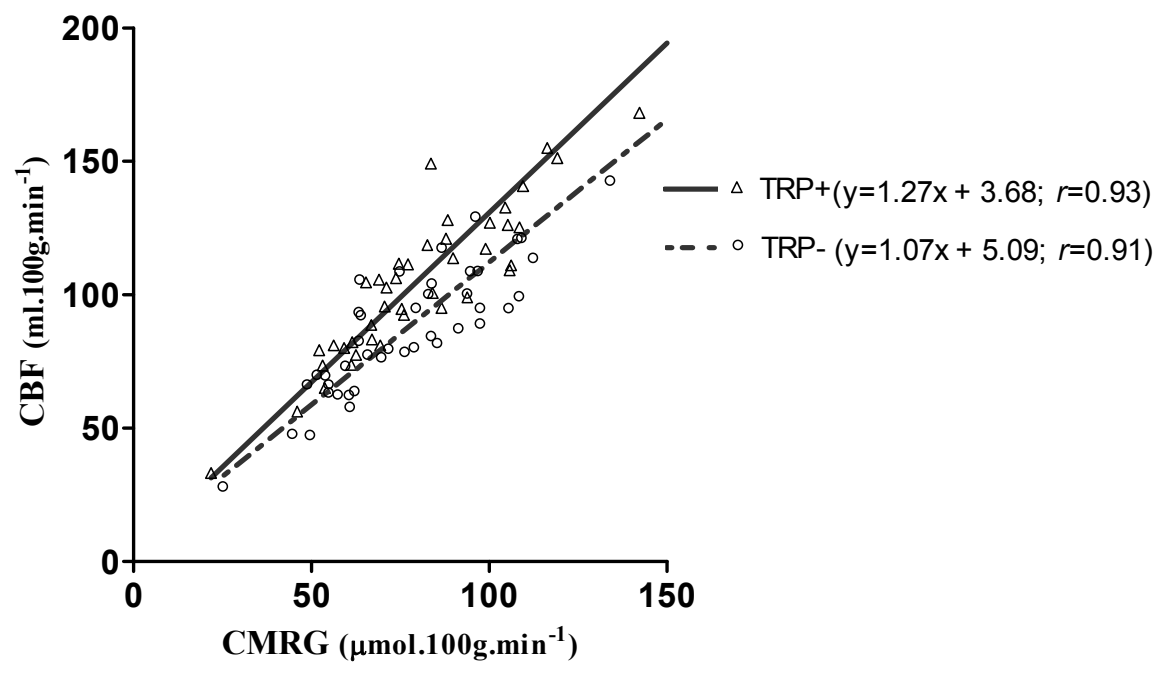

Figure 3 The relationship between local cerebral blood flow (CBF) and local cerebral metabolic rate of glucose (CMRG) using data generated from all 43 brain regions of interest after ATD and TRP+ treatment. Data points represent mean CBF and CMRG for each region together with the best fitting straight line defined by $\mathrm{y}=\mathrm{mx}+\mathrm{c}$, where $\mathrm{x}$ and $\mathrm{y}$ are the variables $(\mathrm{CMRG}$ and $\mathrm{CBF}$, respectively), $\mathrm{m}=$ the slope of the line and $\mathrm{c}=$ the intercept.

\section{Discussion}

The conversion of the essential amino acid TRP to 5-hydroxytrpytophan by isoforms of the enzyme tryptophan hydroxylase is the first step in the biosynthesis of 5-HT. In the brain, this enzyme is only 50\% saturated, and therefore the rate at which 5-HT is synthesised is limited only by substrate (TRP) availability. The ATD challenge method is thus a relatively easy procedure with which to rapidly and reversibly decrease circulating TRP concentrations, and thereby decrease substrate availability for synthesis into 5-HT both peripherally and in the brain. Previous rat studies of similar experimental design to that used here, including the same nutritional mixture (Cahir et al., 2008; Jans et al., 2007a; Lieben et al., 2004a), found profound decreases in the ratio TRP/ $/$ LNAA in plasma following ATD in Wistar rats 4 $\mathrm{h}$ after the first administration, ranging from $-60 \%$ to $-78 \%$. However, less 
robust central effects were reported ranging from $-17 \%$ (non-significant) to $-66 \%$ in hippocampus and $-20 \%$ (non-significant) to $-70 \%$ in frontal cortex. Moreover, even where significant effects were found in brain tissue, the response was often variable, with coefficients of variation in some instances approaching $50 \%$.

In the present study, we induced an acute depletion of plasma TRP levels in exactly the same way as reported previously, by administering $10 \mathrm{ml} / \mathrm{kg}$, twice with a 90 min interval, of a TRP-free protein-carbohydrate nutritional mixture. Around $4 \mathrm{~h}$ after the first oral administration, this treatment resulted in a significant $40 \%$ decrease in the plasma TRP/ $\Sigma$ LNAA ratio from baseline levels, which compares favourably to the $8 \%$ decrease in the TRP+ treated control group. It remains unclear why only a moderate reduction in peripheral TRP was found in the present study. Most of the significant peripheral changes of other amino acids over time in both treatment groups, as well as the slight reduction in the TRP/ $/ \mathrm{LNAA}$ ratio after TRP+ treatment, are likely to be attributed to the boost of specific amino acids following administration of the nutritional mixture. However, in the present study the ATD treatment did not have any significant effect upon TRP and 5-HT levels in either hippocampal or prefrontal cortical tissue. Also the 5-HIAA concentrations remained unaffected after ATD. This is perhaps not surprising given that more pronounced decreases in plasma TRP $/ \Sigma$ LNAA ratios reported previously failed to induce consistent inter-study effects in these two brain areas. Further analysis of brain tissue revealed that ATD had no significant effect upon other central amino acid concentration, with the exception of CIT where a decrease in hippocampal CIT concentration was found only in the TRP-group. A similar decrease in the central concentrations of CIT following ATD have also been observed previously by others Lieben et al. (2004a), and given that ARG is catalysed into CIT and nitric oxide (NO) (Dawson and Dawson, 1996), it has been proposed that decreases in CIT might parallel decreases in NO synthesis (NOS) and that such a mechanism might underlie the effects that ATD has upon memory processing (Prast and Philippu, 2001), and could also influence cerebral blood vessels (Kelly et al., 1994).

Under normal physiological conditions, the rate of glucose metabolism in the brain provides an index of changes in regional neuronal activity and changes in glucose metabolism are found to be closely coupled to changes in CBF (Kuschinsky, 1991; Sokoloff, 1981a). On this basis, abnormalities in either of these closely linked neurophysiological parameters in depressive subjects are thought to reflect changes in 5-HT neurotransmission in brain areas that can be functionally linked to the complexity of depressive symptomatology displayed (Drevets et al., 1998a, 1998b; Drevets et al., 1999; Drevets, 2000). Certainly in animal studies, acute pharmacological manipulations of central 5-HT systems have previously been found to induce alterations in glucose utilization (Grasby et al., 1992; Kelly et al., 1988), but changes in CBF are not always found to be directly related, either quantitatively or qualitatively, to changes in underlying 
metabolic demand (Quate et al., 2004). This uncoupling has been attributed to the fact that 5-HT possesses potent vasoactive properties (Edvinsson and MacKenzie, 1976) and 5-HT fibers have been identified innervating cerebral arteries, arterioles and veins (Steinbusch, 1981). Thus, there is the potential for 5-HT to play an important role in the regulation and modulation of haemodynamic processes, independent of underlying metabolism, through vasoconstriction of cerebral blood vessels and consequent decreases in blood flow (Bonvento et al., 1991; Cohen et al., 1996; Kelly et al., 1988; McBean et al., 1991).

Although it was anticipated that depletion of 5-HT by ATD might alter neuronal function and metabolic activity in the brain, the absence of any changes in CMRG in the TRP-group is certainly in keeping with the lack of treatment-induced change in central 5-HT in this study. In contrast, the ATD treatment produced significant effects upon $\mathrm{CBF}$, notably in hippocampus and cortex which were the areas used for TRP analysis. In these areas, significant decreases in $\mathrm{CBF}$ were measured, indicating either an increase in constrictor tone or loss of dilator tone, rather than the loss of serotonergic constrictor that might have been expected following ATD. Interestingly, decreases in CBF after ATD have also been found in the dorsal anterior cingulate cortex of healthy human subjects, which has been attributed to differential effects of reduced 5-HT upon ventral and dorsal neural systems (Talbot and Cooper, 2006). A similar mechanism could be responsible for the effects on CBF found in this study, and if ATD was more effective at reducing the availability of 5-HT at serotonergic synapses in the raphe than at projection terminals, the resultant disinhibition of raphe neurons would increase cerebrovascular constrictor tone and produce the reductions in $\mathrm{CBF}$ that were in fact measured. Given the small mass of raphe tissue, it would be difficult to verify if such mechanisms were in action, although perhaps electrophysiological analysis of raphe function following ATD might provide some further insides. Alternatively, the decreases in CBF could be explained by a loss of dilator tone, as found for example when either endothelial or neuronal NOS is inhibited (Kelly et al., 1994; Kelly et al., 1995). The involvement of NO might be suggested by the changes in brain levels of CIT as described earlier, but whatever the underlying mechanism, the decreases in CBF in this study following ATD treatment in the absence of a qualitatively similar change in CMRG, represents a state of cerebral oligaemia with significant downwards resetting of mean flow-metabolism ratios (from 1.33 to 1.15$)$.

While considerable evidence exists for ATD-induced decreased central 5-HT availability in both human subjects (e.g. Carpenter et al., 1998; Williams et al., 1999) and animals (e.g. Biggio et al., 1974; Lieben et al., 2004a; Moja et al., 1989), the extent to which a decrease in 5-HT synthesis results in disturbed 5-HT neuronal activity remains unclear. An actual reduction of extracellular 5-HT after ATD has only been reported in combination with 5-HT reuptake inhibitors (Bel and Artigas, 1996; Fadda et al., 2000b; Stancampiano et al., 
1997). It is suggested that the use of 5-HT reuptake blockade is necessary to raise 5-HT to optimal levels for detection. It might also suggest, however, that in the absence of de novo synthesis, 5-HT function can be largely maintained from transmitter being recycled into the presynaptic cell from the synapse. Furthermore, it appears that basal 5-HT release in the prefrontal cortex of rats is not affected by ATD (van der Plasse et al., 2007). In line with this, the results of the present study indicate that the underlying mechanism of the ATD method possibly goes beyond a straightforward alteration in 5-HT activity.

In the present study, the acute decrease of peripheral TRP levels resulted in a downwards resetting of the flow-metabolism coupling relationship in the brain, as indicated by the decrease in the mean ratio of metabolism to blood flow over all brain areas. This parallels preliminary findings of an uncoupling of flow from metabolism in unipolar depressed patients compared to bipolar patients and healthy controls (Dunn et al., 2005). However, controversy exists about the exact location and direction of the neurophysiological abnormalities in depressive subjects (Drevets, 2000; Soares and Mann, 1997), although depressive illness is generally characterized by decreases in cerebral blood flow and glucose metabolism in limbic and prefrontal cortex structures. As outlined previously though, it seems unlikely that a decrease in 5-HT alone accounts for the specific decreases in haemodynamic regulation, which also supports the notion that depression in general cannot be simplistically described as a plain serotonergic dysfunctional disorder.

In summary, despite the extensive use of the ATD method in depression research, the exact mechanism through which ATD exerts its neurophysiological effect is still poorly understood. Consequently, to what extent the ATD induced functional and behavioural alterations can be directly attributed to changes in 5-HT neuronal activity remains uncertain. In the present study, the ATD-induced decrease in peripheral TRP levels resulted in a resetting of the normal relationship between local CBF and CMRG, indicating a state of relative cerebral oligaemia, without affecting central TRP levels. Therefore, a more additive role for a dysfunctional 5-HT system as an underlying mechanism of the ATD method might be implied. Additionally, cerebrovascular dysfunction might be a potential contributory factor underlying the onset of depressive symptomatology, including cognitive deficiencies. More generally, whereas the precise direction of flow- metabolism coupling alterations in depression remains uncertain, the results of the present study have important implications for imaging studies that use CBF alone as a measure of neuronal function.

\section{Acknowledgements}

This work was funded by a EUROGENDIS award to E.L.v.D. We would like to thank Dr. Douglas E. McBean (Queen Margaret University, Edinburgh) for 
his help in the statistical analysis of the relationship between cerebral blood flow and metabolism.

\section{References}

Bel N, Artigas F (1996). Reduction of serotonergic function in rat brain by tryptophan depletion: effects in control and fluvoxamine-treated rats. J Neurochem 67(2): 669-676.

Bell CJ, Hood SD, Nutt DJ (2005). Acute tryptophan depletion. Part II: clinical effects and implications. Aust N Z J Psychiatry 39(7): 565-574.

Biggio G, Fadda F, Fanni P, Tagliamonte A, Gessa GL (1974). Rapid depletion of serum tryptophan, brain tryptophan, serotonin and 5-hydroxyindoleacetic acid by a tryptophan-free diet. Life Sci 14(7): 1321-1329.

Blokland A, Lieben C, Deutz NE (2002). Anxiogenic and depressive-like effects, but no cognitive deficits, after repeated moderate tryptophan depletion in the rat. J Psychopharmacol 16(1): 39-49.

Bonvento G, MacKenzie ET, Edvinsson L (1991). Serotonergic innervation of the cerebral vasculature: relevance to migraine and ischaemia. Brain Res Brain Res Rev 16(3): 257-263.

Booij L, Van der Does W, Benkelfat C, Bremner JD, Cowen PJ, Fava M, Gillin C, Leyton M, Moore P, Smith KA, Van der Kloot WA (2002). Predictors of mood response to acute tryptophan depletion. A reanalysis. Neuropsychopharmacology 27(5): 852-861.

Booij L, Van der Does AJ, Riedel WJ (2003). Monoamine depletion in psychiatric and healthy populations: review. Mol Psychiatry 8(12): 951-973.

Booij L, Van der Does AJ, Haffmans PM, Riedel WJ, Fekkes D, Blom MJ (2005a). The effects of high-dose and low-dose tryptophan depletion on mood and cognitive functions of remitted depressed patients. J Psychopharmacol 19(3): 267-275.

Booij L, van der Does AJ, Haffmans PM, Spinhoven P, McNally RJ (2005b). Acute tryptophan depletion as a model of depressive relapse: behavioural specificity and ethical considerations. Br J Psychiatry 187: 148-154.

Bremner JD, Innis RB, Salomon RM, Staib LH, Ng CK, Miller HL, Bronen RA, Krystal JH, Duncan J, Rich D, Price LH, Malison R, Dey H, Soufer R, Charney DS (1997). Positron emission tomography measurement of cerebral metabolic correlates of tryptophan depletioninduced depressive relapse. Arch Gen Psychiatry 54(4): 364-374.

Brown CM, Fletcher PJ, Coscina DV (1998). Acute amino acid loads that deplete brain serotonin fail to alter behavior. Pharmacology, biochemistry, and behavior 59(1): 115-121.

Cahir M, Ardis T, Reynolds GP, Cooper SJ (2007). Acute and chronic tryptophan depletion differentially regulate central 5-HT1A and 5-HT 2A receptor binding in the rat. Psychopharmacology 190(4): 497-506.

Cahir M, Ardis TC, Elliott JJ, Kelly CB, Reynolds GP, Cooper SJ (2008). Acute tryptophan depletion does not alter central or plasma brain-derived neurotrophic factor in the rat. Eur Neuropsychopharmacol 18(5): 317-322.

Carpenter LL, Anderson GM, Pelton GH, Gudin JA, Kirwin PD, Price LH, Heninger GR, McDougle CJ (1998). Tryptophan depletion during continuous CSF sampling in healthy human subjects. Neuropsychopharmacology 19(1): 26-35.

Cohen Z, Bonvento G, Lacombe P, Hamel E (1996). Serotonin in the regulation of brain microcirculation. Prog Neurobiol 50(4): 335-362.

Dawson VL, Dawson TM (1996). Nitric oxide actions in neurochemistry. Neurochem Int 29(2): 97-110.

Dixon WJ (1959). Analysis of extreme values. Ann Math Stat 21: 488-506.

Domek-Lopacinska K, Strosznajder JB (2008). The effect of selective inhibition of cyclic GMP hydrolyzing phosphodiesterases 2 and 5 on learning and memory processes and nitric oxide synthase activity in brain during aging. Brain Res 1216: 68-77. 
Drevets WC, Ongur D, Price JL (1998a). Reduced glucose metabolism in the subgenual prefrontal cortex in unipolar depression. Mol Psychiatry 3(3): 190-191.

Drevets WC, Ongur D, Price JL (1998b). Neuroimaging abnormalities in the subgenual prefrontal cortex: implications for the pathophysiology of familial mood disorders. Mol Psychiatry 3(3): 220-226, 190-191.

Drevets WC, Frank E, Price JC, Kupfer DJ, Holt D, Greer PJ, Huang Y, Gautier C, Mathis C (1999). PET imaging of serotonin 1A receptor binding in depression. Biol Psychiatry 46(10): $1375-1387$.

Drevets WC (2000). Neuroimaging studies of mood disorders. Biol Psychiatry 48(8): 813-829.

Drevets WC (2001). Neuroimaging and neuropathological studies of depression: implications for the cognitive-emotional features of mood disorders. Curr Opin Neurobiol 11(2): 240-249.

Dunn RT, Willis MW, Benson BE, Repella JD, Kimbrell TA, Ketter TA, Speer AM, Osuch EA, Post RM (2005). Preliminary findings of uncoupling of flow and metabolism in unipolar compared with bipolar affective illness and normal controls. Psychiatry Res 140(2): 181-198.

Edvinsson L, MacKenzie ET (1976). Amine mechanisms in the cerebral circulation. Pharmacol Rev 28(4): 275-348.

Evers EA, Tillie DE, van der Veen FM, Lieben CK, Jolles J, Deutz NE, Schmitt JA (2005). Effects of a novel method of acute tryptophan depletion on plasma tryptophan and cognitive performance in healthy volunteers. Psychopharmacology 178(1): 92-99.

Fadda F, Cocco S, Rossetti ZL, Melis G, Stancampiano R (2000a). A tryptophan-free diet markedly reduces frontocortical 5-HT release, but fails to modify ethanol preference in alcohol-preferring (sP) and non-preferring (sNP) rats. Behav Brain Res 108(2): 127-132.

Fadda F, Cocco S, Stancampiano R (2000b). A physiological method to selectively decrease brain serotonin release. Brain Res Brain Res Protoc 5(3): 219-222.

Fernstrom JD, Wurtman RJ (1972). Brain serotonin content: physiological regulation by plasma neutral amino acids. Science 178(59): 414-416.

Fernstrom JD (1979). Diet-induced changes in plasma amino acid pattern: effects on the brain uptake of large neutral amino acids, and on brain serotonin synthesis. J Neural Transm Suppl(15): 55-67.

Fernstrom JD (1983). Role of precursor availability in control of monoamine biosynthesis in brain. Physiol Rev 63(2): 484-546.

Fernstrom JD (1986). Acute and chronic effects of protein and carbohydrate ingestion on brain tryptophan levels and serotonin synthesis. Nutr Rev 44 Suppl: 25-36.

Ferrington L, Kirilly E, McBean DE, Olverman HJ, Bagdy G, Kelly PA (2006). Persistent cerebrovascular effects of MDMA and acute responses to the drug. Eur J Neurosci 24(2): 509-519.

Ford I, McColl JH, McCormack AG, McCrory SJ (1991). Statistical issues in the analysis of neuroimages. J Cereb Blood Flow Metab 11(2): A89-95.

Geiser S, Greenhouse SW (1958). Box's results on the use of the F distribution in multivariate analysis. Ann Math Statist 29(885): 885--891.

Gessa GL, Biggio G, Fadda F, Corsini GU, Tagliamonte A (1974). Effect of the oral administration of tryptophan-free amino acid mixtures on serum tryptophan, brain tryptophan and serotonin metabolism. J Neurochem 22(5): 869-870.

Gessa GL, Biggio G, Fadda F, Corsini GU, Tagliamonte A (1975). Tryptophan-free diet: a new means for rapidly decreasing brain tryptophan content and serotonin synthesis. Acta Vitaminol Enzymol 29(1-6): 72-78.

Grasby PM, Sharp T, Allen T, Kelly PA, Grahame-Smith DG (1992). Effects of the 5-HT1A partial agonists gepirone, ipsapirone and buspirone on local cerebral glucose utilization in the conscious rat. Psychopharmacology (Berl) 106(1): 97-101.

Hood SD, Bell CJ, Nutt DJ (2005). Acute tryptophan depletion. Part I: rationale and methodology. The Australian and New Zealand journal of psychiatry 39(7): 558-564.

Jans LA, Lieben CK, Blokland A (2007a). Influence of sex and estrous cycle on the effects of acute tryptophan depletion induced by a gelatin-based mixture in adult Wistar rats. Neuroscience 147(2): 304-317. 
Jans LA, Riedel WJ, Markus CR, Blokland A (2007b). Serotonergic vulnerability and depression: assumptions, experimental evidence and implications. Mol Psychiatry 12(6): 522-543.

Jans LA, Lieben CK, Smits LT, Blokland A (2008). Pharmacokinetics of acute tryptophan depletion using a gelatin-based protein in male and female Wistar rats. Amino Acids.

Jennrich R, Sampson P, Frane J. 1990. Analysis of variance and covariance with repeated measures. In: BMDP Statistical Software Manual, Vol. 1 (Dixon, W.J., Brown, M.B., Engelman, L., Jenrich R.I.). Berkeley, University of California Press, pp 486-527.

Kelly PA, Davis CJ, Goodwin GM (1988). Differential patterns of local cerebral glucose utilization in response to 5-hydroxytryptamine agonists. Neuroscience 25(3): 907-915.

Kelly PA, Thomas CL, Ritchie IM, Arbuthnott GW (1994). Cerebrovascular autoregulation in response to hypertension induced by NG-nitro-L-arginine methyl ester. Neuroscience 59(1): 13-20.

Kelly PA, Ritchie IM, Arbuthnott GW (1995). Inhibition of neuronal nitric oxide synthase by 7 nitroindazole: effects upon local cerebral blood flow and glucose use in the rat. J Cereb Blood Flow Metab 15(5): 766-773.

Klaassen T, Riedel WJ, van Someren A, Deutz NE, Honig A, van Praag HM (1999). Mood effects of 24-hour tryptophan depletion in healthy first-degree relatives of patients with affective disorders. Biol Psychiatry 46(4): 489-497.

Klaassen T, Riedel WJ, Deutz NE, Van Praag HM (2002). Mood congruent memory bias induced by tryptophan depletion. Psychol Med 32(1): 167-172.

Kuschinsky W (1991). Coupling of function, metabolism, and blood flow in the brain. Neurosurg Rev 14(3): 163-168.

Leonard BE. 1997. Fundamentals of Psychopharmacology. New York: Wiley and Sons.

Lieben CK, Blokland A, Westerink B, Deutz NE (2004a). Acute tryptophan and serotonin depletion using an optimized tryptophan-free protein-carbohydrate mixture in the adult rat. Neurochem Int 44(1): 9-16.

Lieben CK, van Oorsouw K, Deutz NE, Blokland A (2004b). Acute tryptophan depletion induced by a gelatin-based mixture impairs object memory but not affective behavior and spatial learning in the rat. Behav Brain Res 151(1-2): 53-64.

Maes M, Meltzer HY. 1995. The serotonin hypothesis of major depression. E. BF, D.J. K, editors. New York: Raven Press, Ltd. 933-944 p.

Markus CR, Panhuysen G, Tuiten A, Koppeschaar H, Fekkes D, Peters ML (1998). Does carbohydrate-rich, protein-poor food prevent a deterioration of mood and cognitive performance of stress-prone subjects when subjected to a stressful task? Appetite 31(1): 4965.

McBean DE, Sharkey J, Ritchie IM, Kelly PA (1991). Cerebrovascular and functional consequences of 5-HT1A receptor activation. Brain Res 555(1): 159-163.

McCulloch J, Kelly PA, Ford I (1982). Effect of apomorphine on the relationship between local cerebral glucose utilization and local cerebral blood flow (with an appendix on its statistical analysis). J Cereb Blood Flow Metab 2(4): 487-499.

Moja EA, Cipolla P, Castoldi D, Tofanetti O (1989). Dose-response decrease in plasma tryptophan and in brain tryptophan and serotonin after tryptophan-free amino acid mixtures in rats. Life Sci 44(14): 971-976.

Moreno FA, Heninger GR, McGahuey CA, Delgado PL (2000a). Tryptophan depletion and risk of depression relapse: a prospective study of tryptophan depletion as a potential predictor of depressive episodes. Biol Psychiatry 48(4): 327-329.

Moreno FA, McGavin C, Malan TP, Gelenberg AJ, Heninger GR, Mathe AA, Delgado PL (2000b). Tryptophan depletion selectively reduces CSF 5-HT metabolites in healthy young men: results from single lumbar puncture sampling technique. Int J Neuropsychopharmacol 3(4): 277-283.

Neumeister A, Konstantinidis A, Stastny J, Schwarz MJ, Vitouch O, Willeit M, Praschak-Rieder N, Zach J, de Zwaan M, Bondy B, Ackenheil M, Kasper S (2002). Association between serotonin transporter gene promoter polymorphism (5HTTLPR) and behavioral responses 
to tryptophan depletion in healthy women with and without family history of depression. Arch Gen Psychiatry 59(7): 613-620.

Neumeister A (2003). Tryptophan depletion, serotonin, and depression: where do we stand? Psychopharmacol Bull 37(4): 99-115.

Neumeister A, Nugent AC, Waldeck T, Geraci M, Schwarz M, Bonne O, Bain EE, Luckenbaugh DA, Herscovitch P, Charney DS, Drevets WC (2004). Neural and behavioral responses to tryptophan depletion in unmedicated patients with remitted major depressive disorder and controls. Arch Gen Psychiatry 61(8): 765-773.

Neumeister A, Hu XZ, Luckenbaugh DA, Schwarz M, Nugent AC, Bonne O, Herscovitch P, Goldman D, Drevets WC, Charney DS (2006). Differential effects of 5-HTTLPR genotypes on the behavioral and neural responses to tryptophan depletion in patients with major depression and controls. Arch Gen Psychiatry 63(9): 978-986.

Nobler MS, Mann JJ, Sackeim HA (1999). Serotonin, cerebral blood flow, and cerebral metabolic rate in geriatric major depression and normal aging. Brain Res Brain Res Rev 30(3): 250-263.

Olivier JD, Jans LA, Korte-Bouws GA, Korte SM, Deen PM, Cools AR, Ellenbroek BA, Blokland A (2008). Acute tryptophan depletion dose dependently impairs object memory in serotonin transporter knockout rats. Psychopharmacology (Berl) 200(2): 243-254.

Paxinos G, Watson C (1998). The Rat Brain in Stereotaxic Coordinates, 4th ed., vol. 4. Elsevier Academic Press, San Diego. (4th ed).

Prast H, Philippu A (2001). Nitric oxide as modulator of neuronal function. Prog Neurobiol 64(1): 51-68.

Quate L, McBean DE, Ritchie IM, Olverman HJ, Kelly PA (2004). Acute methylenedioxymethamphetamine administration: effects on local cerebral blood flow and glucose utilisation in the Dark Agouti rat. Psychopharmacology (Berl) 173(3-4): 287-925.

Riedel WJ, Klaassen T, Deutz NE, van Someren A, van Praag HM (1999). Tryptophan depletion in normal volunteers produces selective impairment in memory consolidation. Psychopharmacology (Berl) 141(4): 362-369.

Riedel WJ (2004). Cognitive changes after acute tryptophan depletion: what can they tell us? Psychol Med 34(1): 3-8.

Rutten K, Lieben C, Smits L, Blokland A (2007). The PDE4 inhibitor rolipram reverses object memory impairment induced by acute tryptophan depletion in the rat. Psychopharmacology (Berl) 192(2): 275-282.

Sakurada O, Kennedy C, Jehle J, Brown JD, Carbin GL, Sokoloff L (1978). Measurement of local cerebral blood flow with iodo [14C] antipyrine. Am J Physiol 234(1): H59-66.

Sambeth A, Blokland A, Harmer CJ, Kilkens TO, Nathan PJ, Porter RJ, Schmitt JA, Scholtissen B, Sobczak S, Young AH, Riedel WJ (2007). Sex differences in the effect of acute tryptophan depletion on declarative episodic memory: a pooled analysis of nine studies. Neurosci Biobehav Rev 31(4): 516-529.

Sambeth A, Riedel W, Tillie D, Blokland A, Postma A, Schmitt J (2009). Memory impairments in humans after acute tryptophan depletion using a novel gelatin-based protein drink. J Psychopharmacol 23(1): 56-64.

Smith KA, Fairburn CG, Cowen PJ (1997). Relapse of depression after rapid depletion of tryptophan. Lancet 349(9056): 915-919.

Smith KA, Morris JS, Friston KJ, Cowen PJ, Dolan RJ (1999). Brain mechanisms associated with depressive relapse and associated cognitive impairment following acute tryptophan depletion. Br J Psychiatry 174: 525-529.

Soares JC, Mann JJ (1997). The functional neuroanatomy of mood disorders. J Psychiatr Res 31(4): 393-432.

Sokoloff L, Reivich M, Kennedy C, Des Rosiers MH, Patlak CS, Pettigrew KD, Sakurada O, Shinohara M (1977). The [14C]deoxyglucose method for the measurement of local cerebral glucose utilization: theory, procedure, and normal values in the conscious and anesthetized albino rat. J Neurochem 28(5): 897-916.

Sokoloff L (1981a). Relationships among local functional activity, energy metabolism, and blood flow in the central nervous system. Fed Proc 40(8): 2311-2316. 
Sokoloff L (1981b). The deoxyglucose method for the measurement of local glucose utilization and the mapping of local functional activity in the central nervous system. Int Rev Neurobiol 22: 287-333.

Stancampiano R, Melis F, Sarais L, Cocco S, Cugusi C, Fadda F (1997). Acute administration of a tryptophan-free amino acid mixture decreases 5 -HT release in rat hippocampus in vivo. Am J Physiol 272(3 Pt 2): R991-994.

Steinbusch HW (1981). Distribution of serotonin-immunoreactivity in the central nervous system of the rat-cell bodies and terminals. Neuroscience 6(4): 557-618.

Talbot PS, Cooper SJ (2006). Anterior cingulate and subgenual prefrontal blood flow changes following tryptophan depletion in healthy males. Neuropsychopharmacology 31(8): $1757-$ 1767.

van der Plasse G, Meerkerk DT, Lieben CK, Blokland A, Feenstra MG (2007). Lack of evidence for reduced prefrontal cortical serotonin and dopamine efflux after acute tryptophan depletion. Psychopharmacology (Berl) 195(3): 377-385.

van Donkelaar EL, Rutten K, Blokland A, Akkerman S, Steinbusch HW, Prickaerts J (2008). Phosphodiesterase 2 and 5 inhibition attenuates the object memory deficit induced by acute tryptophan depletion. Eur J Pharmacol 600(1-3): 98-104.

van Eijk HM, Rooyakkers DR, Deutz NE (1993). Rapid routine determination of amino acids in plasma by high-performance liquid chromatography with a 2-3 microns Spherisorb ODS II column. J Chromatogr 620(1): 143-148.

Williams WA, Shoaf SE, Hommer D, Rawlings R, Linnoila M (1999). Effects of acute tryptophan depletion on plasma and cerebrospinal fluid tryptophan and 5-hydroxyindoleacetic acid in normal volunteers. J Neurochem 72(4): 1641-1647. 



\title{
Chapter
}

\section{Altered cerebrovascular control following acute tryptophan depletion in MDMA-pretreated rats}

\author{
Eva L. van Donkelaar ${ }^{1}$, Paul A.T. Kelly ${ }^{2}$, Neil Dawson ${ }^{2}$, \\ Arjan Blokland ${ }^{3}$, Jos Prickaerts ${ }^{1}$, Harry W.M. Steinbusch ${ }^{1}$, \\ Linda Ferrington ${ }^{4}$
}

${ }^{1}$ Department of Neuroscience, Faculty of Health, Medicine and Life Sciences, School for Mental Health and Neuroscience, Maastricht University, The Netherlands

${ }^{2}$ Cerebrovascular Research Laboratory, Centre for Cognitive and Neural Systems, University of Edinburgh, United Kingdom

${ }^{3}$ Department of Neuropsychology and Psychopharmacology, Faculty of Psychology and Neuroscience, Maastricht University, The Netherlands

${ }^{4}$ Centre for Cognitive and Neural Systems, University of Edinburgh, United Kingdom

Journal of Neuroscience Research, submitted 



\begin{abstract}
Although 3,4-methylenedioxymethamphetamine (MDMA; 'ecstasy') is known to cause significant serotonergic terminal depletion, reports of depressive symptomatology after repeated recreational use of the drug are controversially. Both depression and MDMA abuse are associated with disturbed serotonin (5-hydroxytryptamine; 5-HT) metabolism and cerebrovascular abnormalities. In the present study, the effects of repeated MDMA pretreatment were measured upon local cerebral blood flow (CBF) and cerebral metabolic rate of glucose (CMRG) in male Wistar rats after an acute tryptophan depletion (ATD) challenge. Three weeks prior to oral administration of a nutritional mixture without tryptophan (TRP), animals were repeatedly treated with MDMA. Subsequently, the effects of ATD upon CBF and CMRG were measured in 43 brain areas using [14C]iodoantipyrine and [14C]-2-deoxyglucose quantitative autoradiography, respectively. MDMA induced significant 5-HT neurotoxicity and ATD resulted in a mean $40 \%$ reduction of the ratio TRP/sum large neutral amino acids in plasma. Global analysis of all brain regions revealed a close $\mathrm{CBF}: \mathrm{CMRG}$ correlation in all groups and a downwards resetting of the relationship of $\mathrm{CBF}$ to CMRG was only observed after ATD treatment in the drug-naïve control animals. This actually indicates that ATD induced a significant upwards resetting of this relationship after MDMA pretreatment compared to controls. These results suggest that ATD produces a degree of cerebrovascular hyperperfusion in animals pretreated with MDMA which could indicate a predisposition to cerebrovascular accidents, in particular haemorrhagic stroke. Moreover, it is tempting to speculate that changes in $\mathrm{CBF}$ might contribute to the behavioural dysfunction of depression, which remains to be further elucidated.
\end{abstract}




\section{Introduction}

3,4-methylenedioxymethamphetamine (MDMA or 'ecstasy') is a semisynthetic entactogen of the phenethylamine family (Nichols and Oberlender, 1990). Acute administration of the psychoactive amphetamine analogue induces a rapid and excessive release of the monoamines norepinephrine $(\mathrm{NE})$, serotonin (5-hydroxytryptamine; 5-HT) and dopamine (DA), of which the latter two are thought to be responsible for its simultaneous hallucinogen-like (serotonergic) and stimulant-like (dopaminergic) effects in humans. The increase in extracellular levels of the monoamines is subsequently maintained and even prolonged due to the inhibition by MDMA of monoaminergic reuptake mechanisms and the monoamine oxidase (MAO) enzyme. In rats and nonhuman primates, repeated administration or high-dose MDMA deactivates the enzyme tryptophan hydroxylase (TPH), thereby inhibiting 5-HT synthesis (Stone et al., 1987). The resulting long-lasting depletion of brain 5-HT causes serious neurotoxicity in the axon terminals of 5-HT neurons (Baumann et al., 2007; Green et al., 2003; Ricaurte et al., 2000), eventually causing a selective loss of 5-HT nerve terminals (Commins et al., 1987; O'Hearn et al., 1988). This 5-HT axon terminal degeneration is reflected in a considerable and persistent decrease in 5-HT transporter (SERT) binding sites (Battaglia et al., 1987; Schmidt, 1987).

Despite the persistent MDMA-induced neurotoxicity, functional impairments in laboratory animals do not parallel the supposed irreversible consequences of the principle alterations in the 5-HT system. This is, however, in keeping with the observation that neurotoxic MDMA exposure rarely causes persistent changes in baseline measures of neural function (Baumann et al., 2007), which in turn might explain why long-term effects reported upon brain functioning in human subjects remain controversial (Guillot, 2007). Any functional deficit might, therefore, remain at a sub-clinical level and only become apparent as a result of challenges to the 5 -HT system. Thus, systemic pharmacological challenges of the 5-HT system have been shown to effectively unmask consequences of MDMA-induced toxicity and additionally provide more insight into its underlying mechanisms (Taffe et al., 2002).

Acute tryptophan depletion (ATD) is a non-pharmacological tool that indirectly challenges the 5-HT system by lowering the plasma levels of its dietary precursor TRP (Bell et al., 2005; for review see: Fadda, 2000; Hood et al., 2005; Neumeister, 2003). Currently, the method represents the most well established human challenge test to investigate the involvement of the 5-HT system in the pathogenesis and pathophysiology of depression. Moreover, ATD has been proposed as a potential tool for predicting subjects at risk of a future depressive episode, as mood-lowering effects have only been reported in healthy subjects with pre-existing abnormal 5-HT functioning (Booij et al., 2002; Booij et al., 2005; Moreno et al., 2000; Van der Does, 2001). The method is non-toxic and thus reversible, thereby allowing the manipulation of the 
central 5-HT system in vivo repeatedly and the assessment of the functional effects of an acute change in 5-HT metabolism in the brain (Fadda et al., $2000 \mathrm{~b}$ ). In human subjects with a history of MDMA abuse, ATD-induced altered TRP metabolism has been associated with persistent memory impairments (Curran and Verheyden, 2003) and altered sensitivity to ATD was found in rhesus monkeys exposed to neurotoxic doses of MDMA (Taffe et al., 2003).

The present study was conducted to investigate possible persistent effects of repeated MDMA administration upon both neuronal and cerebrovascular function in adult brain that might be manifested in altered sensitivity to an acute challenge of the 5-HT system. Therefore, the effects of repeated MDMA pretreatment upon local $\mathrm{CBF}$ and $\mathrm{CMRG}$ were measured in parallel groups of male Wistar rats under an ATD challenged condition, thereby investigating the relationship between cerebrovascular function and 5-HT changes in general and MDMA-induced serotonergic dysfunction as a vulnerability factor for depression and cognitive dysfunction in particular.

\section{Experimental procedures}

\section{Animals}

The principles of laboratory animal care were followed throughout all experiments with particular attention paid to minimizing the number of animals used. All animal procedures were performed with local ethical approval and in accordance with the United Kingdom Animals (Scientific Procedures) Act, 1986 and associated guidelines. A total of 44 male Wistar rats weighing between 150 and $200 \mathrm{~g}$ at the outset were used in this study. Animals were group-housed under a 12-hour light/dark cycle (lights on from 07:00 h - 19:00 h) with access to food and water ad libitum until the night before the experiment. Room temperature in the animal house was maintained at $21 \pm 2^{\circ} \mathrm{C}$.

\section{MDMA pretreatment}

Three weeks prior to the experimental procedures, 21 animals were taken at random from the colony and pretreated with $20 \mathrm{mg} \cdot \mathrm{kg}^{-1}$ of MDMA (( \pm$)-3,4-$ methylenedioxymethamphetamine hydrochloride (Ultrafine Ltd., Manchester, U.K.), twice daily (9:00 AM and 5:00 PM), for 4 consecutive days ( $2 \times 4 \times 20$ mg.kg-1 i.p.). The remaining group of animals $(n=23)$ served as control group. MDMA was dissolved in saline and animals injected with a dose equivalent to $15 \mathrm{mg} \cdot \mathrm{kg}^{-1}$ free base in a volume of $1 \mathrm{ml} . \mathrm{kg}^{-1}$. This specific dosing regime has previously been shown to result in significant decreases of 5-HT, 5-HIAA and 5-HT uptake sites, reflecting a neurotoxicity that is specific to serotonergic systems, which are still in evidence after 6 months (Battaglia et al., 1988). 


\section{Acute tryptophan depletion}

As in previous studies by our group, acute reduction of plasma tryptophan levels was achieved by means of oral administration by gavage of a TRP-free protein-carbohydrate nutritional mixture. (Gelatine hydrolysate, Solugel囚; PB Gelatins, Tessenderlo, Belgium; for amino acid composition see: van Donkelaar et al., 2008). Carbohydrates (Glucodry 210; Amylumgroup, Koog aan de Zaan, The Netherlands) were added to the nutritional mixture to activate the insulin response. A sudden rise in insulin increases protein synthesis, thereby promoting an optimized reduction in plasma TRP levels (Fernstrom, 1986; Lieben et al., 2004a; Markus et al., 1998). Since TRP competes at the bloodbrain barrier with the other large neutral amino acids (LNAAs; leucine [LEU], isoleucine [ILE], phenylalaline [PHE], tyrosine [TYR], valine [VAL]) for the same amino acid L-carrier transport system (Fernstrom and Wurtman, 1972; Fernstrom, 1983), a decrease of the ratio of TRP to the sum of the other LNAAs in plasma (TRP/ $\Sigma$ LNAA) is normally used as the best predictor of reduced central availability of TRP (Fernstrom, 1979, 1983).

Potassium chloride $(\mathrm{KCl})$ and calcium chloride dihydrate $(\mathrm{CaCl} 2.2 \mathrm{H} 2 \mathrm{O})$ were obtained from Merck (Darmstadt, Germany). For the control group, L-tryptophan (Sigma, Zwijndrecht, The Netherlands) was added in an amount equivalent to $0.28 \%$ of total protein content, as adapted from Lieben et al. (2004a). Mixtures were dissolved in distilled water and freshly prepared on the day of the experiment.

For the measurement of the effects of MDMA pretreatment and ATD upon local CBF and CMRG, animals were randomly assigned to the TRP- or TRP+ treatment groups. Surgical procedures to allow the fully quantitative measurement of $\mathrm{CBF}$ and $\mathrm{CMRG}$ were carried out as described elsewhere (Ferrington et al., 2006; Kelly et al., 1995; Quate et al., 2004). One hour following recovery from surgery, animals were orally dosed by gavage, and again after a $90 \mathrm{~min}$ interval (Lieben et al., 2004a). The oral dosing consisted of the nutritional mixture, either with (TRP+ group) or without TRP (TRPgroup), in a volume of $10 \mathrm{ml} / \mathrm{kg}$. Each administration contained $4 \mathrm{~g} / \mathrm{kg}$ bodyweight of Solugel ${ }^{\circledR}$ and $2 \mathrm{~g} / \mathrm{kg}$ Glucodry. The availability of dietary TRP was reduced by removing food from the cages on the night before the experiments. Water was available at all times during the experiments.

Previous studies using the same nutritional mixture (Solugel ${ }^{\circledR}$ ) and dosing regime (Cahir et al., 2007; Jans et al., 2007; Jans et al., 2008b; Lieben et al., 2004a; van der Plasse et al., 2007) have reported significant decreases in both peripheral and central TRP concentrations in male Wistar rats, approximately 4 $\mathrm{h}$ after the first oral administration of the TRP-free nutritional mixture. The measurements of CMRG and CBF were initiated at 220 and $240 \mathrm{~min}$ respectively after the first oral administration by gavage. The median point of integrated specific 2-deoxyglucose in the brain, from which CMRG is derived, is around 10-20 min after an intravenous bolus injection of the tracer (Sokoloff 
et al., 1977) and CBF studies were timed to coincide with this, as previously described (Ferrington et al., 2006; Kelly et al., 1995; Quate et al., 2004).. Mean arterial blood pressure and rectal temperature were monitored throughout the entire experiment.

\section{Measurement of local CMRG}

A total of 10 MDMA pretreated animals (TRP+ $n=4$; TRP- $n=6$ ) and 12 control animals (TRP $+n=6$; TRP- $n=6$ ) were used for the measurement of CMRG, using the fully quantitative $\left[{ }^{14} \mathrm{C}\right]$-2-deoxyglucose autoradiographic imaging technique described previously (Sokoloff, 1981). At $220 \mathrm{~min}$ after the first oral administration of the nutritional mixture, an intravenous injection of $\left[{ }^{14} \mathrm{C}\right]$-2-deoxyglucose (1.3 MBq per rat in $0.75 \mathrm{ml}$ saline; Sigma-Aldrich, UK) was administered at a constant rate over the first $30 \mathrm{~s}$ of the experiment. Over the subsequent $45 \mathrm{~min}$, a total of 14 timed arterial blood samples were collected at pre-determined intervals and centrifuged in order to separate the plasma. Aliquots $(20 \mu \mathrm{l})$ of each plasma sample were taken for the determination of $\left[{ }^{14} \mathrm{C}\right]$ and glucose by liquid scintillation analysis (Packard, Tricarb 2900TL) and semiautomated glucose oxidase enzyme assay (Beckman), respectively. At 45 min, the animals were killed by rapid intravenous injection of sodium pentobarbitone and the brains were immediately dissected out and cut in two. One hemisphere (at random) was rapidly frozen in pre-cooled 2-methylbutane $\left(-45^{\circ} \mathrm{C}\right)$, mounted onto specimen holders with embedding medium (Shanon M-1; Thermo Electron Corp., Pittsburgh, PA, USA) and stored at $-80^{\circ} \mathrm{C}$ until sectioned. The other hemisphere was processed for subsequent amino acid and 5-HT determination.

\section{Measurement of local CBF}

Local cerebral blood flow was measured in a parallel group of animals. A total of $11($ TRP $+n=5$; TRP $-n=6)$ MDMA pretreated and 11 control rats (TRP+ $\mathrm{n}=4$; TRP- $\mathrm{n}=7$ ) were used, using the quantitative $\left[{ }^{14} \mathrm{C}\right]$-iodoantipyrine autoradiographic imaging technique described previously (Sakurada et al., 1978). The $\left[{ }^{14} \mathrm{C}\right]$-iodoantipyrine $(1.5 \mathrm{MBq}$ per rat in $0.6 \mathrm{ml}$ saline; ARC (UK) Ltd, Cardiff, UK) was infused intravenously at a constantly increasing rate over $45 \mathrm{~s}$, at the end of which the animals were killed by decapitation and the brains dissected out and prepared as for the CMRG experiments. During the measurement, a maximum of 18 timed arterial blood samples were collected onto pre-weighed filter paper discs from the freely flowing femoral arterial cannula. Prior to preparation for liquid scintillation analysis, the discs were reweighed, and assuming a specific gravity of 1.05 , the volume of each sample was calculated. 


\section{Preparation and analysis of autoradiograms}

Semi-serial sections $(20 \mu \mathrm{m})$ of the frozen cerebral hemispheres were cut in the coronal plane in a cryostat $\left(-22^{\circ} \mathrm{C}\right)$. A series of three sections were retained from every $200 \mu \mathrm{m}$, thaw mounted onto a glass coverslip and rapidly dried on a hot plate maintained at $75^{\circ} \mathrm{C}$. Autoradiograms were prepared by applying these sections to X-ray film (Kodak, SB-5), together with a series of eight precalibrated standards of known $\left[{ }^{14} \mathrm{C}\right]$ concentration $(40-1069 \mathrm{nCi} / \mathrm{g}$ tissue equivalents: Amersham International, U.K.). Films were stored in a light-tight cassette at $4^{\circ} \mathrm{C}$ for 7 days after which they were developed according to the manufacturers instructions.

Analysis of the autoradiograms was performed using a computer-based image analysis system (MCID/M5+). Local tissue isotope concentrations were derived from the optical density of autoradiographic brain images relative to the $\left[{ }^{14} \mathrm{C}\right]$ standards, and $\mathrm{CMRG}$ and $\mathrm{CBF}$ were calculated from tissue $\left[{ }^{14} \mathrm{C}\right]$ concentrations and arterial tracer profiles using the appropriate operational equations. Measurements were made in a total of 43 anatomically distinct and functionally diverse brain regions identified with reference to a rat stereotaxic atlas (Paxinos and Watson, 1998).

\section{Determination of serotonergic lesion}

Three weeks after the repeated MDMA dosing regime, 5-HT terminal depletion was determined by radioligand binding autoradiography of $\left[{ }^{3} \mathrm{H}\right]$-paroxetine binding to 5-HT transporter sites as described by De Souza and Kuyatt (1987a). These transporter sites are localized to 5-HT terminals and MDMA-induced long-term reductions in radioligand binding have been shown to correlate with anatomical evidence of terminal depletion (Battaglia et al., 1987). Sections at 5 different levels throughout the brain of MDMA-pretreated and control animals were thaw-mounted onto gelatin covered glass slides and stored at $-80^{\circ} \mathrm{C}$ for subsequent $\left[{ }^{3} \mathrm{H}\right]$-paroxetine autoradiographic binding analysis of 5 -HT uptake sites. Procedures were carried out according to the protocol described by De Souza and Kuyatt (1987a), with the addition of two 5 minute prewashes in buffer $\left(50 \mathrm{mM}\right.$ TRIS-HCl, $120 \mathrm{mM} \mathrm{NaCl}, 5 \mathrm{mM} \mathrm{KCl}, \mathrm{pH}=7.7$ at $\left.23^{\circ} \mathrm{C}\right)$ to remove $\left[{ }^{14} \mathrm{C}\right]$-tracers from the tissue (De Souza and Kuyatt, 1987b; Sharkey et al., 1991b). Sections were then incubated in the same buffer containing a saturating concentration $(250 \mathrm{pM})$ of $\left[{ }^{3} \mathrm{H}\right]$-paroxetine (specific activity 23.1 $\mathrm{Ci} / \mathrm{mmol}$; New England Nuclear). Non-specific binding was defined in adjacent sections by $\left[{ }^{3} \mathrm{H}\right]$-paroxetine binding in the presence of $4 \mu \mathrm{M}$ citalopram (Tocris Bioscience, Bristol, UK). Following incubation, the sections were washed in buffer, dipped in deionised water and rapidly dried under a stream of cold air. The slides, together with a set of $\left[{ }^{3} \mathrm{H}\right]$-containing standards (Amersham Microscales) were apposed to x-ray film (Amersham Hyperfilm) in a light-tight cassette and stored at $-80^{\circ} \mathrm{C}$ for $4-6$ weeks. Films were further processed 
according to the manufacture's instructions and analysis of the autoradiographic images was performed as described above. In this case, local tissue isotope concentrations were derived from the optical density of the images relative to appropriate $\left[{ }^{3} \mathrm{H}\right]$ - containing standards. Radioligand binding was subsequently calculated from the tissue concentrations and the specific activity of the $\left[{ }^{3} \mathrm{H}\right]$-paroxetine.

\section{Blood collection and preparation for amino acid analysis}

Prior to the first gavage, a blood sample (BS) was taken for determination of baseline amino acid levels in plasma (BSO; 0 ) and a second blood sample (BS1; t1) was taken at the end of the experiment, immediately prior to sacrifice, i.e. at 240 and $265 \mathrm{~min}$ after the first oral administration by gavage for the CBF and CMRG measurements, respectively. For each blood sample, approximately 300 $\mu$ of venous blood was withdrawn via the femoral cannula and collected in sodium heparin coated tubes (Microvette ${ }^{\circledR}$ CB 300, Sarstedt, Germany). The sample tubes were kept on ice until centrifuged for $10 \mathrm{~min}(14,000 \mathrm{x} \mathrm{g})$ in order to separate the plasma. Aliquots of plasma were removed and stored at $-80{ }^{\circ} \mathrm{C}$ for subsequent amino acid determination.

Immediately prior to analysis, plasma samples were thawed to $4{ }^{\circ} \mathrm{C}$ and deproteinized in cups containing $\left(17 \mu \mathrm{l}\right.$ of $\left.0.5 \mathrm{mg} \cdot \mathrm{ml}^{-1}\right)$ dry 5 -sulfosalicylic acid (SSA; Merck, Darmstadt, Germany). Samples were vigorously vortex-mixed and then centrifuged for $10 \mathrm{~min}(50,000 \mathrm{x} \mathrm{g})$. From the clear supernatant a $20 \mu \mathrm{l}$ aliquot was mixed with $1960 \mu \mathrm{l}$ distilled water to which $20 \mu$ l norvaline had been added, and stored in a temperature-maintained $\left(\begin{array}{lll}7 & { }^{\circ} \mathrm{C}\end{array}\right)$ sample compartment until high-performance liquid chromatography (HPLC) analysis.

\section{Brain tissue collection and preparation for amino acid and 5-HT analysis}

From the brain hemispheres collected at the end of the CBF and CMRG measurements, prefrontal cortex and hippocampus were dissected out onto dry ice before storage at $-80^{\circ} \mathrm{C}$. For central amino acid determination, the frozen brain tissue samples were transferred into pre-weighed $2 \mathrm{ml} \mathrm{screw}$-lid cups and exact tissue weight was determined with an analytical balance. Approximately $0.3 \mathrm{~g}$ of glass beads ( $1 \mathrm{~mm}$ diameter) were added to the cup together with icecold $150 \mathrm{mM} \mathrm{H}_{3} \mathrm{PO}_{4}$ and $500 \mu \mathrm{M}$ diethylene triamine pentaacetic acid. Samples were aerated with Argon and homogenized in a mini-bead beater set at high speed ( 3 × $30 \mathrm{~s}$ with cooling in between runs). After a subsequent 3 min spin $\left(4000 \mathrm{x} g\right.$ at $\left.4^{\circ} \mathrm{C}\right)$, the homogenate was transferred back into an Eppendorf vial and centrifuged for a further $20 \mathrm{~min}\left(35,000 \times \mathrm{g}\right.$ at $\left.4^{\circ} \mathrm{C}\right)$. The resulting supernatant was filtered through Millipore Ultrafree-MC filter cups for 1-2 hr $\left(9000 \times \mathrm{g}\right.$ at $\left.4^{\circ} \mathrm{C}\right)$. Finally, $15 \mu \mathrm{l}$ of $2 \mathrm{mM}$ ascorbic acid was added to further 
protect against oxidative degradation processes, after which the samples were stored at $-80^{\circ} \mathrm{C}$ until HPLC analyses.

Plasma and brain amino acid concentrations were quantified using a fully automated HPLC system after pre-column derivatization with o-phthaldialdehyde (Domek-Lopacinska and Strosznajder; van Eijk et al., 1993). Fluorescence detection was used to quantify OPA-AA derivates of the amino acids. Central concentrations of 5-HT and the 5-HT metabolite (5-hydroxyindoleacetic acid; 5-HIAA) in hippocampus and prefrontal cortex were quantified in the remaining supernatant at a later stage by automated HPLC with dual electrochemical detection. The concentrations of total plasma and central amino acid concentrations were expressed as $\mu \mathrm{mol} . \mathrm{L}^{-1}$, and brain concentrations as ng. $\mathrm{mg}^{-1}$.

\section{Statistical analysis}

The mean concentrations of all absolute plasma LNAAs, were determined separately for each experimental condition and each time-point and TRP/ $/$ LNAA ratios were calculated accordingly. Plasma amino acid concentrations were measured both prior to the first oral administration (baseline; $t 0$ ) and at the end of the experiments ( $t 1)$. Since no differences were found in both the MDMA pretreatment group and the control group between either plasma or brain biochemical results at $\mathrm{t} 1=240 \mathrm{~min}(\mathrm{CBF})$ and at $\mathrm{t} 1=$ 265 min (CMRG) after the first oral administration, data in each group were pooled to increase statistical power.

Changes in the ratio TRP $/ \Sigma$ LNAA over time were analyzed by means of repeated measures analysis of variance (ANOVA) with Pretreatment and ATD Treatment as between-subject factors and Time as within-subject variable. To further analyze pretreatment and ATD treatment effects, two-way ANOVAs were performed for each time-point separately. Central TRP, 5-HT, 5-HIAA concentrations and the 5-HT turnover (5-HIAA/5-HT), were analyzed for hippocampus and prefrontal cortex separately by means of two-way ANOVA with Pretreatment and ATD Treatment as between subject factors. Post hoc testing with Bonferroni correction was used wherever appropriate. Extreme values in the plasma and brain biochemical data were calculated using Dixon's Extremity test (Dixon, 1959) and excluded from statistical analysis. Analyses were performed using the Statistical Package for Social Science (SPSS) for Windows (Rel.12.0.1.2003, Chicago: SPSS Inc.).

Local CBF and CMRG data were analyzed by two-way ANOVA with Pretreatment and ATD treatment as between subject factors. To provide an initial assessment of differences between treatment groups in the relationship of local $\mathrm{CBF}$ to local CMRG in each of 43 regions of interest (ROI) included in this study, the ratios of mean local CBF to mean local CMRG derived from each structure were analyzed by Mann-Whitney $U$ test using the curve fitting program GraphPad Prism, version 4.02 for Windows (GraphPad Software, San 
Diego California USA). More rigorous statistical analysis of the relationship was performed by repeated measures ANOVA on the log transform of the data sets (McCulloch et al., 1982) with Measurement (CBF or CMRG) as the betweensubject factor, and "brain region" as within-subject variable. This analysis was performed using the BMDP/PC90 computerized statistical package (program 2V) (Jennrich et al., 1990). While the experimental design was insufficient to allow a full multivariate profile analysis, inter-regional differences in the magnitude of the ratio of local CBF to local CMRG could be examined using an Univariate approach (Ford et al., 1991; Geiser and Greenhouse, 1958).

Data from radioligand binding were analyzed using unpaired Student's $t$-test for grouped data. All data are represented as mean \pm standard error of the mean (S.E.M.) and acceptable levels of significance were set at $P<0.05$.

\section{Results}

\section{Physiological parameters}

There were no significant differences in physiological parameters, either between pretreatment and ATD treatment groups, within ATD treatment groups (before and after treatment), or between animals with similar treatments used for either CBF or CMRG experiments. In particular, there were no significant differences in plasma glucose levels between the MDMA pretreatment group and control animals $\left(1.45 \pm 0.16 \mathrm{mg} \cdot \mathrm{ml}^{-1}\right.$ and $1.48 \pm 0.12$ mg. $\left.\mathrm{ml}^{-1}\right)$ or within the groups before ATD treatment $\left(1.49 \pm 0.16 \mathrm{mg} \cdot \mathrm{ml}^{-1}\right.$ and $1.52 \pm 0.13 \mathrm{mg} \cdot \mathrm{ml}^{-1}$ in TRP- and TRP + groups, respectively) or at the end of the experiment $\left(1.51 \pm 0.21 \mathrm{mg} \cdot \mathrm{ml}^{-1}\right.$ and $1.49 \pm 0.17 \mathrm{mg} \cdot \mathrm{ml}^{-1}$ in TRP- and TRP+ groups, respectively). Thus, plasma glucose levels remained steady throughout the measurement periods.

\section{Plasma amino acid concentrations}

The TRP/ $L \mathrm{LNAA}$ ratio in plasma (Fig. 1) significantly decreased over time $[F(1,37)=58.82, P<0.001]$ depending upon TRP- or TRP+ treatment condition $[F(1,37)=28.27, P<0.001]$. A significant ATD treatment effect indicated that the TRP/ $\mathrm{LNAA}$ ratio was lower after TRP- compared to TRP+ control treatment [ATD treatment: $F(1,37)=13.40, P<0.01]$. In general, higher TRP $/ \Sigma L N A A$ levels were found in the MDMA pretreated group [Pretreatment: $F(1,37)=7.58, P<0.01$ ] after both TRP- and TRP+ treatment $[F(1,37)=0.66$, n.s.]. Separate analysis per time-point revealed no group differences at baseline $(\mathrm{t} 0)[F(1,44)=1.25$, n.s.]. At $\mathrm{t} 1$, the ratio TRP/ $\Sigma$ LNAA was significantly lower after ATD compared to TRP+ control treatment $[F(1,40)=44.27, P<0.01]$ and post hoc analyses revealed that this 
decrease was found in both pretreatment conditions (MDMA pretreatment $-37 \%$, controls: $-40 \%$ ). At this time-point, the levels of the ratio TRP/ $\Sigma$ LNAA were significantly higher after MDMA pretreatment compared to the control pretreatment condition $[F(1,40)=44.27, P<0.01]$, however, post hoc analyses revealed that this was only the case in the TRP+ group. Thus, MDMA pretreatment does not alter the effect of ATD upon the ratio TRP/ $L$ LNAA in plasma.

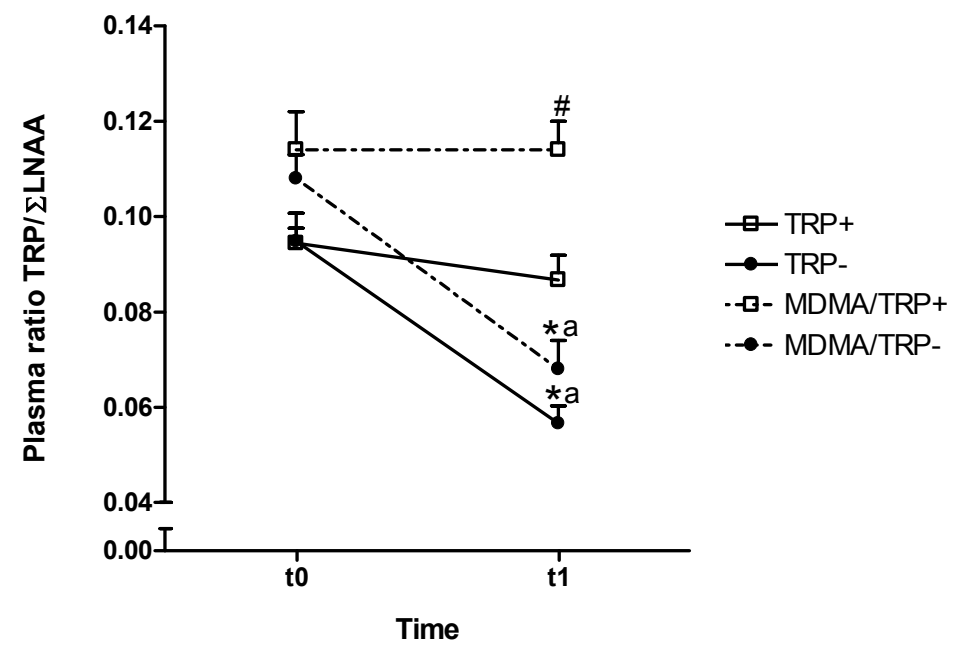

Figure 1 The effect of ATD (TRP-) upon the ratio TRP/ LLNAA in plasma (mean + S.E.M.) at baseline $(\mathrm{t} 0)$ and 240-265 min after the first oral administration ( $\mathrm{t} 1), 3$ weeks after repeated MDMA pretreatment compared to control animals. $\left(^{*}\right)$ significantly different from TRP+ control treatment; $\left(^{\#}\right)$ significantly different from control pretreatment condition; (a) significantly different over time $(*, \#$, a) $P<0.05$.

\section{Brain TRP}

ATD did not affect the TRP levels (Fig. 2A) in the hippocampus $[F(1,18)=$ 0.04 , n.s.], yet TRP concentrations appeared to be generally higher in the MDMA pretreatment group $[F(1,18)=178.41, P<0.001]$. This main effect of Pretreatment would appear to be independent of ATD treatment condition, as no Pretreatment x ATD Treatment interaction was found $[F(1,18)=0.070$, n.s.]. As in the hippocampus, ATD did not alter the TRP levels in the prefrontal cortex $[F(1,33)=1.48$, n.s.]. However, in contrast to the pretreatment effect in the hippocampus, lower TRP levels in the prefrontal cortex were found in the MDMA pretreatment group $[F(1,33)=52.67$, $P<0.001]$, independent of ATD treatment $[F(1,33)=0.54$, n.s.]. 


\section{Brain 5-HT}

ATD did not affect 5-HT concentrations (Fig. 2B) in the hippocampus $[F(1$, $17)=1.45$, n.s.]. However, the concentrations were significantly lower in MDMA pretreated animals $[F(1,17)=186.93, P<0.001]$ after both TRP- and TRP+ treatment $[F(1,17)=0.334$, n.s.]. In the prefrontal cortex, 5-HT levels appeared higher after ATD compared to TRP+ treatment, although no main effect of ATD Treatment was found $[F(1,26)=0.62$, n.s.]. As in the hippocampus, lower 5-HT concentrations were found in the MDMA pretreated animals $[F(1,26)=40.51, P<0.001]$, again independent of ATD treatment condition $[F(1,26)=1.20$, n.s. $]$.

\section{Brain 5-HIAA}

No main effect of ATD Treatment upon 5-HIAA levels (Fig. 2C) was found in the hippocampus $[F(1,20)=0.31$, n.s.]. In the MDMA pretreated animals, the concentration of 5-HIAA was higher compared to the control animals $[F(1,20)$ $=14.79, P<0.01]$. This Pretreatment effect may be supposed to be independent of ATD treatment as no Pretreatment $\mathrm{x}$ ATD treatment interaction was found $[F(1,20)=0.34$, n.s. $]$. However, post hoc analyses revealed that higher hippocampal 5-HIAA concentrations in MDMA pretreated animals were only found after TRP- treatment, not after TRP+ control treatment. In the prefrontal cortex, ATD did not alter the 5-HIAA concentrations $[F(1,33)=$ 0.01 , n.s.]. Moreover, the concentrations appeared to be similar in both pretreatment conditions $[F(1,33)=0.16$, n.s.], independent of ATD treatment $[F(1,33)=0.48$, n.s. $]$.

\section{5-HT turnover}

ATD did not affect the 5-HIAA/5-HT ratio (Fig. 2D) in hippocampus $[F(1$, $17)=0.05$, n.s.], however, $5-\mathrm{HT}$ turnover appeared to be much higher in MDMA pretreated animals compared to controls $[F(1,17)=13.17, P<0.01]$, independent of ATD treatment $[F(1,17)=0.05$, n.s. $]$. A similar pattern was found in the prefrontal cortex, with no main effect of ATD Treatment $[F(1,20$ $=0.06$, n.s. $]$, a significantly higher ratio in the MDMA pretreated group $[F(1$, $20)=8.38, P<0.01]$, and no Pretreatment $\mathrm{x}$ ATD Treatment interaction $[F(1$, 20) $=0.05$, n.s.]. 

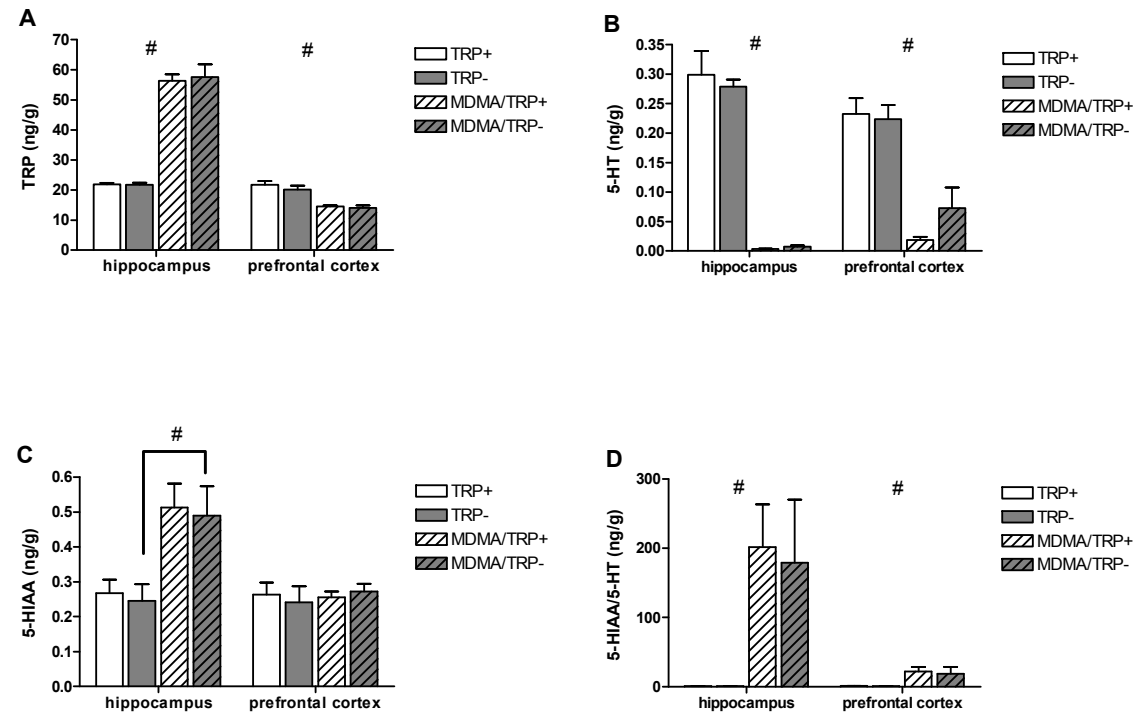

Figure 2 The effect of ATD upon central concentrations of, (A) TRP, (B) 5-HT and (C) 5-HIAA and (D) 5-HT turnover in hippocampus and prefrontal cortex (mean + S.E.M.), 240-265 min after the first oral administration (t1), 3 weeks after repeated MDMA pretreatment compared to control animals. $(\#)$ significantly different from control pretreatment condition. $\left.{ }^{\#}\right) P<0.05$.

\section{Radioligand binding}

Three weeks after the repeated MDMA administration regime $\left[{ }^{3} \mathrm{H}\right]$-Paroxetine binding to 5-HT transporter sites in frontal cortex was significantly reduced $(-45 \%)$ compared to control animals $[t(6)=3.39 ; P<0.05]$. A $57 \%$ reduction was found in the dorsal medial prefrontal cortex $[t(6)=2.53 ; P<0.05]$ while the greatest reductions $(-96 \%)$ were found in the anterior cingulate cortex $[t(9)=$ 4.26; $P<0.05]$. Hippocampal subfields were also significantly affected, with an $86 \%$ decrease in binding found in CA1 [ $t(9)=4.86 ; P<0.05]$, a $92 \%$ decrease in CA2 $[t(9)=5.89 ; P<0.05]$ and a $79 \%$ decrease in CA3 regions $[t(9)=5.17$; $P<0.05]$. No significant changes in binding were found in the entorhinal cortex or dentate gyrus, and the dorsal, median and paramedian raphe also remained unaffected by the repeated MDMA administration regime (Fig. 3).

\section{Local cerebral metabolic rate of glucose}

A significant main effect of MDMA Pretreatment upon local CMRG was observed in 12 out of the 43 ROIs analyzed (Table 1). In three areas, the dorsal medial prefrontal cortex, bed nucleus of the striae terminals and the lateral amygdala this was due to increased CMRG in MDMA pretreated animals compared to controls. In the other nine brain areas, however, the main effect of Pretreatment was the result of decreases in CMRG. In both cases (increases 
or decreases) the MDMA Pretreatment effects were not altered by subsequent ATD treatment and in general, no significant main effect of ATD Treatment upon CMRG was found.

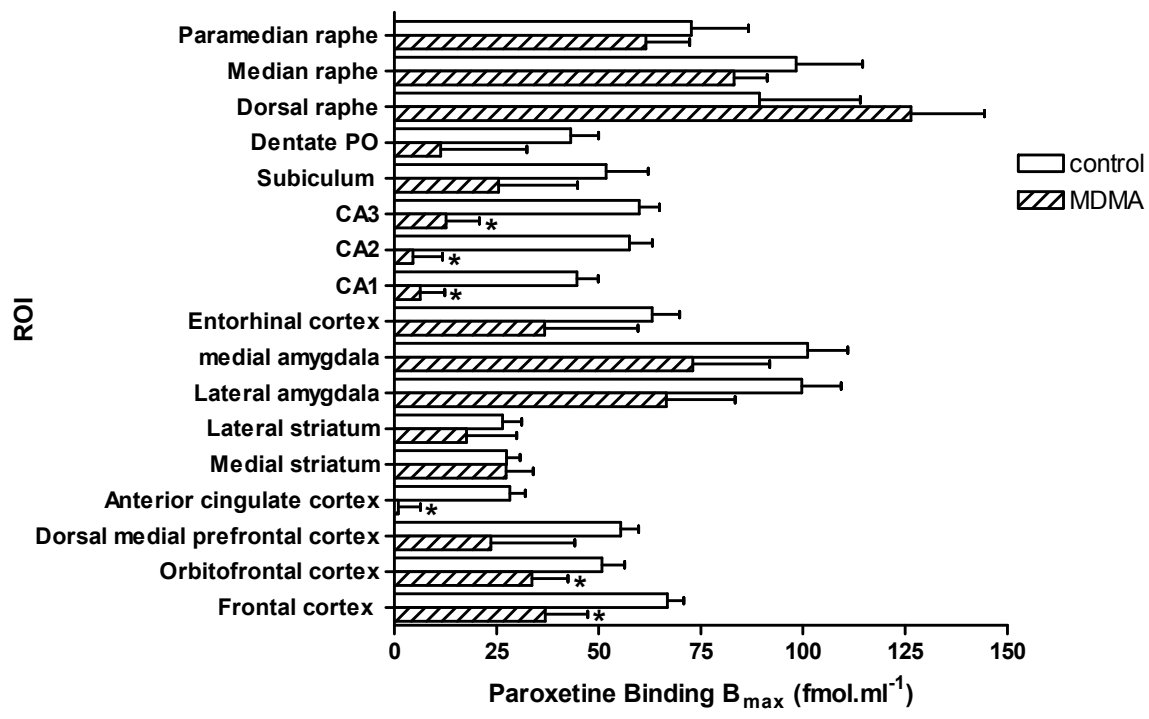

Figure $3[3 \mathrm{H}]$-Paroxetine binding (mean + S.E.M.), 3 weeks after repeated MDMA pretreatment $(n=5)$ compared to saline control rats $(n=6)$. Data represent the $[3 \mathrm{H}]$-paroxetine specific binding $\mathrm{Bmax}\left(\mathrm{fmol} / \mathrm{mg}\right.$ tissue) measured in each of the different brain areas. $\left(^{*}\right) P<0.05$; significant decrease from control animals.

Table 1 Local cerebral metabolic rate of glucose (CMRG, $\mu \mathrm{mol} / 100 \mathrm{~g} / \mathrm{min})$ at $\mathrm{t} 1 \mathrm{after}$ TRP+ treatment or ATD (TRP-) in MDMA pretreated and control rats. Data are represented as mean \pm S.E.M.

\begin{tabular}{lcrcrl}
\hline Brain regions & \multicolumn{2}{l}{ CMRG } & & \multicolumn{2}{l}{ Two-way ANOVA } \\
\cline { 2 - 6 } & \multicolumn{1}{c}{ control } & \multicolumn{2}{c}{ MDMA } & \multicolumn{2}{l}{ P values } \\
\cline { 2 - 6 } & \multicolumn{1}{c}{ TRP+ } & \multicolumn{1}{c}{ TRP- } & TRP + & \multicolumn{1}{c}{ TRP- } & Pretreat ATD Interaction \\
\hline Frontal cortex & $106 \pm 12$ & $98 \pm 5$ & $93 \pm 3$ & $91 \pm 7$ & \\
Orbital frontal cortex & $142 \pm 16$ & $134 \pm 8$ & $123 \pm 2$ & $113 \pm 4$ & \\
Dorsal medial prefrontal cortex & $75 \pm 8$ & $76 \pm 3$ & $103 \pm 3$ & $96 \pm 10$ & 0.01 \\
Ventral medial prefrontal cortex & $109 \pm 12$ & $109 \pm 7$ & $77 \pm 3$ & $73 \pm 6$ & 0.00 \\
Nucleus accumbens & $88 \pm 9$ & $84 \pm 3$ & $86 \pm 3$ & $92 \pm 6$ & \\
Corpus callosum & $22 \pm 3$ & $19 \pm 7$ & $24 \pm 1$ & $24 \pm 3$ & \\
Somatosensory cortex & $119 \pm 10$ & $113 \pm 6$ & $90 \pm 2$ & $99 \pm 7$ & 0.02 \\
Anterior cingulate cortex & $99 \pm 10$ & $98 \pm 5$ & $91 \pm 3$ & $89 \pm 7$ & \\
Septal nucleus & $61 \pm 7$ & $55 \pm 4$ & $59 \pm 6$ & $59 \pm 4$ & \\
Medial striatum & $94 \pm 9$ & $92 \pm 6$ & $80 \pm 2$ & $83 \pm 6$ & \\
Lateral striatum & $106 \pm 11$ & $106 \pm 5$ & $88 \pm 1$ & $89 \pm 8$ & 0.04 \\
\hline
\end{tabular}




\begin{tabular}{|c|c|c|c|c|c|}
\hline \multirow[t]{3}{*}{ Brain regions } & \multicolumn{4}{|l|}{ CMRG } & \multirow{3}{*}{$\begin{array}{l}\text { Two-way ANOVA } \\
P \text { values } \\
\text { Pretreat ATD Interaction }\end{array}$} \\
\hline & \multicolumn{2}{|l|}{ control } & \multicolumn{2}{|l|}{ MDMA } & \\
\hline & TRP+ & TRP- & TRP+ & TRP- & \\
\hline Bed nucleus of striae terminalis & $46 \pm 5$ & $45 \pm 4$ & $61 \pm 2$ & $68 \pm 4$ & 0.00 \\
\hline Globus pallidus & $54 \pm 7$ & $50 \pm 3$ & $45 \pm 1$ & $48 \pm 3$ & \\
\hline Antero ventral thalamic nucleus & $105 \pm 11$ & $97 \pm 12$ & $84 \pm 1$ & $92 \pm 7$ & \\
\hline Antero hypothalamic nucleus & $53 \pm 7$ & $58 \pm 9$ & $51 \pm 2$ & $56 \pm 5$ & \\
\hline Tempero parietal cortex & $114 \pm 11$ & $109 \pm 5$ & $85 \pm 5$ & $79 \pm 2$ & 0.00 \\
\hline Ventral lateral hypothalamic nucleus & $62 \pm 7$ & $52 \pm 3$ & $55 \pm 3$ & $51 \pm 3$ & \\
\hline Medial thalamic nucleus & $100 \pm 8$ & $95 \pm 3$ & $85 \pm 4$ & $79 \pm 5$ & 0.02 \\
\hline Ventral lateral thalamic nucleus & $88 \pm 9$ & $83 \pm 4$ & $79 \pm 6$ & $75 \pm 6$ & \\
\hline Medial amygdala & $84 \pm 10$ & $79 \pm 5$ & $48 \pm 1$ & $52 \pm 6$ & 0.00 \\
\hline Lateral amygdala & $52 \pm 5$ & $49 \pm 2$ & $74 \pm 1$ & $66 \pm 6$ & 0.00 \\
\hline Posterior cingulate cortex & $105 \pm 10$ & $94 \pm 5$ & $89 \pm 3$ & $88 \pm 7$ & \\
\hline Lateral habenula & $116 \pm 15$ & $108 \pm 6$ & $94 \pm 3$ & $85 \pm 3$ & 0.03 \\
\hline Subthalamic nucleus & $84 \pm 7$ & $87 \pm 3$ & $79 \pm 3$ & $78 \pm 4$ & \\
\hline Mamillary body & $110 \pm 12$ & $96 \pm 9$ & $102 \pm 0$ & $94 \pm 6$ & \\
\hline Piriform cortex & $67 \pm 8$ & $60 \pm 3$ & $63 \pm 5$ & $54 \pm 2$ & \\
\hline Entorhinal cortex & $69 \pm 9$ & $62 \pm 2$ & $72 \pm 3$ & $68 \pm 2$ & \\
\hline Molecular layer & $90 \pm 12$ & $84 \pm 5$ & $79 \pm 3$ & $75 \pm 4$ & \\
\hline Dorsal subiculum & $87 \pm 9$ & $86 \pm 5$ & $70 \pm 3$ & $67 \pm 5$ & 0.01 \\
\hline Dentate PO & $56 \pm 7$ & $55 \pm 4$ & $58 \pm 2$ & $57 \pm 4$ & \\
\hline Dorsal CA1 & $67 \pm 9$ & $61 \pm 5$ & $59 \pm 1$ & $60 \pm 3$ & \\
\hline CA2 & $65 \pm 10$ & $66 \pm 5$ & $60 \pm 4$ & $56 \pm 4$ & \\
\hline Ventral CA1 & $75 \pm 10$ & $64 \pm 4$ & $68 \pm 6$ & $62 \pm 3$ & \\
\hline Ventral subiculum & $69 \pm 7$ & $64 \pm 3$ & $64 \pm 5$ & $56 \pm 4$ & \\
\hline $\mathrm{CA} 3$ & $71 \pm 9$ & $70 \pm 3$ & $66 \pm 2$ & $65 \pm 4$ & \\
\hline Ventral tegmental area & $63 \pm 9$ & $61 \pm 6$ & $53 \pm 2$ & $46 \pm 3$ & \\
\hline Substantia nigra reticulata & $59 \pm 6$ & $54 \pm 3$ & $51 \pm 3$ & $46 \pm 2$ & \\
\hline Substantia nigra compacta & $76 \pm 10$ & $72 \pm 4$ & $69 \pm 2$ & $63 \pm 5$ & \\
\hline Dorsal raphe & $74 \pm 9$ & $80 \pm 6$ & $72 \pm 11$ & $71 \pm 4$ & \\
\hline Median raphe & $83 \pm 8$ & $75 \pm 9$ & $73 \pm 9$ & $71 \pm 4$ & \\
\hline Paramedian raphe & $71 \pm 11$ & $64 \pm 7$ & $66 \pm 9$ & $61 \pm 4$ & \\
\hline Inferior colliculus & $203 \pm 26$ & $178 \pm 5$ & $167 \pm 3$ & $148 \pm 10$ & 0.03 \\
\hline Locus coeruleus & $77 \pm 9$ & $64 \pm 4$ & $70 \pm 11$ & $78 \pm 4$ & \\
\hline
\end{tabular}

Where effects are significant $(P<0.05)$, levels of significance generated by ANOVA are provided in columns designated Pretreat (main effect of MDMA Pretreatment); ATD (main effect of ATD Treatment); Interaction (Pretreatment x ATD effect).

\section{Local cerebral blood flow}

A significant main effect of MDMA pretreatment was also found in the effects upon CBF (Table 2). Evidence for increased CBF in MDMA pretreated animals was found not only in those areas where CMRG was increased (dorsal medial prefrontal cortex, bed nucleus of the striae terminals and lateral amygdalae) but 
also in other brain regions, such as septal nucleus, substantia nigra compacta and locus coeruleus. While a significant main effect of ATD treatment was only found in the entorhinal cortex and molecular layer, there was evidence of a significant interaction between MDMA pretreatment and acute ATD (see Table 2 , Interaction).

\section{Relationship between CBF and CMRG}

Global analysis of all ROIs revealed a close correlation between CBF and CMRG after TRP+ and TRP- treatment in both the MDMA pretreated $(r=$ 0.88 and 0.87 , respectively) and control animals ( $r=0.93$ and 0.91 , respectively). A significant increase in the global ratio between blood flow and glucose use was found after ATD in the MDMA pretreated animals when compared to controls as illustrated by a shift in the slope of the best-fitting straight line from 1.07 in controls to 1.48 in MDMA pretreated animals $(P<0.01$; Fig. 4). In contrast, there was no such difference following TRP+ administration in MDMA pretreated animals compared to controls $(P=0.36)$. Examining the brain areas individually, the flow-metabolism ratio was found to decrease in all but 4 of the 43 brain areas in ATD-treated rats (data not shown) and over the brain as a whole analyses of the ratios (Mann-Whitney U-test) indicated a significant decrease in the ATD group (mean ratio $=1.15$ ) when compared to controls (mean ratio $=1.33$ ). These data suggest that ATD produces a degree of cerebrovascular hyperperfusion in animals pretreated with MDMA.

Table 2 Local cerebral blood flow (CBF, $\mathrm{ml} / 100 \mathrm{~g} / \mathrm{min})$ at $\mathrm{t} 1$ after TRP + treatment or ATD (TRP-) in MDMA pretreated and control rats. Data are represented as mean \pm S.E.M.

\begin{tabular}{llllll}
\hline Brain regions & CBF & & & \multicolumn{2}{c}{ Two-way ANOVA } \\
\cline { 2 - 6 } & control & \multicolumn{3}{l}{ MDMA } & \multicolumn{1}{c}{ Pvalues } \\
\cline { 2 - 6 } & TRP+ & TRP- & TRP+ & TRP- & Pretreat ATD Interaction \\
\hline Frontal cortex & $109 \pm 16$ & $89 \pm 8$ & $120 \pm 9$ & $114 \pm 4$ & \\
Orbital frontal cortex & $168 \pm 18$ & $143 \pm 13$ & $149 \pm 14$ & $156 \pm 17$ & \\
Dorsal medial prefrontal cortex & $95 \pm 10$ & $78 \pm 7$ & $116 \pm 9$ & $116 \pm 6$ & 0.00 \\
Ventral medial prefrontal cortex & $125 \pm 16$ & $99 \pm 14$ & $90 \pm 8$ & $91 \pm 3$ & \\
Nucleus accumbens & $121 \pm 14$ & $104 \pm 9$ & $133 \pm 12$ & $122 \pm 7$ & \\
Corpus callosum & $33 \pm 5$ & $28 \pm 1$ & $27 \pm 2$ & $32 \pm 4$ & \\
Somatosensory cortex & $151 \pm 29$ & $114 \pm 7$ & $143 \pm 17$ & $146 \pm 8$ & \\
Anterior cingulate cortex & $117 \pm 17$ & $95 \pm 7$ & $126 \pm 19$ & $123 \pm 6$ & \\
Septal nucleus & $74 \pm 11$ & $66 \pm 4$ & $83 \pm 10$ & $88 \pm 5$ & 0.03 \\
Medial striatum & $99 \pm 13$ & $87 \pm 4$ & $91 \pm 7$ & $100 \pm 9$ & \\
Lateral striatum & $111 \pm 14$ & $95 \pm 5$ & $115 \pm 8$ & $118 \pm 9$ & \\
Bed nucleus of striae terminalis & $56 \pm 5$ & $48 \pm 3$ & $76 \pm 9$ & $88 \pm 6$ & 0.00 \\
Globus pallidus & $65 \pm 7$ & $47 \pm 3$ & $56 \pm 8$ & $65 \pm 2$ & \\
Antero ventral thalamic nucleus & $133 \pm 9$ & $109 \pm 8$ & $120 \pm 9$ & $121 \pm 11$ & \\
Antero hypothalamic nucleus & $74 \pm 9$ & $62 \pm 5$ & $73 \pm 4$ & $75 \pm 8$ & \\
\hline
\end{tabular}




\begin{tabular}{|c|c|c|c|c|c|c|c|}
\hline \multirow[t]{3}{*}{ Brain regions } & \multicolumn{4}{|l|}{$\mathrm{CBF}$} & \multirow{2}{*}{\multicolumn{3}{|c|}{$\begin{array}{l}\text { Two-way ANOVA } \\
P \text { values }\end{array}$}} \\
\hline & \multicolumn{2}{|l|}{ control } & \multicolumn{2}{|l|}{ MDMA } & & & \\
\hline & TRP+ & TRP- & TRP+ & TRP- & Pretreat & ATD & Interaction \\
\hline Tempero parietal cortex & $202 \pm 32$ & $121 \pm 8$ & $102 \pm 13$ & $123 \pm 14$ & 0.01 & & 0.01 \\
\hline Ventral lateral hypothalamic nucleus & $82 \pm 5$ & $70 \pm 5$ & $72 \pm 5$ & $76 \pm 5$ & & & \\
\hline Medial thalamic nucleus & $127 \pm 15$ & $109 \pm 8$ & $97 \pm 4$ & $108 \pm 12$ & & & \\
\hline Ventral lateral thalamic nucleus & $128 \pm 11$ & $100 \pm 6$ & $123 \pm 11$ & $133 \pm 11$ & & & \\
\hline Medial amygdala & $101 \pm 7$ & $80 \pm 6$ & $79 \pm 6$ & $88 \pm 6$ & & & 0.04 \\
\hline Lateral amygdala & $79 \pm 4$ & $66 \pm 3$ & $89 \pm 6$ & $96 \pm 6$ & 0.01 & & \\
\hline Posterior cingulate cortex & $126 \pm 15$ & $100 \pm 8$ & $123 \pm 14$ & $124 \pm 11$ & & & \\
\hline Lateral habenula & $155 \pm 21$ & $121 \pm 10$ & $147 \pm 14$ & $150 \pm 13$ & & & \\
\hline Subthalamic nucleus & $149 \pm 19$ & $117 \pm 7$ & $150 \pm 11$ & $143 \pm 14$ & & & \\
\hline Mamillary body & $141 \pm 17$ & $129 \pm 19$ & $162 \pm 26$ & $153 \pm 36$ & & & \\
\hline Piriform cortex & $89 \pm 8$ & $73 \pm 5$ & $99 \pm 12$ & $93 \pm 13$ & & & \\
\hline Entorhinal cortex & $81 \pm 7$ & $64 \pm 4$ & $95 \pm 5$ & $88 \pm 7$ & 0.00 & 0.04 & \\
\hline Molecular layer & $114 \pm 9$ & $84 \pm 6$ & $113 \pm 9$ & $104 \pm 11$ & & 0.04 & \\
\hline Dorsal subiculum & $95 \pm 7$ & $82 \pm 6$ & $92 \pm 7$ & $88 \pm 5$ & & & \\
\hline Dentate PO & $81 \pm 7$ & $63 \pm 3$ & $78 \pm 6$ & $96 \pm 8$ & 0.02 & & 0.01 \\
\hline Dorsal CA1 & $83 \pm 8$ & $62 \pm 4$ & $81 \pm 5$ & $88 \pm 7$ & 0.06 & & 0.03 \\
\hline $\mathrm{CA} 2$ & $105 \pm 8$ & $77 \pm 6$ & $86 \pm 6$ & $92 \pm 3$ & 0.02 & & \\
\hline Ventral CA1 & $112 \pm 4$ & $93 \pm 9$ & $128 \pm 12$ & $124 \pm 20$ & & & \\
\hline Ventral subiculum & $106 \pm 11$ & $92 \pm 7$ & $108 \pm 7$ & $112 \pm 19$ & & & \\
\hline CA3 & $103 \pm 7$ & $76 \pm 3$ & $99 \pm 4$ & $108 \pm 8^{\#}$ & 0.02 & & 0.01 \\
\hline Ventral tegmental area & $78 \pm 7$ & $58 \pm 3$ & $63 \pm 7$ & $72 \pm 9$ & & & 0.04 \\
\hline Substantia nigra reticulata & $80 \pm 6$ & $70 \pm 4$ & $83 \pm 1$ & $82 \pm 10$ & & & \\
\hline Substantia nigra compacta & $93 \pm 4$ & $80 \pm 4$ & $98 \pm 3$ & $99 \pm 11$ & 0.05 & & \\
\hline Dorsal raphe & $106 \pm 15$ & $95 \pm 12$ & $124 \pm 16$ & $154 \pm 55$ & & & \\
\hline Median raphe & $119 \pm 28$ & $109 \pm 11$ & $141 \pm 16$ & $110 \pm 13$ & & & \\
\hline Paramedian raphe & $96 \pm 23$ & $106 \pm 11$ & $120 \pm 14$ & $103 \pm 9$ & & & \\
\hline Inferior colliculus & $282 \pm 62$ & $229 \pm 12$ & $255 \pm 17$ & $268 \pm 29$ & & & \\
\hline Locus coeruleus & $112 \pm 29$ & $83 \pm 4$ & $134 \pm 9$ & $127 \pm 16$ & 0.02 & & \\
\hline
\end{tabular}

Where effects are significant $(P<0.05)$, levels of significance generated by ANOVA are provided in columns designated Pretreat (main effect of MDMA Pretreatment); ATD (main effect of ATD Treatment); Interaction (Pretreatment x ATD effect).

Using a more rigorous statistical design developed specifically for data from these types of experiment (McCulloch et al., 1982), we confirmed that the ratio of blood flow to metabolism was reset to a significantly lower value in the TRP+ group $(P<0.05)$ despite the more conservative nature of this approach. Repeated measures analysis of variance indicated that this occurred homogeneously in all neuroanatomical regions included in the study. Within each group, significant interregional differences in the ratio of blood flow to metabolism were detected using Greenhouse-Geiser $(P=0.03)$ and HuynhFeldt $(P=0.01)$ corrections, but there was no evidence that this heterogeneity differed between groups (Greenhouse-Geiser, $P=0.64$; Huynh-Feldt, $P=0.71$ ). 


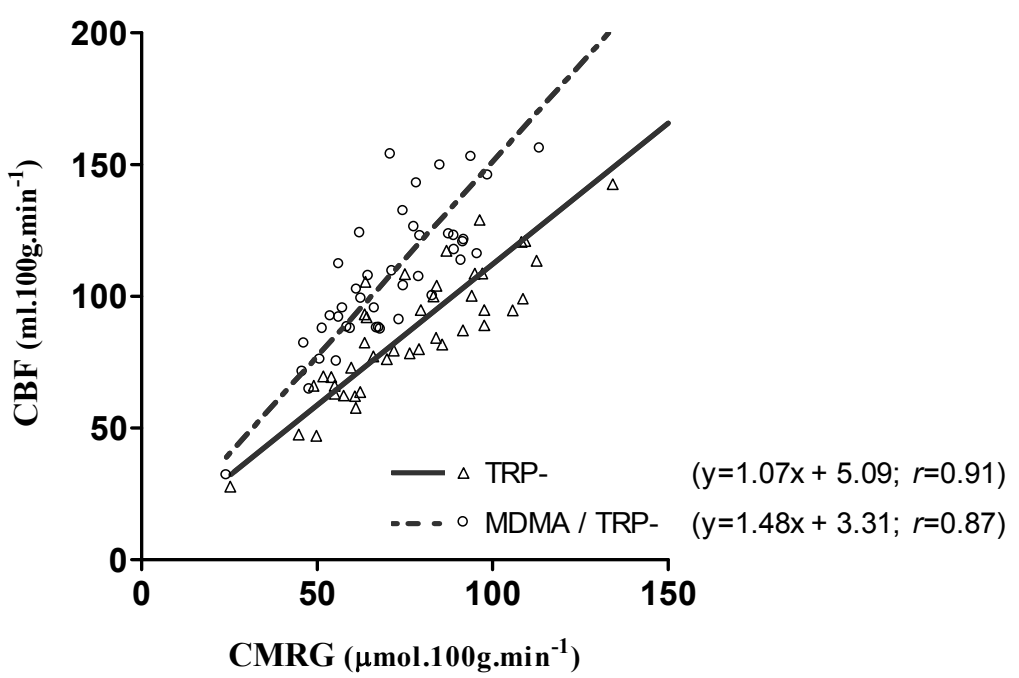

Figure 4 The relationship between local cerebral blood flow (CBF) and local cerebral metabolic rate of glucose (CMRG) in 43 brain regions of interest after ATD treatment in MDMA pretreated rats and controls. Data points represent mean CBF and CMRG for each region together with the best fitting straight line defined by $\mathrm{y}=\mathrm{mx}+\mathrm{c}$, where $\mathrm{x}$ and $\mathrm{y}$ are the variables (CMRG and CBF, respectively), $\mathrm{m}=$ the slope of the line and $\mathrm{c}=$ the intercept.

\section{Discussion}

Although its exact underlying mechanism is not fully understood, MDMAinduced changes in cerebrovascular function have been proposed as a potential contributing factor to the oxidative stress that eventually results in 5-HT neurotoxicity (Darvesh et al., 2002; Ferrington et al., 2006). Therefore, cerebrovascular insufficiency might contribute to an increased incidence of functional impairments, including the development of neuropsychiatric symptoms. As behavioural deficits might only become apparent when the already vulnerable system is additionally challenged, the present study investigated the potential persistent effects of repeated MDMA exposure upon neuronal and cerebrovascular function in rats subjected to an ATD challenge.

The MDMA dosing regime used in the present study is known for its persistent neurodegenerative effect in rats as quantified by measuring reduced densities of 5-HT transporter sites (Battaglia et al., 1987). The present results show a marked decrease in $\left[{ }^{3} \mathrm{H}\right]$-paroxetine-labelled 5-HT reuptake sites throughout the brain 3 weeks after exposure to the drug, thereby confirming its neurotoxic effect. In agreement with other studies using the same dosing regime (Battaglia et al., 1991; Sharkey et al., 1991a), significant and regionally selective decreases in $\left[{ }^{3} \mathrm{H}\right]$-paroxetine binding were observed in both frontal 
cortex areas and subregions of the hippocampus, without affecting the dorsal and medial raphe nuclei. Similar persistent and selective depletions of serotonergic terminals have also been reported after a single exposure to MDMA (Ferrington et al., 2006; Kirilly et al., 2006).

The plasma ratio TRP/ $\Sigma$ LNAA was significantly lowered following ATD in both MDMA pretreated and drug-naïve rats to an equal extent $(-37 \%$ and $-40 \%$ compared to baseline levels, respectively). ATD was accomplished by administrating a TRP-free protein-carbohydrate mixture which has previously been reported to induce profound peripheral TRP decreases (Jans et al., 2008b; Lieben et al., 2004a; Lieben et al., 2004b). However, similar, less robust peripheral effects have also been reported by others and might be due to the strain of rat (Jans et al., 2008a) or sex differences (Jans et al., 2007), as well as pre-existing differences in stress vulnerability (Jans and Blokland, 2008; Olivier et al., 2008). In contrast to previous studies that measured ATD-induced biochemical alterations at the same $4 \mathrm{~h}$ time-point (Jans et al., 2007; Lieben et al., 2004a), no ATD effects were found upon central TRP levels, independent of MDMA pre-treatment. The moderate peripheral TRP depletion might, thus, not have been sufficient to result in a significant decrease of TRP availability to the brain. In addition, it has been suggested that ATD-induced changes in peripheral TRP might not predict central alterations, as distinct metabolic regulation and synthesis mechanisms might be implicated (Olivier et al., 2008; Walther and Bader, 2003). In line with this, no ATD effects upon 5-HT or its metabolite were found and pretreatment with MDMA did not differentially affect this outcome. Although other rat studies of similar experimental design reported decreased 5-HT levels after ATD, specifically in the hippocampus (Jans et al., 2008a; Lieben et al., 2004a; Olivier et al., 2008), it seems unlikely that ATD actually reduces extracellular 5-HT concentrations (Trulson, 1985; van der Plasse et al., 2007) without a prior systemic increase in 5-HT levels (Bel and Artigas, 1996; Fadda et al., 2000a; Stancampiano et al., 1997). Thus, in general, ATD-induced behavioural alterations are not easily ascribed directly to changes in 5-HT neuronal activity and while alternative underlying mechanisms have been suggested (Moore et al., 2000), these remain to be further elucidated. However, rationale for using this method as a challenge tool was that ATDinduced impaired object recognition in rats has been reported consistently (Jans et al., 2008a; Jans et al., 2007; Jans and Blokland, 2008; Lieben et al., 2004b; Olivier et al., 2008), even after only moderate peripheral depletions of the 5-HT precursor (Rutten et al., 2007) and without affecting central 5-HT concentrations (van Donkelaar et al., 2008). Together, these results support the notion that while ATD-induced cognitive dysfunction and affective behavioural alterations in both animals and human subjects can be directly ascribed to a lowering of peripheral TRP concentrations, the underlying mechanism might go beyond a straightforward alteration in the 5-HT system itself.

MDMA is known to decrease the activity of the TPH enzyme (Stone et al., 1987), which catalyzes the hydroxylation of TRP into the naturally occurring 
amino acid 5-hydroxytryptophan (5-HTP). This initial conversion step within the 5-HT biosynthetic pathway is rate-limiting, as TPH is not fully saturated with the amount of TRP normally present in the brain. Therefore, the amount of TRP ultimately converted into 5-HT depends upon substrate availability (Boadle-Biber, 1993) and MDMA-induced decreased TPH activity eventually results in less TRP being converted into the direct 5-HT precursor 5-hydroxytryptophan. The increased hippocampal TRP levels, as observed in the present study, seem to reflect MDMA-induced TPH deactivation and subsequent substrate accumulation. This might additionally provide an explanation for the higher plasma TRP/ $L \mathrm{LNAA}$ ratio found in the TRP+ treatment condition of MDMA pretreated animals compared to drug-naïve rats. It is thus tempting to speculate that the accumulation of central TRP provides the necessary feedback for regulating, in this case diminishing, peripheral TRP uptake into the brain. On the other hand, but along similar lines, an autoregulating compensatory mechanism might increase peripheral TRP levels in the attempt to maintain adequate central metabolism. Thus, although ATD challenge decreases peripheral TRP levels in MDMA pretreated compared to control rats similarly, the increase in central TRP in the MDMA pretreated animals together with the higher peripheral levels might indicate that peripheral TRP metabolism and its uptake into the brain are affected by MDMA-induced 5-HT neurotoxicity.

Furthermore, the significant depletion of 5-HT in both hippocampus and prefrontal cortex of MDMA treated animals is in line with the 5-HT synthesis inhibition due to MDMA-induced TPH deactivation. However, the observed increase in hippocampal 5-HIAA levels seems difficult to explain, as decreased 5-HT synthesis normally parallels less catabolism resulting in less inactive 5-HIAA end product formation. Yet, an increase in 5-HIAA concentration could be a consequence of increased 5-HT catabolism leading to the subsequent depletion of 5-HT concentration. However, decreased 5-HIAA concentration is one of the markers of MDMA-induced disrupted 5-HT metabolism that is normally reported after MDMA abuse (Callahan et al., 2001) and is usually explained by the MDMA-induced inhibition of the intracellular enzyme MAO-A (Leonardi and Azmitia, 1994).

The present study shows a persistent effect of repeated MDMA pretreatment upon CMRG with locally increased, but mainly decreased activity across the anatomically discrete ROIs at different levels of the brain. However, the distribution of MDMA-induced metabolic changes does not exactly mirror the distribution of MDMA-induced 5-HT terminal depletion. This reflects the measure of integrative function afforded by the 2-deoxyglucose technique and therefore, the observed changes in CMRG cannot be directly ascribed to decreased 5-HT functioning in the corresponding areas. These findings are in certainly in keeping with previous studies. A single acute administration of MDMA has been shown to increase metabolic activity in many brain areas (Ando et al., 2006; Balogh et al., 2004; Quate et al., 2004), whereas a persistent 
hypometabolic response and an uncoupling from $\mathrm{CBF}$ has been found in the longer term (Ferrington et al., 2006). In contrast to the effects of MDMA pretreatment upon both CMRG and CBF, ATD did not affect local CMRG or $\mathrm{CBF}$, although some brain regions appeared to be differentially affected by ATD in combination with MDMA pretreatment. Thus, the present results suggest that ATD in animals with serious 5-HT terminal depletion results in a significant upwards resetting of the relationship of CBF to CMRG as compared to the control group.

In the absence of any evidence in this study that an acute decrease in peripheral TRP by means of ATD has any effect upon central 5-HT systems, it is difficult to suggest with any certainty a mechanism by which ATD potentiates the MDMA pretreatment-induced hyperperfusion. As already briefly mentioned above, peripheral 5-HT function may be uncoupled from central 5-HT function as the neurotransmitter is synthesized from TRP by the TPH1 isoform in the periphery and the TPH2 isoform in the brain (Walther and Bader, 2003). In the periphery, one of the main functions of 5-HT is the regulation of vascular tone (Rapport et al., 1948). While it is possible that ATD induces changes in the bioavailability of 5-HT peripherally, and in the blood could remove 5-HT-dependent vasoconstrictor tone, it is unlikely that cerebrovascular function could be influenced in any way so long as the bloodbrain barrier remained intact. A more plausible explanation might be that while the effects of ATD upon central 5-HT systems were below the threshold of detection in brain tissues harvested in this study, they were of sufficient magnitude to reduce cerebrovascular constrictor tone under conditions in which the bioavailability of perivascular 5-HT was compromised by prior MDMA-induced depletion of 5-HT terminals. Moreover the evidence from this and previous studies of a persistent cerebrovascular effect of MDMA might suggest that the cerebrovasculature is more sensitive to the loss of endogenous 5-HT than is the neuropil.

To summarize, the present results show that MDMA pretreatment and ATD interact to produce a small but significant potentiation of MDMAinduced cerebrovascular dysfunction. The resulting decrease in cerebrovascular tone and consequent hyperperfusion could indicate a predisposition to cerebrovascular accident and haemorrhagic stroke in particular. More widely, it is of interest that the 5-HT dysfunction induced by MDMA is paralleled, albeit more subtly, by the decrease in transporter function found in some cases of depression, for which ATD is used as a clinical diagnostic probe. While it would stretch the available evidence to suggest that cerebrovascular dysfunction is the root cause of depressive illness, it is tempting to speculate that changes in $\mathrm{CBF}$ might contribute to the behavioural dysfunction of depression.

\section{Acknowledgements}

This work was funded by a EUROGENDIS award to E.L.v.D. 


\section{References}

Ando RD, Benko A, Ferrington L, Kirilly E, Kelly PA, Bagdy G (2006). Partial lesion of the serotonergic system by a single dose of MDMA results in behavioural disinhibition and enhances acute MDMA-induced social behaviour on the social interaction test. Neuropharmacology 50(7): 884-896.

Balogh B, Molnar E, Jakus R, Quate L, Olverman HJ, Kelly PA, Kantor S, Bagdy G (2004). Effects of a single dose of 3,4-methylenedioxymethamphetamine on circadian patterns, motor activity and sleep in drug-naive rats and rats previously exposed to MDMA. Psychopharmacology (Berl) 173(3-4): 296-309.

Battaglia G, Yeh SY, O'Hearn E, Molliver ME, Kuhar MJ, De Souza EB (1987). 3,4Methylenedioxymethamphetamine and 3,4-methylenedioxyamphetamine destroy serotonin terminals in rat brain: quantification of neurodegeneration by measurement of [3H]paroxetine-labeled serotonin uptake sites. J Pharmacol Exp Ther 242(3): 911-916.

Battaglia G, Yeh SY, De Souza EB (1988). MDMA-induced neurotoxicity: parameters of degeneration and recovery of brain serotonin neurons. Pharmacol Biochem Behav 29(2): 269-274.

Battaglia G, Sharkey J, Kuhar MJ, de Souza EB (1991). Neuroanatomic specificity and time course of alterations in rat brain serotonergic pathways induced by MDMA (3,4methylenedioxymethamphetamine): assessment using quantitative autoradiography. Synapse 8(4): 249-260.

Baumann MH, Wang X, Rothman RB (2007). 3,4-Methylenedioxymethamphetamine (MDMA) neurotoxicity in rats: a reappraisal of past and present findings. Psychopharmacology (Berl) 189(4): 407-424.

Bel N, Artigas F (1996). Reduction of serotonergic function in rat brain by tryptophan depletion: effects in control and fluvoxamine-treated rats. J Neurochem 67(2): 669-676.

Bell CJ, Hood SD, Nutt DJ (2005). Acute tryptophan depletion. Part II: clinical effects and implications. Aust N Z J Psychiatry 39(7): 565-574.

Boadle-Biber MC (1993). Regulation of serotonin synthesis. Prog Biophys Mol Biol 60(1): 1-15.

Booij L, Van der Does W, Benkelfat C, Bremner JD, Cowen PJ, Fava M, Gillin C, Leyton M, Moore P, Smith KA, Van der Kloot WA (2002). Predictors of mood response to acute tryptophan depletion. A reanalysis. Neuropsychopharmacology 27(5): 852-861.

Booij L, van der Does AJ, Haffmans PM, Spinhoven P, McNally RJ (2005). Acute tryptophan depletion as a model of depressive relapse: behavioural specificity and ethical considerations. Br J Psychiatry 187: 148-154.

Cahir M, Ardis TC, Elliott JJ, Kelly CB, Reynolds GP, Cooper SJ (2007). Acute tryptophan depletion does not alter central or plasma brain-derived neurotrophic factor in the rat. Eur Neuropsychopharmacol: 317-322.

Callahan BT, Cord BJ, Ricaurte GA (2001). Long-term impairment of anterograde axonal transport along fiber projections originating in the rostral raphe nuclei after treatment with fenfluramine or methylenedioxymethamphetamine. Synapse 40(2): 113-121.

Commins DL, Vosmer G, Virus RM, Woolverton WL, Schuster CR, Seiden LS (1987). Biochemical and histological evidence that methylenedioxymethylamphetamine (MDMA) is toxic to neurons in the rat brain. J Pharmacol Exp Ther 241(1): 338-345.

Curran HV, Verheyden SL (2003). Altered response to tryptophan supplementation after longterm abstention from MDMA (ecstasy) is highly correlated with human memory function. Psychopharmacology (Berl) 169(1): 91-103.

Darvesh AS, Shankaran M, Gudelsky GA (2002). 3,4-Methylenedioxymethamphetamine produces glycogenolysis and increases the extracellular concentration of glucose in the rat brain. J Pharmacol Exp Ther 301(1): 138-144.

De Souza EB, Kuyatt BL (1987a). Autoradiographic localization of 3H-paroxetine-labeled serotonin uptake sites in rat brain. Synapse 1(5): 488-496. 
De Souza EB, Kuyatt BL (1987b). Autoradiographic localization of 3H-paroxetine-labeled serotonin uptake sites in rat brain. Synapse 1(5): 488-496.

Dixon WJ (1959). Analysis of extreme values. Ann Math Stat 21: 488-506.

Domek-Lopacinska K, Strosznajder JB (2008). The effect of selective inhibition of cyclic GMP hydrolyzing phosphodiesterases 2 and 5 on learning and memory processes and nitric oxide synthase activity in brain during aging. Brain Res 1216: 68-77.

Fadda F (2000). Tryptophan-Free Diets: A Physiological Tool to Study Brain Serotonin Function. News Physiol Sci 15: 260-264.

Fadda F, Cocco S, Rossetti ZL, Melis G, Stancampiano R (2000a). A tryptophan-free diet markedly reduces frontocortical 5-HT release, but fails to modify ethanol preference in alcohol-preferring (sP) and non-preferring (sNP) rats. Behav Brain Res 108(2): 127-132.

Fadda F, Cocco S, Stancampiano R (2000b). A physiological method to selectively decrease brain serotonin release. Brain Res Brain Res Protoc 5(3): 219-222.

Fernstrom JD, Wurtman RJ (1972). Brain serotonin content: physiological regulation by plasma neutral amino acids. Science 178(59): 414-416.

Fernstrom JD (1979). Diet-induced changes in plasma amino acid pattern: effects on the brain uptake of large neutral amino acids, and on brain serotonin synthesis. J Neural Transm Suppl(15): 55-67.

Fernstrom JD (1983). Role of precursor availability in control of monoamine biosynthesis in brain. Physiol Rev 63(2): 484-546.

Fernstrom JD (1986). Acute and chronic effects of protein and carbohydrate ingestion on brain tryptophan levels and serotonin synthesis. Nutr Rev 44 Suppl: 25-36.

Ferrington L, Kirilly E, McBean DE, Olverman HJ, Bagdy G, Kelly PA (2006). Persistent cerebrovascular effects of MDMA and acute responses to the drug. Eur J Neurosci 24(2): 509-519.

Ford I, McColl JH, McCormack AG, McCrory SJ (1991). Statistical issues in the analysis of neuroimages. J Cereb Blood Flow Metab 11(2): A89-95.

Geiser S, Greenhouse SW (1958). Box's results on the use of the F distribution in multivariate analysis. Ann Math Statist 29(885): 885--891.

Green AR, Mechan AO, Elliott JM, O'Shea E, Colado MI (2003). The pharmacology and clinical pharmacology of 3,4-methylenedioxymethamphetamine (MDMA, "ecstasy"). Pharmacol Rev 55(3): 463-508.

Guillot C (2007). Is recreational ecstasy (MDMA) use associated with higher levels of depressive symptoms? J Psychoactive Drugs 39(1): 31-39.

Hood SD, Bell CJ, Nutt DJ (2005). Acute tryptophan depletion. Part I: rationale and methodology. The Australian and New Zealand journal of psychiatry 39(7): 558-564.

Jans L, Korte-Bouws G, Korte S, Blokland A (2008a). The effects of acute tryptophan depletion on affective behaviour and cognition in Brown Norway and Sprague Dawley rats. J Psychopharmacol.

Jans LA, Lieben CK, Blokland A (2007). Influence of sex and estrous cycle on the effects of acute tryptophan depletion induced by a gelatin-based mixture in adult Wistar rats. Neuroscience 147(2): 304-317.

Jans LA, Blokland A (2008). Influence of chronic mild stress on the behavioural effects of acute tryptophan depletion induced by a gelatin-based mixture. Behav Pharmacol 19(7): 706-715.

Jans LA, Lieben CK, Smits LT, Blokland A (2008b). Pharmacokinetics of acute tryptophan depletion using a gelatin-based protein in male and female Wistar rats. Amino Acids.

Jennrich R, Sampson P, Frane J. 1990. Analysis of variance and covariance with repeated measures. In: BMDP Statistical Software Manual, Vol. 1 (Dixon, W.J., Brown, M.B., Engelman, L., Jenrich R.I.). Berkeley, University of California Press, pp 486-527.

Kelly PA, Ritchie IM, Arbuthnott GW (1995). Inhibition of neuronal nitric oxide synthase by 7 nitroindazole: effects upon local cerebral blood flow and glucose use in the rat. J Cereb Blood Flow Metab 15(5): 766-773. 
Kirilly E, Benko A, Ferrington L, Ando RD, Kelly PA, Bagdy G (2006). Acute and long-term effects of a single dose of MDMA on aggression in Dark Agouti rats. Int J Neuropsychopharmacol 9(1): 63-76.

Leonardi ET, Azmitia EC (1994). MDMA (ecstasy) inhibition of MAO type A and type B: comparisons with fenfluramine and fluoxetine (Prozac). Neuropsychopharmacology 10(4): 231-238.

Lieben CK, Blokland A, Westerink B, Deutz NE (2004a). Acute tryptophan and serotonin depletion using an optimized tryptophan-free protein-carbohydrate mixture in the adult rat. Neurochem Int 44(1): 9-16.

Lieben CK, van Oorsouw K, Deutz NE, Blokland A (2004b). Acute tryptophan depletion induced by a gelatin-based mixture impairs object memory but not affective behavior and spatial learning in the rat. Behav Brain Res 151(1-2): 53-64.

Markus CR, Panhuysen G, Tuiten A, Koppeschaar H, Fekkes D, Peters ML (1998). Does carbohydrate-rich, protein-poor food prevent a deterioration of mood and cognitive performance of stress-prone subjects when subjected to a stressful task? Appetite 31(1): 4965.

McCulloch J, Kelly PA, Ford I (1982). Effect of apomorphine on the relationship between local cerebral glucose utilization and local cerebral blood flow (with an appendix on its statistical analysis). J Cereb Blood Flow Metab 2(4): 487-499.

Moore P, Landolt HP, Seifritz E, Clark C, Bhatti T, Kelsoe J, Rapaport M, Gillin JC (2000). Clinical and physiological consequences of rapid tryptophan depletion. Neuropsychopharmacology 23(6): 601-622.

Moreno FA, Heninger GR, McGahuey CA, Delgado PL (2000). Tryptophan depletion and risk of depression relapse: a prospective study of tryptophan depletion as a potential predictor of depressive episodes. Biol Psychiatry 48(4): 327-329.

Neumeister A (2003). Tryptophan depletion, serotonin, and depression: where do we stand? Psychopharmacol Bull 37(4): 99-115.

Nichols DE, Oberlender R (1990). Structure-activity relationships of MDMA and related compounds: a new class of psychoactive drugs? Ann N Y Acad Sci 600: 613-623; discussion 623-625.

O'Hearn E, Battaglia G, De Souza EB, Kuhar MJ, Molliver ME (1988). Methylenedioxyamphetamine (MDA) and methylenedioxymethamphetamine (MDMA) cause selective ablation of serotonergic axon terminals in forebrain: immunocytochemical evidence for neurotoxicity. J Neurosci 8(8): 2788-2803.

Olivier JD, Jans LA, Korte-Bouws GA, Korte SM, Deen PM, Cools AR, Ellenbroek BA, Blokland A (2008). Acute tryptophan depletion dose dependently impairs object memory in serotonin transporter knockout rats. Psychopharmacology (Berl) 200(2): 243-254.

Paxinos G, Watson C (1998). The Rat Brain in Stereotaxic Coordinates, 4th ed., vol. 4. Elsevier Academic Press, San Diego. (4th ed).

Quate L, McBean DE, Ritchie IM, Olverman HJ, Kelly PA (2004). Acute methylenedioxymethamphetamine administration: effects on local cerebral blood flow and glucose utilisation in the Dark Agouti rat. Psychopharmacology (Berl) 173(3-4): 287-925.

Rapport MM, Green AA, Page IH (1948). Serum vasoconstrictor, serotonin; isolation and characterization. J Biol Chem 176(3): 1243-1251.

Ricaurte GA, Yuan J, McCann UD (2000). (+/-)3,4-Methylenedioxymethamphetamine ('Ecstasy')-induced serotonin neurotoxicity: studies in animals. Neuropsychobiology 42(1): 5 10.

Rutten K, Lieben C, Smits L, Blokland A (2007). The PDE4 inhibitor rolipram reverses object memory impairment induced by acute tryptophan depletion in the rat. Psychopharmacology (Berl) 192(2): 275-282.

Sakurada O, Kennedy C, Jehle J, Brown JD, Carbin GL, Sokoloff L (1978). Measurement of local cerebral blood flow with iodo [14C] antipyrine. Am J Physiol 234(1): H59-66.

Schmidt CJ (1987). Neurotoxicity of the psychedelic amphetamine, methylenedioxymethamphetamine. J Pharmacol Exp Ther 240(1): 1-7. 
Sharkey J, McBean DE, Kelly PA (1991a). Alterations in hippocampal function following repeated exposure to the amphetamine derivative methylenedioxymethamphetamine ("Ecstasy"). Psychopharmacology (Berl) 105(1): 113-118.

Sharkey J, McBean DE, Kelly PA (1991b). Alterations in hippocampal function following repeated exposure to the amphetamine derivative methylenedioxymethamphetamine ("Ecstasy"). Psychopharmacology (Berl) 105(1): 113-118.

Sokoloff L, Reivich M, Kennedy C, Des Rosiers MH, Patlak CS, Pettigrew KD, Sakurada O, Shinohara M (1977). The [14C]deoxyglucose method for the measurement of local cerebral glucose utilization: theory, procedure, and normal values in the conscious and anesthetized albino rat. J Neurochem 28(5): 897-916.

Sokoloff L (1981). The deoxyglucose method for the measurement of local glucose utilization and the mapping of local functional activity in the central nervous system. Int Rev Neurobiol 22: 287-333.

Stancampiano R, Melis F, Sarais L, Cocco S, Cugusi C, Fadda F (1997). Acute administration of a tryptophan-free amino acid mixture decreases 5 -HT release in rat hippocampus in vivo. Am J Physiol 272(3 Pt 2): R991-994.

Stone DM, Merchant KM, Hanson GR, Gibb JW (1987). Immediate and long-term effects of 3,4-methylenedioxymethamphetamine on serotonin pathways in brain of rat. Neuropharmacology 26(12): 1677-1683.

Taffe MA, Davis SA, Yuan J, Schroeder R, Hatzidimitriou G, Parsons LH, Ricaurte GA, Gold LH (2002). Cognitive performance of MDMA-treated rhesus monkeys: sensitivity to serotonergic challenge. Neuropsychopharmacology 27(6): 993-1005.

Taffe MA, Huitron-Resendiz S, Schroeder R, Parsons LH, Henriksen SJ, Gold LH (2003). MDMA exposure alters cognitive and electrophysiological sensitivity to rapid tryptophan depletion in rhesus monkeys. Pharmacology, biochemistry, and behavior 76(1): 141-152.

Trulson ME (1985). Dietary tryptophan does not alter the function of brain serotonin neurons. Life Sci 37(11): 1067-1072.

Van der Does AJ (2001). The mood-lowering effect of tryptophan depletion: possible explanation for discrepant findings. Arch Gen Psychiatry 58(2): 200-202.

van der Plasse G, Meerkerk DT, Lieben CK, Blokland A, Feenstra MG (2007). Lack of evidence for reduced prefrontal cortical serotonin and dopamine efflux after acute tryptophan depletion. Psychopharmacology (Berl) 195(3): 377-385.

van Donkelaar EL, Rutten K, Blokland A, Akkerman S, Steinbusch HW, Prickaerts J (2008). Phosphodiesterase 2 and 5 inhibition attenuates the object memory deficit induced by acute tryptophan depletion. Eur J Pharmacol 600(1-3): 98-104.

van Eijk HM, Rooyakkers DR, Deutz NE (1993). Rapid routine determination of amino acids in plasma by high-performance liquid chromatography with a 2-3 microns Spherisorb ODS II column. J Chromatogr 620(1): 143-148.

Walther DJ, Bader M (2003). A unique central tryptophan hydroxylase isoform. Biochem Pharmacol 66(9): 1673-1680. 


\section{Chapter}

\section{Stress-mediated decreases in brain- derived neurotrophic factor as potential confounding factor for acute tryptophan depletion-induced neurochemical effects}

Eva L. van Donkelaar ${ }^{1}$, Daniël L.A. van den Hove ${ }^{1}$, Arjan Blokland ${ }^{2}$, Harry W.M. Steinbusch ${ }^{1}$, Jos Prickaerts ${ }^{1}$

${ }^{1}$ Department of Neuroscience, Faculty of Health, Medicine and Life Sciences, School for Mental Health and Neuroscience, Maastricht University, The Netherlands

${ }^{2}$ Department of Neuropsychology and Psychopharmacology, Faculty of Psychology and Neuroscience, Maastricht University, The Netherlands 



\begin{abstract}
Acute tryptophan depletion (ATD) is extensively used to investigate the implication of serotonin (5-hydroxytryptamine; 5-HT) in the onset and treatment of depression and cognitive disorders. Brain-derived neurotrophic factor (BDNF) is strongly linked to the 5-HT system and plays an essential role in mood and memory processes. The present study investigated the effects of ATD upon BDNF in serum, hippocampus and prefrontal cortex in the rat to further explore the underlying mechanism of ATD. ATD significantly decreased peripheral tryptophan (TRP) levels and moderately interrupted 5-HT metabolism $4 \mathrm{~h}$ after administration of the nutritional mixture. Although no direct effects of ATD upon serum or brain BDNF concentrations were found, a stress-mediated, decrease in BDNF was observed in the prefrontal cortex. Moreover, brain TRP levels correlated positively with BDNF in both the prefrontal cortex and hippocampus. Thus, BDNF-mediated mechanisms due to ATD and/or its application stress might underlie ATD-induced neurochemical and behavioural alterations.
\end{abstract}




\section{Introduction}

The implication of the serotonin (5-hydroxytryptamine; 5-HT) system and serotonergic vulnerability factors in the onset and treatment of depressive symptomatology is being extensively investigated with so-called serotonergic challenge tools. Most widely used, both clinically and pre-clinically, is the acute tryptophan depletion (ATD) method, which manipulates the availability of the essential amino acid tryptophan (TRP), the dietary 5-HT precursor (Bell et al., 2005; Hood et al., 2005; Neumeister, 2003; Young, 1993). Through administration of a TRP-free nutritional mixture, plasma TRP levels are decreased and thus less of the precursor is available to be converted into 5-HT (Fadda et al., 2000b; Fernstrom, 1983). Since this method is relatively easy to apply and non-toxic, it can be safely and repeatedly used in both human subjects and rodents (Blokland et al., 2004; Fadda et al., 2000b).

ATD is known to substantially lower peripheral TRP levels and hippocampal 5-HT concentrations in rats (Lieben et al., 2004a) and profound disrupted 5-HT metabolism has been reported in human subjects (Carpenter et al., 1998; Williams et al., 1999). However, actual decreases in extracellular 5-HT have only been observed after ATD in combination with blockade of 5-HT reuptake (Bel and Artigas, 1996; Fadda et al., 2000a; Stancampiano et al., 1997). Nevertheless, ATD consistently induces object memory deficits in rats (Jans et al., 2007a; Lieben et al., 2004b; Lieben et al., 2005; Olivier et al., 2008; Rutten et al., 2007; van Donkelaar et al., 2008). In addition, impaired memory processes have been reported in both healthy human subjects and subjects with preexisting abnormal central 5-HT regulation, whereas mood-lowering effects are only observed in the latter (Booij et al., 2002; Booij et al., 2003; Klaassen et al., 1999; Neumeister et al., 2002; Neumeister et al., 2006; e.g. Smith et al., 1997). It thus remains unclear to what extent ATD-induced cognitive dysfunction and other behavioural changes can be directly ascribed to alterations in 5-HT neuronal activity (Moore et al., 2000; van der Plasse et al., 2007).

Extensively studied is the link between 5-HT and brain-derived neurotrophic factor (BDNF), a member of the nerve growth factor gene family and the most abundant of the neurotrophins in the brain (Ernfors et al., 1990). BDNF plays an important role in differentiation, outgrowth and survival of peripheral and central neurons during brain development (Ghosh et al., 1994; McAllister et al., 1995) and in neuronal recovery after brain damage (Hicks et al., 1999). Moreover, the protein appears to have important neurotransmittermodulating effects throughout adult life upon monoamine (Mattson et al., 2004; Siuciak et al., 1996) and neuropeptide function (Croll et al., 1994; Nawa et al., 1994). High levels of BDNF mRNA are found in the hippocampus and the cerebral cortex (Conner et al., 1997; Ernfors et al., 1990; Hofer et al., 1990), which explains the involvement of BDNF in mechanisms implicated in depression, including cognitive dysfunction. 
5-HT stimulates the expression of BDNF through production of cyclic adenosine monophosphate (cAMP) and activation of cAMP-response elementbinding (CREB) protein (Duman et al., 1997; Duman, 1998; Mattson et al., 2004). On the other hand, BDNF promotes the function, growth and sprouting of 5-HT neurons (Mamounas et al., 1995; Mamounas et al., 2000) and increases 5-HT synthesis by enhancing tryptophan hydroxylase (TPH) mRNA levels (Siuciak et al., 1998). It has been shown that BDNF mediates the effects of antidepressants and has itself antidepressant properties (Altar et al., 2003; Nibuya et al., 1995; Nibuya et al., 1996). Furthermore, decreased levels of BDNF in serum have been reported in depressed patients, whereas antidepressant treatment is able to reverse the attenuated BDNF levels (Gonul et al., 2005; Karege et al., 2005; Shimizu et al., 2003). While a decrease in circulating BDNF was found in patients with remitted major depressive disorder after ATD, a suggested compensatory increase was found in healthy subjects (Neumeister et al., 2005). In rats, however, no ATD-induced changes were observed upon peripheral or central BDNF levels (Cahir et al., 2008). Of note, BDNF is extremely stress-responsive (Smith et al., 1995a), which is supported by studies showing that both acute and repeated immobilization stress considerably decreases BDNF mRNA expression in the rat hippocampus, even within $1 \mathrm{~h}$ (Smith et al., 1995b; Ueyama et al., 1997). Stressinduced changes in BDNF are not only glucocorticoid-mediated (Prickaerts et al., 2006), but might be regulated additionally by alterations in the activity of specific 5-HT receptors (Vaidya et al., 1997; Vollmayr et al., 2000). Experimental stressors related to ATD application procedures might, thus, also influence central BDNF levels.

Neurotrophins in general play an important role in neuronal plasticity, including long-term potentiation (LTP). BDNF has been shown to directly modulate hippocampal LTP (Figurov et al., 1996) and mice lacking BDNF show impaired LTP regulation (Korte et al., 1995). As LTP is assumed to be the underlying substrate of learning and memory processes (Bliss and Collingridge, 1993), BDNF is thought to be a potential mediator of memory formation in general and is known to be required specifically for memory consolidation (Lee et al., 2004). ATD in human subjects selectively impairs memory consolidation (Riedel et al., 1999), which has been attributed to lowered 5-HT in hippocampal areas (Riedel, 2004), however, a direct link to changes in BDNF remains to be elucidated. It is nevertheless tempting to speculate that the consistently reported ATD-induced cognitive dysfunctions, as observed in both healthy and vulnerably subjects, but also in rats, might be attributed, directly or indirectly, to BDNF-mediated alterations in learning and memory processing.

The aim of the present study was to further explore the underlying mechanism of ATD in rats by determining its effects upon BDNF levels in serum, hippocampus and prefrontal cortex while controlling for possible confounding stress effects of the specific experimental procedures. In the first 
place, it was hypothesized that reduced 5-HT metabolism, due to depletion of its precursor, would result in alterations of peripheral and central BDNF levels. In addition, we speculated upon the potential of experimental stressors to decrease basal BDNF levels. Possible stress-induced decreased central BDNF concentrations might alter TRP metabolism and synthesis into 5-HT, thereby interfering with pure ATD effects. These observations could contribute to a better understanding of ATD-induced cognitive dysfunction and affective behavioural changes.

\section{Experimental procedures}

\section{Animals}

Forty six-month-old male Wistar rats (Charles River, The Netherlands) were used, weighing between 450-500 $\mathrm{g}$ at the start of the experiments. On arrival, animals were housed individually in standard Makrolon cages on sawdust bedding and maintained on a reversed 12:12-h light-dark schedule (lights on from 18:00 $\mathrm{h}-06: 00 \mathrm{~h})$ in a temperature-controlled $\left(21 \pm 2^{\circ} \mathrm{C}\right)$ and airconditioned colony room. Background noise was provided by a radio playing softly at all times. Handling and treatment of the animals was not started until two weeks after arrival and food and tap water were freely available throughout this period.

All efforts were made to minimize the number of animals used and their suffering. Experimental procedures were approved by the local ethical Committee on Animal Experimentation of the Maastricht University and were in accordance with governmental guidelines.

\section{Nutritional mixture}

Acute reduction of plasma TRP levels was accomplished by administering a TRP-free protein-carbohydrate nutritional mixture (Gelatine hydrolysate, Solugel ${ }^{\circledR}$; PB Gelatins, Tessenderlo, Belgium). Compared to pure amino acid mixtures, the gelatin-based mixture comprises the entire range of amino acids in the form of peptides, as found in normal food and naturally lacks the essential amino acid TRP (for amino acid content and composition of the mixture see: van Donkelaar et al., 2008). The addition of carbohydrates (Glucodry 210; Amylumgroup, Koog aan de Zaan, The Netherlands) activates the insulin response and the resulting increase in protein synthesis promotes an optimized reduction in plasma TRP levels (Fernstrom, 1986; Lieben et al., 2004a; Markus et al., 1998).

To the control mixture, L-tryptophan (Sigma, Zwijndrecht, The Netherlands) was added in an amount equivalent to $0.28 \%$ of total protein content, as adapted from Lieben et al. (2004a). Potassium chloride (KCl) and 
calcium chloride dihydrate $\left(\mathrm{CaCl}_{2} \cdot 2 \mathrm{H}_{2} \mathrm{O}\right)$ were obtained from Merck (Darmstadt, Germany). Nutritional mixtures were dissolved in distilled water and freshly prepared in the afternoon the day before the actual experiment and stored at $4{ }^{\circ} \mathrm{C}$ until the day of the experiment.

\section{ATD treatment}

After adaptation to the new environment and the reversed light-dark cycle, animals were handled and habituated to the oral administrations by gavage, two weeks before the start of the experiments. Rats were deprived of food overnight $( \pm 15 \mathrm{~h}$ ) prior to the experimental day to avoid TRP uptake from the standard animal chow. Water remained freely available at all times.

Experimental procedures took place in the dark phase of the animals' lightdark cycle, between 9:00 and 14:00 h. Animals were randomly assigned to one of three treatment conditions ( $n=10$ per group), receiving either saline or the nutritional mixture with (TRP+ group) or without TRP (TRP- group). Each experimental dose contained $4 \mathrm{~g}$ of Solugel ${ }^{\circledR}$ and $2 \mathrm{~g}$ of Glucodry 210 per $\mathrm{kg}$ bodyweight and was administered twice in a volume of $10 \mathrm{ml} / \mathrm{kg}$ with a $90 \mathrm{~min}$ interval, $240 \mathrm{~min}$ prior to decapitation.

In addition, a fourth experimental group of animals $(n=10)$ was included. These animals did not receive any oral treatments and were not deprived of food the night before the experimental day. This group served to measure baseline central BDNF levels and was used as a control group against the saline treated group to discard potential stress effects of the food deprivation in general and the oral applications by gavage in particular.

\section{Collection and preparation of blood samples}

For determination of plasma amino acid concentrations and BDNF in serum, baseline blood samples were taken by means of saphenous vein puncture (Hem et al., 1998), 30 min prior to the first treatment administration. Approximately $100 \mu \mathrm{l}$ (for $\pm 50 \mu \mathrm{l}$ plasma) was captured into heparin coated tubes (Microvette $^{\circledR}$ CB 300, Sarstedt, Germany) which were kept on ice until centrifuged. Another $50 \mu \mathrm{l}$ (for $\pm 25 \mu \mathrm{l}$ serum) was captured in standard cups and left at room temperature for approximately $1 \mathrm{~h}$ followed by $1 \mathrm{~h}$ at $4{ }^{\circ} \mathrm{C}$. A second blood sample was taken by collecting trunk blood at time of decapitation, i.e. $4 \mathrm{~h}$ after the first treatment administration. All samples were centrifuged at $4{ }^{\circ} \mathrm{C}$ using a Hettish EBA 12 centrifuge with $10 \mathrm{~min}$ at 14,000 x g for separating the plasma and $15 \mathrm{~min}$ at $2000 \mathrm{x} \mathrm{g}$ for separating serum. Both plasma and serum were transferred into cups which were immediately frozen in liquid nitrogen and stored at $-80^{\circ} \mathrm{C}$ until further analysis. 


\section{Collection and preparation of tissue samples}

At time of decapitation, prefrontal cortex and hippocampus were dissected out bilaterally. Right hemisphere tissue was homogenized for determination of central amino acid and 5-HT, the 5-HT metabolite (5-hydroxyindoleacetic acid; 5-HIAA) and dopamine (DA) concentrations, whereas left hemisphere tissue was used for central BDNF analysis. After dissection, tissue was weighted on a microbalance in pre-weighed $2.0 \mu \mathrm{l}$ screw-lid cups and immediately frozen in liquid nitrogen prior to storage at $-80^{\circ} \mathrm{C}$. All tissue dissections were performed by the same investigator (E. L. van Donkelaar) to preserve consistency. At a later stage, $250 \mu \mathrm{l}$ of a 5 -sulfosalicylic acid solution, including $15 \mu \mathrm{l}$ (of $2 \mathrm{mM}$ per $85 \mu \mathrm{l}$ supernatant) of ascorbic acid to protect against monoamine degradation processes by oxidation, was added to the tissue. Further, the cups were filled with approximately $0.3 \mathrm{~g}$ of glass beads $(1.0 \mathrm{~mm}$ diameter $)$ and brain tissue was homogenized in a mini-bead beater set at high speed $(3 \times 30 \mathrm{~s}$ with cooling in between runs). Subsequently, samples were stored at $-80{ }^{\circ} \mathrm{C}$ until further analyses.

\section{Biochemical analysis}

Plasma and brain amino acid concentrations were quantified using fully automated high-performance liquid chromatography (HPLC) with fluorescence detection (van Eijk et al., 1993). The concentrations of free plasma and central amino acids were expressed as $\mu \mathrm{mol} / \mathrm{l}$, brain concentrations as $\mathrm{ng} / \mathrm{mg}$. Since TRP competes at the blood-brain barrier with the other large neutral amino acids (LNAAs; leucine [LEU], isoleucine [ILE], phenylalaline [PHE], tyrosine [TYR], valine [VAL]) for the same amino acid L-carrier transport system (Fernstrom and Wurtman, 1972; Fernstrom, 1983), a decrease of the ratio of TRP to the sum of the other LNAAs in plasma (TRP/ LLNAA) is normally used as the best predictor of reduced central availability of TRP (Fernstrom, 1979, 1983). Central concentrations of 5-HT, 5-HIAA and DA in the hippocampus and prefrontal cortex were quantified by automated HPLC with dual electrochemical detection.

BDNF in serum and brain homogenates was measured as previously described (Scheepens et al., 2003; Van den Hove et al., 2006) and according to manufacturer's instructions using the BDNF $\mathrm{E}_{\max }{ }^{\circledR}$ ImmunoAssay system from Promega (Madison, MI, USA) and high affinity EIA/RIA 96-well plates (Corning, NY, USA). The resultant absorbency was read using a microplate reader at $405 \mathrm{~nm}$ (Biorad, CA, USA). Serum and tissue homogenate samples were diluted within the range of the standard curve and expressed as $\mathrm{pg} / \mathrm{l}$ and $\mathrm{ng} / \mathrm{g}$. All samples were measured in duplicate. 


\section{Statistical analyses}

Unless otherwise stated, all data were evaluated statistically using independent Student's $t$-tests. To reveal procedural stress effects, the group that received no treatment at all was compared with the saline condition. Saline and TRP+ treatment were compared to establish differences between the two control groups, while ATD effects were revealed by comparing the TRP- group with the TRP + control group. Because multiple (three) comparisons were made, the level of significance was adjusted (post hoc correction) to $P<0.05 / 3=P<0.017$.

Mean concentrations of plasma amino acids were determined for each treatment condition and time-point separately and TRP/ $\Sigma$ LNAA ratios were calculated accordingly. Changes in the ratio TRP/ $\Sigma$ LNAA and serum BDNF over time were analyzed by means of two-way analysis of variance (ANOVA) with Treatment as between-subject factor and Time as within-subject variable. Subsequently, separate one-way ANOVAs were performed to analyze differences between treatment conditions per time-point. Post hoc multiple (three) comparisons were made, as mentioned above, to further characterize the treatment effects (significance set at $P<0.017$ ). Mean central amino acid concentrations, 5-HT, 5-HIAA, 5-HT turnover rate (5-HIAA/5-HT) and DA levels, as well as central BDNF levels were analyzed for hippocampus and prefrontal cortex separately. The relationship between the most prominent peripheral and central parameters was examined by calculating Pearson's correlation coefficient $\left(r_{\mathrm{p}}\right)$ with the levels of significance set at $P<0.05$.

Extreme values representing artefacts in the raw data arising from the HPLC analyses and BDNF ELISA were analyzed by means of Dixon's Extremity test (Dixon, 1959) and only those values for a given animal were excluded from statistical analysis. Data are represented as mean \pm standard error of the mean (S.E.M.).

\section{Results}

\section{Plasma amino acids}

An overview of the concentration of the LNAAs in plasma can be found in Table 1. No treatment effects were found at baseline and only the TRP levels in plasma were selectively decreased $4 \mathrm{~h}$ after ATD compared to the TRP+ control treatment $[t(17)=5.964 ; P<0.017$. Decreased levels of TYR $[t(18)=$ 6.317; $P<0.017]$, ILE $[t(18)=7.156 ; P<0.017]$ and LEU $[t(18)=5.736$; $P<0.017]$ were found after TRP+ treatment compared to saline treatment $4 \mathrm{~h}$ after treatment initiation.

ATD decreased the ratio TRP/ $/$ LNAA in plasma (Fig. 1) over time $[F(1$, 34) $=22.79 ; P<0.001]$, however, not in the same way for all treatment conditions $[F(3,34)=3.66 ; P<0.05]$. An overall treatment effect was found 
$[F(3,34)=3.94 ; P<0.05]$ and post-hoc analysis showed that the TRP+ treatment significantly differed from the TRP-group and from the group that did not receive any treatment.

Table 1 Large neutral amino acid (LNAA) concentrations (mean \pm S.E.M. in $\mu$ mol/l) in rat plasma at baseline and $240 \mathrm{~min}$ after the first oral administration with either saline or the proteincarbohydrate nutritional mixture with (TRP+) or without TRP (TRP-) or after no treatment at all.

\begin{tabular}{|c|c|c|c|}
\hline \multirow[t]{2}{*}{ LNAA } & \multirow[t]{2}{*}{ Treatment } & \multicolumn{2}{|l|}{ Time } \\
\hline & & Baseline & $\mathrm{t} 240$ \\
\hline \multirow[t]{4}{*}{ TRP } & no treatment & $80 \pm 9$ & $76 \pm 11$ \\
\hline & saline & $107 \pm 12$ & $77 \pm 8$ \\
\hline & TRP+ & $107 \pm 9$ & $97 \pm 8$ \\
\hline & TRP- & $104 \pm 13$ & $43 \pm 4 *$ \\
\hline \multirow[t]{4}{*}{ TYR } & no treatment & $76 \pm 3$ & $70 \pm 3$ \\
\hline & saline & $84 \pm 7$ & $66 \pm 3$ \\
\hline & TRP+ & $92 \pm 4$ & $42 \pm 3^{\#}$ \\
\hline & TRP- & $90 \pm 4$ & $45 \pm 2$ \\
\hline \multirow[t]{4}{*}{ PHE } & no treatment & $67 \pm 1$ & $90 \pm 8$ \\
\hline & saline & $77 \pm 7$ & $76 \pm 4$ \\
\hline & TRP+ & $75 \pm 4$ & $90 \pm 5$ \\
\hline & TRP- & $73 \pm 3$ & $96 \pm 5$ \\
\hline \multirow[t]{4}{*}{ VAL } & no treatment & $147 \pm 6$ & $177 \pm 5$ \\
\hline & saline & $166 \pm 15$ & $179 \pm 6$ \\
\hline & TRP+ & $165 \pm 8$ & $185 \pm 5$ \\
\hline & TRP- & $159 \pm 1$ & $205 \pm 6$ \\
\hline \multirow[t]{4}{*}{ ILE } & no treatment & $124 \pm 6$ & $168 \pm 14$ \\
\hline & saline & $136 \pm 14$ & $168 \pm 7$ \\
\hline & TRP+ & $123 \pm 5$ & $104 \pm 5^{\#}$ \\
\hline & TRP- & $127 \pm 2$ & $118 \pm 8$ \\
\hline \multirow[t]{4}{*}{ LEU } & no treatment & $162 \pm 7$ & $196 \pm 10$ \\
\hline & saline & $186 \pm 17$ & $208 \pm 6$ \\
\hline & TRP+ & $170 \pm 7$ & $164 \pm 4^{\#}$ \\
\hline & TRP- & $175 \pm 2$ & $179 \pm 6$ \\
\hline
\end{tabular}

$(\#)$ TRP+ group significantly different from saline; $(*)$ TRP- group significantly different from TRP+ group. (\#,*) $P<0.017$. TRP: tryptophan, TYR: tyrosine, PHE: phenylalanine, VAL: valine, ILE: isoleucine, LEU: leucine 
No differences were found between the TRP+ and the saline group. Separate one-way ANOVAs to reveal treatment effects per time-point showed no differences at base-line $[F(3,39)=0.998$; n.s.]. However, a significant treatment effect was found $4 \mathrm{~h}$ after the first treatment administration $[F(3,37)=7.92$; $P<0.001]$. Post hoc multiple comparisons revealed that the ratio TRP/ $\Sigma L N A A$ in plasma was significantly lower after TRP- $[t(16)=5.525 ; P<0.017]$ and saline treatment $[t(18)=-2.912 ; P<0.017]$ compared to TRP+ treatment. No differences were found between the saline group and the group that received no treatment at all $[t(18)=-0.082 ;$ n.s. $]$.

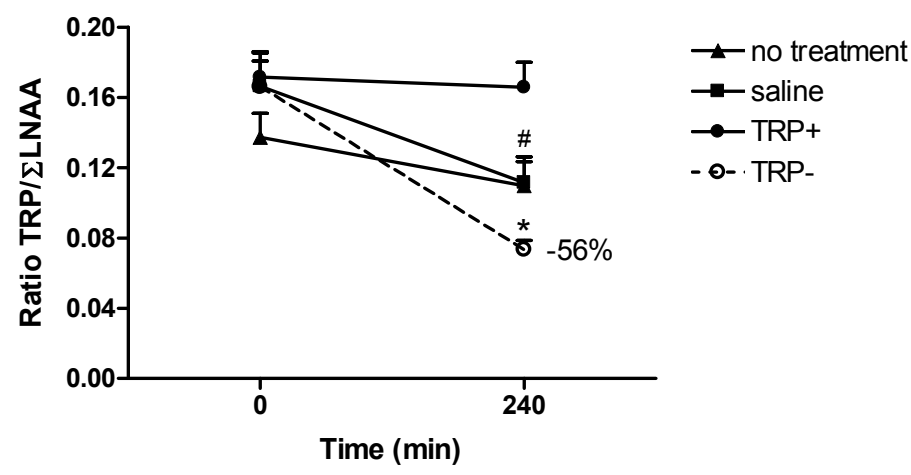

Figure 1 The ratio TRP/ $\Sigma$ LNAA in plasma (mean + S.E.M.) at baseline and 240 min after the first oral administration with either saline or the protein-carbohydrate nutritional mixture with (TRP+) or without TRP (TRP-) or after no treatment at all. The group that received no treatment at all did not differ from the saline group. Significant decreases were found $4 \mathrm{~h}$ after treatment with TRP- $(*)$ and saline $\left(^{\#}\right)$ compared to TRP+ treatment. $* / \# P<0.017$. Percentage indicates mean change from corresponding baseline levels after ATD treatment.

\section{Brain amino acids, 5-HT, 5-HIAA and DA}

Table 2 shows the concentrations of central amino acids and the monoamines 5-HT and DA, 5-HIAA and 5-HT turnover rate (5-HIAA/5-HT).

Whereas ATD significantly decreased the TRP levels in plasma, no ATD effects were found upon central TRP concentrations in the hippocampus $[t(16)$ $=1.213$; n.s.] or prefrontal cortex $[t(15)=0.744$; n.s.]. In the hippocampus, a significant decrease of both 5-HIAA concentrations $(-33 \%)[t(16)=3.384$; $P<0.017]$ and the 5-HT turnover $(-28 \%)[t(14)=3.034 ; P<0.017]$ were observed $4 \mathrm{~h}$ after ATD compared to the TRP+ control group. Other treatment effects were only found between the TRP+ and saline control groups. TRP + treatment decreased the TYR levels in the hippocampus $[t(18)=$ 4.627; $P<P<0.017]$ and prefrontal cortex $[t(18)=3.468 ; P<0.017]$. This was also the case for the concentration of ILE with decreased levels in both the 
hippocampus $[t(18)=5.184 ; P<0.017]$ and the prefrontal cortex $[t(17)=3.725$; $P<0.017]$. Further, TRP+ treatment increased the concentration of VAL in the hippocampus $[t(16)=-2.690 ; P<0.017]$. No differences were found upon any of the parameters measured between the group that did not receive any treatment and the saline control group.

Table 2 Absolute LNAAs, 5-HT, 5-HIAA, 5-HT turnover and DA concentrations (mean \pm S.E.M. in $\mathrm{ng} / \mathrm{mg}$ ) in rat hippocampus and prefrontal cortex, $240 \mathrm{~min}$ after the first oral administration with either saline or the protein-carbohydrate nutritional mixture with $(\mathrm{TRP}+)$ or without TRP (TRP-) or after no treatment at all.

\begin{tabular}{|c|c|c|c|}
\hline & Treatment & Brain tissue & \\
\hline & & Hippocampus & Prefrontal cortex \\
\hline \multirow[t]{4}{*}{ TRP } & no treatment & $9.30 \pm 0.28$ & $13.60 \pm 0.60$ \\
\hline & saline & $8.73 \pm 0.52$ & $13.40 \pm 0.85$ \\
\hline & TRP+ & $8.37 \pm 0.57$ & $12.62 \pm 1.18$ \\
\hline & TRP- & $7.51 \pm 0.33$ & $11.66 \pm 0.34$ \\
\hline \multirow[t]{4}{*}{ TYR } & no treatment & $10.83 \pm 0.38$ & $11.98 \pm 1.19$ \\
\hline & saline & $9.79 \pm 0.49$ & $10.99 \pm 0.49$ \\
\hline & TRP+ & $7.14 \pm 0.30 \#$ & $8.39 \pm 0.57 \#$ \\
\hline & TRP- & $7.85 \pm 0.38$ & $8.41 \pm 0.24$ \\
\hline \multirow[t]{4}{*}{ PHE } & no treatment & $9.81 \pm 0.37$ & $11.95 \pm 0.38$ \\
\hline & saline & $9.10 \pm 0.42$ & $11.99 \pm 0.50$ \\
\hline & TRP+ & $10.98 \pm 0.60$ & $13.41 \pm 0.89$ \\
\hline & TRP- & $11.74 \pm 0.53$ & $14.18 \pm 0.32$ \\
\hline \multirow[t]{4}{*}{ VAL } & no treatment & $13.97 \pm 0.48$ & $14.59 \pm 0.55$ \\
\hline & saline & $13.29 \pm 0.30$ & $14.53 \pm 0.56$ \\
\hline & TRP+ & $15.47 \pm 0.68 \#$ & $16.65 \pm 0.91$ \\
\hline & TRP- & $17.69 \pm 0.70$ & $18.06 \pm 0.64$ \\
\hline \multirow[t]{4}{*}{ ILE } & no treatment & $7.36 \pm 0.22$ & $9.21 \pm 0.42$ \\
\hline & saline & $7.00 \pm 0.23$ & $8.79 \pm 0.33$ \\
\hline & TRP+ & $5.34 \pm 0.22 \#$ & $6.63 \pm 0.49 \#$ \\
\hline & TRP- & $5.95 \pm 0.24$ & $7.40 \pm 0.16$ \\
\hline \multirow[t]{4}{*}{ LEU } & no treatment & $11.63 \pm 0.37$ & $12.52 \pm 0.41$ \\
\hline & saline & $11.58 \pm 0.25$ & $12.41 \pm 0.33$ \\
\hline & TRP+ & $10.70 \pm 0.31$ & $11.71 \pm 0.39$ \\
\hline & TRP- & $11.71 \pm 0.41$ & $12.81 \pm 0.44$ \\
\hline
\end{tabular}




\begin{tabular}{llll}
\hline & Treatment & Brain tissue & \\
\hline 5-HT & Hippocampus & Prefrontal cortex \\
& no treatment & $0.22 \pm 0.01$ & $0.43 \pm 0.05$ \\
saline & $0.23 \pm 0.01$ & $0.45 \pm 0.06$ \\
TRP+ & $0.24 \pm 0.02$ & $0.38 \pm 0.07$ \\
TRP- & $0.20 \pm 0.01$ & $0.39 \pm 0.04$ \\
& no treatment & $0.17 \pm 0.01$ & $0.20 \pm 0.01$ \\
saline & $0.17 \pm 0.01$ & $0.22 \pm 0.01$ \\
TRPA & $0.15 \pm 0.01$ & $0.20 \pm 0.02$ \\
& TRP- & $0.10 \pm 0.01 *$ & $0.18 \pm 0.02$ \\
no treatment & $0.78 \pm 0.05$ & $0.49 \pm 0.05$ \\
& saline & $0.75 \pm 0.06$ & $0.51 \pm 0.06$ \\
& TRP+ & $0.71 \pm 0.05$ & $0.68 \pm 0.12$ \\
& TRP- & $0.51 \pm 0.03 *$ & $0.53 \pm 0.10$ \\
no treatment & $0.61 \pm 0.04$ & $0.23 \pm 0.02$ \\
& saline & $0.67 \pm 0.02$ & $0.22 \pm 0.02$ \\
TRP+ & TRP- & $0.71 \pm 0.12$ & $0.22 \pm 0.02$ \\
& $0.80 \pm 0.07$ & $0.21 \pm 0.02$ \\
\hline
\end{tabular}

(\#) TRP+ group significantly different from saline; $(*)$ TRP- group significantly different from TRP+ group. (\#,*) $P<0.017$. LNAAs: large neutral amino acids, TRP: tryptophan, TYR: tyrosine, PHE: phenylalanine, VAL: valine, ILE: isoleucine, LEU: leucine, 5-HT: serotonin, 5-HIAA: 5hydroxyindolacetic acid, 5-HT turnover: 5-HIAA/5-HT, DA: dopamine.

\section{Serum and central BDNF}

Serum BDNF levels (Fig. 2$)$ did not change over time $[F(1,32)=3.84$; n.s.] and no overall treatment effect was found $[F(3,32)=0.51$; n.s.]. Separate one-way ANOVAs to reveal treatment effects per time-point showed that no differences in serum BDNF levels between the treatment conditions could be observed at baseline $[F(3,39)=0.50$; n.s. $]$ or $4 \mathrm{~h}$ after the first oral administration $[F(3,35)$ $=1.08 ;$ n.s.].

ATD did not affect BDNF levels in the hippocampus $[t(15)=0.140$; n.s. $]$ or the prefrontal cortex $[t(15)=0.672 ;$ n.s. $]$ compared to the TRP + group (Fig. 3). No differences in BDNF levels were found between the TRP+ and saline control groups in hippocampus $[t(18)=0.608$; n.s. $]$ or prefrontal cortex $[t(14)=$ -0.320 ; n.s.]. Saline treatment compared to the group that did not receive any treatment at all significantly decreased the BDNF levels in the prefrontal cortex 
$[t(13)=4.210 ; P<0.017]$ without affecting hippocampal BDNF levels $[t(16)=$ $-0.031 ;$ n.s. $]$.

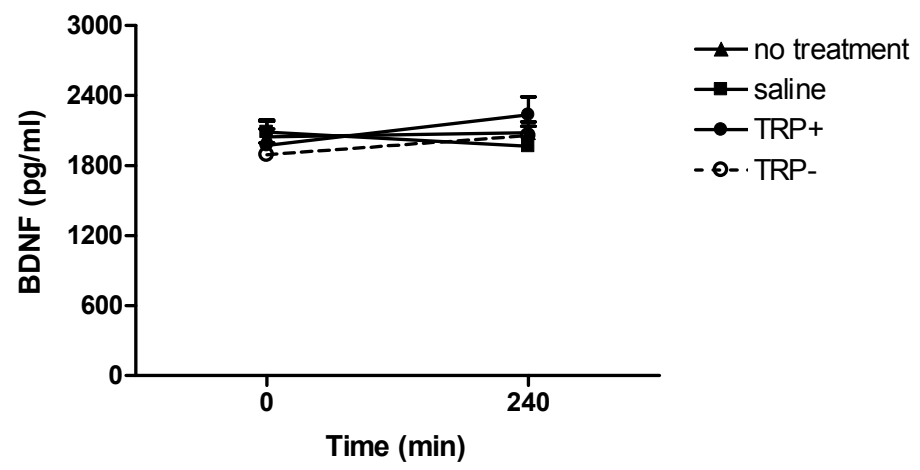

Figure 2 Serum BDNF levels (mean + S.E.M. in $\mathrm{pg} / \mathrm{ml}$ ) at baseline and $240 \mathrm{~min}$ after the first oral administration with either saline or the protein-carbohydrate nutritional mixture with (TRP+) or without TRP (TRP) or after no treatment at all. ATD did not affect serum BDNF levels.

\section{Correlations}

Correlation analysis was performed to reveal significant associations between peripheral and central parameters as depicted in Table 3. Absolute plasma TRP levels and the ratio TRP $/ \Sigma$ LNAA were highly correlated $\left(r_{\mathrm{p}}=0.94, P<0.05\right)$. Positive associations were also found between peripheral TRP levels and 5-HTrelated parameters in the hippocampus, not, however, in the prefrontal cortex. Only in the brain, TRP concentrations correlated with 5-HT itself in both the hippocampus $\left(r_{\mathrm{p}}=0.31, P<0.05\right)$ and prefrontal cortex $\left(r_{\mathrm{p}}=0.46, P<0.05\right)$. A highly mutual pattern of positive correlations was also observed between the 5-HT related parameters within the hippocampus.

Serum BDNF did not significantly correlate with hippocampal BDNF levels $\left(r_{\mathrm{p}}=0.01, P=0.97\right)$ or BDNF levels in the prefrontal cortex $\left(r_{\mathrm{p}}=0.25, P=0.19\right)$. Although ATD did not significantly affect the central BDNF levels, a positive correlation was found between both TRP and BDNF in the hippocampus $\left(r_{\mathrm{p}}=\right.$ $0.37, P<0.05)$ and TRP and BDNF in the prefrontal cortex $\left(r_{\mathrm{p}}=0.42, P<0.05\right)$. 


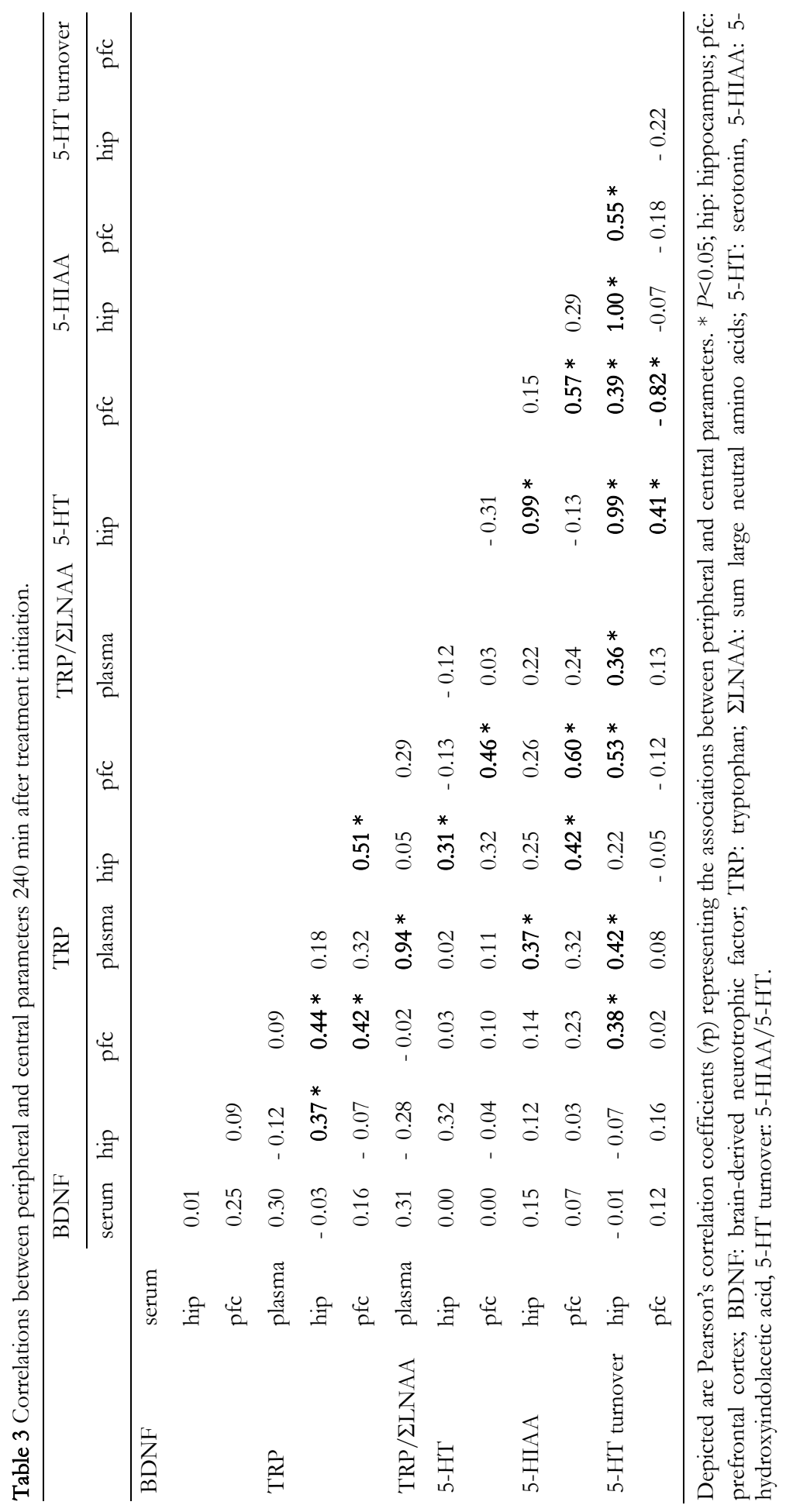




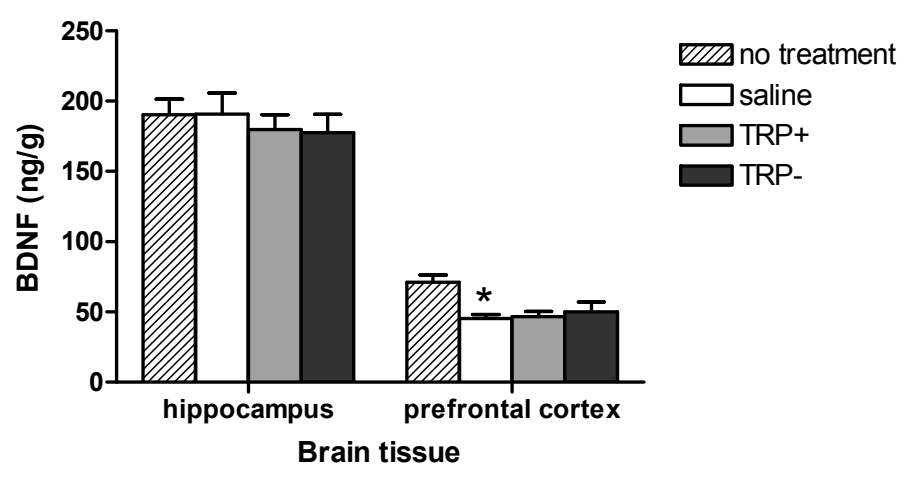

Figure 3 Central BDNF concentrations in hippocampus and prefrontal cortex (mean + S.E.M. in $\mathrm{ng} / \mathrm{g}$ ), $240 \mathrm{~min}$ after the first oral administration with either saline or the protein-carbohydrate nutritional mixture with (TRP+) or without TRP (TRP-) or after no treatment at all. $* P<0.017$; BDNF levels were significantly decreased in the prefrontal cortex after saline treatment compared to the group that received no treatment at all. No ATD effects (TRP+ compared to TRP-) were found upon BDNF concentration and no differences were found between the TRP+ and saline treatment groups.

\section{Discussion}

In the present study, ATD was applied to further explore the underlying mechanism of the serotonergic challenge tool by investigating the relationship between the 5-HT system and BDNF protein levels. Furthermore, effects of experimental stress upon BDNF concentrations were measured as a potential confounding factor for ATD-induced functional and behavioural alterations in the rat. ATD was accomplished by administering a TRP-free proteincarbohydrate nutritional mixture to measure the effects of an acute change in peripheral TRP, the dietary 5-HT precursor, upon BDNF protein levels in serum and rat hippocampus and prefrontal cortex. Whereas $4 \mathrm{~h}$ after ATD treatment initiation, the ratio TRP/ $/ \mathrm{LNAA}$ in plasma was decreased with $56 \%$, no direct ATD effects were observed upon absolute TRP or 5-HT levels in the brain at this specific time-point. However, both the concentration of the 5-HT metabolite, 5-HIAA, and the 5-HT turnover rate were significantly decreased in the hippocampus, indicative of an ATD-induced diminished 5-HT metabolism in this brain region. Although ATD did not directly alter peripheral or central BDNF levels $4 \mathrm{~h}$ after treatment initiation, a positive correlation was found between TRP concentrations and BDNF levels at this time-point in both the hippocampus and the prefrontal cortex. Moreover, a decrease in BDNF levels was observed in the prefrontal cortex after oral application by gavage, thereby 
confirming our hypothesis that experimental stressors decrease basal BDNF levels and possibly interfere with ATD neurochemical effects.

The ATD challenge method is extensively applied in both clinical and preclinical research to explore the implication of the 5-HT system in a variety of cognitive and affective disorders. However, the exact underlying mechanism through which it exerts its neurophysiological effect is still poorly understood. Additionally, it remains unclear to what extend ATD-induced cognitive dysfunctions and affective behavioural alterations are due to actual changes in 5-HT neuronal activity. In the present study, ATD substantially decreased peripheral TRP levels and the ratio TRP/ $/ \mathrm{LNAA} 4 \mathrm{~h}$ after treatment initiation in line with other studies of similar experimental design (Jans et al., 2007a; Jans and Blokland, 2008; Lieben et al., 2004a; Olivier et al., 2008). The lower TRP/ $\Sigma$ LNAA ratio in plasma after both saline and no treatment compared to TRP + treatment can be ascribed to the lack of nutrients to maintain an optimal level of blood amino acid concentrations. Additionally, these results provide evidence that the boost of large neutral amino acids in the absence of TRP is the key factor for depleting TRP in plasma, as the TRP-treatment group was the only group that decreased the ratio significantly from baseline levels. Nevertheless, no direct ATD effects upon central TRP or 5-HT levels were found at this time-point. However, the reduction of hippocampal 5-HIAA suggests that less 5-HT is broken down into its inactive metabolite, presumably caused by a reduced availability of the precursor itself (Fernstrom and Hirsch, 1977). This is also reflected in the significant decrease of the 5-HT turnover rate, i.e. the ratio 5-HIAA/5-HT. Both decreased concentrations of 5-HIAA and a decrease in the ratio of the concentrations of 5-HIAA to 5-HT are valid indices of reduced 5-HT metabolism (Shannon et al., 1986). Since dietary precursor manipulation was applied, this reduced 5-HT metabolism could be fully ascribed to a reduction in precursor availability or even to a reduction in the monoamine oxidase enzyme (MAO), thereby reducing the catabolic rate (Fernstrom and Hirsch, 1977). The latter would provide an explanation for the fact that 5-HT turnover reduces whereas 5-HT levels remain unchanged, as found in the present study.

A strong negative association was observed between 5-HT and 5-HT turnover in the prefrontal cortex, without any correlations between 5-HT turnover and 5-HIAA concentrations. It, thus, seems that ATD-induced biochemical alterations are limited to the hippocampus, which would corroborate the notion that the method is reliable and relatively stable for its cognitive dysfunctional effects in both human subjects and rats. Impaired memory consolidation processes after ATD in healthy subjects (Riedel et al., 1999) have been generally attributed to decreased 5-HT in hippocampal areas (Riedel, 2004). Studies of similar ATD experimental design, using the same nutritional mixture and dosing regime, consistently report profound object memory impairments in rats $4 \mathrm{~h}$ after the first oral administration, as measured by the object recognition task (Jans et al., 2007a; Jans and Blokland, 2008; 
Lieben et al., 2004b; Olivier et al., 2008). Further, even a moderate depletion of TRP in plasma (Rutten et al., 2007) and single dosing within a relatively short period of time (van Donkelaar et al., 2008) considerably interfered with optimal object recognition performance and thus the ATD method can be considered a reliable memory deficit model. Since BDNF is required for specific memory processes (Lee et al., 2004), the positive associations found between central TRP and BDNF in the present study are in favour of the suggestion that ATDinduced cognitive dysfunctional behaviour is BDNF-mediated.

In addition, higher concentrations of TRP, 5-HT and 5-HIAA can be observed in the prefrontal cortex compared to the hippocampus. This could provide an explanation for the fact that, under normal physiological conditions, a considerable manipulation of the dietary precursor does not affect the TRP concentration sufficiently to alter 5-HT synthesis and metabolism in this brain area. In other words, enough TRP will remain available to maintain the ratelimiting enzyme TPH saturated and the rate of 5-HT synthesis unmodified (Boadle-Biber, 1993), which is in line with the fact that ATD only has a moodlowering effect in so-called serotonergic vulnerable subjects due to pre-existing 5-HT dysfunction (Booij et al., 2002; Jans et al., 2007b).

Given that disrupted 5-HT metabolism does not unconditionally imply diminished 5-HT neuronal activity, the underlying mechanism of the ATDinduced behavioural changes might go beyond alterations in the 5-HT system. The pivotal role of disrupted BDNF regulation in both depressive symptomatology and cognitive dysfunction, as well as the co-regulating mechanisms between BDNF and the 5-HT system in general, led us to explore the effects of ATD upon peripheral and central BDNF levels $4 \mathrm{~h}$ after treatment initiation. In line with a previous study, which measured ATDinduced changes in BDNF plasma levels (Cahir et al., 2008), ATD in the present study did not affect BDNF levels in serum or central BDNF concentrations in the hippocampus or prefrontal cortex. We measured serum $\mathrm{BDNF}$, since BDNF is released into the plasma through platelet activation or clotting processes (Fujimura et al., 2002). However, it has been suggested that changes in serum BNDF might only reflect an in-vitro-effect of alterations in platelet release (Cahir et al., 2008; Karege et al., 2005). Yet, since BDNF in whole blood is essentially stored in blood platelets, much higher concentrations of BNDF are found in serum than in plasma (Radka et al., 1996). Although it is generally accepted that neurotrophins, including BDNF, do not easily cross the blood-brain barrier in vivo (Pardridge, 2002), evidence exists that BDNF is transported into the brain by a saturable transport mechanism (Pan et al., 1998). It, therefore, has been suggested that peripheral changes might reflect central processes. However, this is not supported by the results of the present study as BDNF in serum did not correlate with BNDF levels in the hippocampus or prefrontal cortex. Yet, positive correlations have been found between plasma $\mathrm{BDNF}$ and $\mathrm{BNDF}$ concentrations in the dorsal raphe/periaqueductal grey region of the brain (Cahir et al., 2008). 
Whereas no differences were observed in central BDNF protein levels between the experimental groups that received either saline or the nutritional mixture with (TRP+ group) or without TRP (TRP- group), a significant decrease in BDNF levels was found in the prefrontal cortex of the saline treated rats compared to the group that did not receive any treatment at all. Although blood sampling procedures were similar between all groups, the latter was not deprived of food overnight, retained free access to food throughout the entire experiment and did not receive any oral applications by gavage. Overnight food deprivation and oral gavage procedures can be seen as considerably stressful and might interfere with normal brain TRP metabolism (Curzon et al., 1972). Both acute and repeated exposures to stressful stimuli have been shown to increase glucocorticoid levels and alter 5-HT turnover and release in both the hippocampus and frontal cortex (Chaouloff, 1993). Acute and repeated stress decrease BDNF mRNA in the hippocampus within $1 \mathrm{~h}$ (Smith et al., 1995b), partly due to the negative feedback of increased corticosterone levels. Thus, a stress-related decrease in prefrontal BDNF might change 5-HT and its associated parameters in all groups similarly, thereby interfering with ATDinduced alterations in 5-HT metabolism. Stress-induced changes in BDNF might have interfered with the ATD-induced alterations in 5-HT metabolism in general and in the prefrontal cortex in particular. Further studies are needed to elucidate the direct effect of an acute decrease in central BDNF levels upon 5HT metabolism in the brain.

In conclusion, although ATD did not directly affect serum or central BDNF levels, positive correlations were found between TRP and BDNF in both the hippocampus and prefrontal cortex. The ATD-induced changes in 5-HT metabolism might not have been sufficient to cause significant alterations in peripheral and central BDNF protein levels. Moreover, other factors influencing BDNF, including experimental stressors, might have interfered with 5-HT metabolism in general, thereby preventing or changing the expected ATD-induced neurochemical alterations. Nevertheless, the positive relationship between TRP and BDNF levels in the brain found in the present study suggests that central BDNF is linked to TRP. Although this supports the speculation that ATD-induced rat object memory impairment in particular and dysfunctional cognitive behaviour in general might be hippocampal BDNFmediated, ATD application-related stress affecting prefrontal BDNF seems involved in this respect as well.

\section{Acknowledgements}

The authors would like to thank Marianne Markerink-Van Ittersum and Denise Hermes for their excellent technical assistance and realization of the ELISAs. 


\section{References}

Altar CA, Whitehead RE, Chen R, Wortwein G, Madsen TM (2003). Effects of electroconvulsive seizures and antidepressant drugs on brain-derived neurotrophic factor protein in rat brain. Biol Psychiatry 54(7): 703-709.

Bel N, Artigas F (1996). Reduction of serotonergic function in rat brain by tryptophan depletion: effects in control and fluvoxamine-treated rats. J Neurochem 67(2): 669-676.

Bell CJ, Hood SD, Nutt DJ (2005). Acute tryptophan depletion. Part II: clinical effects and implications. Aust N Z J Psychiatry 39(7): 565-574.

Bliss TV, Collingridge GL (1993). A synaptic model of memory: long-term potentiation in the hippocampus. Nature 361(6407): 31-39.

Blokland A, Lieben C, Deutz NEP, Schmitt J (2004). Acute Tryptophan depletion: comparing the effects of an amino acid mixture with a gelatin-based protein in man and rats. Current Topics in Nutraceutical Research 2(3): 1-8.

Boadle-Biber MC (1993). Regulation of serotonin synthesis. Prog Biophys Mol Biol 60(1): 1-15.

Booij L, Van der Does W, Benkelfat C, Bremner JD, Cowen PJ, Fava M, Gillin C, Leyton M, Moore P, Smith KA, Van der Kloot WA (2002). Predictors of mood response to acute tryptophan depletion. A reanalysis. Neuropsychopharmacology 27(5): 852-861.

Booij L, Van der Does AJ, Riedel WJ (2003). Monoamine depletion in psychiatric and healthy populations: review. Mol Psychiatry 8(12): 951-973.

Cahir M, Ardis TC, Elliott JJ, Kelly CB, Reynolds GP, Cooper SJ (2008). Acute tryptophan depletion does not alter central or plasma brain-derived neurotrophic factor in the rat. Eur Neuropsychopharmacol 18(5): 317-322.

Carpenter LL, Anderson GM, Pelton GH, Gudin JA, Kirwin PD, Price LH, Heninger GR, McDougle CJ (1998). Tryptophan depletion during continuous CSF sampling in healthy human subjects. Neuropsychopharmacology 19(1): 26-35.

Chaouloff F (1993). Physiopharmacological interactions between stress hormones and central serotonergic systems. Brain Res Brain Res Rev 18(1): 1-32.

Conner JM, Lauterborn JC, Yan Q, Gall CM, Varon S (1997). Distribution of brain-derived neurotrophic factor $(\mathrm{BDNF})$ protein and $\mathrm{mRNA}$ in the normal adult rat CNS: evidence for anterograde axonal transport. J Neurosci 17(7): 2295-2313.

Croll SD, Wiegand SJ, Anderson KD, Lindsay RM, Nawa H (1994). Regulation of neuropeptides in adult rat forebrain by the neurotrophins BDNF and NGF. Eur J Neurosci 6(8): 13431353.

Curzon G, Joseph MH, Knott PJ (1972). Effects of immobilization and food deprivation on rat brain tryptophan metabolism. J Neurochem 19(8): 1967-1974.

Dixon WJ (1959). Analysis of extreme values. Ann Math Stat 21: 488-506.

Duman RS, Heninger GR, Nestler EJ (1997). A molecular and cellular theory of depression. Arch Gen Psychiatry 54(7): 597-606.

Duman RS (1998). Novel therapeutic approaches beyond the serotonin receptor. Biol Psychiatry 44(5): 324-335.

Ernfors P, Wetmore C, Olson L, Persson H (1990). Identification of cells in rat brain and peripheral tissues expressing mRNA for members of the nerve growth factor family. Neuron 5(4): 511-526.

Fadda F, Cocco S, Rossetti ZL, Melis G, Stancampiano R (2000a). A tryptophan-free diet markedly reduces frontocortical 5-HT release, but fails to modify ethanol preference in alcohol-preferring (sP) and non-preferring (sNP) rats. Behav Brain Res 108(2): 127-132.

Fadda F, Cocco S, Stancampiano R (2000b). A physiological method to selectively decrease brain serotonin release. Brain Res Brain Res Protoc 5(3): 219-222.

Fernstrom JD, Wurtman RJ (1972). Brain serotonin content: physiological regulation by plasma neutral amino acids. Science 178(59): 414-416.

Fernstrom JD, Hirsch MJ (1977). Brain serotonin synthesis: reduction in corn-malnourished rats. J Neurochem 28(4): 877-979. 
Fernstrom JD (1979). Diet-induced changes in plasma amino acid pattern: effects on the brain uptake of large neutral amino acids, and on brain serotonin synthesis. J Neural Transm Suppl(15): 55-67.

Fernstrom JD (1983). Role of precursor availability in control of monoamine biosynthesis in brain. Physiol Rev 63(2): 484-546.

Fernstrom JD (1986). Acute and chronic effects of protein and carbohydrate ingestion on brain tryptophan levels and serotonin synthesis. Nutr Rev 44 Suppl: 25-36.

Figurov A, Pozzo-Miller LD, Olafsson P, Wang T, Lu B (1996). Regulation of synaptic responses to high-frequency stimulation and LTP by neurotrophins in the hippocampus. Nature 381(6584): 706-709.

Fujimura H, Altar CA, Chen R, Nakamura T, Nakahashi T, Kambayashi J, Sun B, Tandon NN (2002). Brain-derived neurotrophic factor is stored in human platelets and released by agonist stimulation. Thromb Haemost 87(4): 728-734.

Ghosh A, Carnahan J, Greenberg ME (1994). Requirement for BDNF in activity-dependent survival of cortical neurons. Science 263(5153): 1618-1623.

Gonul AS, Akdeniz F, Taneli F, Donat O, Eker C, Vahip S (2005). Effect of treatment on serum brain-derived neurotrophic factor levels in depressed patients. Eur Arch Psychiatry Clin Neurosci 255(6): 381-386.

Hem A, Smith AJ, Solberg P (1998). Saphenous vein puncture for blood sampling of the mouse, rat, hamster, gerbil, guinea pig, ferret and mink. Lab Anim 32(4): 364-368.

Hicks RR, Martin VB, Zhang L, Seroogy KB (1999). Mild experimental brain injury differentially alters the expression of neurotrophin and neurotrophin receptor mRNAs in the hippocampus. Exp Neurol 160(2): 469-478.

Hofer M, Pagliusi SR, Hohn A, Leibrock J, Barde YA (1990). Regional distribution of brainderived neurotrophic factor mRNA in the adult mouse brain. Embo J 9(8): 2459-2464.

Hood SD, Bell CJ, Nutt DJ (2005). Acute tryptophan depletion. Part I: rationale and methodology. The Australian and New Zealand journal of psychiatry 39(7): 558-564.

Jans LA, Lieben CK, Blokland A (2007a). Influence of sex and estrous cycle on the effects of acute tryptophan depletion induced by a gelatin-based mixture in adult Wistar rats. Neuroscience 147(2): 304-317.

Jans LA, Riedel WJ, Markus CR, Blokland A (2007b). Serotonergic vulnerability and depression: assumptions, experimental evidence and implications. Mol Psychiatry 12(6): 522-543.

Jans LA, Blokland A (2008). Influence of chronic mild stress on the behavioural effects of acute tryptophan depletion induced by a gelatin-based mixture. Behav Pharmacol 19(7): 706-715.

Karege F, Bondolfi G, Gervasoni N, Schwald M, Aubry JM, Bertschy G (2005). Low brainderived neurotrophic factor (BDNF) levels in serum of depressed patients probably results from lowered platelet BDNF release unrelated to platelet reactivity. Biol Psychiatry 57(9): 1068-1072.

Klaassen T, Riedel WJ, van Someren A, Deutz NE, Honig A, van Praag HM (1999). Mood effects of 24-hour tryptophan depletion in healthy first-degree relatives of patients with affective disorders. Biol Psychiatry 46(4): 489-497.

Korte M, Carroll P, Wolf E, Brem G, Thoenen H, Bonhoeffer T (1995). Hippocampal long-term potentiation is impaired in mice lacking brain-derived neurotrophic factor. Proc Natl Acad Sci U S A 92(19): 8856-8860.

Lee JL, Everitt BJ, Thomas KL (2004). Independent cellular processes for hippocampal memory consolidation and reconsolidation. Science 304(5672): 839-843.

Lieben CK, Blokland A, Westerink B, Deutz NE (2004a). Acute tryptophan and serotonin depletion using an optimized tryptophan-free protein-carbohydrate mixture in the adult rat. Neurochem Int 44(1): 9-16.

Lieben CK, van Oorsouw K, Deutz NE, Blokland A (2004b). Acute tryptophan depletion induced by a gelatin-based mixture impairs object memory but not affective behavior and spatial learning in the rat. Behav Brain Res 151(1-2): 53-64.

Lieben CK, Blokland A, Sik A, Sung E, van Nieuwenhuizen P, Schreiber R (2005). The selective 5-HT6 receptor antagonist Ro4368554 restores memory performance in cholinergic and 
serotonergic models of memory deficiency in the rat. Neuropsychopharmacology 30(12): 2169-2179.

Mamounas LA, Blue ME, Siuciak JA, Altar CA (1995). Brain-derived neurotrophic factor promotes the survival and sprouting of serotonergic axons in rat brain. J Neurosci 15(12): 7929-7939.

Mamounas LA, Altar CA, Blue ME, Kaplan DR, Tessarollo L, Lyons WE (2000). BDNF promotes the regenerative sprouting, but not survival, of injured serotonergic axons in the adult rat brain. J Neurosci 20(2): 771-782.

Markus CR, Panhuysen G, Tuiten A, Koppeschaar H, Fekkes D, Peters ML (1998). Does carbohydrate-rich, protein-poor food prevent a deterioration of mood and cognitive performance of stress-prone subjects when subjected to a stressful task? Appetite 31(1): 4965.

Mattson MP, Maudsley S, Martin B (2004). BDNF and 5-HT: a dynamic duo in age-related neuronal plasticity and neurodegenerative disorders. Trends Neurosci 27(10): 589-594.

McAllister AK, Lo DC, Katz LC (1995). Neurotrophins regulate dendritic growth in developing visual cortex. Neuron 15(4): 791-803.

Moore P, Landolt HP, Seifritz E, Clark C, Bhatti T, Kelsoe J, Rapaport M, Gillin JC (2000). Clinical and physiological consequences of rapid tryptophan depletion. Neuropsychopharmacology 23(6): 601-622.

Nawa H, Pelleymounter MA, Carnahan J (1994). Intraventricular administration of BDNF increases neuropeptide expression in newborn rat brain. J Neurosci 14(6): 3751-3765.

Neumeister A, Konstantinidis A, Stastny J, Schwarz MJ, Vitouch O, Willeit M, Praschak-Rieder N, Zach J, de Zwaan M, Bondy B, Ackenheil M, Kasper S (2002). Association between serotonin transporter gene promoter polymorphism (5HTTLPR) and behavioral responses to tryptophan depletion in healthy women with and without family history of depression. Arch Gen Psychiatry 59(7): 613-620.

Neumeister A (2003). Tryptophan depletion, serotonin, and depression: where do we stand? Psychopharmacol Bull 37(4): 99-115.

Neumeister A, Yuan P, Young TA, Bonne O, Luckenbaugh DA, Charney DS, Manji H (2005). Effects of tryptophan depletion on serum levels of brain-derived neurotrophic factor in unmedicated patients with remitted depression and healthy subjects. Am J Psychiatry 162(4): 805-807.

Neumeister A, Hu XZ, Luckenbaugh DA, Schwarz M, Nugent AC, Bonne O, Herscovitch P, Goldman D, Drevets WC, Charney DS (2006). Differential effects of 5-HTTLPR genotypes on the behavioral and neural responses to tryptophan depletion in patients with major depression and controls. Arch Gen Psychiatry 63(9): 978-986.

Nibuya M, Morinobu S, Duman RS (1995). Regulation of BDNF and trkB mRNA in rat brain by chronic electroconvulsive seizure and antidepressant drug treatments. J Neurosci 15(11): $7539-7547$.

Nibuya M, Nestler EJ, Duman RS (1996). Chronic antidepressant administration increases the expression of cAMP response element binding protein (CREB) in rat hippocampus. J Neurosci 16(7): 2365-2372.

Olivier JD, Jans LA, Korte-Bouws GA, Korte SM, Deen PM, Cools AR, Ellenbroek BA, Blokland A (2008). Acute tryptophan depletion dose dependently impairs object memory in serotonin transporter knockout rats. Psychopharmacology (Berl) 200(2): 243-254.

Pan W, Banks WA, Fasold MB, Bluth J, Kastin AJ (1998). Transport of brain-derived neurotrophic factor across the blood-brain barrier. Neuropharmacology 37(12): 1553-1561.

Pardridge WM (2002). Blood-brain barrier drug targeting enables neuroprotection in brain ischemia following delayed intravenous administration of neurotrophins. Adv Exp Med Biol 513: 397-430.

Prickaerts J, van den Hove DL, Fierens FL, Kia HK, Lenaerts I, Steckler T (2006). Chronic corticosterone manipulations in mice affect brain cell proliferation rates, but only partly affect BDNF protein levels. Neurosci Lett 396(1): 12-16. 
Radka SF, Holst PA, Fritsche M, Altar CA (1996). Presence of brain-derived neurotrophic factor in brain and human and rat but not mouse serum detected by a sensitive and specific immunoassay. Brain Res 709(1): 122-301.

Riedel WJ, Klaassen T, Deutz NE, van Someren A, van Praag HM (1999). Tryptophan depletion in normal volunteers produces selective impairment in memory consolidation. Psychopharmacology (Berl) 141(4): 362-369.

Riedel WJ (2004). Cognitive changes after acute tryptophan depletion: what can they tell us? Psychol Med 34(1): 3-8.

Rutten K, Lieben C, Smits L, Blokland A (2007). The PDE4 inhibitor rolipram reverses object memory impairment induced by acute tryptophan depletion in the rat. Psychopharmacology (Berl) 192(2): 275-282.

Scheepens A, Wassink G, Piersma MJ, Van de Berg WD, Blanco CE (2003). A delayed increase in hippocampal proliferation following global asphyxia in the neonatal rat. Brain Res Dev Brain Res 142(1): 67-76.

Shannon NJ, Gunnet JW, Moore KE (1986). A comparison of biochemical indices of 5hydroxytryptaminergic neuronal activity following electrical stimulation of the dorsal raphe nucleus. J Neurochem 47(3): 958-965.

Shimizu E, Hashimoto K, Okamura N, Koike K, Komatsu N, Kumakiri C, Nakazato M, Watanabe H, Shinoda N, Okada S, Iyo M (2003). Alterations of serum levels of brain-derived neurotrophic factor (BDNF) in depressed patients with or without antidepressants. Biol Psychiatry 54(1): 70-75.

Siuciak JA, Boylan C, Fritsche M, Altar CA, Lindsay RM (1996). BDNF increases monoaminergic activity in rat brain following intracerebroventricular or intraparenchymal administration. Brain Res 710(1-2): 11-20.

Siuciak JA, Clark MS, Rind HB, Whittemore SR, Russo AF (1998). BDNF induction of tryptophan hydroxylase mRNA levels in the rat brain. J Neurosci Res 52(2): 149-158.

Smith KA, Fairburn CG, Cowen PJ (1997). Relapse of depression after rapid depletion of tryptophan. Lancet 349(9056): 915-919.

Smith MA, Makino S, Kvetnansky R, Post RM (1995a). Effects of stress on neurotrophic factor expression in the rat brain. Ann N Y Acad Sci 771: 234-239.

Smith MA, Makino S, Kvetnansky R, Post RM (1995b). Stress and glucocorticoids affect the expression of brain-derived neurotrophic factor and neurotrophin-3 mRNAs in the hippocampus. J Neurosci 15(3 Pt 1): 1768-1777.

Stancampiano R, Melis F, Sarais L, Cocco S, Cugusi C, Fadda F (1997). Acute administration of a tryptophan-free amino acid mixture decreases 5 -HT release in rat hippocampus in vivo. Am J Physiol 272(3 Pt 2): R991-994.

Ueyama T, Kawai Y, Nemoto K, Sekimoto M, Tone S, Senba E (1997). Immobilization stress reduced the expression of neurotrophins and their receptors in the rat brain. Neurosci Res 28(2): 103-110.

Vaidya VA, Marek GJ, Aghajanian GK, Duman RS (1997). 5-HT2A receptor-mediated regulation of brain-derived neurotrophic factor mRNA in the hippocampus and the neocortex. J Neurosci 17(8): 2785-2795.

Van den Hove DL, Steinbusch HW, Scheepens A, Van de Berg WD, Kooiman LA, Boosten BJ, Prickaerts J, Blanco CE (2006). Prenatal stress and neonatal rat brain development. Neuroscience 137(1): 145-155.

van der Plasse G, Meerkerk DT, Lieben CK, Blokland A, Feenstra MG (2007). Lack of evidence for reduced prefrontal cortical serotonin and dopamine efflux after acute tryptophan depletion. Psychopharmacology (Berl) 195(3): 377-385.

van Donkelaar EL, Rutten K, Blokland A, Akkerman S, Steinbusch HW, Prickaerts J (2008). Phosphodiesterase 2 and 5 inhibition attenuates the object memory deficit induced by acute tryptophan depletion. Eur J Pharmacol 600(1-3): 98-104.

van Eijk HM, Rooyakkers DR, Deutz NE (1993). Rapid routine determination of amino acids in plasma by high-performance liquid chromatography with a 2-3 microns Spherisorb ODS II column. J Chromatogr 620(1): 143-148. 
Vollmayr B, Keck S, Henn FA, Schloss P (2000). Acute stress decreases serotonin transporter mRNA in the raphe pontis but not in other raphe nuclei of the rat. Neurosci Lett 290(2): 109-112.

Williams WA, Shoaf SE, Hommer D, Rawlings R, Linnoila M (1999). Effects of acute tryptophan depletion on plasma and cerebrospinal fluid tryptophan and 5-hydroxyindoleacetic acid in normal volunteers. J Neurochem 72(4): 1641-1647.

Young SN (1993). The use of diet and dietary components in the study of factors controlling affect in humans: a review. J Psychiatry Neurosci 18(5): 235-244. 
Chapter

\title{
Phosphodiesterase 2 and 5 inhibition attenuates the object memory deficit induced by acute tryptophan depletion
}

\author{
Eva L. van Donkelaar ${ }^{1}$, Kris Rutten ${ }^{1}$, Arjan Blokland ${ }^{2}$, \\ Sven Akkerman ${ }^{1}$, Harry W.M. Steinbusch ${ }^{1}$, Jos Prickaerts ${ }^{1}$ \\ ${ }^{1}$ Department of Neuroscience, Faculty of Health, Medicine and Life Sciences, School \\ for Mental Health and Neuroscience, Maastricht University, The Netherlands \\ ${ }^{2}$ Department of Neuropsychology and Psychopharmacology, Faculty of Psychology \\ and Neuroscience, Maastricht University, The Netherlands
}

European Journal of Pharmacology, 2008, 600: 98-104 



\begin{abstract}
The underlying mechanism of short-term memory improvement after inhibition of specific phosphodiesterases (PDEs) is still poorly understood. The present study aimed to reveal the ability of PDE5 and PDE2 inhibitors, that increase cyclic guanosine monophosphate (cGMP) and both cyclic adenosine monophosphate (cAMP) and cGMP, respectively, to reverse an object recognition deficit induced by acute tryptophan depletion. Acute tryptophan depletion is a pharmacological challenge tool to lower central serotonin (5-hydroxytryptamine; 5-HT) levels by depleting the availability of its dietary precursor tryptophan. Short-term object memory was tested in male Wistar rats by exposing them to the object recognition task. First, the effects of acute tryptophan depletion upon object recognition $2 \mathrm{~h}$ after administration of the nutritional mixture were established. Subsequently, acute tryptophan depletion was combined with the PDE5 inhibitor vardenafil $(1,3$ and $10 \mathrm{mg} / \mathrm{kg})$ or with the PDE2 inhibitor BAY 60-7550 (0.3, 1 and $3 \mathrm{mg} / \mathrm{kg}), 30 \mathrm{~min}$ prior to testing. Acute tryptophan depletion significantly lowered plasma tryptophan levels and impaired object recognition performance. Vardenafil (3 and $10 \mathrm{mg} / \mathrm{kg}$ ) and BAY $60-7550(3 \mathrm{mg} / \mathrm{kg}$ ) were able to attenuate the acute tryptophan depletion induced object recognition impairment. Thus, both PDE5 and PDE2 inhibition improved short-term object recognition performance after an acute tryptophan depletion induced deficit. The underlying mechanisms, however, remain poorly understood and further studies are needed to determine whether the present findings can be explained by a direct effect of enhanced cAMP and cGMP levels upon 5-HT activity, or even other neurotransmitter systems, and possibly an interaction with synthesis of nitric oxide or effects upon cerebral blood flow function.
\end{abstract}




\section{Introduction}

The second messenger molecules cyclic adenosine monophosphate (cAMP) and cyclic guanosine monophosphate (cGMP) play an important role in intracellular signalling and appear to be differentially involved in learning and memory processes (Bernabeu et al., 1996; Bernabeu et al., 1997; Prickaerts et al., 2002a; Rutten et al., 2007b). Both cAMP and cGMP are selectively hydrolyzed by phosphodiesterase (PDE) enzymes and inhibition of the PDEs appears to be a reliable method to improve memory processes by increasing the levels of either cAMP, cGMP or both (Blokland et al., 2006).

From the eleven subclasses of PDEs, enhanced memory performance has been demonstrated after inhibition of PDE2 (Boess et al., 2004; Rutten et al., 2007b), PDE4 (Rutten et al., 2006; Zhang et al., 2005) and PDE5 (Devan et al., 2006; Prickaerts et al., 2004). Only recently, pro-cognitive effects have also been observed after PDE9 inhibition (Van der Staay et al., in press) and inhibition of PDE10 is gaining increasing interest for the improvement of cognitive deficits, specifically those related to schizophrenia (Schmidt et al., 2008). The PDE inhibitors (PDE-Is) type 4 and 5 selectively increase central cAMP and cGMP, respectively. PDE2 breaks down both cAMP and cGMP, thus, inhibition leads to enhanced concentrations of both second messengers (Boess et al., 2004). Whereas several mechanisms may explain the cognitive enhancing properties of PDE inhibition for long-term memory, the underlying mechanism for shortterm memory improvement is still poorly understood (Boess et al., 2004; Devan et al., 2004; Rutten et al., 2007a).

Elevated levels of cAMP and cGMP enhance long term potentiation, which is assumed to be the underlying substrate of memory formation (Bliss and Collingridge, 1993), through activation of the cAMP/PKA/CREB (Impey et al., 1996) and cGMP/PKG/CREB pathway (Lu et al., 1999), respectively. This requires gene transcription or protein synthesis and provides an explanation for improvement of long-term memory processes after PDE inhibition (Blokland et al., 2006), not however for short-term memory enhancement. To date, shortterm memory improvement after PDE2 inhibition has only been demonstrated in mice exposed to a spontaneous alternation task, in which the PDE2-I 2(3,4-dimethoxybenzyl)-7-\{(1R)-1-[(1R)-1-hydroxyethyl $]$-4-phenylbutyl $\}$-5-methyl imidazo[5,1-f][1,2,4]triazin-4(3H)-one (BAY 60-7550) was able to fully reverse the working memory deficit induced by the $\mathrm{N}$-methyl-D-aspartate (NMDA) receptor blocker MK-801 (Boess et al., 2004). Improved object recognition performance was also found in rats after treatment with the less selective PDE5-I zaprinast, which reversed a memory deficit induced by the nitric oxide synthase (NOS) inhibitior 7-nitroindazole (Prickaerts et al., 1997). In a learning task for rats, NOS inhibition (NOS-I) with $\mathrm{N}$ (omega)-nitro-Larginine methyl ester (L-NAME) also induced impaired performance, which was attenuated after treatment with the highly selective PDE5-I sildenafil (Devan et al., 2006). Activation of presynaptic cGMP by elevated levels of nitric 
oxide (NO), which is a retrograde messenger (Murad et al., 1978), is shortlasting without the requirement of protein synthesis or gene transcription and might thus underlie short-term memory processes.

In general, a direct link has been proposed between the levels of cAMP and changes in neurotransmitter (acetylcholine, noradrenaline, serotonin and histamine) release and activity (Lourenco et al., 2006; Silvestre et al., 1999), which could underlie the improvement of short-term memory after inhibition of PDE4 (Rutten et al., 2006; Rutten et al., 2007a). Along similar lines, involvement of the serotonin (5-hydroxytryptophan; 5-HT) system was demonstrated in a recent study in which the highly selective PDE4-I rolipram was tested for object recognition performance in a serotonergic deficit model induced by acute tryptophan depletion (ATD; Rutten et al., 2007a). Rolipram reversed the ATD-induced short-term memory deficits, suggested to be due to an interaction with specific neurotransmitter systems.

An acute decrease of central 5-HT concentrations can be accomplished by applying the ATD method. ATD is a 5-HT challenge tool that manipulates the availability of the essential amino acid tryptophan (TRP), the dietary precursor of 5-HT (Bell et al., 2005; Neumeister et al., 2005; Young, 1993). By administering a TRP-free nutritional mixture, plasma TRP levels are depleted and less of the precursor is available for synthesis into 5-HT (Fadda et al., 2000; Fernstrom, 1983). The ATD method is widely used both clinically and preclinically as a tool to investigate the implication of the 5-HT system in affective disorders (Booij et al., 2003).

ATD-induced memory deficits have been observed in both healthy and serotonergic vulnerable human subjects (Booij et al., 2005; Evers et al., 2005; Jans et al., 2007b; Klaassen et al., 1999; Riedel et al., 1999; Riedel, 2004; Sambeth et al., 2007). Moreover, as mentioned above, Rutten et al. (2007a) demonstrated that ATD significantly reduces object recognition performance in Wistar rats both $1 \mathrm{~h}$ and $3 \mathrm{~h}$ after administration of the nutritional mixture. Similar short-term memory deficits induced by ATD in the rat object recognition task have also been demonstrated by others (Jans et al., 2007a; Lieben et al., 2004b; Lieben et al., 2005). Thus, the ATD method appears to be a reliable deficit model in which memory enhancing compounds, including PDE-Is, can be tested (Rutten et al., 2007a).

The aim of the present study was to further explore the link between PDE2 and PDE5 inhibition, the 5-HT system and changes in short-term memory performance. In addition to the short-term memory enhancing effects of the PDE4-I rolipram (Rutten et al., 2007a), we investigated the ability of the PDE5-I vardenafil and the PDE2-I BAY 60-7550 to reverse an ATD-induced impairment in object recognition performance. 


\section{Experimental procedures}

\section{Animals}

A total of 23 six-month-old male Wistar rats (Charles River, The Netherlands) were used, weighing between 450 and $500 \mathrm{~g}$ at the start of the experiments. On arrival, animals were housed individually in standard Makrolon TM (Type III) cages on sawdust bedding and maintained on a 12:12-hr light-dark schedule (lights on from 18:00 h - 06:00 h) in a temperature controlled $\left(21 \pm 2^{\circ} \mathrm{C}\right)$ and air-conditioned testing room. Animals were housed in the room where testing took place with background noise being provided by a radio playing softly at all times. Handling and treatment of the animals was not started until two weeks after arrival and food and tap water was freely available throughout this period.

All experimental procedures were approved by the local ethical Committee on Animal Experimentation of the Maastricht University and were in accordance with governmental guidelines.

\section{Drugs and chemicals}

The TRP-free gelatine hydrolysate $\left(\right.$ Solugel ${ }^{\circledR}$ ) was obtained from PB Gelatins (Tessenderlo Belgium). Glucodry 210 was obtained from the Amylumgroup (Koog aan de Zaan, The Netherlands). Potassium chloride (KCl) and calciumchloride-dihydrate $\left(\mathrm{CaCl}_{2} \cdot 2 \mathrm{H}_{2} \mathrm{O}\right)$ were purchased from Merck (Darmstadt, Germany). L-tryptophan was obtained from Sigma-Aldrich (Zwijndrecht, The Netherlands). Vardenafil and BAY 60-7550 were kindly donated by the Chemistry Department of Pharma Research, Bayer AG (Wuppertal, Germany).

\section{Treatments}

\section{Acute tryptophan depletion}

After adaptation to the new environment and the reversed light/dark cycle, animals were handled and habituated to the oral administrations by gavage, two weeks before the start of the experiments. Rats were food deprived overnight prior to each experimental day to avoid any TRP uptake from the standard animal chow. For acute reduction of plasma TRP levels, rats were administered a TRP-free protein-carbohydrate nutritional mixture (TRP- group). The gelatine-based protein nutritional mixture $\left(\right.$ Solugel $\left.{ }^{\circledR}\right)$ comprises the entire range of amino acids (see Table 1) in the form of peptides, as seen in normal food. The addition of carbohydrates activates the insulin response, thereby enhancing protein synthesis which results in an optimized reduction of plasma TRP levels (Lieben et al., 2004a; Markus et al., 1998). 
Table 1 Composition of the protein-carbohydrate nutritional mixture and determination of the amino acid content $(\mathrm{g} / 100 \mathrm{ml}$ water) of the gelatine based protein (Solugel $\mathbb{R}$ )

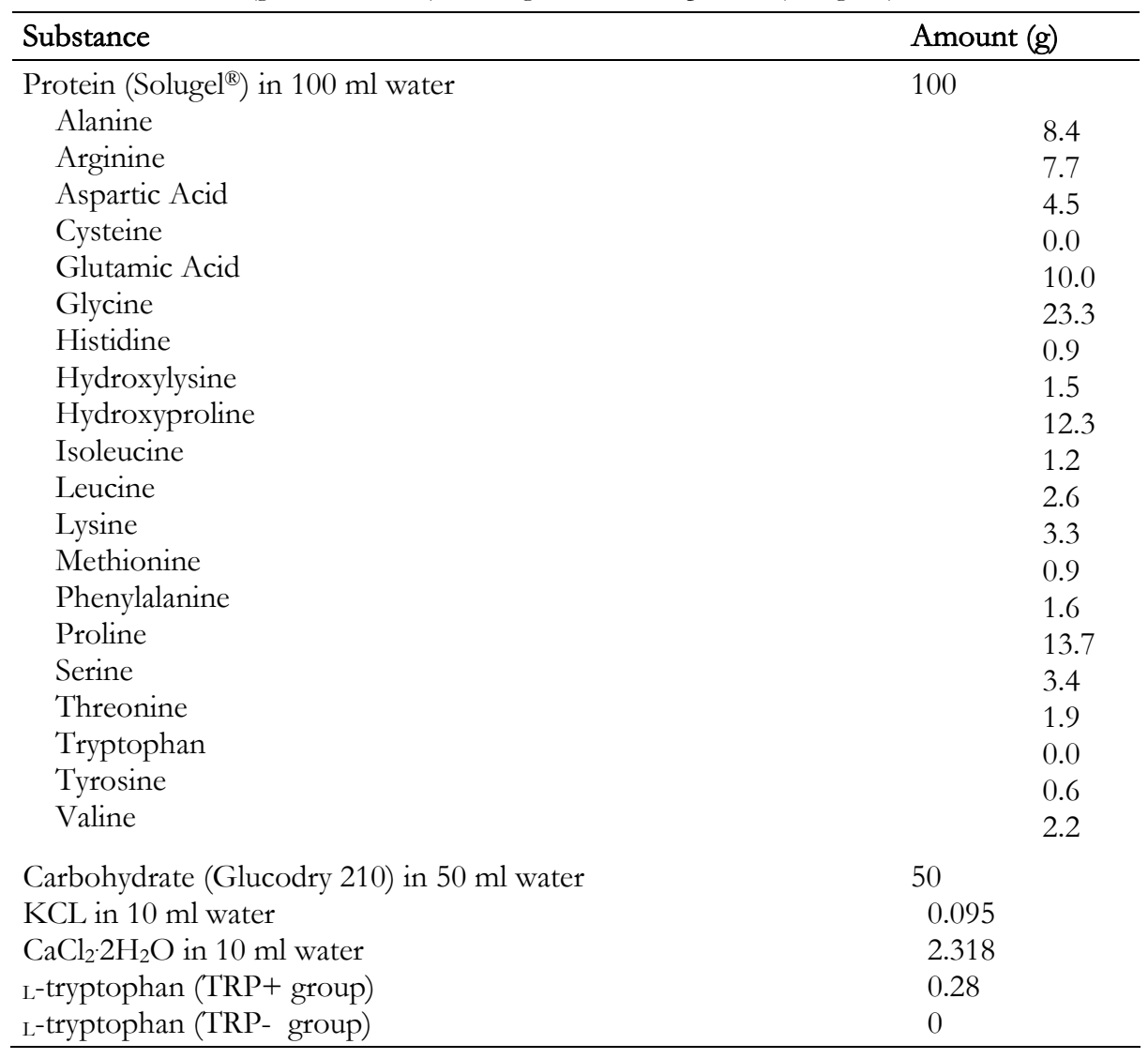

TRP availability for synthesis into 5-HT also depends on the intake of other large neutral amino acids (LNAAs; leucine, isoleucine, tyrosine, valine and phenylalaline), since they all compete for the same amino acid L-carrier transport system for crossing the blood brain barrier (Fernstrom and Wurtman, 1972; Fernstrom, 1983). Therefore, a decrease of the ratio TRP/ $\Sigma$ LNAAs has been used in the present study as the best predictor of reduced availability of central TRP (Fernstrom, 1979, 1983).

To the control mixture, L-TRP was added in an amount of $0.28 \%$ of the total protein content (TRP+ group). In addition, a saline group was included to control for any effects of the nutritional compounds in general. Nutritional mixtures were dissolved in distilled water and freshly prepared in the afternoon the day before and stored at $4{ }^{\circ} \mathrm{C}$ until the morning of the day of the experiment. Each experimental dose contained $4 \mathrm{~g} / \mathrm{kg}$ bodyweight of Solugel ${ }^{\circledR}$ 
and $2 \mathrm{~g} / \mathrm{kg}$ Glucodry and was administered once in a volume of $10 \mathrm{ml} / \mathrm{kg}$ between 8:30 and 12:30 h, 120 min prior to behavioural testing.

\section{Phosphodiesterase inhibitors}

Compounds were freshly prepared on the day of the experimental test trials and administered orally by gavage $30 \mathrm{~min}$ prior to the start of the first trial (T1) of the object recognition task and always in combination with ATD. Vardenafil was dissolved in 1\% tylose (methyl-cellulose) and tested in doses of 1, 3 and 10 $\mathrm{mg} / \mathrm{kg}$. BAY 60-7550 was suspended in 5\% Tween 80 and 1\% tylose and tested in doses $0.3,1$ and $3 \mathrm{mg} / \mathrm{kg}$. Both PDE-Is were administered in a volume of 2 $\mathrm{ml} / \mathrm{kg}$. Only the $1 \%$ tylose was used for the vehicle condition.

\section{Object recognition task}

The object recognition task was performed as described elsewhere (Prickaerts et al., 1997). The apparatus consisted of a circular arena, $83 \mathrm{~cm}$ in diameter. Half of the $40-\mathrm{cm}$ high wall was made of grey polyvinyl chloride, the other half of transparent polyvinyl chloride. During the test sessions only a light bulb was switched on with intensity (20 lux) equally spread over the different parts of the apparatus. Two objects were placed in symmetrical position, at about $10 \mathrm{~cm}$ away from the grey wall. Four different objects were used with each object being available in triplicate: (1) a cone consisting of a grey polyvinyl chloride base $(18 \mathrm{~cm}$ in diameter) with a collar on top made of brass (total height 16 $\mathrm{cm}$ ), (2) a standard transparent (dark-brown) glass bottle (diameter $10 \mathrm{~cm}$, height $22 \mathrm{~cm})$, (3) a massive metal cube $(10 \times 5 \times 7.5 \mathrm{~cm})$ with two holes (diameter $1.9 \mathrm{~cm})$ and (4) a massive aluminium cube with a tapering top $(13 \mathrm{x} 8$ $\mathrm{x} 8 \mathrm{~cm})$. The objects could not be displaced by the rats.

Adaptation and training procedures were similar as described by Rutten et al. (2007a). In the first week, the animals were handled daily and trained for the testing procedures. Training took place on two days, with one day in between and consisted of a 3 min exploration of the apparatus without objects. One week after the initial training sessions, animals were further adapted by exposing them to a $1 \mathrm{~h}$ and $24 \mathrm{~h}$ interval test session with objects, however, without any treatment applications. Subsequently, they were adapted to oral administration by gavage, once with saline $(10 \mathrm{ml} / \mathrm{kg})$ and once with the control mixture (TRP+; $10 \mathrm{ml} / \mathrm{kg}$ ). In the following weeks, the effect of ATD upon object recognition was tested and subsequently the drug compounds were tested in combination with ATD.

A test session included two trials of 3 min each with a $1 \mathrm{~h}$ interval. During T1 the apparatus contained two identical objects. A rat was always placed in the apparatus facing the wall in the centre of the transparent front segment. After the first exploration period, the rat was returned to its home cage. Subsequently, after a specific delay interval, the rat was put back into the 
apparatus for the second trial (T2). In T2 the apparatus contains two different objects, a familiar one (from T1) and a new one. The time spent exploring each object during T1 and T2 were recorded manually with a personal computer.

Exploration was defined as follows: directing the nose to the object at a distance of no more than $2 \mathrm{~cm}$ or touching the object with the nose. Sitting on the object or gnawing an object was not considered exploratory behaviour. To avoid the presence of olfactory trails and prevent any use of odour cues, the objects were thoroughly cleaned with water and $70 \%$ alcohol in between sessions. Moreover, each object was available in triplicate, with two objects being used in T1 and the third one for the recognition trial. This way, it was avoided that any of the two objects of T1 had to be used as the familiar object in T2. The order of the objects used per animal and per session was determined randomly and all combinations and locations of objects were used in a balanced manner to reduce potential biases due to preferences of particular locations or objects.

In the first experiment, the effects of a single dose of $10 \mathrm{ml} / \mathrm{kg}$ of either saline, TRP+ or TRP-, in combination with vehicle treatment, 30 min prior to testing, upon object memory was measured. In subsequent experiments, the ability of both vardenafil and BAY 60-7550 to reverse the ATD-induced impairment in object memory was tested. The same animals were used for all three experiments and all animals were exposed to each treatment condition in a randomized order. Three testing sessions were conducted per week with $48 \mathrm{~h}$ washout periods in between testing days.

\section{Blood samples}

At the end of the experiment, the ATD procedure was repeated, including 5 different treatment conditions, for blood sampling only. For determination of peripheral amino acid concentrations, a baseline blood sample was taken by means of saphenous vein puncture, 30 min prior to oral administration with either saline $(n=4), T R P+(n=4)$ or TRP- $(n=15)$. Thirty min before the animals were decapitated, the saline and TRP + treatment groups additionally received an oral administration with the vehicle solution. The TRP- group received either the vehicle solution $(n=5)$ or were treated with $3 \mathrm{mg} / \mathrm{kg}$ vardenafil $(n=5)$ or BAY 60-7550A ( $n=5)$. Dose was chosen based upon least effective dose in behavioural task and timing procedures for the specific treatments were in accordance with those applied for behavioural testing. A second blood sample was taken by collecting trunk blood at time of decapitation, i.e. 120 min after ATD and $30 \mathrm{~min}$ after treatment with the vehicle or the PDE-Is.

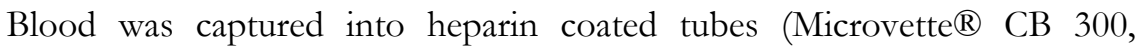
Sarstedt, Germany) which were kept on ice until centrifuged. After centrifugation for $10 \mathrm{~min}$ at 14,000 x g at $4{ }^{\circ} \mathrm{C}$ (Hettish EBA 12 centrifuge), plasma was transferred into cups which were immediately frozen in liquid nitrogen and stored at $-80{ }^{\circ} \mathrm{C}$ until further analysis. Free plasma amino acid 
concentrations were determined by means of fully automated high-performance liquid chromatography and expressed in $\mu \mathrm{mol}$ per liter.

\section{Statistical analyses}

\section{Biochemistry}

The mean concentrations of plasma amino acids were determined for each treatment condition and time-point separately and TRP/ $/$ LNAA ratios were calculated accordingly. Unexpectedly, a priori differences in baseline plasma ratios were found. Exclusion from analyses of extreme values (see below) might have contributed to the observed differences. Therefore, mean ratios (+ S.E.M.) are represented as percentage change form baseline levels.

Changes in the ratio TRP/ LLNAA over time were analyzed by means of twoway analysis of variance (ANOVA) with Treatment as between-subject factor and Time as within-subject variable. Subsequently, one-way ANOVA was performed to analyze differences between treatment conditions at the $120 \mathrm{~min}$ time-point. A post hoc Bonferroni test was used to further characterize the treatment effects.

Extreme values, as calculated with Dixon's Extremity test (Dixon, 1959), were excluded from statistical analysis. This occurred at baseline in the saline group ( $n=1)$ and in the TRP-/vehicle group $(n=1)$. At the 120 min time-point, extreme values were found in the TRP-/vehicle group $(n=1)$ and the TRP/BAY 60-7550 ( $\mathrm{n}=1)$ group.

\section{Behaviour}

Behavioural analyses were in line with previous similar studies (Rutten et al., 2007a). Basic parameters involved are depicted in Table 2 as adapted from Prickaerts et al. (1997).

Table 2 Basic parameters involved in the object recognition task

\begin{tabular}{ll}
\hline Exploration & Discrimination \\
\hline$e 1=a 1+a 2$ & $d 2=(b-a 3) / e 2$ \\
$e 2=a 3+b$ & \\
\hline
\end{tabular}

$e 1$ is the time spent in exploring both identical objects $(a 1+a 2)$ during the first trial. $e 2$ indicates the time spent exploring both the familiar (a3) and the new $(b)$ object in the second trial. $d 2$ indicates the relative difference in time spent between exploring the familiar and the new object, used as discrimination index.

Both $e 1$ and $e 2$ indicate the total exploration activity during T1 and T2, respectively, whereas $d 2$ is the relative difference in time spent between 
exploring the familiar and the new object corrected for total exploration activity (e2). All parameters were analysed with one-way ANOVA for each PDE-I treatment separately, complemented with a Dunnett post hoc test. Differences in exploration time between the doses of each PDE-I treatment were further characterized with a Bonferroni post hoc test.

All data are represented as mean + standard error of the mean (S.E.M.) and acceptable level of significance was generally set at $P<0.05$.

\section{Results}

\section{Plasma}

The percentage change of the ratio TRP/ $\Sigma$ LNAA in plasma is shown in Fig.1. ATD decreased the ratio over time $[F(1,14)=399.72 ; P<0.001]$ and this decrease over time appeared to be different for the treatment conditions $[F(4$, $14)=12.96 ; P<0.001]$. An overall treatment effect was found $[F(4,14)=8.026$; $P<0.01]$. Post hoc analysis showed that TRP+ treatment significantly differed from all TRP- treatments. No difference was found between the TRP-group treated with $3 \mathrm{mg} / \mathrm{kg}$ of vardenafil and the one treated with $3 \mathrm{mg} / \mathrm{kg}$ of BAY 60-7550. Two hours after ATD, decreased TRP/ ¿LNAA concentrations were observed in all TRP- treatment groups compared to both the saline and TRP+ treatment condition (see Fig.1). No differences were found between saline and TRP+ treatment. Also, no differences were found between the 3 different TRP- treatment conditions.
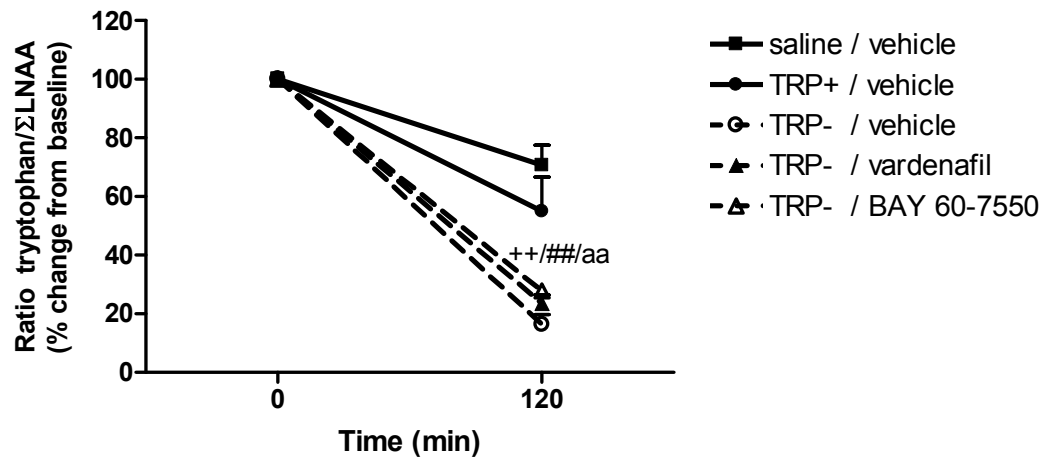

Figure 1 Percentage change of the ratio TRP/ $\mathrm{LNAA}$ compared to baseline levels (mean + S.E.M.) $120 \mathrm{~min}$ after treatment. Significant decreases were found after treatment with TRP/vehicle $\left(^{+}\right)$, TRP-/vardenafil (3 mg/kg) (\#) and TRP-/BAY 60-7550 (3 mg/kg) (a) compared to both TRP+/vehicle and saline/vehicle treatment. ${ }^{++/ \# \# / a a ~} P<0.01$. TRP-: tryptophan-free experimental mixture; TRP+: control mixture with tryptophan 


\section{Behaviour}

Table 3 shows the exploration times derived from all three behavioural experiments.

Table 3 Total exploration times (mean \pm S.E.M. in s) during the first $(e 1)$ and second $(e 2)$ trial of the object recognition task after ATD alone (Exp.1) or ATD in combination with different doses of vardenafil (Exp.2) or BAY 60-7550 (Exp.3)

\begin{tabular}{|c|c|c|c|c|c|}
\hline Experiment & Drug & ATD & Dose & $e 1$ & $e 2$ \\
\hline \multirow[t]{3}{*}{1} & vehicle & saline & & $199(17)$ & $323(28)$ \\
\hline & & TRP+ & & 203 (16) & $345(29)$ \\
\hline & & TRP - & & $223(17)$ & $303(18)$ \\
\hline \multirow[t]{3}{*}{2} & vardenafil & TRP - & $1 \mathrm{mg} / \mathrm{kg}$ & $227(17)$ & $264(20)$ \\
\hline & & TRP - & $3 \mathrm{mg} / \mathrm{kg}$ & 178 (14) & $295(18)$ \\
\hline & & TRP - & $10 \mathrm{mg} / \mathrm{kg}$ & $167(16)^{\mathrm{a}}$ & $270(22)$ \\
\hline \multirow[t]{3}{*}{3} & BAY 60-7550 & TRP - & $0.3 \mathrm{mg} / \mathrm{kg}$ & $290(29)$ & $286(19)$ \\
\hline & & TRP - & $1 \mathrm{mg} / \mathrm{kg}$ & $142(14)^{\mathrm{b}}$ & $244(15)$ \\
\hline & & TRP - & $3 \mathrm{mg} / \mathrm{kg}$ & $208(17)^{\mathrm{b}}$ & $294(27)$ \\
\hline
\end{tabular}

(a) indicates significantly different from $1 \mathrm{mg} / \mathrm{kg}$ (Exp. 2, $P<0.05$ ) (b) indicates significantly different from $0.3 \mathrm{mg} / \mathrm{kg}$ (Exp. 3, $P<0.05)$

\section{Experiment 1}

ATD did not have an effect upon the total exploration times during T1 or T2. The effect of ATD upon performance in the object recognition task is depicted in Fig. 2. An overall effect of ATD upon object recognition was found $[F(2,65)$ $=5.47 ; P<0.01]$. Post hoc analyses showed that after ATD, performance in de object recognition task significantly deteriorated compared to the animals that were treated with the TRP+ control mixture. No differences were found between the saline group and the TRP+ treatment condition.

\section{Experiment 2}

As is shown in Table 3, vardenafil treatment decreased the total exploration time in $\mathrm{T} 1[F(2,68)=4.10 ; P<0.05]$, however, not in $\mathrm{T} 2[F(2,68)=0.66$; n.s. $]$. Post hoc analysis showed that the total exploration time in T1 was significantly lower after the highest dose $(10 \mathrm{mg} / \mathrm{kg})$ of vardenafil compared to the lowest dose $(1 \mathrm{mg} / \mathrm{kg})$ of vardenafil. Fig. 3 shows the effects of ATD in combination with different doses of the PDE5-I vardenafil upon object recognition 
performance. A dose-dependent increase in object recognition performance was found after treatment with vardenafil $[F(3,91)=8.06 ; P<0.001]$. Post hoc analyses showed that both the $3 \mathrm{mg} / \mathrm{kg}$ dose and the $10 \mathrm{mg} / \mathrm{kg}$ dose of vardenafil reversed the effects of TRP- treatment.

\section{Experiment 3}

As is shown in Table 3, treatment with BAY 60-7550 decreased the total exploration time in T1 $[F(2,68)=12.49 ; P<0.001]$, however, not in T2 $[F(2$, $65)=1.64$; n.s.]. Post hoc analyses showed that total exploration time was significantly lower after treatment with both $1 \mathrm{mg} / \mathrm{kg}$ and the $3 \mathrm{mg} / \mathrm{kg}$ of BAY 60-7550, compared to the lowest dose $(0.3 \mathrm{mg} / \mathrm{kg})$. Fig. 4 shows the effects of ATD in combination with different doses of the PDE2-I BAY 60-7550. A dose-dependent increase in object recognition performance was found after treatment with BAY 60-7550 $[F(3,88)=4.64 ; P<0.01]$. Dunnett post hoc analyses revealed that the $3 \mathrm{mg} / \mathrm{kg}$ dose reversed the effects of TRP- treatment.

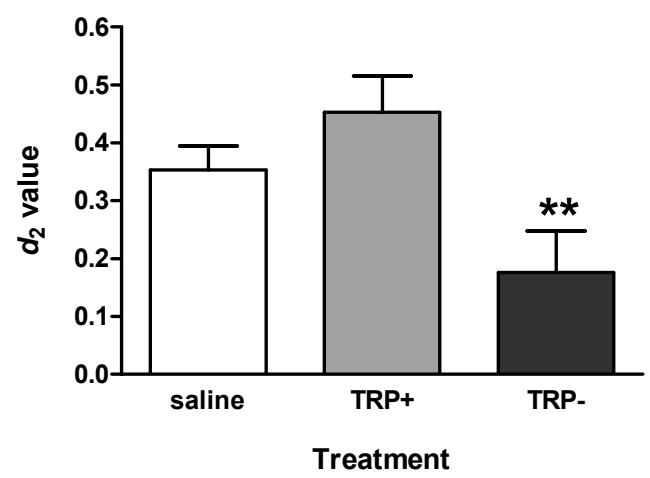

Figure 2 The effects (mean + S.E.M.) of a single dose of $10 \mathrm{ml} / \mathrm{kg}$ saline, TRP+ (control mixture with tryptophan) or TRP- (tryptophan-free experimental mixture) upon discrimination performance $(d 2)$ in the object recognition task, $2 \mathrm{~h}$ after administration and in combination with vehicle treatment $30 \mathrm{~min}$ prior to T1. ${ }^{* *} P<0.01$ indicates significantly different from TRP+ group. 


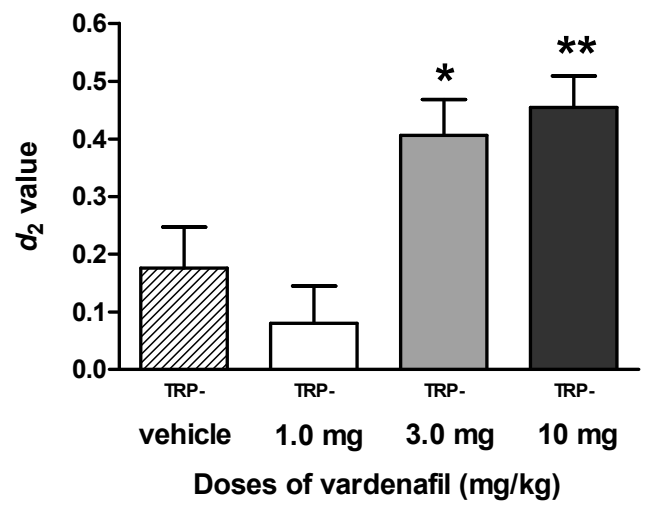

Figure 3 The effects (mean + S.E.M.) of vardenafil treatment upon ATD-induced deficits in the object recognition task. $\left(^{*}\right)$ indicates significant difference compared to the TRP-/vehicle treatment group $* P<0.05, * * P<0.01$

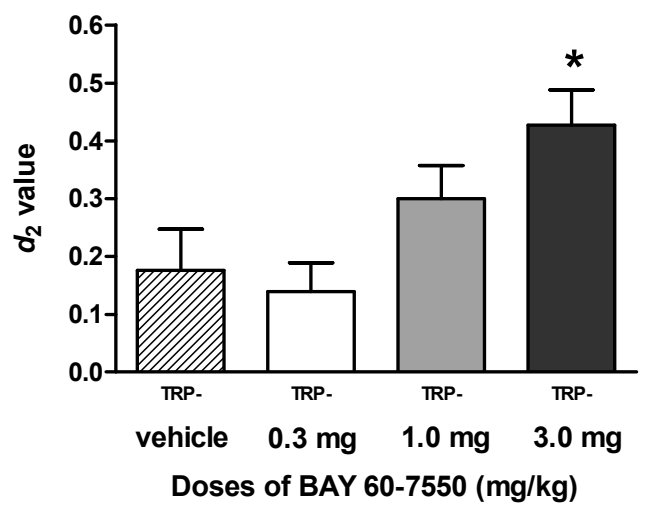

Figure 4 The effects (mean + S.E.M.) of BAY 60-7550 treatment upon ATD-induced deficits in the object recognition task. ${ }^{*} \mathrm{P}<0.05$ indicates significant difference compared to the TRP/vehicle treatment group. TRP-: tryptophan-free experimental mixture 


\section{Discussion}

In the present study, ATD was applied to impair short-term object recognition in rats and to be able to investigate the ability of PDE2 and PDE5 inhibitors to restore short-term memory deficits. ATD was established by administering a single dose of $10 \mathrm{ml} / \mathrm{kg}$ of a TRP-free protein-carbohydrate mixture, which resulted in a substantial decrease of the ratio TRP/ $/$ LNAA in plasma after $2 \mathrm{~h}$ and significantly impaired object recognition performance. Both the PDE5-I vardenafil (3 and $10 \mathrm{mg} / \mathrm{kg}$ ) and the PDE2-I BAY 60-7550 (3 mg/ $\mathrm{kg}$ ) were able to reverse the ATD-induced memory deficit when administered $30 \mathrm{~min}$ prior to testing, i.e. $90 \mathrm{~min}$ after ATD. ATD in combination with vardenafil and BAY 60-7550 treatment resulted in significant decreases of the ratio TRP/ LLNAA in plasma, without affecting the ratio differently compared to ATD alone. Therefore, the improvement in object recognition, as found in the present study, cannot be attributed to PDE-I induced normalization of the plasma TRP $/ \Sigma$ LNAA ratio.

Similar ATD-induced deficits in object memory performance have been found by others between 2 and $4 \mathrm{~h}$ after a double dose administration of the same nutritional mixture with reductions in the ratio TRP/ $/$ LNAA up to $60 \%$ and higher (Jans et al., 2007a; Lieben et al., 2004a). Corroborating earlier findings (Rutten et al., 2007a), our data suggest that a single administration of the same dose is already sufficient to reach a considerable reduction of the TRP/ LLNAA ratio and impair object memory within $2 \mathrm{~h}$. Taken together, these data indicate that a mean ratio TRP/ $\mathrm{LNNA}$ depletion of $50 \%$ and higher at $2 \mathrm{~h}$ compared to baseline levels, interferes with optimal cognitive performance in rats in this task.

Whereas ATD did not affect the exploration times, decreased total exploration was found in T1 after treatment with the highest dose of vardenafil $(10 \mathrm{mg} / \mathrm{kg})$ and after treatment with both 1 and $3 \mathrm{mg} / \mathrm{kg}$ of BAY $60-7550$. However, no differences were found in exploration times during T2. Moreover, effects upon exploration times appear to be independent of the effects on $d 2$ measurements (Sik et al., 2003). Of note, the effective doses of the PDE-Is in this study are relatively high as compared to PDE-I treatment alone. Most likely, side effects of PDE2 and PDE5 inhibition interfered with locomotor activity.

Treatment with vardenafil selectively inhibits enzymatic activity of PDE5, which leads to increased intracellular concentrations of the second messenger molecule cGMP as found in hippocampal slices (de Vente et al., 1996; Prickaerts et al., 2002b; van Staveren et al., 2001). BAY 60-7550 is a highly selective inhibitor of PDE2 and enhances the concentration of both cGMP and cAMP (Boess et al., 2004). The present study shows that both vardenafil and BAY 60-7550 are able to normalize an ATD-induced short-term memory deficit in the object recognition task. Attenuation of ATD-induced object 
memory impairment has also been observed after inhibition with the highly selective PDE4-I rolipram (Rutten et al., 2007a), which leads to increased levels of cAMP alone. The second messenger nucleotide cAMP appears to have a facilitatory effect upon the release of the neurotransmitters dopamine, norepinephrine and 5-HT (Schoffelmeer et al., 1985). Thus, inhibition of PDE4, which increases intracellular cAMP concentrations, might enhance 5-HT release and compensate for ATD-induced decreases in central 5-HT levels, thereby reversing the produced cognitive deficits. In addition, Lorenco et al. (2006) revealed that an increase in central 5-HT concentrations by means of selective 5-HT reuptake inhibitors corresponded to enhanced cAMP levels as measured by rolipram binding. Subsequently, it might be suggested that ATDinduced lowering of central 5-HT levels goes in line with decreased availability of cAMP, which might underlie the cognitive deficits found after ATD. Yet, the 5-HT enhancing properties of cAMP might only partly explain our results. The cognitive improving effects of rolipram might additionally be ascribed to its facility to enhance the cholinergic system, as it is known that rolipram also counteracts a cholinergic induced object memory impairment caused by scopolamine (Rutten et al., 2006).

Similar to our results, BAY 60-5770 was able to antagonize a short-term memory deficit induced by MK-801, which blocks NMDA receptor activity (Boess et al., 2004) and the PDE5-I zaprinast reversed an object memory deficit caused by NOS inhibition (Prickaerts et al., 1997). Likewise, the PDE5-I sildenafil was able to reverse an impairment induced by NOS inhibition in a rat learning task (Devan et al., 2006). However, the underlying mechanism of the enhancing effects of PDE-Is upon short-term memory processes remain rather unclear.

The cGMP/PKG pathway is implicated in the early phase of long-term potentiation (Arancio et al., 1995; Zhu et al., 2001), which might be linked to short-term memory (Izquierdo et al., 2002). The enhancing effects of PDE5 and PDE2 inhibition upon early phase long term potentiation can, besides directly increasing presynaptic cGMP levels, be mediated by activation of NOS post-synaptically (Domek-Lopacinska and Strosznajder, 2008). Since NO can freely diffuse back into the pre-synapse, it can increase cGMP levels by stimulating the synthesis of soluble guanylyl cyclase (sGC) (Murad et al., 1978). As the pre-synaptic mechanisms are short-lived and do not require gene transcription or protein synthesis, they might underlie the improvement of short-term object memory after PDE5 and PDE2 inhibition, as was found in the present study.

ATD might affect memory processes by affecting brain citrulline (CIT) concentrations, thereby decreasing NO synthesis (Prast and Philippu, 2001). It is known that arginine is catalysed into CIT and NO (Dawson and Dawson, 1996). Decreased concentrations of CIT after ATD have been observed by Lieben et al. (2004a). Recently, as was already referred to above, it was revealed that BAY 60-7550 and the PDE5-I zaprinast increased NOS activity in the rat 
hippocampus and striatum and improved object recognition performance after a $2 \mathrm{~h}$ interval (Domek-Lopacinska and Strosznajder, 2008). This effect of PDE inhibition upon NOS activity could also explain the present results by a direct effect upon 5-HT activity. Slight increases in NO concentrations most likely enhance 5-HT release whereas moderate increases rather decrease 5-HT release (Kaehler et al., 1999). The modulation of 5-HT release by NO might, therefore, depend on pre-existing NO concentrations and the effects might be differently regulated in distinct brain areas (Smith and Whitton, 2000). It remains to be demonstrated to what extent PDE5 and PDE2 inhibition leads to increases in NO concentrations.

The 5-HT neurotransmitter is a powerful vasoconstrictor, thus, alterations in endogenous 5-HT function may alter global or regional neuronal activity (Kelly et al., 1988). Consequently, ATD could result in an increase in local cerebral blood flow $(\mathrm{CBF})$ due to vasodilatation. Surprisingly, a decrease in local CBF after ATD has been reported in humans (Talbot and Cooper, 2006). Whether this is also the case for rats remains to be established, though, it could explain the ATD-induced object memory deficits. PDE-Is can increase central cAMP and cGMP concentrations, which are both known vasodilators (Dundore et al., 1993). Thus, PDE-Is could attenuate possible cerebrovascular effects of ATD. However, this needs to be elucidated in further studies.

In summary, both the PDE5 inhibitor vardenafil and the PDE2 inhibitor BAY 60-7550 are able to reverse an ATD-induced object memory deficit. The PDE2-I might have an effect via a direct modulation of 5-HT neurotransmission, although the exact underlying mechanism of action appears to be rather complex. Furthermore, inhibition of PDE5 and PDE2 might counteract the ATD-induced deficit possibly through increasing presynaptic cGMP levels by inhibiting its degradation. It remains to be investigated whether these PDE-Is can stimulate sGC directly through increased levels of NO, although the latter can also influence 5-HT levels itself. Further studies are needed to determine whether the present findings can be explained by a direct effect of enhanced cAMP and cGMP levels upon 5-HT activity or even other neurotransmitter systems, and possibly an interaction with synthesis of nitric oxide.

\section{References}

Arancio O, Kandel ER, Hawkins RD (1995). Activity-dependent long-term enhancement of transmitter release by presynaptic 3',5'-cyclic GMP in cultured hippocampal neurons. Nature 376(6535): 74-80.

Bell CJ, Hood SD, Nutt DJ (2005). Acute tryptophan depletion. Part II: clinical effects and implications. Aust N Z J Psychiatry 39(7): 565-574.

Bernabeu R, Schmitz P, Faillace MP, Izquierdo I, Medina JH (1996). Hippocampal cGMP and cAMP are differentially involved in memory processing of inhibitory avoidance learning. NeuroReport 7(2): 585-588. 
Bernabeu R, Schroder N, Quevedo J, Cammarota M, Izquierdo I, Medina JH (1997). Further evidence for the involvement of a hippocampal cGMP/cGMP-dependent protein kinase cascade in memory consolidation. NeuroReport 8(9-10): 2221-2224.

Bliss TV, Collingridge GL (1993). A synaptic model of memory: long-term potentiation in the hippocampus. Nature 361(6407): 31-39.

Blokland A, Schreiber R, Prickaerts J (2006). Improving memory: a role for phosphodiesterases. Curr Pharm Des 12(20): 2511-2523.

Boess FG, Hendrix M, van der Staay FJ, Erb C, Schreiber R, van Staveren W, de Vente J, Prickaerts J, Blokland A, Koenig G (2004). Inhibition of phosphodiesterase 2 increases neuronal cGMP, synaptic plasticity and memory performance. Neuropharmacology 47(7): 1081-1092.

Booij L, Van der Does AJ, Riedel WJ (2003). Monoamine depletion in psychiatric and healthy populations: review. Mol Psychiatry 8(12): 951-973.

Booij L, Van der Does AJ, Haffmans PM, Riedel WJ, Fekkes D, Blom MJ (2005). The effects of high-dose and low-dose tryptophan depletion on mood and cognitive functions of remitted depressed patients. J Psychopharmacol 19(3): 267-275.

Dawson VL, Dawson TM (1996). Nitric oxide actions in neurochemistry. Neurochem Int 29(2): 97-110.

de Vente J, Hopkins DA, Markerink-van Ittersum M, Steinbusch HW (1996). Effects of the 3',5'phosphodiesterase inhibitors isobutylmethylxanthine and zaprinast on NO-mediated cGMP accumulation in the hippocampus slice preparation: an immunocytochemical study. J Chem Neuroanat 10(3-4): 241-248.

Devan BD, Sierra-Mercado D, Jr., Jimenez M, Bowker JL, Duffy KB, Spangler EL, Ingram DK (2004). Phosphodiesterase inhibition by sildenafil citrate attenuates the learning impairment induced by blockade of cholinergic muscarinic receptors in rats. Pharmacology, biochemistry, and behavior 79(4): 691-699.

Devan BD, Bowker JL, Duffy KB, Bharati IS, Jimenez M, Sierra-Mercado D, Jr., Nelson CM, Spangler EL, Ingram DK (2006). Phosphodiesterase inhibition by sildenafil citrate attenuates a maze learning impairment in rats induced by nitric oxide synthase inhibition. Psychopharmacology 183(4): 439-445.

Dixon WJ (1959). Analysis of extreme values. Ann Math Stat 21: 488-506.

Domek-Lopacinska K, Strosznajder JB (2008). The effect of selective inhibition of cyclic GMP hydrolyzing phosphodiesterases 2 and 5 on learning and memory processes and nitric oxide synthase activity in brain during aging. Brain Res 1216: 68-77.

Dundore RL, Clas DM, Wheeler LT, Habeeb PG, Bode DC, Buchholz RA, Silver PJ, Pagani ED (1993). Zaprinast increases cyclic GMP levels in plasma and in aortic tissue of rats. Eur J Pharmacol 249(3): 293-297.

Evers EA, Tillie DE, van der Veen FM, Lieben CK, Jolles J, Deutz NE, Schmitt JA (2005). Effects of a novel method of acute tryptophan depletion on plasma tryptophan and cognitive performance in healthy volunteers. Psychopharmacology 178(1): 92-99.

Fadda F, Cocco S, Stancampiano R (2000). A physiological method to selectively decrease brain serotonin release. Brain Res Brain Res Protoc 5(3): 219-222.

Fernstrom JD, Wurtman RJ (1972). Brain serotonin content: physiological regulation by plasma neutral amino acids. Science 178(59): 414-416.

Fernstrom JD (1979). Diet-induced changes in plasma amino acid pattern: effects on the brain uptake of large neutral amino acids, and on brain serotonin synthesis. J Neural Transm Suppl(15): 55-67.

Fernstrom JD (1983). Role of precursor availability in control of monoamine biosynthesis in brain. Physiol Rev 63(2): 484-546.

Impey S, Mark M, Villacres EC, Poser S, Chavkin C, Storm DR (1996). Induction of CREmediated gene expression by stimuli that generate long-lasting LTP in area CA1 of the hippocampus. Neuron 16(5): 973-982. 
Izquierdo LA, Barros DM, Vianna MR, Coitinho A, deDavid e Silva T, Choi H, Moletta B, Medina JH, Izquierdo I (2002). Molecular pharmacological dissection of short- and longterm memory. Cell Mol Neurobiol 22(3): 269-287.

Jans LA, Lieben CK, Blokland A (2007a). Influence of sex and estrous cycle on the effects of acute tryptophan depletion induced by a gelatin-based mixture in adult Wistar rats. Neuroscience 147(2): 304-317.

Jans LA, Riedel WJ, Markus CR, Blokland A (2007b). Serotonergic vulnerability and depression: assumptions, experimental evidence and implications. Mol Psychiatry 12(6): 522-543.

Kaehler ST, Singewald N, Sinner C, Philippu A (1999). Nitric oxide modulates the release of serotonin in the rat hypothalamus. Brain Res 835(2): 346-349.

Kelly PA, Davis CJ, Goodwin GM (1988). Differential patterns of local cerebral glucose utilization in response to 5-hydroxytryptamine agonists. Neuroscience 25(3): 907-915.

Klaassen T, Riedel WJ, Deutz NE, van Someren A, van Praag HM (1999). Specificity of the tryptophan depletion method. Psychopharmacology 141(3): 279-286.

Lieben CK, Blokland A, Westerink B, Deutz NE (2004a). Acute tryptophan and serotonin depletion using an optimized tryptophan-free protein-carbohydrate mixture in the adult rat. Neurochem Int 44(1): 9-16.

Lieben CK, van Oorsouw K, Deutz NE, Blokland A (2004b). Acute tryptophan depletion induced by a gelatin-based mixture impairs object memory but not affective behavior and spatial learning in the rat. Behav Brain Res 151(1-2): 53-64.

Lieben CK, Blokland A, Sik A, Sung E, van Nieuwenhuizen P, Schreiber R (2005). The selective 5-HT6 receptor antagonist Ro4368554 restores memory performance in cholinergic and serotonergic models of memory deficiency in the rat. Neuropsychopharmacology 30(12): 2169-2179.

Lourenco CM, Kenk M, Beanlands RS, DaSilva JN (2006). Increasing synaptic noradrenaline, serotonin and histamine enhances in vivo binding of phosphodiesterase-4 inhibitor (R)[11C]rolipram in rat brain, lung and heart. Life Sci 79(4): 356-364.

Lu YF, Kandel ER, Hawkins RD (1999). Nitric oxide signaling contributes to late-phase LTP and CREB phosphorylation in the hippocampus. J Neurosci 19(23): 10250-10261.

Markus CR, Panhuysen G, Tuiten A, Koppeschaar H, Fekkes D, Peters ML (1998). Does carbohydrate-rich, protein-poor food prevent a deterioration of mood and cognitive performance of stress-prone subjects when subjected to a stressful task? Appetite 31(1): 49-65.

Murad F, Mittal CK, Arnold WP, Katsuki S, Kimura H (1978). Guanylate cyclase: activation by azide, nitro compounds, nitric oxide, and hydroxyl radical and inhibition by hemoglobin and myoglobin. Adv Cyclic Nucleotide Res 9: 145-158.

Neumeister A, Yuan P, Young TA, Bonne O, Luckenbaugh DA, Charney DS, Manji H (2005). Effects of tryptophan depletion on serum levels of brain-derived neurotrophic factor in unmedicated patients with remitted depression and healthy subjects. Am J Psychiatry 162(4): 805-807.

Prast H, Philippu A (2001). Nitric oxide as modulator of neuronal function. Prog Neurobiol 64(1): 51-68.

Prickaerts J, Steinbusch HW, Smits JF, de Vente J (1997). Possible role of nitric oxide-cyclic GMP pathway in object recognition memory: effects of 7-nitroindazole and zaprinast. Eur J Pharmacol 337(2-3): 125-136.

Prickaerts J, de Vente J, Honig W, Steinbusch HW, Blokland A (2002a). cGMP, but not cAMP, in rat hippocampus is involved in early stages of object memory consolidation. Eur J Pharmacol 436(1-2): 83-87.

Prickaerts J, van Staveren WC, Sik A, Markerink-van Ittersum M, Niewohner U, van der Staay FJ, Blokland A, de Vente J (2002b). Effects of two selective phosphodiesterase type 5 inhibitors, sildenafil and vardenafil, on object recognition memory and hippocampal cyclic GMP levels in the rat. Neuroscience 113(2): 351-361.

Prickaerts J, Sik A, van Staveren WC, Koopmans G, Steinbusch HW, van der Staay FJ, de Vente J, Blokland A (2004). Phosphodiesterase type 5 inhibition improves early memory consolidation of object information. Neurochem Int 45(6): 915-928. 
Riedel WJ, Klaassen T, Deutz NE, van Someren A, van Praag HM (1999). Tryptophan depletion in normal volunteers produces selective impairment in memory consolidation. Psychopharmacology (Berl) 141(4): 362-369.

Riedel WJ (2004). Cognitive changes after acute tryptophan depletion: what can they tell us? Psychol Med 34(1): 3-8.

Rutten K, Prickaerts J, Blokland A (2006). Rolipram reverses scopolamine-induced and timedependent memory deficits in object recognition by different mechanisms of action. Neurobiol Learn Mem 85(2): 132-138.

Rutten K, Lieben C, Smits L, Blokland A (2007a). The PDE4 inhibitor rolipram reverses object memory impairment induced by acute tryptophan depletion in the rat. Psychopharmacology (Berl) 192(2): 275-282.

Rutten K, Prickaerts J, Hendrix M, van der Staay FJ, Sik A, Blokland A (2007b). Time-dependent involvement of cAMP and cGMP in consolidation of object memory: studies using selective phosphodiesterase type 2, 4 and 5 inhibitors. Eur J Pharmacol 558(1-3): 107-112.

Sambeth A, Blokland A, Harmer CJ, Kilkens TO, Nathan PJ, Porter RJ, Schmitt JA, Scholtissen B, Sobczak S, Young AH, Riedel WJ (2007). Sex differences in the effect of acute tryptophan depletion on declarative episodic memory: a pooled analysis of nine studies. Neurosci Biobehav Rev 31(4): 516-529.

Schmidt CJ, Chapin DS, Cianfrogna J, Corman ML, Hajos M, Harms JF, Hoffman WE, Lebel LA, McCarthy SA, Nelson FR, Proulx-LaFrance C, Majchrzak MJ, Ramirez AD, Schmidt K, Seymour PA, Siuciak JA, Tingley FD, 3rd, Williams RD, Verhoest PR, Menniti FS (2008). Preclinical characterization of selective phosphodiesterase 10A inhibitors: a new therapeutic approach to the treatment of schizophrenia. J Pharmacol Exp Ther 325(2): 681-690.

Schoffelmeer AN, Wardeh G, Mulder AH (1985). Cyclic AMP facilitates the electrically evoked release of radiolabelled noradrenaline, dopamine and 5-hydroxytryptamine from rat brain slices. Naunyn Schmiedebergs Arch Pharmacol 330(1): 74-76.

Sik A, van Nieuwehuyzen P, Prickaerts J, Blokland A (2003). Performance of different mouse strains in an object recognition task. Behav Brain Res 147(1-2): 49-54.

Silvestre JS, Fernandez AG, Palacios JM (1999). Preliminary evidence for an involvement of the cholinergic system in the sedative effects of rolipram in rats. Pharmacology, biochemistry, and behavior 64(1): $1-5$.

Smith JC, Whitton PS (2000). Nitric oxide modulates N-methyl-D-aspartate-evoked serotonin release in the raphe nuclei and frontal cortex of the freely moving rat. Neurosci Lett 291(1): $5-8$.

Talbot PS, Cooper SJ (2006). Anterior cingulate and subgenual prefrontal blood flow changes following tryptophan depletion in healthy males. Neuropsychopharmacology 31(8): 17571767.

Van der Staay FJ, Rutten K, Barfacker L, De Vry J, Erb C, Heckroth H, Karthaus D, Tersteegen A, Van Kampen M, Blokland A, Prickaerts J, Reymann KG, Schroder UH, Hendrix M (in press). The novel selective PDE9 inhibitor BAY 73-6691 improves learning and memory in rodents. Neuropharmacology.

van Staveren WC, Markerink-van Ittersum M, Steinbusch HW, de Vente J (2001). The effects of phosphodiesterase inhibition on cyclic GMP and cyclic AMP accumulation in the hippocampus of the rat. Brain Res 888(2): 275-286.

Young SN (1993). The use of diet and dietary components in the study of factors controlling affect in humans: a review. J Psychiatry Neurosci 18(5): 235-244.

Zhang HT, Huang Y, Suvarna NU, Deng C, Crissman AM, Hopper AT, De Vivo M, Rose GM, O'Donnell JM (2005). Effects of the novel PDE4 inhibitors MEM1018 and MEM1091 on memory in the radial-arm maze and inhibitory avoidance tests in rats. Psychopharmacology (Berl) 179(3): 613-619.

Zhu J, Mix E, Winblad B (2001). The antidepressant and antiinflammatory effects of rolipram in the central nervous system. CNS Drug Rev 7(4): 387-398. 


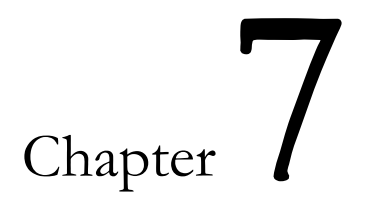

\section{General Discussion}

Eva L. van Donkelaar 

The implication of the serotonin (5-hydroxytryptamine; 5-HT) system and serotonergic vulnerability in the onset of depressive symptomatology is being extensively investigated with tools that challenge 5-HT neurotransmission. Most widely used, clinically and pre-clinically, is the method of acute tryptophan depletion (ATD), which manipulates the availability of the essential amino acid TRP, the dietary 5-HT precursor. By administering a TRP-free diet, plasma TRP levels are depleted, thereby decreasing the availability of TRP in the brain and its synthesis into 5-HT. ATD-induced behavioural changes are, therefore, generally attributed to the presumed decrease in 5-HT release and subsequent blunted neurotransmission. However, direct evidence that ATD decreases extracellular 5-HT concentrations is lacking and it remains unclear to what extent changes in 5-HT neuronal activity contribute to ATD-induced neurochemical and behavioural alterations. Moreover, in rats, degrees of peripheral and central TRP reduction, as well as brain 5-HT concentrations and behavioural effects have not been consistently reported. To improve the interpretation of clinical ATD-induced symptoms and provide new insights into the implication of the 5-HT system in depressive disorders, the present thesis covers studies exploring potential alternative mechanisms by which ATD exerts its neurophysiological and behavioural effects in rodents.

ATD was induced by administering a gelatin-based (i.e., TRP-free) proteincarbohydrate nutritional mixture $\left(\right.$ Soluge $^{\circledR}$ ) which is well established in rats (Lieben et al., 2004a) and most suitable for comparing the implication of 5-HT in neurophysiological functions across species (Blokland et al., 2004). In search of genetic 5-HT-related vulnerability factors implicated in the onset of depressive disorders, it was initially intended to establish the rat model of ATD in mice with the aim to further apply the 5-HT challenge tool in genetic mouse models of depression (chapter 2). Several explorative studies in Swiss and C57BL/6 mice revealed that in both strains the extent of peripheral TRP depletion highly depended upon the specificity of the dosing volume and the time-frame of the multiple oral administrations. Moreover, extreme differences in ATD effects were found between strains and none of the profound peripheral TRP depletions paralleled central TRP or 5-HT changes, nor induced any anxiety or depressive-like behaviour. Yet, interfering ATD application-related stress effects were found to confound the ATD-induced effects upon the ratio of TRP to the sum of other large neutral amino acids (TRP/ $\Sigma$ LNAA) in plasma. To gain more insight into the potential mechanisms underlying the ATD-induced neurochemical and behavioural effects in general, explorative experiments were continued in the established rat model. Whereas significant reductions of peripheral TRP levels and the ratio TRP/ $L$ LNAA were observed, no ATD-induced measurable effects upon TRP and 5-HT levels in the brain could be revealed (Table1). It might be argued that the extent of the peripheral depletion was not sufficient. However, despite the lack of a clear central effect, ATD significantly reduced local cerebral blood flow (CBF) 
in subregions of the hippocampus (chapter 3) and potentiated cerebrovascular dysfunction induced by repeated 3,4-methylenedioxymethamphetamine (MDMA; 'ecstasy') treatment (chapter 4). Moreover, moderate reduction of precursor availability in plasma significantly impaired object memory performance which was acutely reversed by phosphodiesterase (PDE) inhibitors that increase cyclic adenosine monophosphate (cAMP) and/or cyclic guanosine monophosphate (cAMP) (chapter 6). Furthermore, ATD did not directly affect peripheral or central brain-derived neurotrophic factor (BDNF) protein levels, but a positive correlation was found between TRP and BDNF in the brain (chapter 5). Interestingly, in line with the confounding ATD application-related stress effects in mice, BDNF was significantly lower in the prefrontal cortex of all animals that underwent ATD-related application procedures compared to a group that was not exposed to any experimental stress, such as food deprivation or oral administration by gavage.

Taken together, neurophysiological and behavioural changes were observed after significant ATD-induced reductions of precursor availability in plasma, yet in the absence of central 5-HT reductions. These results support the notion that while ATD-induced cognitive dysfunction and affective behavioural alterations in both animals and human subjects can be directly attributed to a lowering of peripheral TRP concentrations, the exact underlying mechanism might go beyond a straightforward alteration in the 5-HT system itself.

Table 1 Peripheral and central effect of acute tryptophan depletion in adult male Wistar rats as described in the studies of this thesis

\begin{tabular}{|c|c|c|c|c|c|c|c|}
\hline & \multirow[b]{3}{*}{ Study } & \multirow[b]{3}{*}{ Time } & \multirow{3}{*}{$\begin{array}{l}\text { Plasma } \\
\begin{array}{l}\% \text { change from } \\
\text { baseline }\end{array} \\
\text { TRP } / \Sigma \text { LNAA }\end{array}$} & \multicolumn{4}{|l|}{ Brain } \\
\hline & & & & \multicolumn{4}{|c|}{$\%$ difference from TRP+ group } \\
\hline Chapter & & & & TRP & 5-HT & 5-HIAA & 5-HIAA/5-HT \\
\hline 3 & $\begin{array}{l}\text { ATD/ } \\
\text { Cerebrovascular } \\
\text { dynamics }\end{array}$ & $\mathrm{T} 4$ & $-40 \%$ & - & - & - & - \\
\hline 4 & ATD / MDMA & $\mathrm{T} 4$ & $\begin{array}{l}-37 \% \text { (MDMA) } \\
-40 \% \text { (control) }\end{array}$ & - & - & - & - \\
\hline 5 & ATD / BDNF & $\mathrm{T} 4$ & $-56 \%$ & - & - & $\begin{array}{l}-33 \% \\
\text { (hippo) }\end{array}$ & $\begin{array}{l}-28 \% \\
\text { (hippo) }\end{array}$ \\
\hline 6 & ATD / PDE-Is & T2 & $-73 \%$ & n.a. & n.a. & n.a. & n.a. \\
\hline
\end{tabular}

ATD: acute tryptophan depletion (TRP-treatment); BDNF: brain-derived neurotrophic factor; hippo: hippocampus; MDMA: 3,4-methylenedioxymethamphetamine ('ecstasy'); PDE-Is: phosphodiesterase inhibitors; $\mathrm{T}$ : time in $\mathrm{h}$ after first administration; TRP: tryptophan; TRP/ $L$ LNAA: the ratio TRP to the sum of other large neutral amino acids; 5-HT: 5-hydroxytryptamine, serotonin; 5-HIAA: 5-hydroxyindoleacetic acid; (-) no change 


\section{Methodological considerations}

\section{Measuring central effects of acute tryptophan depletion}

\section{The ratio TRP/ $\Sigma L N A A$ in plasma}

ATD-induced reductions in central 5-HT availability have been extensively reported in both human subjects and animals. Yet, as outlined in the introduction of this thesis, most parameters applied to indicate reduced 5-HT synthesis or release appear to merely estimate decreases in 5-HT metabolism and neuronal activity, respectively, thereby remaining rather speculative. In general, the ratio TRP/ $\Sigma$ LNAA in plasma is used to estimate the amount of TRP available in the brain for synthesis into 5-HT, whereas in practise it provides only a rough estimation of the amount of TRP in the blood that will eventually be available to cross the blood-brain barrier (BBB). In most cases, including the studies in the present thesis, total peripheral TRP levels (free plus albumin-bound) are used for calculating the ratio to the sum of the other LNAAs. Although only the relatively small fraction of free TRP eventually crosses the BBB, TRP can easily dissociate from albumin near the BBB, thereby increasing the TRP-free pool and subsequent uptake into the brain. However, the direct effect of physiological factors such as hormones, exercise or mild stressors upon the rate of dissociation of TRP from albumin can only be taken into account if both total and free TRP levels are actually measured (Badawy, 2009), which is normally not done. Thus, based upon total peripheral TRP levels alone, the ratio TRP/ $L \mathrm{LNAA}$ in plasma might result in a distorted estimation of the rate of influx of TRP into the brain.

Moreover, other large neutral amino acids such as methionine (MET) and threonine (THR) are generally not included in the ratio as active competitors of plasma TRP. However, evidence exists that, together with the branched-chain amino acids (valine, leucine, isoleucine) and the aromatic amino acids (TRP, tyrosine, phenylalaline), MET and THR share the same L-amino acid transport carrier at the BBB (Oldendorf and Szabo, 1976; Pardridge, 1998; Smith, 2000). Thus, calculating the ratio TRP/ LLNAA without taking into account all competitive amino acids is most likely to result in an overestimation of brain TRP influx and subsequent dissociation from brain TRP levels.

\section{The ratio 5-HLAA/5-HT in the brain}

In contrast to human subjects, animal models provide the possibility to directly measure changes in TRP, 5-HT and 5-HIAA concentration in specific areas of the brain. Only a few rat studies of similar experimental design as that used in the studies of this thesis, including the same nutritional mixture, have reported ATD-induced decreases in central TRP and/or 5-HT (Jans et al., 2008; Jans et 
al., 2007; Lieben et al., 2004a; Olivier et al., 2008), mainly in the hippocampus. However, besides the fact that these central changes generally fail to parallel the acute changes in peripheral TRP levels, the extent of depletion seems highly variable between studies. In the studies of the present thesis, no significant changes in central TRP levels or 5-HT concentrations were observed, despite the considerable decrease in the ratio TRP/ $\mathrm{LNAA}$ in plasma. Although this might provide evidence for the fact that ATD does not considerably interfere with 5-HT synthesis in the brain, the ratio 5-HIAA/5-HT has been reported to better reflect changes in 5-HT synthesis and metabolism in general (Shannon et al., 1986). Together with brain 5-HIAA concentrations, the ratio 5-HIAA/5-HT is normally used to calculate the 5-HT turnover rate and estimate changes in 5-HT release, reflecting neuronal activity. However, this seems to apply only under normal physiological conditions when the rate of 5-HT synthesis remains constant. An increase in 5-HIAA levels would then increase the ratio 5-HIAA/5-HT and allows proper estimation of an increase in 5-HT neuronal activity, as more 5-HT has presumably been released. Conversely, a decrease in 5-HIAA levels under a constant rate of 5-HT synthesis would decrease the ratio 5-HIAA/5-HT. Yet, ATD is applied to induce a decrease in 5-HT synthesis. As a decrease in intracellular 5-HT availability most likely results in a reduction of the amount of 5-HT available for release, less extracellular 5-HT will then be available to be catabolyzed into 5-HIAA. Thus, ATD is likely to decrease both 5-HT and 5-HIAA, thereby maintaining the ratio 5-HIAA/5-HT constant. In line with this, no changes in the ratio 5-HIAA/5-HT were found $3 \mathrm{hr}$ after ATD, whereas both 5-HT $(-23 \%)$ and 5-HIAA $(-39 \%)$ levels appeared to be significantly decreased in rat hippocampus (Brown et al., 1998). A decrease in this ratio after ATD would only occur if significantly less 5-HIAA is produced compared to the already reduced amount of 5-HT synthesised.

The conversion of TRP into the 5-HTP intermediate by the TPH2 isoform is the first and rate-limiting step in the biosynthesis of 5-HT. In the brain, this enzyme is only $50 \%$ saturated and therefore, the rate at which 5 -HT is synthesised is limited only by substrate (i.e., TRP) availability (Boadle-Biber, 1993). However, it seems difficult to directly attribute an ATD-induced decrease in the ratio 5-HIAA/5-HT to a reduction in TRP availability. Brain measurements are generally taken at only one specific time point which impedes a comparison of the central parameters to baseline values. In one of the studies (chapter 5), a decrease in 5-HIAA levels and in the ratio 5-HIAA/5-HT was observed in the hippocampus. Such changes are normally interpreted as evidence for an ATD-induced reduction in 5-HT metabolism. However, 5-HT levels were not changed compared to TRP + treatment and information upon baseline 5-HT values is lacking. Similar reductions of the ratio 5-HIAA/5-HT have also been observed by others after ATD in rats, but between the studies concomitant changes in 5-HIAA or 5-HT in specific brain areas do not seem to be in accordance with each other (Ardis et al., 2009; Cahir 
et al., 2008; Jans et al., 2008). In general, it appears that ATD-induced absolute changes in central 5-HT or 5-HIAA concentrations and potential underlying mechanisms require further examination.

A decrease in the ratio 5-HIAA/5-HT after ATD seems more likely to be caused by factors influencing the catabolism of 5-HT, such as fluctuations in the activity of the monoamine oxidase (MAO) enzyme (Fernstrom and Hirsch, 1977). An acute decrease in MAO activity would reduce the absolute amount of 5-HIAA produced, thereby producing a relative increase in 5-HT levels. This would eventually lower the ratio 5-HIAA/5-HT independent of a reduction in precursor availability per se or the subsequent decrease in 5-HT synthesis and as such does not reflect a decrease in 5-HT release. Although an acute change in MAO activity could imply a compensatory mechanism activated upon the acute decrease in peripheral TRP levels, such a mechanism has not been described previously and thus remains to be further elucidated.

Other rat studies of similar experimental design have directly reported ATD-induced decreased 5-HT levels after administration of the same TRP-free nutritional mixture (Jans et al., 2008; Lieben et al., 2004a; Olivier et al., 2008). This is generally thought to reflect an ATD-induced decrease in 5-HT synthesis. However, as mentioned previously, the decrease in 5-HT after TRPtreatment at one specific time point is normally only compared to the control TRP+ treatment group and not to its own baseline concentrations. Thus, without these baseline values prior to treatment, the extent to which the significant difference in 5-HT levels between the experimental (TRP-) and control (TRP+) treatment group actually imply a reduction in 5-HT release after ATD remains unclear. In addition, actual reductions of basal 5-HT release after ATD have only been reported in combination with 5-HT reuptake inhibitors (Bel and Artigas, 1996; Fadda et al., 2000; Stancampiano et al., 1997) not, however, without an initial increase of extracellular 5-HT levels (Trulson, 1985; van der Plasse et al., 2007). It might be suggested that in the absence of de novo synthesis, 5-HT function can largely be maintained from transmitter being recycled into the presynaptic cell from the synaptic cleft. A possible decrease in 5-HT and 5-HIAA might then reflect a decrease in the storage pool of 5-HT without affecting 5-HT release (Trulson, 1985).

Taken together, not only may the parameters used to calculate reductions in brain TRP availability and 5-HT metabolism be somewhat inaccurate, no direct evidence exists that the ATD-induced reductions in central 5-HT levels correlate with parallel changes in 5-HT neuronal activity. In general, therefore, ATD-induced behavioural alterations cannot easily be directly attributed to changes in 5-HT neuronal activity. This leaves the contribution of alternative mechanisms open, which is supported by the findings described in the present thesis. 


\section{Potential alternative mechanisms}

\section{Decreased nitric oxide synthase activity}

The enzyme nitric oxide synthase (NOS) catalyzes the conversion of the amino acid arginine (ARG) into citrulline (CIT) and nitric oxide (NO; Dawson and Dawson, 1996). Thus, NOS inhibition results in less conversion of ARG into CIT and NO, and the amount of NO synthesis might be directly related to the levels of ARG and CIT. Significant lower CIT levels were found in the hippocampus after TRP- treatment compared to TRP+ treatment (chapter 3), which had been previously reported by others (Lieben et al., 2004a). However, in both cases the decrease in CIT concentration appeared to be independent of changes in its precursor ARG. This suggests that a decrease in peripheral TRP might directly affect the activity of NOS, and that decreases in CIT most likely parallel decreases in NO. Endogenous $\mathrm{NO}$ can modulate neuronal function through interference with the release of several neurotransmitters (Prast and Philippu, 2001), yet its precise interaction with the 5-HT system seems rather complex. Whereas slight increases in NO concentrations most likely enhance 5-HT release, moderate increases appear to decrease 5-HT release (Kaehler et al., 1999). The modulation of 5-HT release by NO might therefore depend on pre-existing NO concentrations and the effects might be differently regulated in distinct brain areas (Smith and Whitton, 2000).

A decrease in the activity of NOS and subsequent decreased synthesis of NO after ATD could underlie the ATD-induced object memory impairments (Blokland et al., 1998). Hippocampal inhibition of NOS is known to impair object recognition performance in rats (Blokland et al., 1998; Prickaerts et al., 2002). Since the ATD-induced decrease in brain CIT levels is seemingly caused by an interruption in NOS activity, this same decrease in NOS activity presumably also decreases the synthesis of NO, which could explain the ATDinduced object recognition impairments found in the present thesis (chapter O).

The second messenger molecules cyclic adenosine monophosphate (cAMP) and cGMP play an important role in intracellular signalling and are highly involved in learning and memory processes (Bernabeu et al., 1996; Bernabeu et al., 1997; Prickaerts et al., 2002; Rutten et al., 2007b). Both cAMP and cGMP are selectively hydrolyzed by PDE enzymes, and inhibition of PDE appears to be a reliable method for improving memory processes by increasing the levels of either cAMP, cGMP or both (Blokland et al., 2006). Administration of the PDE2 inhibitor, BAY 60-7550, and the PDE5 inhibitor, zaprinast, have shown to increase NOS activity in rat hippocampus and striatum, and improve object recognition performance (Domek-Lopacinska and Strosznajder, 2008). Thus, besides directly increasing presynaptic cGMP levels, the enhancing effects of PDE5 and PDE2 inhibition upon memory performance might also be mediated by activation of NOS post-synaptically (Domek-Lopacinska and Strosznajder, 2008). Since NO can freely diffuse back into the pre-synapse, it 
can increase cGMP levels by stimulating the synthesis of soluble guanylyl cyclase (sGC) (Murad et al., 1978). An increase in NOS activity most likely increases NO concentrations, which might underlie the improvement of the ATD-induced memory impairment by the PDE2 inhibitor BAY 60-7550 and the PDE5 inhibitor vardenafil as revealed in the present thesis (chapter O).

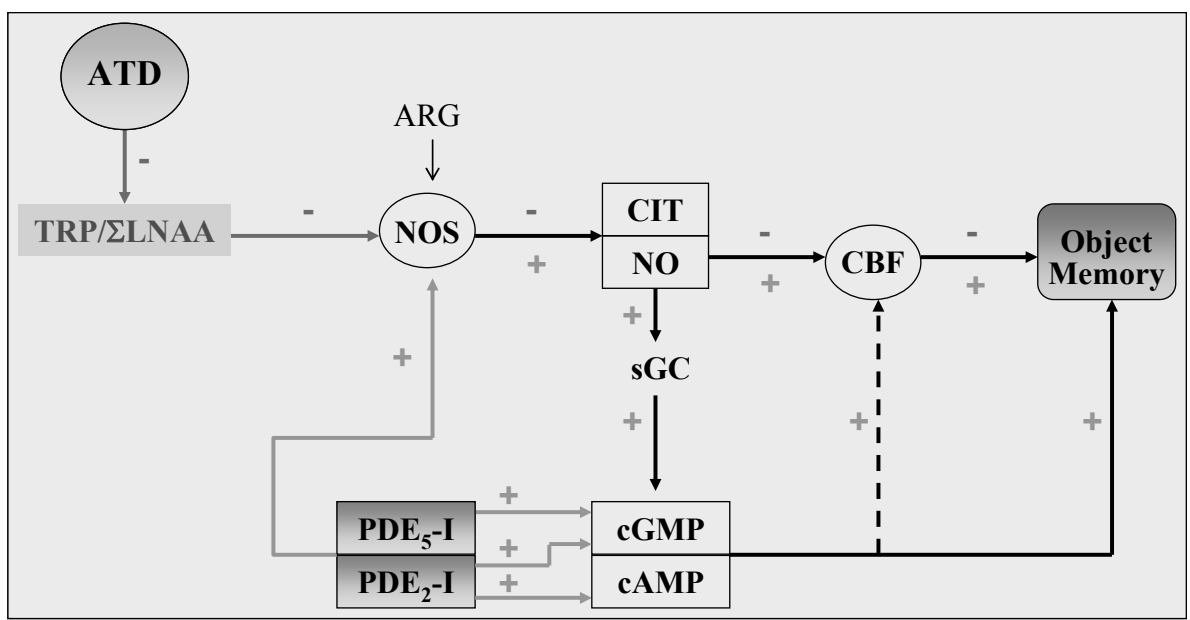

Figure 1 Potential underlying mechanism of acute tryptophan depletion-induced rat object memory impairment and its attenuation through phosphodiesterase inhibition

A decrease in the ratio of tryptophan (TRP) to the sum of other large neutral amino acids (LLNAA) induced by acute tryptophan depletion (ATD) potentially directly affects (-) the activity of nitric oxide synthase (NOS), thereby decreasing central citrulline (CIT) levels independent of changes in the concentration of its precursor arginine (ARG). A decrease in CIT most likely parallels a reduction in nitric oxide (NO) which subsequently affects local cerebral blood flow $(\mathrm{CBF})$ in brain areas highly implicated in object memory processes. Indicated with $(+)$ is the possible mechanisms behind the improvement of object memory deficits by inhibition of either the phosphodiesterase (PDE) enzyme type $2\left(\mathrm{PDE}_{2}-\mathrm{I}\right)$ or type 5 ( $\left.\mathrm{PDE}_{5}-\mathrm{I}\right)$, thereby directly increasing the levels of cyclic adenosine monophosphate (cAMP) and/or cyclic guanosine monophosphate (cGMP), respectively. Both second messenger molecules are highly implicated in learning and memory processes. Inhibition of both types of PDE also directly activates NOS, thereby increasing NO levels that stimulate the synthesis of soluble guanylyl cyclase (sGC) and cGMP pre-synaptically. The vasodilating properties of the PDE5 and PDE2 inhibitors might additionally attenuate ATD-induced decreases in CBF. (-) inhibition or decrease; $(+)$ stimulation or increase

\section{Cerebrovascular abnormalities}

Under normal physiological conditions, the cerebral metabolic rate of glucose (CMRG) provides an index of changes in regional neuronal activity, and changes in glucose metabolism are found to be closely coupled to changes in CBF (Kuschinsky, 1991; Sokoloff, 1981). On this basis, abnormalities in either of these closely linked neurophysiological parameters in depressive subjects are thought to reflect changes in serotonergic neurotransmission in brain areas that 
can be functionally linked to the complexity of depressive symptomatology displayed (Drevets et al., 1998a, 1998b; Drevets et al., 1999; Drevets, 2000). However, the 5-HT neurotransmitter is a powerful vasoconstrictor (Edvinsson and MacKenzie, 1976) and serotonergic fibers innervating cerebral arteries, arterioles and veins have been identified (Steinbusch, 1981). Thus, if depression is represented by decreased central 5-HT neurotransmitter concentrations, an increase in $\mathrm{CBF}$ would seem more likely. Likewise, ATD presumably reduces 5-HT synthesis and thus decreases vasoconstrictor tone, thereby most likely increasing $\mathrm{CBF}$ due to vasodilation. Surprisingly, a decrease in local CBF following ATD has been reported in human subjects (Talbot and Cooper, 2006) and was also observed in rats (chapter 3), where the acute decrease of peripheral TRP levels resulted in a downwards resetting of the cerebral flowmetabolism coupling relationship independently of changes in central TRP or 5-HT. This parallels preliminary findings of an uncoupling of flow from metabolism in unipolar depressed patients compared to bipolar patients and healthy controls (Dunn et al., 2005). Although controversy exists about the exact location and direction of the neurophysiological abnormalities in depressive subjects (Drevets, 2000; Soares and Mann, 1997), depressive illness is generally characterized by decreases in cerebral blood flow and glucose metabolism in limbic and prefrontal cortex structures. However, it seems unlikely that a decrease in 5-HT alone accounts for the specific decreases in haemodynamic regulation. In addition, low CBF most likely increases the interaction between the albumin-bound TRP complex and the glycocalyx of the $\mathrm{BBB}$, thereby causing more TRP to dissociate from albumin (Pardridge and Fierer, 1990; Smith et al., 1987). This might provide an explanation for the fact that ATD-induced peripheral depletion of total TRP levels does not result in significant decreases in brain TRP availability. Although it remains unclear how changes in peripheral TRP concentrations can interfere with the dynamic regulation of cerebral blood flow, the findings support the notion that the underlying mechanisms of ATD might go beyond a straightforward 5-HTmediated mechanism and that depression in general cannot be simplistically described as a 5-HT dysfunctional disorder (see also Figure 1).

In general, decreased local CBF is best explained by a loss of dilator tone. Decreased local $\mathrm{CBF}$ has also been reported after direct inhibition of endothelial or neuronal NOS (Kelly et al., 1994; Kelly et al., 1995) and, as mentioned previously, direct inhibition of NOS in the hippocampus is known to impair object memory performance. These findings, together with the ATDinduced reduction in local CBF independent of central 5-HT changes, support the suggestion that the ATD-induced impairments in object memory performance of rats is most likely ascribed to a decrease in NO after ATD. The involvement of NO can be explained by the ATD-induced changes in brain levels of CIT as described earlier. All the findings together suggest that ATDinduced object memory impairments are most likely caused by a decrease in $\mathrm{NO}$ which reduces local $\mathrm{CBF}$ in hippocampal areas highly implicated in 
memory processing. Additionally, this is in line with the fact that PDE2 and PDE5 inhibition have been found to increase NOS activity in the hippocampus, which explains their potential to attenuate object memory impairments. In addition, PDE inhibitors increase central cAMP and cGMP concentrations, which are both well known vasodilators (Dundore et al., 1993). Although no direct effects upon cerebrovascular dynamics have been found after PDE2 or PDE5 inhibition (Rutten et al., 2009), their strong vasodilating properties might contribute to the improvement of object memory performance through attenuation of the ATD-induced cerebrovascular effects.

\section{Decreased brain-derived neurotrophic factor}

The pivotal role of disrupted BDNF regulation in both depressive symptomatology and cognitive dysfunction, as well as the co-regulating mechanisms between BDNF and the 5-HT system in general, lead us to explore the effects of ATD upon peripheral and central BDNF levels. Highest levels of BDNF mRNA have been reported in the dentate gyrus and hippocampal CA3 and CA2 layers (Ernfors et al., 1990; Smith et al., 1995a) which corresponds with areas in which ATD induced decreases in local CBF were observed (dentate PO, CA3 and CA2 region of the hippocampus). Regional changes in BDNF protein levels have been found after brief cerebrovascular events (Kokaia et al., 1996), consistent with the fact that BDNF plays an important neuroprotective role (Larsson et al., 1999; Lindvall et al., 1994).

Neurotrophins also play an important role in neuronal plasticity, including long-term potentiation (LTP). As LTP is assumed to be the underlying substrate of learning and memory processes (Bliss and Collingridge, 1993), BDNF is thought to be a potential mediator of memory formation in general, and is known to be required specifically for memory consolidation (Lee et al., 2004). ATD in human subjects selectively impairs memory consolidation (Riedel et al., 1999), which has been suggested to be caused by lower 5-HT in hippocampal areas (Riedel, 2004). However, the ATD-induced cerebrovascular changes, independent of changes in central 5-HT levels, suggest that decreased $\mathrm{CBF}$ in brain regions normally high in BDNF levels might underlie BDNFmediated alterations in learning and memory processing after ATD. Moreover, ATD did not induce direct changes in peripheral or central BDNF levels (chapter 5), which has also been reported by others (Cahir et al., 2008).

\section{Kynurenine metabolites}

TRP not only leaves the plasma by uptake into the brain, but also by catabolism in the liver by TRP pyrrolase into kynurenine (KYN) and to a minor extent by excretion into the urine. KYN is the major degradation product of TRP and is further converted into potentially neuroactive metabolites such as kynurenine acid (KYNA) and quinolinic acid (QUINA; Young and Leyton, 2002). 
Independently of each other, both metabolites exert specific effects upon N-methyl-D-aspartate (NMDA) receptors (Stone, 1993), which play an important role in LTP and memory formation (Morris et al., 1990; Morris, 2003). NMDA receptor antagonists have been shown to inhibit LTP and selectively impair learning and memory (Morris et al., 1986). Whereas KYNA has a neuroprotective, antagonistic effect upon glutamatergic NMDA receptors, QUINA depolarises neurons by activating NMDA receptors (Stone and Perkins, 1981). As a result, QUINA can induce overstimulation of the NMDA receptor, thereby producing neurotoxicity and subsequent neurotoxic damage, similar to that found in hypoxia and ischemia (Schwarcz et al., 1983; Stone, 2001; Stone et al., 2007). Thus, an ATD-induced shift in the balance of KYN metabolites could provide an explanation for the observed cerebral oligaemia paralleling decreased peripheral TRP levels (chapter 3) and might additionally account for the consistently reported memory impairments after ATD, as has been suggested by others (Blokland et al., 2004). However, unpublished preliminary data do not support this hypothesis, as no changes were observed in central KYN levels or its metabolites $4 \mathrm{~h}$ after ATD in rats (Lieben, 2004).

\section{Confounding stress effects}

Together with other monoamine systems, brain 5-HT is critically involved in the mediation of the central response to stressors and subsequent behavioural adaptation (de Kloet et al., 2005). Acute stressors stimulate the hypothalamicpituitary-adrenal (HPA) axis activity, thereby increasing central 5-HT necessary for stress coping (van Praag, 2004). Overnight food deprivation, repeated oral administrations by gavage, blood sampling and the immobilization necessary for applying the former are well-known stressors (Chamas et al., 2004; Curzon et al., 1972; Jahng et al., 2007; Nakahara and Nakamura, 1999), yet are experimental procedures inevitably implicated in the application of the ATD method. Previous studies with human subjects have revealed that stress might be a critical factor in mediating alterations in the ratio TRP/ 2 LNAA in plasma and subsequent changes in behavioural performance (Markus et al., 2000a; Markus, 2007, 2008; Markus et al., 2000b). In rats, both acute and repeated exposure to stressful stimuli have been shown to increase glucocorticoid levels and alter 5-HT turnover and release in both the hippocampus and frontal cortex (Chaouloff, 1993). Thus, ATD application-related procedures might interfere with normal brain TRP metabolism and subsequent 5-HT synthesis, thereby acting as confounding stress factors for the pharmacokinetic and behavioural effects of ATD.

\section{Stress and tryptophan-albumin binding}

Evidence exists that drugs that displace TRP from its albumin binding sites increase brain TRP levels. Likewise stress-induced changes in the breakdown of 
fat stored in fat cells (lipolysis) may alter brain TRP concentrations. In the same way that insulin stimulates the uptake of albumin-bound fatty acids by fat cells, thereby decreasing the fraction of free TRP in plasma, stress can result in the reverse; stress increases lipolysis and thus the amount of plasma free-fatty acids (McMenamy, 1965). This increases the affinity of albumin for fatty-acids, thereby displacing TRP from its albumin binding sites. The resultant increase in the fraction of free TRP in plasma might increase the availability of TRP for uptake into the brain. The subsequent acute increase in brain TRP availability might be reflected upon the compensatory increase in tryptophan hydroxylase (TPH) levels, as has been demonstrated after a single exposure to immobilization stress in male Sprague Dawley rats (Chamas et al., 2004). Similarly, at a central level the combination of immobilization stress and food deprivation has been reported to increase brain TRP levels in rats to a considerable greater level than either of the stressors alone (Curzon et al., 1972). As more 5-HT is needed to be able to cope with the acute stressful situation, brain TRP levels rise to maintain adequate 5-HT concentration.

These findings strongly support the results reported in the present thesis. In C57BL/6 mice, multiple experimental procedures together significantly altered the ratio TRP/ $\Sigma$ LNAA in plasma within 20 min (Chapter 2, study 5). Whereas a significant decrease was found after food deprivation together with blood sampling, oral treatment by gavage after blood sampling and food deprivation increased the ratio TRP $/ \Sigma \mathrm{LNAA} 20 \mathrm{~min}$ after treatment. The latter combination represents the standard experimental situation and thus suggests that a profound depletion of peripheral TRP is more difficult to establish if the ratio initially increases due to acute stress of experimental procedures. In addition, decreased levels were found in prefrontal BDNF levels after overnight food deprivation together with an oral application by gavage compared to a group that was not food deprived or exposed to gavage procedures (Chapter 5). Moreover, this paralleled the lack of changes in 5-HIAA levels and the ratio 5-HIAA/5-HT in the prefrontal cortex after ATD, whereas a significant ATDinduced decrease was found in these parameters in the hippocampus where no changes in BDNF were observed. BDNF is known to be extremely stressresponsive (Smith et al., 1995b), which is supported by studies showing that both acute and repeated immobilization stress considerably decreases BDNF mRNA even within $1 \mathrm{~h}$ (Smith et al., 1995a; Ueyama et al., 1997). Thus, experimental stressors might have interfered with 5-HT metabolism through adapting BDNF levels, thereby preventing or changing the expected ATDinduced neurochemical alterations. Stress-induced changes in BDNF are not only glucocorticoid-mediated (Prickaerts et al., 2006), but might be additionally regulated by alterations in the activity of specific 5-HT receptors (Vaidya et al., 1997; Vollmayr et al., 2000). In general, acute stress effects might explain the moderate depletion found in the studies of the present thesis, and the failure to considerably reduce central 5-HT levels. 


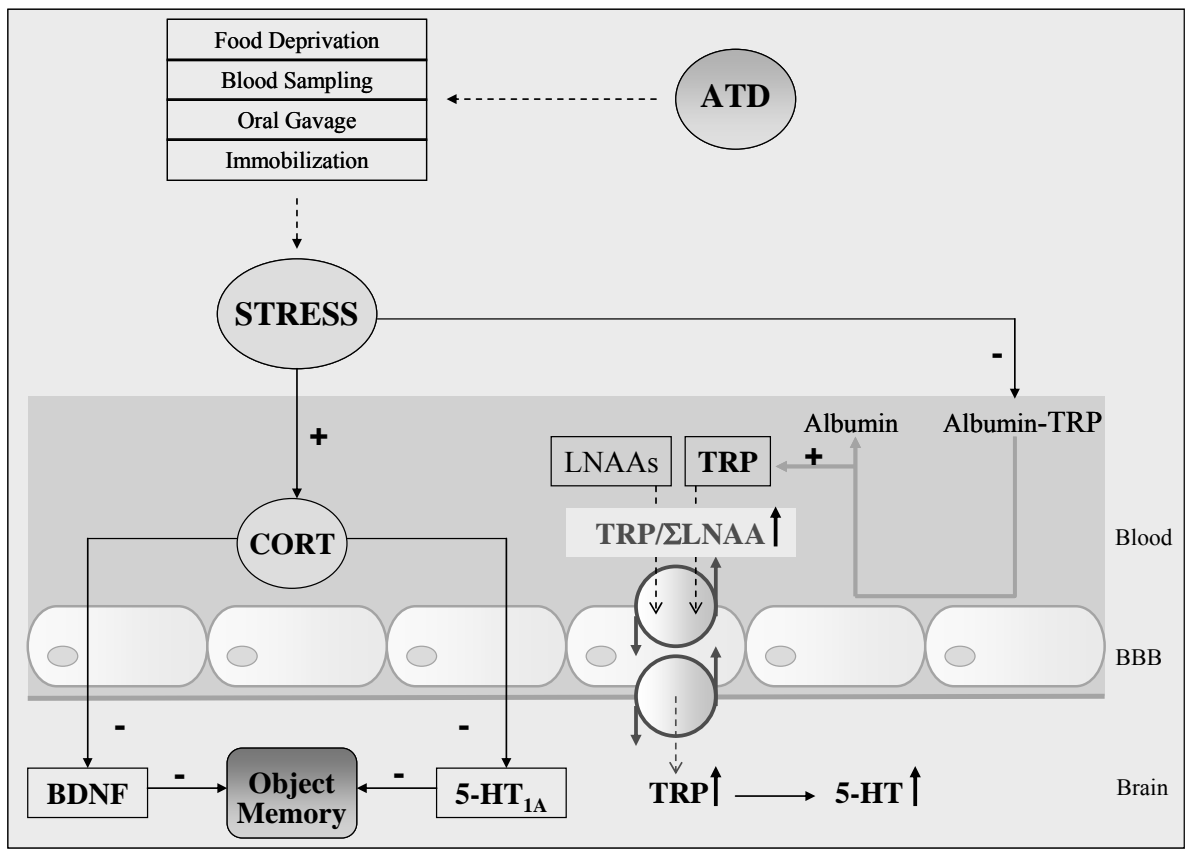

Figure 2 Potential confounding stress effects of acute tryptophan depletion application-related procedures

The method of acute tryptophan depletion (ATD) requires several highly stressful procedures, such as overnight food deprivation, repeated blood sampling, oral administrations by gavage and immobilization. Acute stress stimulates lipolysis, thereby increasing the amount of free-fatty acids in plasma that displace tryptophan (TRP) from its albumin binding sites. The resultant rise in free plasma TRP changes the ratio of TRP to the sum of the other large neutral amino acids (TRP/ $\Sigma$ LNAA) in favour of TRP, thereby increasing the amount of TRP available for uptake into the brain and its subsequent synthesis into 5-HT. Stress stimulates the secretion of corticosterone (CORT) that decreases brain-derived neurotrophic factor (BDNF) and 5-HT1A receptor binding. Both BDNF and 5-HT1A receptor functioning are highly implicated in learning and memory processes. The acute stress of ATD application-related procedures might thus explain the failure of ATD to considerably decrease 5-HT metabolism and its negative effect upon object memory performance through increased CORT levels.

The effects of acute stress, including the ATD-related application procedures, upon 5-HT autoreceptor binding might additionally provide an explanation for the consistently reported ATD-induced memory dysfunction as indicated by object recognition impairment in rats (Jans et al., 2008; Jans et al., 2007; Jans and Blokland, 2008; Lieben et al., 2004b; Rutten et al., 2007a; van Donkelaar et al., 2008). Moreover, the impairment in object memory performance seems even more pronounced in 5-HT transporter (SERT) knockout rats that are predisposed of abnormal 5-HT function (Olivier et al., 2008). Repeated exposure to food deprivation stress appears to decrease SERT mRNA levels (Jahng et al., 2007) and has also been shown to impair object memory performance (Beck and Luine, 1999). Several 5-HT receptors, including 
5- $\mathrm{HT}_{1 \mathrm{~A}}$, are known to be highly involved in memory function (King et al., 2008) and ATD has been shown to decrease $5-\mathrm{HT}_{1 \mathrm{~A}}$ receptor binding (Cahir et al., 2007). Moreover, acute corticosterone administration directly decreases 5-HT $\mathrm{HT}_{1 \mathrm{~A}}$ autoreceptor functioning (Laaris et al., 1995). Decreased SERT function is generally associated with increased stress responsivity (Canli and Lesch, 2007), which might explain why SERT knockout rats show impaired object recognition even after administration of the TRP+ control mixture (Olivier et al., 2008). Yet, untreated SERT knockout rats did not show an impairment in object memory performance, which seems to be in line with the confounding ATD-application effects revealed in the presents thesis (chapter 2 and 5). Thus, a more generalized stress effect of ATD application-related procedures, possibly linked to a decrease in $5-\mathrm{HT}_{1 \mathrm{~A}}$ receptor function as described above, might underlie the ATD-induced impaired object recognition performance for which intact $5-\mathrm{HT}_{1 \mathrm{~A}}$ receptor function seems to be crucial (see also Figure 2).

\section{Implications for cognitive and affective processes}

The 5-HT system is highly implicated in a wide range of functional processes. After mood lowering, cognitive functioning is the most prominently impaired functional process implicated in clinical depression. Whereas mood-lowering effects after ATD are only experienced in depression-free subjects predisposed to genetic 5-HT abnormalities, altered cognitive processing after ATD is observed in both healthy and genetically vulnerable subjects. Moreover, ATDinduced impaired object memory performance as measured by the object recognition task is consistently reported in rats (Jans et al., 2008; Jans et al., 2007; Jans and Blokland, 2008; Lieben et al., 2004b; Olivier et al., 2008) even after only moderate peripheral TRP depletion (Rutten et al., 2007a) or a single administration (van Donkelaar et al., 2008). For adequate cognitive processing, the 5-HT system interacts highly with other neurophysiological systems that might all be interrupted after ATD, as confirmed by the findings reported in the present thesis. This further supports the notion that an acute decrease in peripheral TRP levels directly interferes with mechanisms implicated in cognitive processing that presumably depend less upon 5-HT functioning per se. The potential alternative mechanisms underlying ATD effects might therefore provide an explanation for the consistently reported ATD-induced cognitive deficits across species.

Chronic exposure to stressful situations is one of the main contributing factors to the onset of depressive illness (van Praag, 2004) and an intact 5-HT system is critical for mediating an adequate neurophysiological stress response and subsequent behavioural adaptation (de Kloet et al., 2005). At the level of somatodendritic 5-HT autoreceptors, adaptations to stress might be reflected by a reduction of these receptors, thereby reducing serotonergic feedback in 
dorsal raphe projection areas in an attempt to counteract an ATD-induced decrease in central 5-HT (Cahir et al., 2007). Such an adaptive mechanism to an acute stressor might be less efficient in subjects predisposed to 5-HT dysfunction, which might account for the mood-lowering effects after ATD in these subjects.

Both 5- $\mathrm{HT}_{1 \mathrm{~A}}$ and $5-\mathrm{HT}_{2 \mathrm{~A}}$ receptors are highly implicated in the mechanism of action of many antidepressants (Cryan and Leonard, 2000). One of the most remarkable effects of ATD in human subjects is that it only induces a depressive relapse in medication-free, remitted, depressed patients with a positive treatment history of serotonergic antidepressants. Antidepressant treatment with either selective serotonin re-uptake inhibitors (SSRIs) or MAO inhibitors (MAO-Is) are generally associated with a decrease in 5- $\mathrm{HT}_{2}$ receptors (Cryan and Leonard, 2000; Landen and Thase, 2006), whereas depression has been associated with an up-regulation of these 5-HT receptors. Thus, in general, the change in postsynaptic $5-\mathrm{HT}_{2}$ receptors (density or binding sites), caused either by a decrease in 5-HT release or representing stress-induced adaptations, could be responsible for a subject's experience of depressive symptoms. Although, ATD does not significantly decrease $5-\mathrm{HT}_{2 \mathrm{~A}}$ receptor binding in adult male rats, a positron emission tomography (PET) study in humans has revealed decreased 5- $\mathrm{HT}_{2}$ binding in female subjects (Yatham et al., 2001). This might be in line with sex-specific changes in the number of binding sites that have been reported in rats and might link sex differences in peripheral hormonal secretion with higher emotional disturbances in female gender (Fischette et al., 1983). Likewise, lower 5-HT synthesis rates have been observed after ATD in female as compared with male human subjects (Nishizawa et al., 1997). In general, these specific effects of ATD upon 5-HT receptor types might provide an explanation for the lack of ATD-induced mood-lowering effects in healthy subjects without genetic predisposition to 5-HT dysfunction, and for the fact that these effects in remitted depressed patients depend upon the type of antidepressant treatment.

Since corticosteroid modulation of 5-HT function plays a central role in mood disorders, and cognitive functioning is highly impaired in clinical depression, the ATD findings reported in the present thesis might have important implications regarding the mechanisms of adaptation to stress, and the implication of the 5-HT system in cognitive processing. As the 5-HT system is primarily modulatory, it is not surprising that in the clinical situation, most 5-HT-based treatments result in only a partial symptomatic improvement. The present findings support new insights for alternative treatment strategies along other pathways that interact highly with the 5-HT system. Up-regulation of the cAMP and BDNF systems has already resulted in a novel model for the mechanism of action of antidepressants and new targets for the development of therapeutic targets (Duman, 1998) 


\section{In Conclusion}

Depression is characterized by sustained sadness along with psychological, behavioural and physical symptoms. On average, about 800.000 people commit suicide every year, of which more than half are aged between 15 and 44 years old. It is ranked as the seventh most important cause of disease burden in lowand middle-income countries, as it tends to be disabling, recurring or longlasting, and often remains untreated. The ATD method seems important in the investigation of 5-HT-related vulnerability factors implicated in the onset of depression and previously the monoamine systems were considered to be primarily responsible for the onset of depressive disorders. However, the lack of mood-lowering effects after ATD in healthy subjects does not support a direct causal relationship between acute decreased 5-HT metabolism and major depressive disorder. Moreover, the findings of the present thesis support the notion that ATD possibly exerts its neurochemical and behavioural effects through other mechanisms that might go beyond a straightforward decrease in 5-HT metabolism. These mechanisms should be taken into account for an adequate interpretation of data resulting from application of the method in both clinical and preclinical settings. Abnormal physiological conditions, including ATD-application related confounding stressors possibly interfere with the high number of cellular processes that are involved in 5-HT synthesis and metabolism, and might trigger other neurophysiological systems that generally interact with the 5-HT system. This possibly also reflects the heterogeneity of major depressive disorder in general, as all these neurophysiological processes might eventually be altered in clinical conditions and thus potentially be treated along different pharmacological pathways. In general, the findings of this thesis support the fact that depression may not be caused solely by an abnormality of 5-HT function, but more likely by a dysfunction of other systems or brain regions modulated by 5-HT or interacting with its dietary precursor. Likewise, the acute tryptophan depletion method does not seem to challenge the 5-HT system per se, but rather triggers 5-HT-mediated adverse events. A degree of caution should therefore be maintained in extrapolating the results of ATD studies to clinical depression.

\section{References}

Ardis TC, Cahir M, Elliott JJ, Bell R, Reynolds GP, Cooper SJ (2009). Effect of acute tryptophan depletion on noradrenaline and dopamine in the rat brain. J Psychopharmacol 23(1): 51-55.

Badawy AB (2009). Plasma free tryptophan revisited: what you need to know and do before measuring it. J Psychopharmacol.

Beck KD, Luine VN (1999). Food deprivation modulates chronic stress effects on object recognition in male rats: role of monoamines and amino acids. Brain Res 830(1): 56-71.

Bel N, Artigas F (1996). Reduction of serotonergic function in rat brain by tryptophan depletion: effects in control and fluvoxamine-treated rats. J Neurochem 67(2): 669-676. 
Bernabeu R, Schmitz P, Faillace MP, Izquierdo I, Medina JH (1996). Hippocampal cGMP and cAMP are differentially involved in memory processing of inhibitory avoidance learning. NeuroReport 7(2): 585-588.

Bernabeu R, Schroder N, Quevedo J, Cammarota M, Izquierdo I, Medina JH (1997). Further evidence for the involvement of a hippocampal cGMP/cGMP-dependent protein kinase cascade in memory consolidation. NeuroReport 8(9-10): 2221-2224.

Bliss TV, Collingridge GL (1993). A synaptic model of memory: long-term potentiation in the hippocampus. Nature 361(6407): 31-39.

Blokland A, Prickaerts J, Honig W, de Vente J (1998). State-dependent impairment in object recognition after hippocampal NOS inhibition. Neuroreport 9(18): 4205-4208.

Blokland A, Lieben C, Deutz NEP, Schmitt J (2004). Acute Tryptophan depletion: comparing the effects of an amino acid mixture with a gelatin-based protein in man and rats. Current Topics in Nutraceutical Research 2(3): 1-8.

Blokland A, Schreiber R, Prickaerts J (2006). Improving memory: a role for phosphodiesterases. Curr Pharm Des 12(20): 2511-2523.

Boadle-Biber MC (1993). Regulation of serotonin synthesis. Prog Biophys Mol Biol 60(1): 1-15.

Brown CM, Fletcher PJ, Coscina DV (1998). Acute amino acid loads that deplete brain serotonin fail to alter behavior. Pharmacology, biochemistry, and behavior 59(1): 115-121.

Cahir M, Ardis T, Reynolds GP, Cooper SJ (2007). Acute and chronic tryptophan depletion differentially regulate central 5-HT1A and 5-HT $2 \mathrm{~A}$ receptor binding in the rat. Psychopharmacology 190(4): 497-506.

Cahir M, Ardis TC, Elliott JJ, Kelly CB, Reynolds GP, Cooper SJ (2008). Acute tryptophan depletion does not alter central or plasma brain-derived neurotrophic factor in the rat. Eur Neuropsychopharmacol 18(5): 317-322.

Canli T, Lesch KP (2007). Long story short: the serotonin transporter in emotion regulation and social cognition. Nat Neurosci 10(9): 1103-1109.

Chamas FM, Underwood MD, Arango V, Serova L, Kassir SA, Mann JJ, Sabban EL (2004). Immobilization stress elevates tryptophan hydroxylase mRNA and protein in the rat raphe nuclei. Biol Psychiatry 55(3): 278-283.

Chaouloff F (1993). Physiopharmacological interactions between stress hormones and central serotonergic systems. Brain Res Brain Res Rev 18(1): 1-32.

Cryan JF, Leonard BE (2000). 5-HT1A and beyond: the role of serotonin and its receptors in depression and the antidepressant response. Hum Psychopharmacol 15(2): 113-135.

Curzon G, Joseph MH, Knott PJ (1972). Effects of immobilization and food deprivation on rat brain tryptophan metabolism. J Neurochem 19(8): 1967-1974.

Dawson VL, Dawson TM (1996). Nitric oxide actions in neurochemistry. Neurochem Int 29(2): 97-110.

de Kloet ER, Joels M, Holsboer F (2005). Stress and the brain: from adaptation to disease. Nat Rev Neurosci 6(6): 463-475.

Domek-Lopacinska K, Strosznajder JB (2008). The effect of selective inhibition of cyclic GMP hydrolyzing phosphodiesterases 2 and 5 on learning and memory processes and nitric oxide synthase activity in brain during aging. Brain Res 1216: 68-77.

Drevets WC, Ongur D, Price JL (1998a). Reduced glucose metabolism in the subgenual prefrontal cortex in unipolar depression. Mol Psychiatry 3(3): 190-191.

Drevets WC, Ongur D, Price JL (1998b). Neuroimaging abnormalities in the subgenual prefrontal cortex: implications for the pathophysiology of familial mood disorders. Mol Psychiatry 3(3): 220-226, 190-191.

Drevets WC, Frank E, Price JC, Kupfer DJ, Holt D, Greer PJ, Huang Y, Gautier C, Mathis C (1999). PET imaging of serotonin $1 \mathrm{~A}$ receptor binding in depression. Biol Psychiatry 46(10): 1375-1387.

Drevets WC (2000). Neuroimaging studies of mood disorders. Biol Psychiatry 48(8): 813-829.

Duman RS (1998). Novel therapeutic approaches beyond the serotonin receptor. Biol Psychiatry 44(5): 324-335. 
Dundore RL, Clas DM, Wheeler LT, Habeeb PG, Bode DC, Buchholz RA, Silver PJ, Pagani ED (1993). Zaprinast increases cyclic GMP levels in plasma and in aortic tissue of rats. Eur J Pharmacol 249(3): 293-297.

Dunn RT, Willis MW, Benson BE, Repella JD, Kimbrell TA, Ketter TA, Speer AM, Osuch EA, Post RM (2005). Preliminary findings of uncoupling of flow and metabolism in unipolar compared with bipolar affective illness and normal controls. Psychiatry Res 140(2): 181-198.

Edvinsson L, MacKenzie ET (1976). Amine mechanisms in the cerebral circulation. Pharmacol Rev 28(4): 275-348.

Ernfors P, Wetmore C, Olson L, Persson H (1990). Identification of cells in rat brain and peripheral tissues expressing mRNA for members of the nerve growth factor family. Neuron 5(4): 511-526.

Fadda F, Cocco S, Stancampiano R (2000). A physiological method to selectively decrease brain serotonin release. Brain Res Brain Res Protoc 5(3): 219-222.

Fernstrom JD, Hirsch MJ (1977). Brain serotonin synthesis: reduction in corn-malnourished rats. J Neurochem 28(4): 877-979.

Fischette CT, Biegon A, McEwen BS (1983). Sex differences in serotonin 1 receptor binding in rat brain. Science 222(4621): 333-335.

Jahng JW, Kim JG, Kim HJ, Kim BT, Kang DW, Lee JH (2007). Chronic food restriction in young rats results in depression- and anxiety-like behaviors with decreased expression of serotonin reuptake transporter. Brain Res 1150: 100-107.

Jans L, Korte-Bouws G, Korte S, Blokland A (2008). The effects of acute tryptophan depletion on affective behaviour and cognition in Brown Norway and Sprague Dawley rats. J Psychopharmacol.

Jans LA, Lieben CK, Blokland A (2007). Influence of sex and estrous cycle on the effects of acute tryptophan depletion induced by a gelatin-based mixture in adult Wistar rats. Neuroscience 147(2): 304-317.

Jans LA, Blokland A (2008). Influence of chronic mild stress on the behavioural effects of acute tryptophan depletion induced by a gelatin-based mixture. Behav Pharmacol 19(7): 706-715.

Kaehler ST, Singewald N, Sinner C, Philippu A (1999). Nitric oxide modulates the release of serotonin in the rat hypothalamus. Brain Res 835(2): 346-349.

Kelly PA, Thomas CL, Ritchie IM, Arbuthnott GW (1994). Cerebrovascular autoregulation in response to hypertension induced by NG-nitro-L-arginine methyl ester. Neuroscience 59(1): 13-20.

Kelly PA, Ritchie IM, Arbuthnott GW (1995). Inhibition of neuronal nitric oxide synthase by 7 nitroindazole: effects upon local cerebral blood flow and glucose use in the rat. J Cereb Blood Flow Metab 15(5): 766-773.

King MV, Marsden CA, Fone KC (2008). A role for the 5-HT(1A), 5-HT(4) and 5-HT(6) receptors in learning and memory. Trends Pharmacol Sci 29(9): 482-492.

Kokaia Z, Nawa H, Uchino H, Elmer E, Kokaia M, Carnahan J, Smith ML, Siesjo BK, Lindvall O (1996). Regional brain-derived neurotrophic factor mRNA and protein levels following transient forebrain ischemia in the rat. Brain Res Mol Brain Res 38(1): 139-144.

Kuschinsky W (1991). Coupling of function, metabolism, and blood flow in the brain. Neurosurg Rev 14(3): 163-168.

Laaris N, Haj-Dahmane S, Hamon M, Lanfumey L (1995). Glucocorticoid receptor-mediated inhibition by corticosterone of 5-HT1A autoreceptor functioning in the rat dorsal raphe nucleus. Neuropharmacology 34(9): 1201-1210.

Landen M, Thase ME (2006). A model to explain the therapeutic effects of serotonin reuptake inhibitors: the role of 5-HT2 receptors. Psychopharmacol Bull 39(1): 147-166.

Larsson E, Nanobashvili A, Kokaia Z, Lindvall O (1999). Evidence for neuroprotective effects of endogenous brain-derived neurotrophic factor after global forebrain ischemia in rats. J Cereb Blood Flow Metab 19(11): 1220-1228.

Lee JL, Everitt BJ, Thomas KL (2004). Independent cellular processes for hippocampal memory consolidation and reconsolidation. Science 304(5672): 839-843. 
Lieben CK, Blokland A, Westerink B, Deutz NE (2004a). Acute tryptophan and serotonin depletion using an optimized tryptophan-free protein-carbohydrate mixture in the adult rat. Neurochem Int 44(1): 9-16.

Lieben CK, van Oorsouw K, Deutz NE, Blokland A (2004b). Acute tryptophan depletion induced by a gelatin-based mixture impairs object memory but not affective behavior and spatial learning in the rat. Behav Brain Res 151(1-2): 53-64.

Lieben CKJ. 2004. Serotonin and behavior: animal studies applying the method of acute tryptophan depletion (PhD thesis. Maastricht, the Netherlands: Department of Psychiatry and Neuropsychology, Maastricht University.

Lindvall O, Kokaia Z, Bengzon J, Elmer E, Kokaia M (1994). Neurotrophins and brain insults. Trends Neurosci 17(11): 490-496.

Markus CR, Olivier B, Panhuysen GE, Van Der Gugten J, Alles MS, Tuiten A, Westenberg HG, Fekkes D, Koppeschaar HF, de Haan EE (2000a). The bovine protein alpha-lactalbumin increases the plasma ratio of tryptophan to the other large neutral amino acids, and in vulnerable subjects raises brain serotonin activity, reduces cortisol concentration, and improves mood under stress. Am J Clin Nutr 71(6): 1536-1544.

Markus CR (2007). Effects of carbohydrates on brain tryptophan availability and stress performance. Biol Psychol 76(1-2): 83-90.

Markus CR (2008). Dietary amino acids and brain serotonin function; implications for stressrelated affective changes. Neuromolecular Med 10(4): 247-258.

Markus R, Panhuysen G, Tuiten A, Koppeschaar H (2000b). Effects of food on cortisol and mood in vulnerable subjects under controllable and uncontrollable stress. Physiol Behav 70(3-4): 333-342.

McMenamy RH (1965). Binding of indole analogues to human serum albumin. Effects of fatty acids. J Biol Chem 240(11): 4235-4243.

Morris RG, Anderson E, Lynch GS, Baudry M (1986). Selective impairment of learning and blockade of long-term potentiation by an N-methyl-D-aspartate receptor antagonist, AP5. Nature 319(6056): 774-776.

Morris RG, Davis S, Butcher SP (1990). Hippocampal synaptic plasticity and NMDA receptors: a role in information storage? Philos Trans R Soc Lond, B, Biol Sci 329(1253): 187-204.

Morris RG (2003). Long-term potentiation and memory. Philos Trans R Soc Lond, B, Biol Sci 358(1432): 643-647.

Murad F, Mittal CK, Arnold WP, Katsuki S, Kimura H (1978). Guanylate cyclase: activation by azide, nitro compounds, nitric oxide, and hydroxyl radical and inhibition by hemoglobin and myoglobin. Adv Cyclic Nucleotide Res 9: 145-158.

Nakahara D, Nakamura M (1999). Differential effect of immobilization stress on in vivo synthesis rate of monoamines in medial prefrontal cortex and nucleus accumbens of conscious rats. Synapse 32(3): 238-242.

Nishizawa S, Benkelfat C, Young SN, Leyton M, Mzengeza S, de Montigny C, Blier P, Diksic M (1997). Differences between males and females in rates of serotonin synthesis in human brain. Proc Natl Acad Sci U S A 94(10): 5308-5313.

Oldendorf WH, Szabo J (1976). Amino acid assignment to one of three blood-brain barrier amino acid carriers. Am J Physiol 230(1): 94-98.

Olivier JD, Jans LA, Korte-Bouws GA, Korte SM, Deen PM, Cools AR, Ellenbroek BA, Blokland A (2008). Acute tryptophan depletion dose dependently impairs object memory in serotonin transporter knockout rats. Psychopharmacology (Berl) 200(2): 243-254.

Pardridge WM, Fierer G (1990). Transport of tryptophan into brain from the circulating, albumin-bound pool in rats and in rabbits. J Neurochem 54(3): 971-976.

Pardridge WM (1998). Blood-brain barrier carrier-mediated transport and brain metabolism of amino acids. Neurochemical research 23(5): 635-644.

Prast H, Philippu A (2001). Nitric oxide as modulator of neuronal function. Prog Neurobiol 64(1): 51-68. 
Prickaerts J, de Vente J, Honig W, Steinbusch HW, Blokland A (2002). cGMP, but not cAMP, in rat hippocampus is involved in early stages of object memory consolidation. Eur J Pharmacol 436(1-2): 83-87.

Prickaerts J, van den Hove DL, Fierens FL, Kia HK, Lenaerts I, Steckler T (2006). Chronic corticosterone manipulations in mice affect brain cell proliferation rates, but only partly affect BDNF protein levels. Neurosci Lett 396(1): 12-16.

Riedel WJ, Klaassen T, Deutz NE, van Someren A, van Praag HM (1999). Tryptophan depletion in normal volunteers produces selective impairment in memory consolidation. Psychopharmacology (Berl) 141(4): 362-369.

Riedel WJ (2004). Cognitive changes after acute tryptophan depletion: what can they tell us? Psychol Med 34(1): 3-8.

Rutten K, Lieben C, Smits L, Blokland A (2007a). The PDE4 inhibitor rolipram reverses object memory impairment induced by acute tryptophan depletion in the rat. Psychopharmacology (Berl) 192(2): 275-282.

Rutten K, Prickaerts J, Hendrix M, van der Staay FJ, Sik A, Blokland A (2007b). Time-dependent involvement of cAMP and cGMP in consolidation of object memory: studies using selective phosphodiesterase type 2, 4 and 5 inhibitors. Eur J Pharmacol 558(1-3): 107-112.

Rutten K, van Donkelaar EL, Ferrington L, Blokland A, Bollen E, Steinbusch HW, Kelly PA, Prickaerts JH (2009). Phosphodiesterase Inhibitors Enhance Object Memory Independent of Cerebral Blood Flow and Glucose Utilization in Rats. Neuropsychopharmacology.

Schwarcz R, Whetsell WO, Jr., Mangano RM (1983). Quinolinic acid: an endogenous metabolite that produces axon-sparing lesions in rat brain. Science 219(4582): 316-318.

Shannon NJ, Gunnet JW, Moore KE (1986). A comparison of biochemical indices of 5hydroxytryptaminergic neuronal activity following electrical stimulation of the dorsal raphe nucleus. J Neurochem 47(3): 958-965.

Smith JC, Whitton PS (2000). Nitric oxide modulates N-methyl-D-aspartate-evoked serotonin release in the raphe nuclei and frontal cortex of the freely moving rat. Neurosci Lett 291(1): 5-8.

Smith MA, Makino S, Kvetnansky R, Post RM (1995a). Stress and glucocorticoids affect the expression of brain-derived neurotrophic factor and neurotrophin-3 mRNAs in the hippocampus. J Neurosci 15(3 Pt 1): 1768-1777.

Smith MA, Makino S, Kvetnansky R, Post RM (1995b). Effects of stress on neurotrophic factor expression in the rat brain. Ann N Y Acad Sci 771: 234-239.

Smith QR, Momma S, Aoyagi M, Rapoport SI (1987). Kinetics of neutral amino acid transport across the blood-brain barrier. J Neurochem 49(5): 1651-1658.

Smith QR (2000). Transport of glutamate and other amino acids at the blood-brain barrier. J Nutr 130(4S Suppl): 1016S-1122S.

Soares JC, Mann JJ (1997). The functional neuroanatomy of mood disorders. J Psychiatr Res 31(4): 393-432.

Sokoloff L (1981). Relationships among local functional activity, energy metabolism, and blood flow in the central nervous system. Fed Proc 40(8): 2311-2316.

Stancampiano R, Melis F, Sarais L, Cocco S, Cugusi C, Fadda F (1997). Acute administration of a tryptophan-free amino acid mixture decreases 5 -HT release in rat hippocampus in vivo. Am J Physiol 272(3 Pt 2): R991-994.

Steinbusch HW (1981). Distribution of serotonin-immunoreactivity in the central nervous system of the rat-cell bodies and terminals. Neuroscience 6(4): 557-618.

Stone TW, Perkins MN (1981). Quinolinic acid: a potent endogenous excitant at amino acid receptors in CNS. Eur J Pharmacol 72(4): 411-412.

Stone TW (1993). Neuropharmacology of quinolinic and kynurenic acids. Pharmacol Rev 45(3): 309-379.

Stone TW (2001). Endogenous neurotoxins from tryptophan. Toxicon 39(1): 61-73.

Stone TW, Forrest CM, Mackay GM, Stoy N, Darlington LG (2007). Tryptophan, adenosine, neurodegeneration and neuroprotection. Metab Brain Dis 22(3-4): 337-352. 
Talbot PS, Cooper SJ (2006). Anterior cingulate and subgenual prefrontal blood flow changes following tryptophan depletion in healthy males. Neuropsychopharmacology 31(8): 17571767.

Trulson ME (1985). Dietary tryptophan does not alter the function of brain serotonin neurons. Life Sci 37(11): 1067-1072.

Ueyama T, Kawai Y, Nemoto K, Sekimoto M, Tone S, Senba E (1997). Immobilization stress reduced the expression of neurotrophins and their receptors in the rat brain. Neurosci Res 28(2): 103-110.

Vaidya VA, Marek GJ, Aghajanian GK, Duman RS (1997). 5-HT2A receptor-mediated regulation of brain-derived neurotrophic factor mRNA in the hippocampus and the neocortex. J Neurosci 17(8): 2785-2795.

van der Plasse G, Meerkerk DT, Lieben CK, Blokland A, Feenstra MG (2007). Lack of evidence for reduced prefrontal cortical serotonin and dopamine efflux after acute tryptophan depletion. Psychopharmacology (Berl) 195(3): 377-385.

van Donkelaar EL, Rutten K, Blokland A, Akkerman S, Steinbusch HW, Prickaerts J (2008). Phosphodiesterase 2 and 5 inhibition attenuates the object memory deficit induced by acute tryptophan depletion. Eur J Pharmacol 600(1-3): 98-104.

van Praag HM (2004). Can stress cause depression? Prog Neuropsychopharmacol Biol Psychiatry 28(5): 891-907.

Vollmayr B, Keck S, Henn FA, Schloss P (2000). Acute stress decreases serotonin transporter mRNA in the raphe pontis but not in other raphe nuclei of the rat. Neurosci Lett 290(2): 109-112.

Yatham LN, Liddle PF, Shiah IS, Lam RW, Adam MJ, Zis AP, Ruth TJ (2001). Effects of rapid tryptophan depletion on brain 5-HT(2) receptors: a PET study. Br J Psychiatry 178: 448-453.

Young SN, Leyton M (2002). The role of serotonin in human mood and social interaction. Insight from altered tryptophan levels. Pharmacology, biochemistry, and behavior 71(4): 857865. 


\section{Summary}

The method of acute tryptophan depletion (ATD) currently represents the most extensively applied challenge test to investigate the involvement of the serotonin (5-hydroxytryptamine; 5-HT) system and 5-HT-related vulnerability factors in the pathogenesis and pathophysiology of depression. ATD consists of manipulating the availability of the essential amino acid tryptophan (TRP), the dietary 5-HT precursor through administration of a TRP-free diet. However, the exact mechanism by which ATD exerts its neurophysiological effects, and to what extent changes in 5-HT neuronal activity contribute to ATD-induced neurochemical and behavioural alterations, is not fully clear. This impedes an adequate interpretation of the results arising from application of the method in both human and animal studies. The present thesis aimed to explore potential alternative mechanisms by which ATD exerts its neurophysiological and behavioural effects by administering rodents a gelatin-based proteincarbohydrate nutritional mixture.

Chapter 1 introduces the biochemical rationale behind the method of ATD by describing the specific features involved in the biosynthesis of the essential amino acid TRP from food into the modulating 5-HT neurotransmitter in the brain. Functions and characteristics of the 5-HT system are outlined in general and with respect to its implication in major depressive disorder. An overview is given of the utility of the ATD method in depression research with human subjects and preclinical data arisen from applying the method in rats are thoroughly reviewed. The basic mechanism behind the induction of peripheral TRP depletion by administrating a TRP-free diet is described and the validity of parameters used for interpreting the biochemical effects of ATD in the brain is critically assessed. Thereafter, the aims of the thesis to explore potential alternative mechanisms underlying the ATD method and its potential to induce affective and cognitive behavioural changes are introduced.

Chapter 2 provides an overview of multiple studies in which the pharmacokinetic and behavioural effects of different dosing regimes of the TRP-free protein-carbohydrate mixture were explored in the Swiss and particularly C57BL/6 mice. As pre-existing dysfunctional 5-HT systems are nowadays widely modelled in mice, the use of mice in stead of rats for studying behaviour in general and the implication of neurotransmitter systems in psychiatric disorders in particular has significantly increased. It was, therefore, initially aimed to establish the ATD method in the mouse for subsequent potential application as 5-HT challenge tool in genetic mice models of depression. Peripheral amino acids were measured and central TRP and 5-HT concentrations were compared with anxiety and depression-like behaviour in the elevated zero-maze (EZM), forced swimming test (FST) and tail suspension test (TST). The extent of peripheral TRP depletion highly depended upon the 
specificity of the dosing volume and the time-frame of the multiple oral administrations. While ATD resulted in a $74 \%$ reduction of the plasma ratio TRP to the sum of other large neutral amino acids (TRP/ $L \mathrm{LNAA}$ ) in Swiss mice $1 \mathrm{~h}$ after administration $(2 \times 10 \mathrm{ml} / \mathrm{kg}, 30 \mathrm{~min}$ interval), there was only a $40 \%$ reduction in C57BL/ 6 mice. The latter did not show anxiety in the EZM or increased immobility in the FST or TST. A higher dose $(2 \times 20 \mathrm{ml} / \mathrm{kg})$ with a longer interval (60 min) reduced the ratio with 68\% in C57BL/6 mice, lowered hippocampal 5-HT turnover, but had no functional effects when tested in the EZM and FST. Extreme differences in ATD effects were observed between strains and none of the profound peripheral TRP depletions paralleled central TRP or 5-HT changes. Yet, interfering ATD application-related stress effects were found to confound the ATD-induced effects upon the ratio TRP/ LLNAA in plasma. These findings have important implications for the use of ATD in general and in particular for its application in mice. It has been postulated that mice with pre-existing 5-HT dysfunction might be more vulnerable to ATD, which remains to be elucidated.

Chapter 3 describes the effects of ATD upon local cerebral blood flow (CBF) and cerebral metabolic rate of glucose (CMRG) as measured in 43 distinct brain regions of adult male Wistar rats by means of $\left[{ }^{14} \mathrm{C}\right]$-iodoantipyrine and $\left[{ }^{14} \mathrm{C}\right]-2$ deoxyglucose quantitative autoradiographic imaging, respectively. To gain more inside into the potential mechanisms underlying the ATD-induced neurochemical and behavioural effects in general, explorative experiments were continued in the established rat model of ATD. As the 5-HT neurotransmitter possesses important vasoactive properties, an acute decrease in central 5-HT concentration could alter CMRG and $\mathrm{CBF}$ and the normal relationship between them. Such cerebrovascular alterations might contribute to the underlying mechanisms of ATD-induced affective behavioural changes and cognitive impairments. Whereas the ratio TRP/ $\Sigma$ LNAA decreased with $40 \%$, no ATD-induced measurable effects upon TRP and 5-HT levels in the brain could be revealed. Nevertheless, ATD significantly reduced local CBF in 11 of the 43 brain ROIs measured, while local CMRG remained unchanged. Moreover, a downwards resetting of the relationship CBF to CMRG was found after ATD compared to the TRP+ control group, indicating a state of relative cerebrovascular oligaemia. The ATD-induced decrease in peripheral TRP levels, thus, resulted in a decrease of local CBF and an uncoupling of flow from metabolism without affecting central TRP or 5-HT levels. Therefore, a more additive role for a dysfunctional 5-HT system as an underlying mechanism of the ATD method might be implied.

Chapter 4 reveals the cerebrovascular effects of ATD in an animal model of acquired 5-HT dysfunction induced by repeated 3,4-methylenedioxymethamphetamine (MDMA; 'ecstasy') administrations. The neurotoxicity of MDMA is known to cause significant 5-HT terminal depletion and an acute decrease in 
brain 5-HT after ATD is likely to result in disturbed affective behaviour only if there is pre-existing vulnerability of the 5-HT system. By using quantitative autoradiographic imaging the effects of MDMA pretreatment were measured upon local $\mathrm{CBF}$ and CMRG under an ATD challenged condition, thereby investigating the relationship between cerebrovascular function and 5-HT changes in general and MDMA-induced 5-HT dysfunction as a vulnerability factor for depression in general and cognitive dysfunction in particular. MDMA induced significant 5-HT neurotoxicity and decreased central TRP and 5-HT levels considerably. ATD resulted in a mean $40 \%$ reduction of the ratio TRP/ $/$ LNAA in plasma without affecting central TRP or 5-HT concentrations. A downwards resetting of the relationship of CBF to CMRG was only observed after ATD treatment in the drug-naïve control animals. This indicates that ATD induced a significant upwards resetting of this relationship after MDMA pretreatment compared to controls, thereby inducing a potentiation of the MDMA-induced cerebral vascular dysfunction. ATD, thus, produces a degree of cerebrovascular hyperperfusion in animals pretreated with MDMA which could indicate a predisposition to cerebrovascular accidents, in particular haemorrhagic stroke.

Chapter 5 shows the effects of ATD upon peripheral and central concentrations of brain-derived neurotrophic factor (BDNF) in the rat while controlling for possible confounding stress effects of the specific experimental procedures. BDNF is strongly linked to the 5-HT system and, besides being extremely stress-responsive, plays an essential role in mood and memory processes and might thus contribute to the underlying mechanisms of ATDinduced behavioural changes. A significant 56\% depletion of the ratio TRP/ $/$ LNAA was found after ATD, however, 5-HT metabolism seemed only moderately affected in the hippocampus without measurable changes in central TRP or 5-HT concentrations. Although no direct effects of ATD upon serum or brain BDNF concentrations were found, a stress-mediated decrease in BDNF levels was observed in the prefrontal cortex. Moreover, brain TRP levels correlated positively with BDNF in both the prefrontal cortex and hippocampus. Thus, BDNF-mediated mechanisms due to ATD and/or its application stress might underlie ATD-induced neurochemical and behavioural alterations.

Chapter 6 examines the ability of phosphodiesterase (PDE) inhibitors to reverse an ATD-induced impairment in rat object recognition performance. Whereas affective behavioural changes after ATD remain controversial, ATDinduced memory deficits are consistently reported in humans, but also in rats as measured by means of the rat object recognition task. Inhibition of phosphodiesterase (PDE) enzymes appears to be a reliable method to improve memory processes by increasing the levels of the second messenger molecules cyclic adenosine monophosphate (cAMP) and cyclic guanosine monophosphate 
(cGMP) or both. Exploring the pro-cognitive effects of PDE inhibitors might provide more insight into potential interaction between second messenger molecules and the 5-HT system as underlying mechanism for ATD-induced cognitive dysfunction. ATD significantly decreased the ratio TRP/ $L$ LNAA in plasma and impaired object memory performance considerably. Both the PDE5 inhibitor, vardenafil, and the PDE2 inhibitor, BAY 60-7550, that increase cGMP and both cAMP and cGMP, respectively, were able to acutely improve short-term object recognition performance after an ATD-induced deficit.

Chapter 7 summarizes the results of the studies described in this thesis and provides a critical discussion concerning potential alternative mechanisms underlying the neurochemical and behavioural effects of the method of ATD. It is generally concluded that the parameters used to calculate reductions in brain TRP availability and 5-HT metabolism may be somewhat inaccurate. Therefore, like depression itself, ATD-induced behavioural alterations are not easily attributed directly to changes in 5-HT neurotransmission. The findings of the present thesis support the contribution of alternative mechanisms such as decreased nitric oxide synthase activity and cerebrovascular abnormalities that might be implicated in the behavioural effects of ATD and could also explain 5-HT-mediated clinical depressive symptoms. Moreover, experimental procedures related to the application of the ATD method seem highly stressful and potentially interfere with TRP metabolism, thereby confounding ATD neurochemical and behavioural results in rodents. As the ATD method seems important in the investigation of 5-HT-related vulnerability factors implicated in the onset of depression, the potential of alternative mechanisms and possible confounding factors should be taken into account for an adequate interpretation of data resulting from application of the method in both clinical and preclinical settings. In general, a degree of caution should be maintained in extrapolating the results of ATD studies to clinical depression. 


\section{Samenvatting}

De acute tryptofaan depletie (ATD) methode is momenteel de meest toegepaste challenge test in het onderzoek naar de betrokkenheid van serotonine (5-hydroxytryptamine, 5-HT) en 5-HT gerelateerde kwetsbaarheidsfactoren bij het ontstaan en het verloop van depressieve stoornissen. ATD is gebaseerd op de manipulatie van de beschikbaarheid van het essentiële aminozuur tryptofaan (TRP), de 5-HT precursor, door middel van het toedienen van een TRP-vrij dieet. Zowel het exacte onderliggende mechanisme van de neurofysiologische effecten van ATD als ook de mate waarin de veranderingen in de activiteit van 5-HT neuronen verantwoordelijk zijn voor de ATD-geïnduceerde neurochemische en gedragsveranderingen zijn niet volledig bekend. Dit belemmert een adequate interpretatie van de resultaten die verkregen worden na toepassing van de methode in humane en dierexperimentele experimenten. De studies beschreven in dit proefschrift hadden tot doel potentiële alternatieve onderliggende mechanismen van de neurofysiologische en gedragseffecten van ATD in ratten en muizen nader te onderzoeken door middel van het toedienen van een specifiek voedingsmengsel van eiwitten en koolhydraten op basis van gelatine.

Hoofdstuk 1 introduceert de biochemische principes die de basis vormen van de ATD methode door middel van een uiteenzetting van de specifieke elementen die kenmerkend zijn voor de biosynthese vanaf het essentiële aminozuur TRP in voedsel tot aan de modulerende 5-HT neurotransmitter in het brein. Zowel de algemene functies en eigenschappen van het 5-HT systeem als ook de specifieke veranderingen die een belangrijke rol spelen en nauw betrokken zijn bij de ontwikkeling van depressieve stoornissen worden uiteengezet. Er wordt een overzicht gegeven van de bruikbaarheid van de methode in depressieonderzoek met proefpersonen en preklinische resultaten die tot stand zijn gekomen na toepassing van de methode in ratten worden grondig besproken. Het basismechanisme dat ten grondslag ligt aan de inductie van perifere TRP depletie door middel van het toedienen van een TRP-vrij dieet wordt beschreven en de validiteit van de parameters die gebruikt worden voor de interpretatie van de biochemische effecten van ATD in het brein worden kritisch geëvalueerd. Vervolgens worden de doelstellingen van het proefschrift geïntroduceerd, namelijk het nader onderzoeken van potentiële alternatieve onderliggende mechanismen van de ATD methode en de capaciteit van de methode om affectieve en cognitieve veranderingen teweeg te brengen.

Hoofdstuk 2 geeft een overzicht van de meervoudige studies waarbij de farmacokinetische en gedragseffecten van diverse doseringsprotocollen van het TRP-vrije eiwit-koolhydraatmengsel onderzocht werden in muizen van het Swiss type en voornamelijk in C57BL/6 muizen. Gezien het feit dat latent aanwezige disfunctionele 5-HT systemen vandaag de dag extensief 
gemoduleerd worden in muizen, is het gebruik van muizen in plaats van ratten voor het bestuderen van gedrag in het algemeen en in het bijzonder de betrokkenheid van neurotransmittersystemen in psychiatrische stoornissen significant toegenomen. De initiële doelstelling was het opzetten van de ATD methode in de muis voor verdere potentiële toepassing als 5-HT challenge model in genetische muismodellen voor depressie. Perifere aminozuren werden gemeten en centrale TRP en 5-HT concentraties werden vergeleken met angsten depressie-gerelateerde gedrag in de elevated zero-maze (EZM), forced swim test (FST) en tail suspension test (TST). De mate van perifere TRP depletie was uiterst afhankelijk van de specificiteit van het doseringsvolume en het tijdsbestek van de meervoudige orale toedieningen. Terwijl ATD in Swiss muizen resulteerde in een afname van $74 \%$ van de ratio van TRP tot de som van andere grote aminozuren (TRP/ $/ \mathrm{LNAA})$ in plasma 1 uur na toediening $(2 \mathrm{x} 10 \mathrm{ml} / \mathrm{kg}, 30$ min interval), was in C57BL/6 muizen slechts een afname van 40\%. Deze muizen toonden ook geen angstgerelateerde gedrag in de EZM of een toename van immobiliteit in de FST of TST. Een hogere dosering $(2 \times 20 \mathrm{ml} / \mathrm{kg})$ met een langer interval (60 min) deed de ratio afnemen met 68\% in C57BL/ 6 muizen en verlaagde de 5-HT turnover in de hippocampus, maar had geen functionele effecten indien getest in de EZM en FST. Extreme verschillen in ATD effecten werden geobserveerd tussen de verschillende muizenstammen en geen enkele van de diepgaande perifere TRP depleties kwam overeen met centrale TRP of 5-HT veranderingen. Echter, interfererende stresseffecten gerelateerd aan de toepassing van de ATD methode leken de ATD-geïnduceerde effecten op de ratio TRP/ LLNAA in plasma te beïnvloeden. Deze bevindingen hebben belangrijke gevolgen voor het gebruik van ATD in het algemeen en in het bijzonder voor de toepasbaarheid in muizen. Het wordt verondersteld dat muizen met latent aanwezige 5-HT disfunctie mogelijk in hogere mate kwetsbaar zijn voor de effecten van ATD, hetgeen nader onderzocht zal moeten worden.

Hoofdstuk 3 beschrijft de effecten van ATD op de locale cerebrale doorbloeding (cerebral blood flow; CBF) en de mate van cerebraal glucose metabolisme (cerebral metabolic rate of glucose; CMRG). Deze werden gemeten in 43 verschillende hersenstructuren van volwassen mannelijke Wistar ratten aan de hand van $\left[{ }^{14 C} \mathrm{C}\right.$-iodoantipyrine en $\left[{ }^{14} \mathrm{C}\right]$-2-deoxyglucose kwantitatieve autoradiografische imaging. Ter vergroting van het inzicht in de potentiële onderliggende mechanismen van ATD-geïnduceerde neurochemische en gedragseffecten in het algemeen werden oriënterende experimenten voortgezet in het ratmodel van ATD. Gezien het feit dat de 5-HT neurotransmitter belangrijke vasoactieve eigenschappen bezit zou een acute afname in centrale 5HT concentraties veranderingen teweeg kunnen brengen in de CMRG en CBF en hun gewoonlijke onderlinge relatie. Dergelijke cerebrovasculaire veranderingen zouden een bijdrage kunnen leveren aan de onderliggende mechanismen van ATD-geïnduceerde veranderingen in affectief gedrag en 
cognitieve beperkingen. Hoewel de ratio TRP/ $\mathrm{LNNA}$ afnam met $40 \%$ werden er geen meetbare effecten gevonden van ATD op TRP en 5-HT concentraties in het brein. Desondanks reduceerde ATD de locale CBF aanzienlijk in 11 van de 43 onderzochte hersenstructuren, terwijl de locale CMRG onveranderd bleef. Bovendien werd een neerwaartse resetting van het verband tussen $\mathrm{CBF}$ en CMRG geobserveerd na ATD vergeleken met de TRP+ controlegroep, wijzend op een mate van cerebrovasculaire oligemie. De ATD-geïnduceerde afname in perifere TRP concentraties resulteerde dus in een verlaagde locale $\mathrm{CBF}$ en in een afwijking in het normale verband tussen de mate van doorbloeding al naar gelang de mate van metabolisme zonder veranderingen in centrale TRP en 5-HT concentraties. Derhalve wordt verondersteld dat een disfunctioneel 5-HT systeem eerder slechts een bijrol speelt in de onderliggende mechanismen van ATD.

Hoofdstuk 4 onthult de cerebrovasculaire effecten van ATD in een diermodel van verworven 5-HT disfunctie geïnduceerd door middel van herhaalde toedieningen met 3,4-methylenedioxymethamphetamine (MDMA; 'ecstasy'). Het is bekend dat de neurotoxiciteit van MDMA significante schade toebrengt aan 5-HT neuronen en een acute afname van 5-HT in het brein na ATD veroorzaakt mogelijk slechts affectieve gedragsveranderingen als er sprake is van een latent aanwezige kwetsbaarheid van het 5-HT systeem. Door middel van kwantitatieve autoradiografie imaging werd het effect gemeten van de herhaalde voorbehandeling met MDMA samen met een ATD challenge op de locale $\mathrm{CBF}$ en CMRG. Hierbij werd niet alleen de relatie tussen cerebrovasculaire functie en 5-HT veranderingen onderzocht, maar ook de MDMA-geïnduceerde 5-HT disfunctie als kwetsbaarheidfactor voor depressie in het algemeen en voor cognitive disfunctie in het bijzonder. MDMA induceerde significante 5-HT neurotoxiciteit en verlaagde centrale TRP en 5HT concentraties aanzienlijk. ATD resulteerde gemiddeld in een afname van $40 \%$ van de ratio TRP/ $\Sigma$ LNAA in plasma zonder de centrale TRP en 5-HT concentraties te veranderen. Een neerwaartse resetting van het verband tussen de mate van doorbloeding al naar gelang de mate van metabolisme werd alleen geobserveerd in de controlegroep die niet aan de drug blootgesteld was. Dit geeft aan dat ATD een significante opwaartse resetting van de relatie tussen $\mathrm{CBF}$ en CMRG veroorzaakt na voorbehandeling met MDMA vergeleken met de controlegroep waarbij het de MDMA-geïnduceerde cerebrovasculaire disfunctie lijkt te versterken. ATD produceert dus een zekere mate van cerebrovasculaire hyperperfusie in dieren die voorbehandeld zijn met MDMA. Dit zou mogelijk een kwetsbaarheidsfactor kunnen vormen voor een cerebrovasculair accident met in het bijzonder de kans op een hersenbloeding.

Hoofdstuk 5 toont de effecten van ATD op perifere en centrale concentraties van brain-derived neurotrophic factor (BDNF) in de rat rekening houdend met mogelijke stresseffecten van de specifieke experimentele procedures die de 
resultaten zouden kunnen beïnvloeden. De productie van BDNF hangt sterk samen met het 5-HT systeem en is niet alleen in extreme mate gevoelig voor stress, maar speelt ook een belangrijke rol in affectieve en geheugenprocessen en zou dus betrokken kunnen zijn bij het onderliggende mechanisme van ATDgeïnduceerde gedragsveranderingen. Een significante afname van 56\% van de ratio TRP/ $\mathrm{LLNAA}$ in plasma werd geobserveerd, echter, 5-HT metabolisme leek slechts minimaal aangetast te zijn in de hippocampus zonder meetbare veranderingen in centrale TRP of 5-HT concentraties. Hoewel geen directe effecten van ATD werden gevonden op de BDNF concentraties in serum of het brein werd een door stress veroorzaakte afname gevonden in BDNF concentraties in de prefrontale cortex. Bovendien correleerden centrale TRP concentraties positief met BDNF zowel in de prefrontale cortex als in de hippocampus. Veranderingen in BDNF concentraties als gevolg van ATD en/of de stress die gerelateerd is aan de toepassing ervan zou derhalve betrokken kunnen zijn bij het onderliggende mechanisme van ATDgeïnduceerde neurochemische en gedragsveranderingen.

Hoofdstuk 6 onderzoekt in hoeverre phosphodiesterase (PDE) remmers in staat zijn om een ATD-geïnduceerde verslechtering van de rat object herkenningsprestatie op te heffen. Hoewel affectieve gedragsveranderingen na ATD controversieel blijken worden ATD-geïnduceerde geheugendeficiënties consistent gerapporteerd in mensen, maar ook in ratten zoals gemeten met de rat object herkenningstest. Het remmen van PDE enzymen blijkt een betrouwbare methode voor het verbeteren van geheugenprocessen door middel van het verhogen van de concentraties van de second messenger moleculen gyclic adenosine monophosphate (cAMP) en cyclic guanosine monophosphate (cGMP) of beiden. Het nader onderzoeken van de pro-cognitieve effecten van PDE remmers kan meer inzicht geven in de potentiële interactie tussen second messenger moleculen en het 5-HT systeem als onderliggend mechanisme voor ATD-geïnduceerde cognitieve disfunctie. ATD verlaagde de ratio TRP/ $/$ LNAA in plasma en veroorzaakte een aanzienlijke verslechtering van de object herkenningsprestatie. Zowel de PDE5 remmer, vardenafil, als ook de PDE2 remmer, BAY 60-7550, die respectievelijk cGMP en zowel cAMP als cGMP verhogen, waren in staat om de object herkenningsprestatie na een ATD-geïnduceerde verslechtering acuut te verbeteren.

Hoofdstuk 7 geeft een samenvatting van de resultaten van de studies die beschreven worden in dit proefschrift en voorziet in een kritische discussie betreffende de potentiële alternatieve onderliggende mechanismen van de neurochemische en gedragseffecten van de ATD-methode. Over het algemeen wordt geconcludeerd dat de parameters die gebruikt worden om de afname van de beschikbaarheid van TRP in het brein en 5-HT metabolisme te berekenen niet uiterst nauwkeurig zijn. ATD-geïnduceerde gedragsveranderingen, als ook depressie zelf, zijn daarom niet gemakkelijk direct toe te schrijven aan 
veranderingen in 5-HT neurotransmissie. De bevindingen van dit proefschrift wijzen op de bijdrage van alternatieve mechanismen zoals een afname in de activiteit van nitric oxide synthase of cerebrovasculaire afwijkingen die betrokken zouden kunnen zijn bij de gedragseffecten van ATD en tevens 5-HTgemedieerde klinische depressieve symptomen zouden kunnen verklaren. Bovendien lijken de experimentele procedures die gerelateerd zijn aan de toepassing van de ATD-methode aanzienlijk stressvol en interfereren mogelijk met TRP metabolisme, hetgeen de pure neurochemische en gedragseffecten van ATD in knaagdieren mogelijk in de war stuurt. Gezien het feit dat de ATDmethode belangrijk is voor onderzoek naar 5-HT-gerelateerde kwetsbaarheidsfactoren die betrokken zijn bij het ontstaan van depressie zou er rekening gehouden moeten worden met de potentie van alternatieve mechanismen en mogelijke interfererende factoren voor een zorgvuldige interpretatie van data die voortkomt uit de toepassing van de methode in zowel humane als dierexperimentele studies. In het algemeen lijkt het raadzaam om voorzichtig om te gaan met het direct extrapoleren van de resultaten van ATD studies naar de klinische symptomen van depressieve stoornissen. 



\section{Resumen}

La técnica de la depleción aguda de triptófano (ATD por sus siglas en inglés) representa hoy en día la prueba de desafío más utilizada para investigar la implicación de la serotonina (5-HT), el sistema serotonérgico así como factores de vulnerabilidad relacionados en la patogénesis y la fisiopatología de la depresión. La ATD consiste en la manipulación del triptófano (TRP), un amino ácido esencial que es precursor de la 5-HT, a través de una dieta especial carente del mismo. El mecanismo exacto detrás de los efectos neurofisiológicos, y el nivel en el que alteraciones en la actividad neuronal de 5HT contribuyen a modificaciones neuroquímicas y cambios en el comportamiento inducido por ATD no se conocen del todo. Los estudios descritos en la presente tesis tuvieron como objetivo explorar mecanismos alternativos mediante los cuales ATD ejerce sus efectos neurofisiológicos y de comportamiento.

El capítulo 1 introduce la idea general del uso de la técnica de ATD. Describe las características específicas de la biosíntesis del TRP, desde la adquisición del mismo a través de la dieta hasta la síntesis de 5-HT, un neurotransmisor modulador en el cerebro. Explica las funciones y características generales del sistema serotonérgico, así como su implicación en la depresión mayor. Presenta un panorama general de la utilidad de la técnica de ATD en la investigación de la depresión en humanos y se revisan de manera profunda los datos derivados de la aplicación de la técnica. Muestra el mecanismo básico de la depleción del TRP periférico mediante la administración de una dieta carente del mismo y se evalúa críticamente la validez de los parámetros que se suelen utilizar para la interpretación de los efectos bioquímicos de la depleción de TRP en el cerebro. A continuación se introducen los objetivos de la tesis que consisten en la exploración de posibles mecanismos alternativos subyacentes a la técnica de ATD y su potencial para inducir cambios de comportamiento tanto afectivos como cognitivos.

El capítulo 2 presenta un resumen de los múltiples estudios, en los cuales se investigaron los efectos farmacocinéticos y conductuales de diferentes dosis del compuesto proteico y de hidratos de carbono sin TRP, en ratones tipo Swiss y particularmente en ratones tipo C57BL/6. Hoy en día el sistema serotonergico disfuncional es modulado de manera extensa en ratones, por lo que el uso de ratones en vez de ratas para estudiar el comportamiento en general y específicamente la implicación de sistemas de neurotransmisores en trastornos psiquiátricos, ha incrementado significativamente. El objetivo inicial se basaba en el ajuste de la técnica de ATD en el ratón con la posible aplicación de la técnica como prueba de desafió de la 5-HT en ratones transgénicos, modelos animales de depresión. Los amino ácidos fueron medidos periféricamente y los niveles centrales de TRP y de 5-HT fueron comparados con el grado de 
conducta de ansiedad y depresiva en el laberinto elevado en cero (EZM), la prueba de nado forzado (FST) y el test de suspensión en la cola (TST). El grado de depleción de TRP periférico dependía en gran medida de la especificidad del volumen de la dosis y del tiempo entre las múltiples administraciones. Mientras que en los ratones del tipo Swiss ATD provocó una reducción del 74\% de la proporción de TRP comparado con la suma de los otros amino ácidos largos (TRP/ $/$ LNAA) una hora después a la administración $(2 \times 10 \mathrm{ml} / \mathrm{kg} ; 30$ minutos de intervalo), en los ratones del tipo C57BL/6 se efectuó una reducción de sólo un 40\%. Estos últimos no mostraron ansiedad en el EZM o un incremento en la inmovilidad en el FST o TST. Una dosis más alta $(2 \times 20 \mathrm{ml} / \mathrm{kg})$ con un intervalo más largo (60 minutos) redujo la proporción un 68\% en los ratones del tipo C57BL/6 y disminuyó el recambio de 5-HT en el hipocampo, pero no causo efectos conductuales en el EZM o FST. Se observaron grandes diferencias entre los distintos tipos de ratones y las profundas depleciones de TRP, las cuales en ningún momento correspondían con cambios en el TRP o 5HT cerebral. Sin embargo, se encontró que el estrés relacionado a la aplicación del ATD interfiere con los efectos inducidos por ATD respecto a la proporción TRP/ $/$ LNAA en el plasma. Estos resultados tienen una implicación importante para el uso de ATD en general y particularmente para su aplicación en ratones. Probablemente, ratones con una disfunción de 5-HT pre-existente podrían ser más vulnerables al ATD.

El capítulo 3 describe los efectos de la ATD sobre el flujo sanguíneo cerebral $(\mathrm{CBF})$ y la tasa metabólica de glucosa cerebral (CMRG). Ambos parámetros fueron medidos en 43 áreas distintas del cerebro de ratas adultas del tipo Wistar. Para esto fue utilizada la técnica de imagenología auto radiográfica cuantitativa de [14C]-iodoantipirina y [14C]-2-desoxiglucosa, respectivamente. Para ampliar la comprensión de los posibles mecanismos responsables de los efectos neuroquímicos y conductuales de la ATD se continuó con los experimentos de exploración en el modelo establecido de ATD en la rata. El neurotransmisor 5-HT posee importantes características vasoactivas, un decremento agudo de los niveles de 5-HT podría alterar el CMRG y el CBF y la relación que existe entre ambos bajo condiciones fisiológicas normales. Este tipo de alteraciones cerebrovasculares podrían contribuir con los mecanismos responsables de los cambios conductuales de tipo afectivo y con el déficit cognitivo. Mientras que la proporción TRP/ $\mathrm{LLNAA}$ disminuyo un 40\%, no se pudo observar un efecto perceptible inducido por la ATD sobre los niveles de TRP y el de 5-HT en el cerebro. A pesar de ello, la ATD redujo el CBF local significativamente en 11 de las 43 áreas de interés, mientras que la CMRG se mantuvo similar. Además, se pudo observar que el ATD produjo que la relación entre el CBF y la CMRG fue decreciente en comparación con el grupo control, TRP+, indicativo de un estado aparente de oligoemia cerebrovascular. Así, el decremento de los niveles periféricos de TRP inducido por la ATD resulto en una disminución del CBF local y un desacoplamiento del flujo y 
metabolismo sin afectar los niveles cerebrales de TRP o 5-HT. Por eso se sugiere que un sistema disfuncional de la 5-HT podría ser un mecanismo complementario a la ATD.

El capítulo 4 revela los efectos cerebrovasculares de la ATD en un modelo animal de disfunción serotonérgica adquirida, inducida por la administración repetitiva de 3, 4-metilenedioximetanfetamina (MDMA; 'extasis'). Se sabe que la neurotoxicidad de la MDMA causa una disminución significativa de las terminales neuronales serotonergicas y un decremento agudo de los niveles de 5-HT. Después de la ATD probablemente exista un trastorno en el comportamiento afectivo solamente si se presenta de antemano una vulnerabilidad del sistema de 5-HT. Con el propósito de investigar la relación entre la función cerebrovascular, los cambios en el sistema serotonérgico y la disfunción del sistema 5-HT inducido por el MDMA como factor de vulnerabilidad para la depresión en general así como para la disfunción cognitiva en particular, se midió el efecto del pre-tratamiento con MDMA juntos con la ATD sobre el CBF y el CMRG local. La MDMA provoco una neurotoxicidad significativa del sistema 5-HT y disminuyo los niveles cerebrales de TRP y de 5-HT considerablemente. ATD resulto en una reducción de un $40 \%$ de la proporción TRP/ $2 \mathrm{LNAA}$ plasmática sin afectar los niveles cerebrales de TRP o 5-HT. Se observó que solamente el tratamiento con ATD en los animales del grupo control (sin el tratamiento farmacológico) hizo que decreciera la relación entre CBF y CMRG. Esto indica que, en comparación con el grupo control, la ATD per se provoco que esta relación aumentara después del pre-tratamiento con MDMA potenciando así la disfunción cerebrovascular inducida por el MDMA. Así pues, la ATD produce un grado de hiperperfusión cerebrovascular en animales pre-tratados con MDMA lo cual podría indicar una predisposición a los accidentes cerebrovasculares, particularmente al derrame cerebral.

El capítulo 5 muestra los efectos de la ATD sobre el factor neurotrófico derivado del cerebro (brain-derived neurotrophic factor, BDNF), controlando las posibles situaciones que en la rata provocan estrés y que posteriormente influyen en el análisis de los resultados obtenidos. El BDNF esta fuertemente relacionado con el sistema de 5-HT y no sólo es muy sensible al estrés, si no que también tiene un papel esencial en la regulación del ánimo y el procesamiento de la memoria, por lo cual podría contribuir con el mecanismo que provoca las alteraciones conductuales inducidas por la ATD. Se encontró una disminución significativa de un $56 \%$ de la proporción TRP/ 2 LNAA plasmática, no obstante, el metabolismo de 5-HT aparentemente sólo se afectó de manera moderada en el hipocampo. Aunque no se encontraron efectos directos sobre los niveles de BDNF en el suero o en el cerebro, se detectó un decremento en los niveles de BDNF en la corteza frontal producido por el estrés. Además, se pudo observar una correlación positiva entre los niveles 
cerebrales de TRP y los de BDNF tanto en la corteza frontal como en el hipocampo. Las alteraciones en los niveles de BDNF causado por la ATD o el estrés relacionado con su aplicación podrían entonces ser los responsables de las alteraciones neuroquímicas y conductuales inducidas por la ATD.

El capítulo 6 evalúa la capacidad de los inhibidores de la fosfodiesterasa (phosphodiesterase; PDE) para revertir el déficit en la ejecución de la tarea de reconocimiento de objetos inducido por la ATD. Aunque las alteraciones del comportamiento afectivo inducidas por la ATD permanecen controvertibles, el déficit de la memoria inducida por la ATD se observa consistentemente tanto en humanos como en ratas cuando se mide mediante el test de reconocimiento de objetos. La inhibición de las fosfodiasterasas es un método confiable para mejorar los procesos de memoria mediante un incremento de los niveles de los segundos mensajeros: adenosínmonofosfato cíclico (AMPc) y guanosín monofosfato cíclico (CMPc) o ambos. El análisis de los efectos pro-cognitivos de inhibidores de PDE podría mejorar la comprensión de la posible interacción entre moléculas del tipo segundo mensajero y el sistema 5-HT como mecanismo de la disfunción cognitiva inducida por la ATD. La ATD resultó en un decremento significativo de la proporción plasmática de TRP/ 2 LNAA y afectó la tarea de reconocimiento de objetos considerablemente. Tanto el inhibidor de PDE5, vardenafil, y el inhibidor de PDE2, BAY 60-7550, que incrementan GMPc y CMPc-AMPc respectivamente tuvieron la capacidad de mejorar la ejecución de la memoria a corto plazo después de un déficit inducido por la ATD.

El capítulo 7 resume los resultados de los estudios descritos en esta tesis y ofrece una discusión crítica con respecto a posibles mecanismos alternativos de los efectos neuroquímicos y conductuales de la técnica de la ATD. En general se puede concluir que los parámetros utilizados para calcular la disminución de la disponibilidad de TRP y el decremento en el metabolismo de 5-HT son poco precisos. Por todo esto resulta difícil asociar la depresión, así como las alteraciones conductuales inducidas por la ATD directamente con cambios en la neurotransmisión de 5-HT. Los resultados de esta tesis apoyan la contribución de mecanismos alternativos tales como el decremento en la actividad de la enzima oxido nítrico sintasa y las anormalidades cerebrovasculares con los efectos conductuales observados después de la ATD y podrían explicar también la sintomatología clínica de depresión mediada por 5-HT. Los procedimientos relacionados con la aplicación de la técnica de ATD resultan muy estresantes per se y podrían interferir con el metabolismo de TRP confundiendo así los resultados neuroquímicos y comportamentales de la ATD en roedores. La técnica de ATD parece ser importante para la investigación de los factores de vulnerabilidad relacionados al sistema de la 5-HT implicados con el establecimiento de la depresión. Se debe tener en cuenta la importancia de los mecanismos alternativos y los posibles factores de confusión para una 
interpretación adecuada de los datos obtenidos con la aplicación de la técnica en un marco tanto clínico como preclínico. En general, debe tener cierto cuidado con la extrapolarización de resultados obtenidos utilizando la técnica de ATD a la depresión clínica. 



\section{Acknowledgements}

I challenged my serotonin system en kwam boven...thanks to all of you!

Special thanks go out to my promotor and copromoters. I'm more than grateful for the oportunity you gave me to grow both professionally and personally. You have made it all possible in the first place!

Prof. Steinbusch, Harry, bedankt voor alle deuren die voor mij open konden, van het begin tot het eind en door weer en wind. Newmood was een challenge, bedankt dat ik die aan mocht gaan!

Dr. Prickaerts, Jos, soms wilde ik net iets sneller dan jij en jij net iets anders dan ik en zie hier het resultaat! Bedankt voor je inmense inzet en volste vertrouwen, meten is weten, dat hebben we samen ondervonden!

Dr. Kelly, dear Dr. Paul, my time in Edinburgh has changed my life forever! What a fabulous experience and in such a professional environment, where you gave me the opportunity to learn the ins and outs of cerebrovascular research. You created a new home for me and have been so very much personally involved, from the very beginning until the very end. I will keep improving my Scottish, I can't say it enough...ta!

Dr. Blokland, Arjan, ook al was het afscheid al eens daar, ik kon toch nog niet zonder je. Bedankt voor je inzet en waardevolle adviezen. Jij had het voor ogen, het einddoel is bereikt!

Dr. Ferrington, Linda, making a "kitty" (quintessentially Dutch) is how we first met and in Edinburgh I found in you a wonderful new colleague and dearest friend. We have been like the codes of our samples, LE! Together we made the blood flow...'the neurosurgeons' of The Spiegeltent! You introduced me in your life and culture and have made me feel so at ease and at home always, especially at the 5 star "FerrEtch" hotel. You and Colin are the best hosts ever! Everything has been very much to my likening! It was an honour to be able to dance with Colin at the caleigh of your wedding. I now hope you feel as honoured to be my paranymph!

Marjo, met jou heb ik alles kunnen delen, jouw luisterend oor is goud waard! Samen spinnen of mokken en een cappuchino 'op z'n tijd' en niet te vergeten, de planning van onze katers! Wat kunnen ze het leuk vinden samen...Zonder de steun en toeverlaat van jou en Casper was het allemaal lang zo dragelijk niet geweest. Je bent een waardevolle vriendin en ik kan me geen betere paranimf bedenken!

Dr. Dawson, Neil, you have been a wonderful colleague, always willing to help and show me around, not only in the Edinbrrrra lab, but also in the pub for an after-work pint! Thanks for all your time and dedication. Your contribution to my amazing time in Edinburgh is unforgettable! 
Special thanks also to Dr. Henry Olverman, Darren and Craig and to all the other helpful people from the University of Edinburgh.

Mijn in-vivo collega's en kamergenoten Olga Reneerkens, Jochen De Vry, Sven Akkerman en Eva Bollen, bedankt voor alle gezelligheid, op, na en buiten het werk, ver weg op congres of dichtbij in de stad. Ook voor jullie geduld en begrip tijdens mijn wat wat mindere 'in-vivo' periodes ben ik jullie erg dankbaar. Ik wens jullie allemaal een snelle en succesvolle afronding toe en stay in-vivo, het is om trots op te zijn, dat is ons vaak genoeg gezegd! In het bijzonder Daniël van den Hove en Kris Rutten, jullie waren mijn grote voorbeeld. Bedankt voor al jullie hulp en adviezen en de onvoorwaardelijke steun!

Also to all the other room-mates and Neuroscience colleagues, Paul, Leonidas, Marijke, Annerieke, Eveline, Rinske, Thibeault and all the others, thanks for the nice moments, fruitful discussions and enlightning coffee breaks! Mi querida Marisela, tal y como has estado en estos momentos conmigo, estaré contigo cuando sea necesario. Que trabajito te he dejado para última hora. Mil gracias! Jodi, it's good fun with you and thanks for your approval! Former colleagues, Matteo, grazie, and Pawel Driekuje! Cindy Lieben en Linda Jans, jullie proefschriften waren mijn 'bijbel', bedankt voor alle adviezen! Ayhan Ş1k, Iyi tatiller, you were the best! Laura Smits, niet meer dichtbij, maar nog altijd betrokken, bedankt voor al je hulp! Marie-Thérèse en Anouk, bedankt voor alle administratieve hulp en zorgzaamheid! Anja, we biked the sites in Washington D.C., it was amaaaazing! Nicole, bedankt voor alle goede EURON zorgen! Marianne Markerink, zonder jou was ik nooit van picogram naar kilogram gekomen. Bedankt voor al je uitleg en geduld! En Wiel Honig, wat is een labtop zonder jou?! Ook Nadine en Allard en alle andere medewerkers van het CPV, bedankt voor jullie hulp en de prettige samenwerking. In het bijzonder Richard Frijnts, naast collega, nu ook vecino! En niet te vergeten, de meiden van de DE Uns 50, jullie hebben me wakker gehouden. Bedankt voor de heerlijke capucchino's met liefde gemaakt en...zonder cacao!

Beste Wil, mijn zelfvertouwen heeft tijdelijk in jouw handen gelegen. Bedankt voor je positive kijk en je motiverende woorden. Ik beloof het te zullen vieren!

Onmogelijk was het geweest zonder de steun en toeverlaat van mijn Mestreechse vrun en vrienden in Maastricht!

Rob, altijd een luisterend oor, menig probleem heb ik met jou kunnen delen, toen nog bij El Mercado en nu nog altijd! Bedankt voor alle diepe gesprekken, je aandacht en steun!

Karin, wat kunnen we heerlijk klagen en samen de wereld analyseren, maar vooral onszelf. Bedankt voor al je hulp, steun en de gezellige momenten. Je bent een super meid en mega vriendin! LSD rules! 
Cristina, que alegría tenerte como amiga. Gracias por tu apoyo e interés, no me puedo imaginar tener que celebrar esto sin tí. Te voy a echar mucho de menos!

Antonio, muchas gracias por quedarte aquella noche y por ser parte del principio de mi vida actual!

Guillermo Schreier, me salvaste la vida en su momento, virtualmente! Gracias por el escucharme y por simplemente estar allí!! Y por la ñ!

Femke en Stefan, jullie waren me voor en daar heb ik veel aan gehad! Bedankt voor jullie Belgisch-Limburgse gezelligheid, adviezen en steun!

Nina Davids, bedankt voor al je steun en hulp! You are next! Have fun in the USA, ik mis je...

Menig donkere bui is door een tuna uitje verlicht! Muchas gracias a todas, Fortunitas! Yanti, jij was als laatste nog dichtbij, cuánto admiro tu espíritu tunero y que bien nos lo pasamos! Aúpa Tuna! Y él que no diga olé...!

Hoe dichter bij Dordt, hoe...verder weg van Maastricht, en toch zijn jullie altijd zo dichtbij gebleven. Jochem, Esther, Daan, Elske, Feddo, Debbie, Geert, Nander, Sjon en alle anderen Dordtse vriendjes en vriendinnetje, bedankt voor alle mooie en bijzondere momenten, jullie onvoorwaardelijke steun en de eeuwige warme onthalen!

Silke, ya podemos ir de viaje, Las Reinas de España! He podido sacar tanta energía de los recuerdos tán bonitos y 'granainos' que tenemos en común. Gracias por seguir reviviéndolos conmigo!

Fernando, los primeros años estabas tú alli, a mi lado y luchando conmigo. Gracias por tu apoyo y por los buenos momentos juntos. Say hi to Edinburgh for me!

Dani, mil gracias por tu tiempo y dedicación. No te puedes imaginar lo que significa esto para mí. Que artistas sois, d2d rules! Un beso también pá Lalu y Lola!

Ook Chawwa en Marianne en Johan de Zoete, bedankt voor al jullie hulp en adviezen!

Amigos de La Bodega, gracias por hacerme sentir como en casa para así poder desconectar en condiciones! Extra picos is alleen voor V.I.P's!

A mis padrinos Cuqui y Perico con los que me hubiera gustado compartir este momento. Os echo de menos...

Niet te vergeten al mijn harige vriendjes, klein en groot, wit en zwart. Uiteindelijk de enige echte helden van dit project! De wetenschap en ik zijn jullie eeuwig dankbaar. Had ik maar nog meer kunnen vervangen, verminderen en verfijnen... 
Jaime, mijn allerliefste broer, que grande eres y que arte tienes. Alles wat ik niet heb, heb jij en andersom, somos como el yin y el yan, un complemento puro. Que más se puede pedir entre hermanos! Agradezco tu plena confianza, je onvoorwaardelijke liefde y más que nada, tu paciencia! Bedankt voor je inmense inzet voor de afronding van dit boekje. Ik bewonder je y te quiero mucho!

Lieve mama, dit is wat jij hebt gebreid, helemaal alleen. Estoy tán orgullosa de tí! Je onvoorwaardelijke liefde is inmens en heeft er toe geleid dat ik nu ben wie ik ben, net als jij, groot en sterk. He podido compartirlo todo contigo y siempre has estado a mi lado....te quiero con toda mi corazón en ik ben je eeuwig dankbaar voor al je inzet, hulp en mentale steun als beste moeder van de wereld en vriendin voor het leven. You were right... alles sal reg kom...

Roses, la mitad es tuyo, eres un verdadero Dr. de la Vida, mi coach personal...El Mejor! Te debo la vida, a tí y a Sufi, el mayor experto en ratones! Todo esto no hubiera sido posible sin tu inmenso amor y apoyo constante. Juntos lo hicimos y juntos llegamos a la meta, nos pondrán el mismo tiempo...RvD pá siempre, Andalucía nos querrá! Eres too good to be true y lo mas bonito que me ha pasado en la vida...te quiero...cipote!

¿Que silbe el viento!

Todo lo que empieza, se acaba... 


\section{Curriculum vitae}

Eva van Donkelaar was born on July $5^{\text {th }} 1978$ in Jerez de la Frontera, Spain, where she enjoyed sunny life until she sputtered her first Spanish words. She then moved to the Netherlands where she completed her secondary education in 1997 at Thuredrecht College in Dordrecht. In 1998 she relocated to Maastricht to study Biological Psychology in the Faculty of Psychology and Neuroscience at Maastricht University. The Socrates free-movers program gave her the opportunity to spend the academic year 2000-2001 in the Faculty of Psychology at the University of Granada in Spain which allowed her to go back to her roots and get familiar with Spanish university life. To finalize her Master's degree in Neuropsychology she stayed in Spain, but moved further south to Cádiz where she took her first steps into the scientific world. After more than a year of extensive research training in preclinical psychopharmacology in the Neuropsychopharmacology Department within the Faculty of Medicine of the University of Cádiz, she returned to Maastricht in 2004 to graduate as a biological psychologist. Following her graduation she took a temporary post working as a behavioural pharmacology research assistant in the Department of Neuroscience within the faculty of Medicine, Health and Life Sciences at Maastricht University where she started as a $\mathrm{PhD}$ student in 2005. In 2006 she was awarded a Mari-Curie training grant which gave her the opportunity to visit the Cerebrovascular Research Laboratory at the University of Edinburgh in Scotland where she enjoyed 10 months working on aspects of her research project as it is described in this thesis. 



\section{Publications}

\section{Journal articles}

van Donkelaar EL, van den Hove DLA, Blokland A, Steinbusch HWM, Prickaerts J (in press). Stress-mediated decreases in central brain-derived neurotrophic factor as potential confounding factor for acute tryptophan depletion induced neurochemical effects. Eur Neuropsychopharmacol.

Rutten K, van Donkelaar EL, Ferrington L, Blokland A, Bollen E, Steinbusch HWM, Kelly PAT, Prickaerts J (2009). Phosphodiesterase inhibitors enhance object memory independent of cerebral blood flow and glucose utilization in rats. Neuropsychopharmacology, Mar 4, [Epub ahead of print].

van Donkelaar EL, Rutten K, Akkerman S, Blokland A, Steinbusch HWM, Prickaerts J (2008). Phosphodiesterase 2 and 5 inhibition attenuates the object memory deficit induced by acute tryptophan depletion. Eur J Pharmacol 600(1-3): 98-104.

van Donkelaar EL, Ferrington L, Blokland A, Steinbusch HWM, Prickaerts J and Kelly, PAT (in press). Acute tryptophan depletion in rats alters the relationship between cerebral blood flow and glucose metabolism independent of central serotonin. Neuroscience.

van Donkelaar EL, Kelly PAT, Dawson N, Blokland, A, Prickaerts J, Steinbusch HWM and Ferrington L (submitted). Altered cerebrovascular control following acute tryptophan depletion in MDMA-pretreated rats. J Neurosci Res.

van Donkelaar EL, Blokland A, Lieben, CKJ., Kenis G, Ferrington L, Kelly PAT, Steinbusch HWM, Prickaerts J (submitted). Acute tryptophan depletion in healthy C57BL/6 mice does not induce reduction of central serotonin levels or affective behavioural changes. Neurochem Int.

\section{Abstracts}

van Donkelaar EL, Kelly PAT, Dawson N, Blokland A, Prickaerts J, Steinbusch HWM and Ferrington L (2008/2009). MDMA-induced serotonergic dysfunction, a vulnerability factor for depression, predisposes rats to cerebrovascular abnormalities after acute tryptophan depletion. Society of Neuroscience Meeting 2008 (poster presentation), Washington DC (USA) and 8th Endo-Neuro-Psycho Meeting 2009 (poster presentation), Doorwerth (NL).

van Donkelaar EL, Blokland A, Kenis G, Ş1k A, Ferrington L, Kelly PAT, Steinbusch HWM, Prickaerts J (2008). Pharmacokinetic and behavioural effects of acute tryptophan depletion in mice. European Behavioural Pharmacology Society International Workshop on Behavioural Genetics 
and Neuropsychiatric Disorders (poster presentation), Cork (Ireland). Published in: Behav Pharmacol 19, 663-663.

van Donkelaar EL, Kelly PAT, Prickaerts J, Steinbusch HWM, Ferrington L (2007). Acute tryptophan depletion in rats produces uncoupling of flow from metabolism which parallels human depression. Institute of Psychiatry Meeting, Royal Society of Medicine. Depression: Brain Causes, Body Consequences (poster presentation), London (UK).

van Donkelaar EL, Steinbusch HWM, Lieben CKJ, Kenis G, Blokland A, Deutz NEP, Prickaerts J (2006). Pharmacokinetics of acute tryptophan depletion in male Swiss mice. Edinburgh Neuroscience Day (poster presentation), Edinburgh (UK) and 5th Endo-Neuro-Psycho Meeting (poster presentation), Doorwerth (NL).

van Donkelaar EL, Steinbusch HWM, Van Dalen J, van den Hove DLA, Kenis G, Blokland A, Deutz NEP, Prickaerts J (2006). Pharmacokinetics of acute tryptophan depletion in male Swiss mice. Edinburgh Neuroscience Day (poster presentation), Edinburgh (UK) and 5th Dutch Endo-NeuroPsycho Meeting (poster presentation), Doorwerth (NL).

van Donkelaar EL, Blokland A, Jans L, Kenis G, Steinbusch HWM, Prickaerts J (2005). The effects of acute tryptophan depletion on BDNF protein levels in brain and serum of male and female rats. 4th Endo-NeuroPsycho Meeting (poster presentation), Doorwerth (NL) and 11th biennial meeting of the European Behavioural Pharmacology Society (poster presentation), Barcelona (SP). Published in: Behav Pharmacol 16, S89-S89.

van Donkelaar EL (2006). Acute tryptophan depletion in the mouse: Challenging the serotonergic system. 5th Endo-Neuro-Psycho Meeting (oral presentation), Doorwerth (NL). 